S21

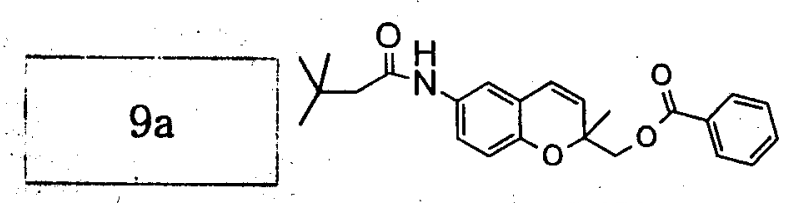

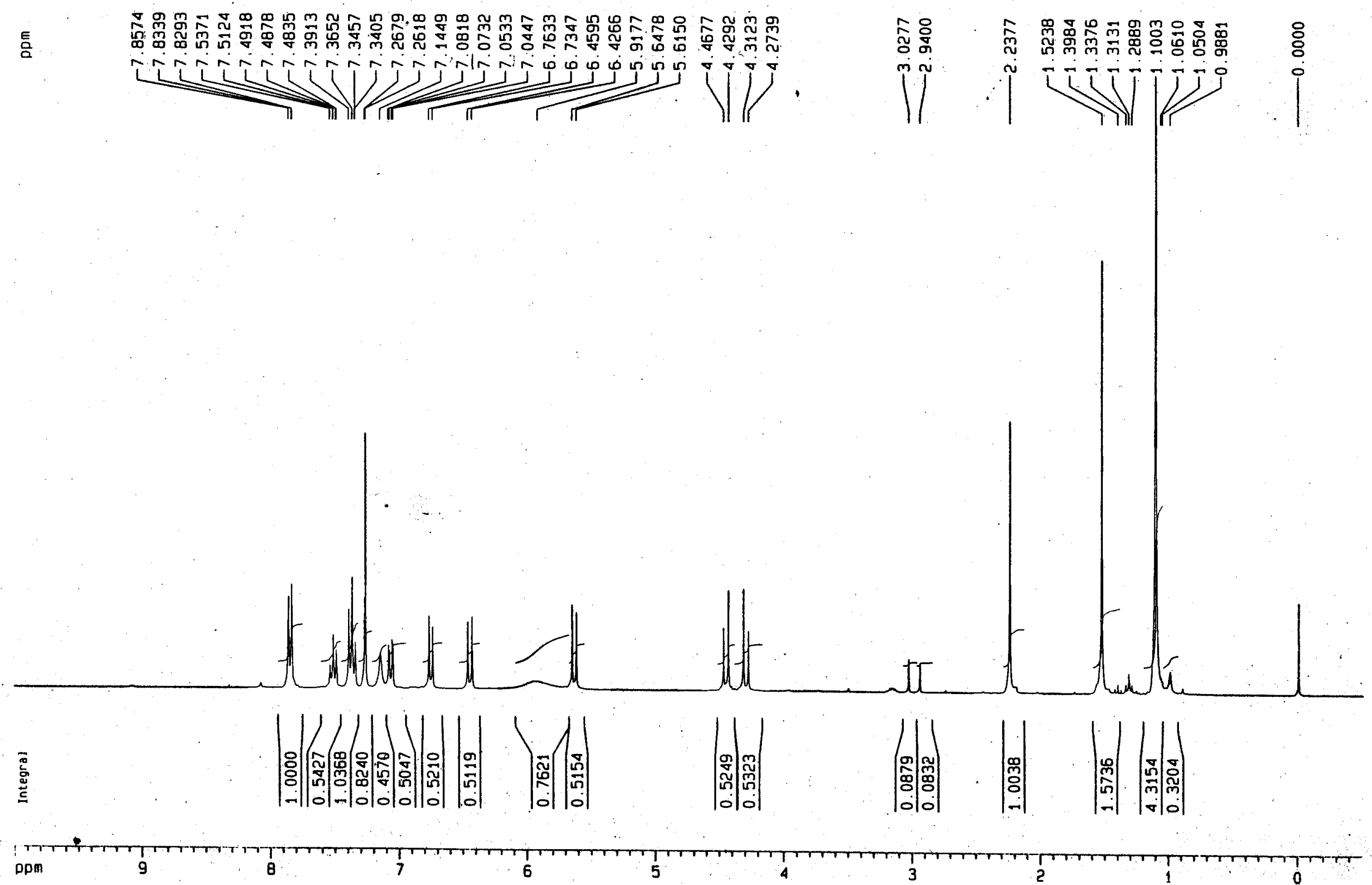




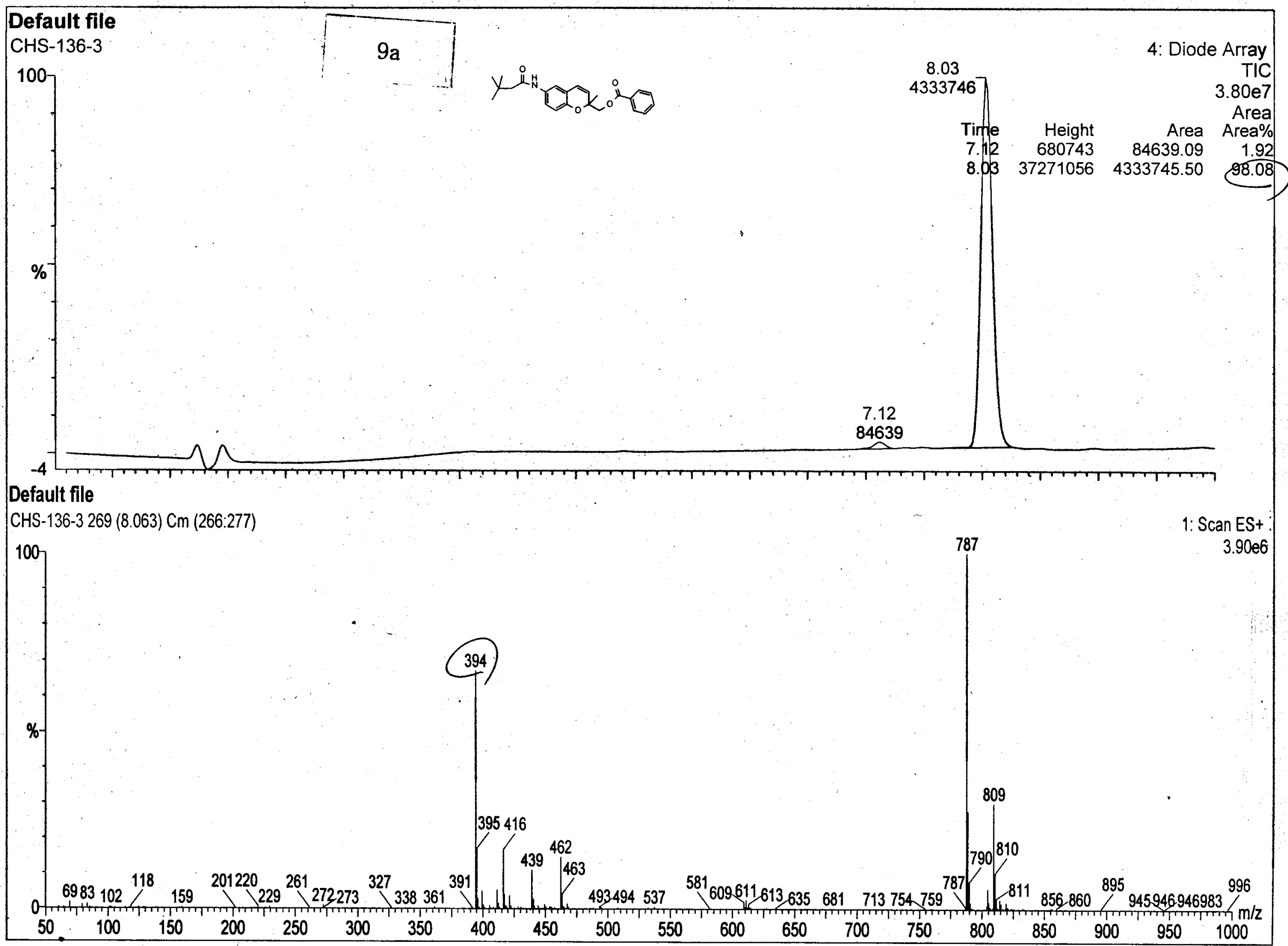




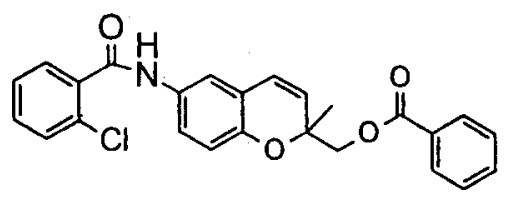

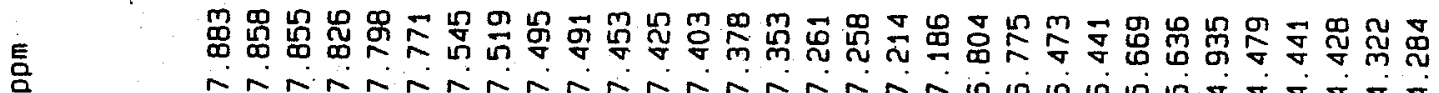

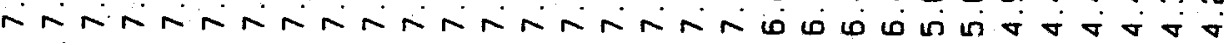
$\longrightarrow$
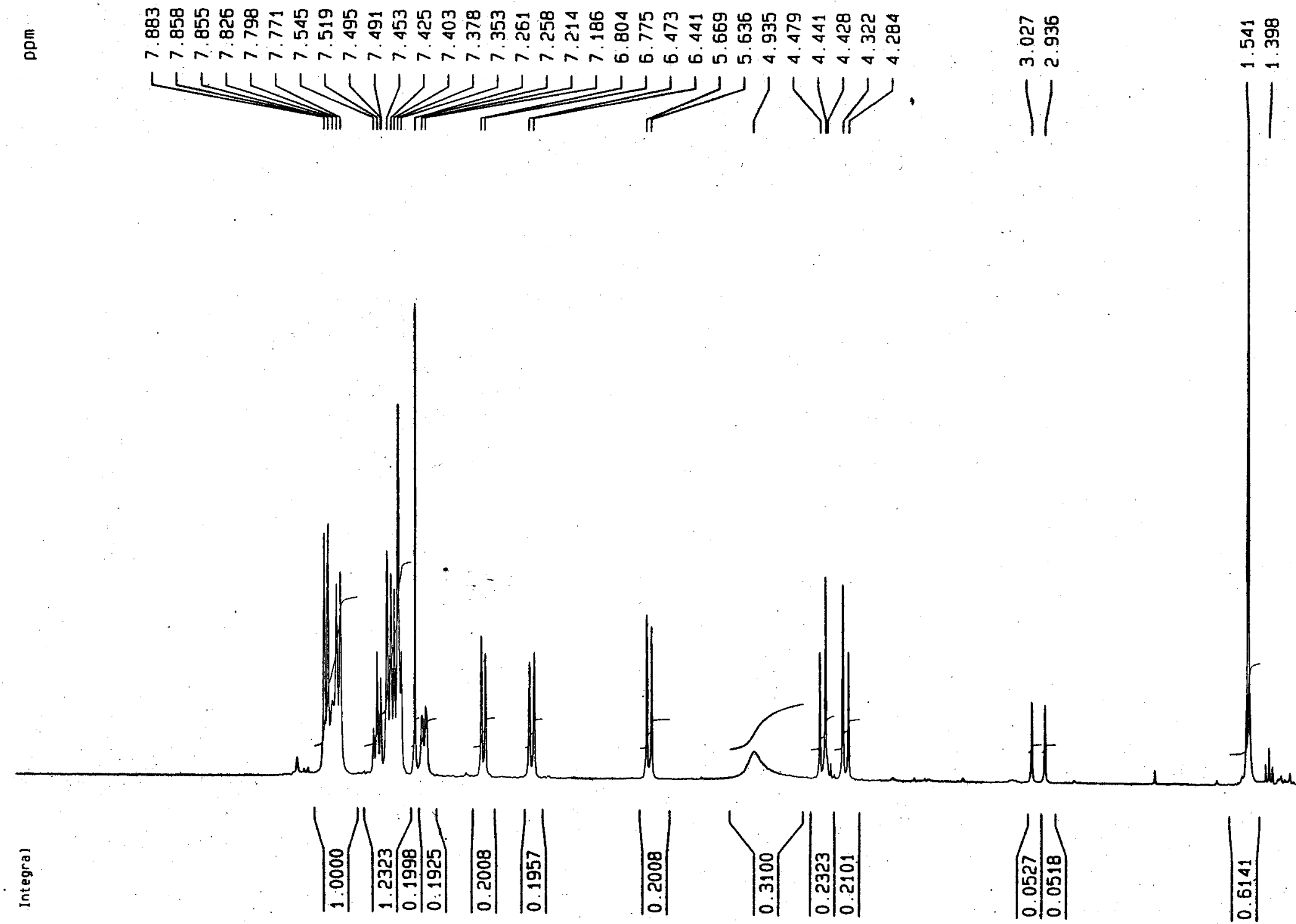

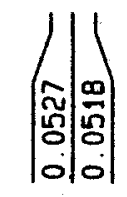

$\left|\begin{array}{c}\mid \\ : \\ 0 \\ 0\end{array}\right|$ 


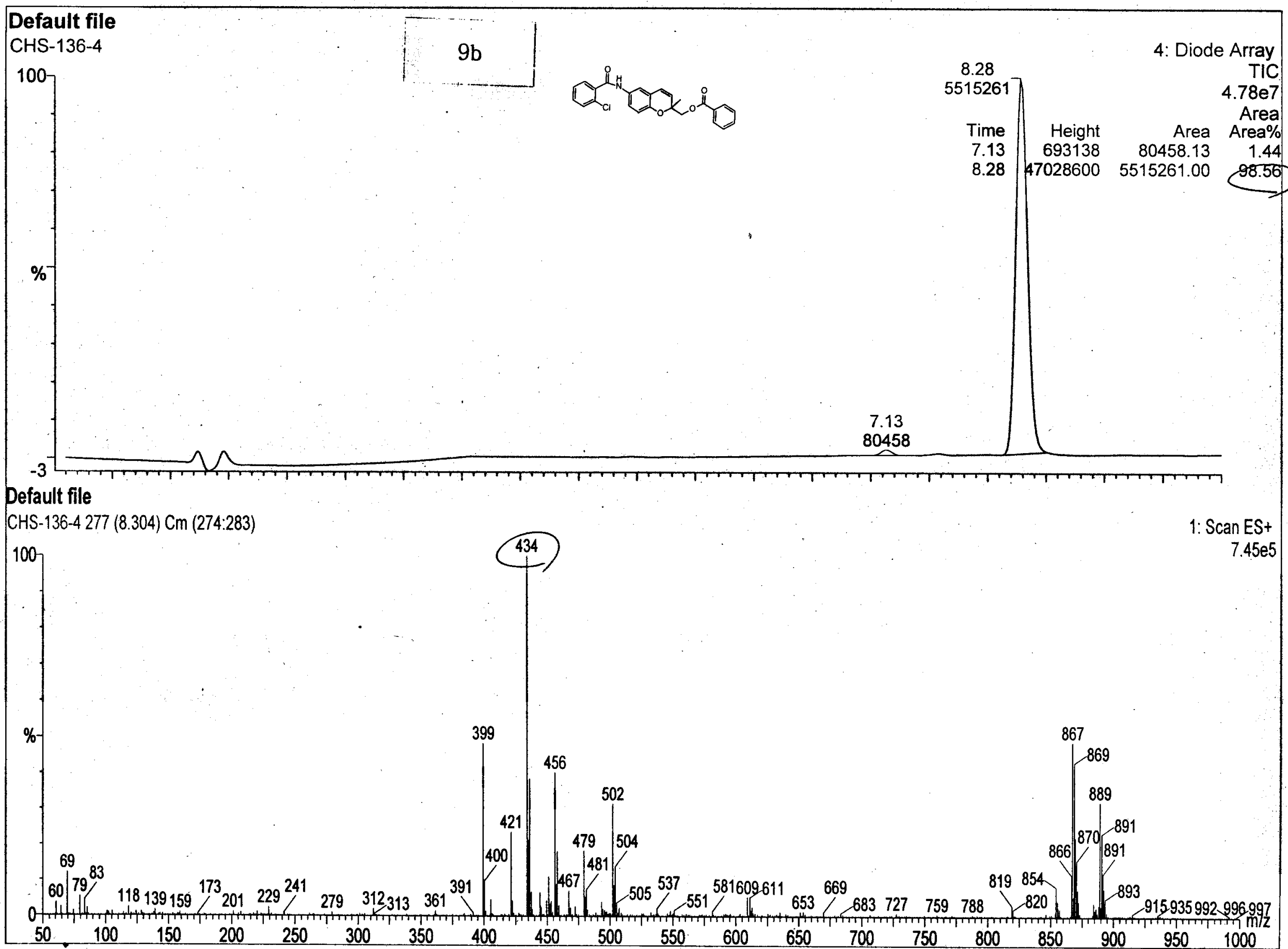


S25

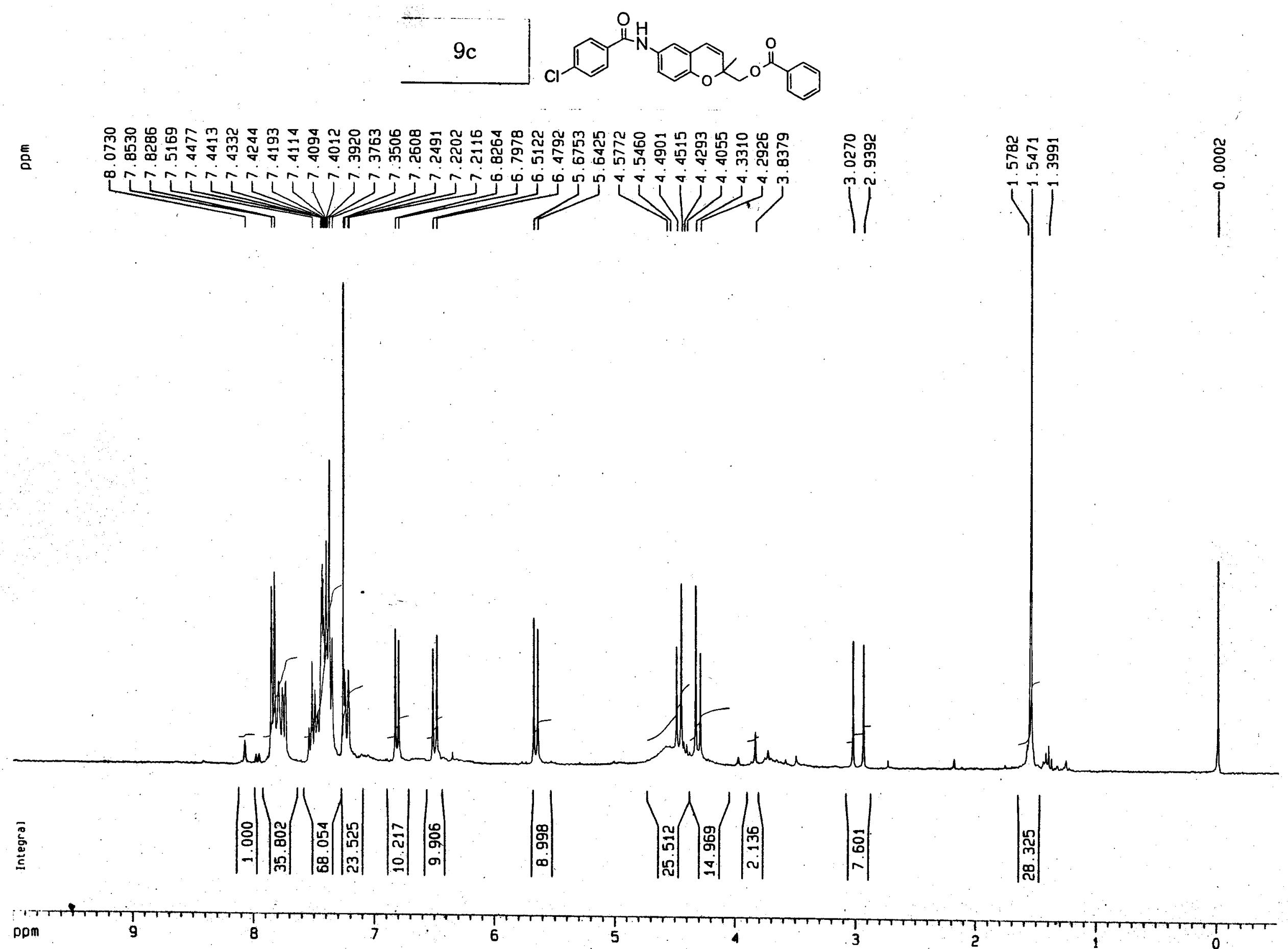




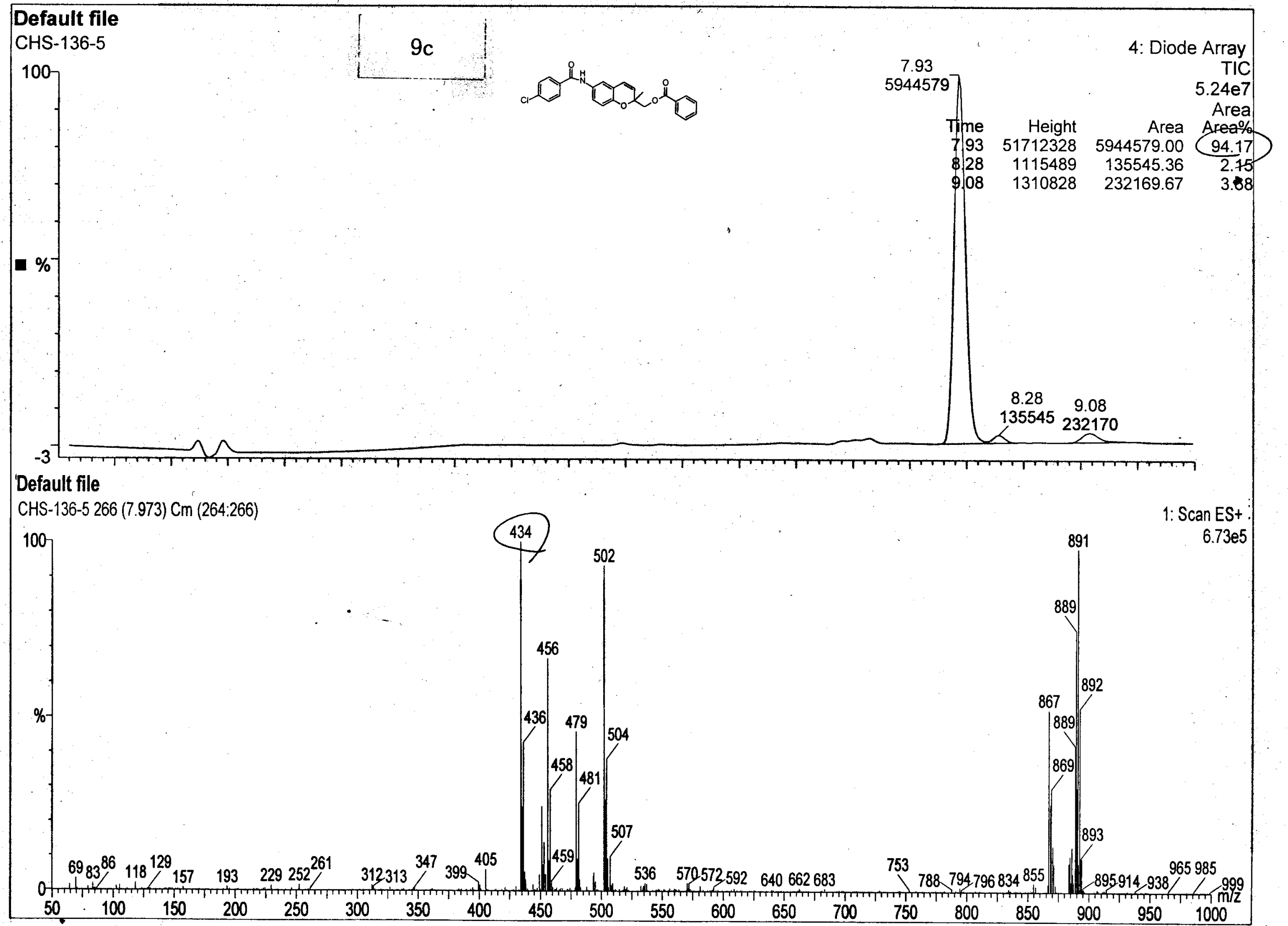


S27

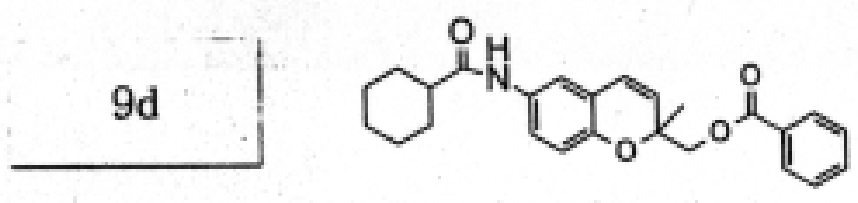

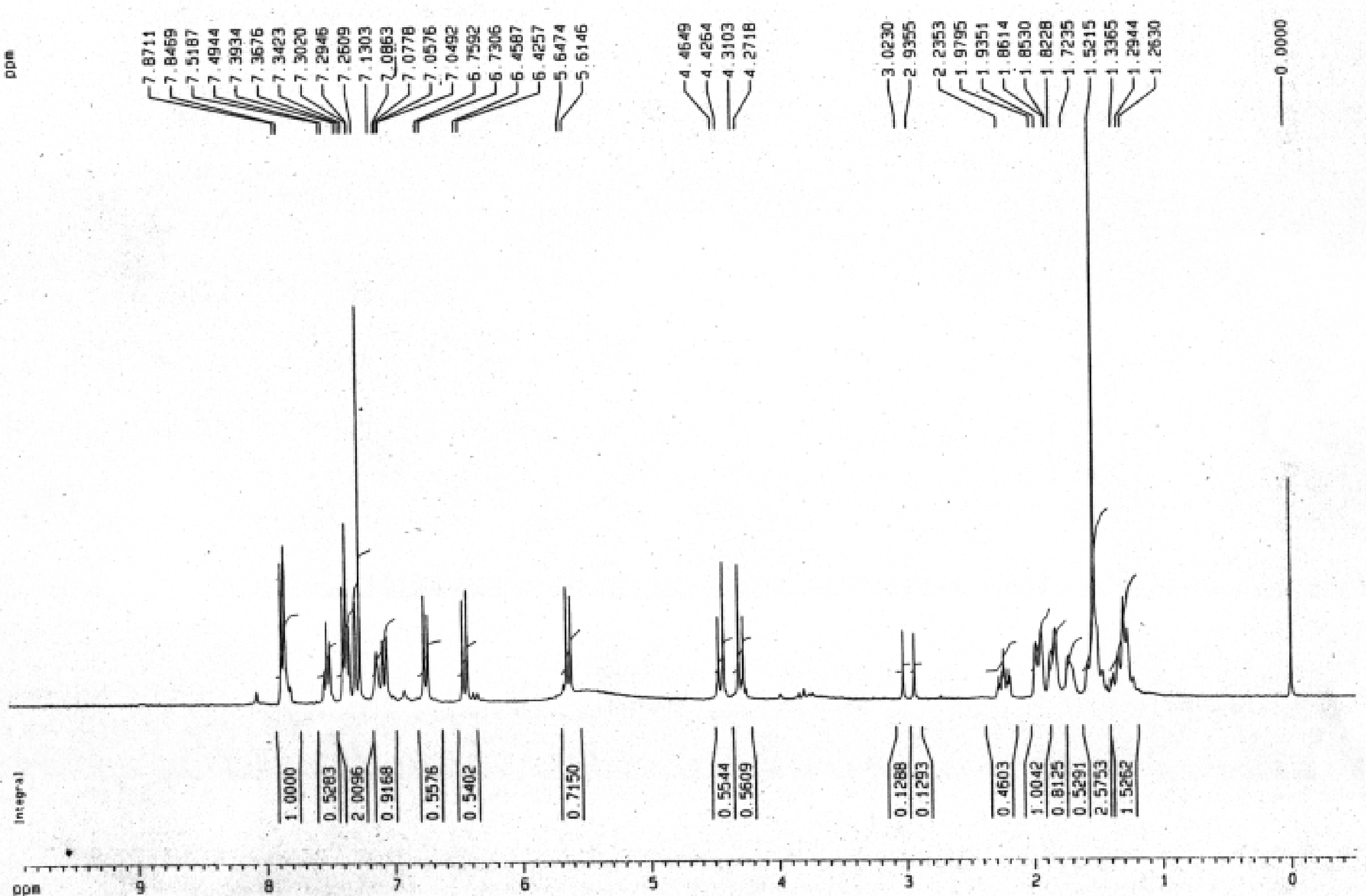




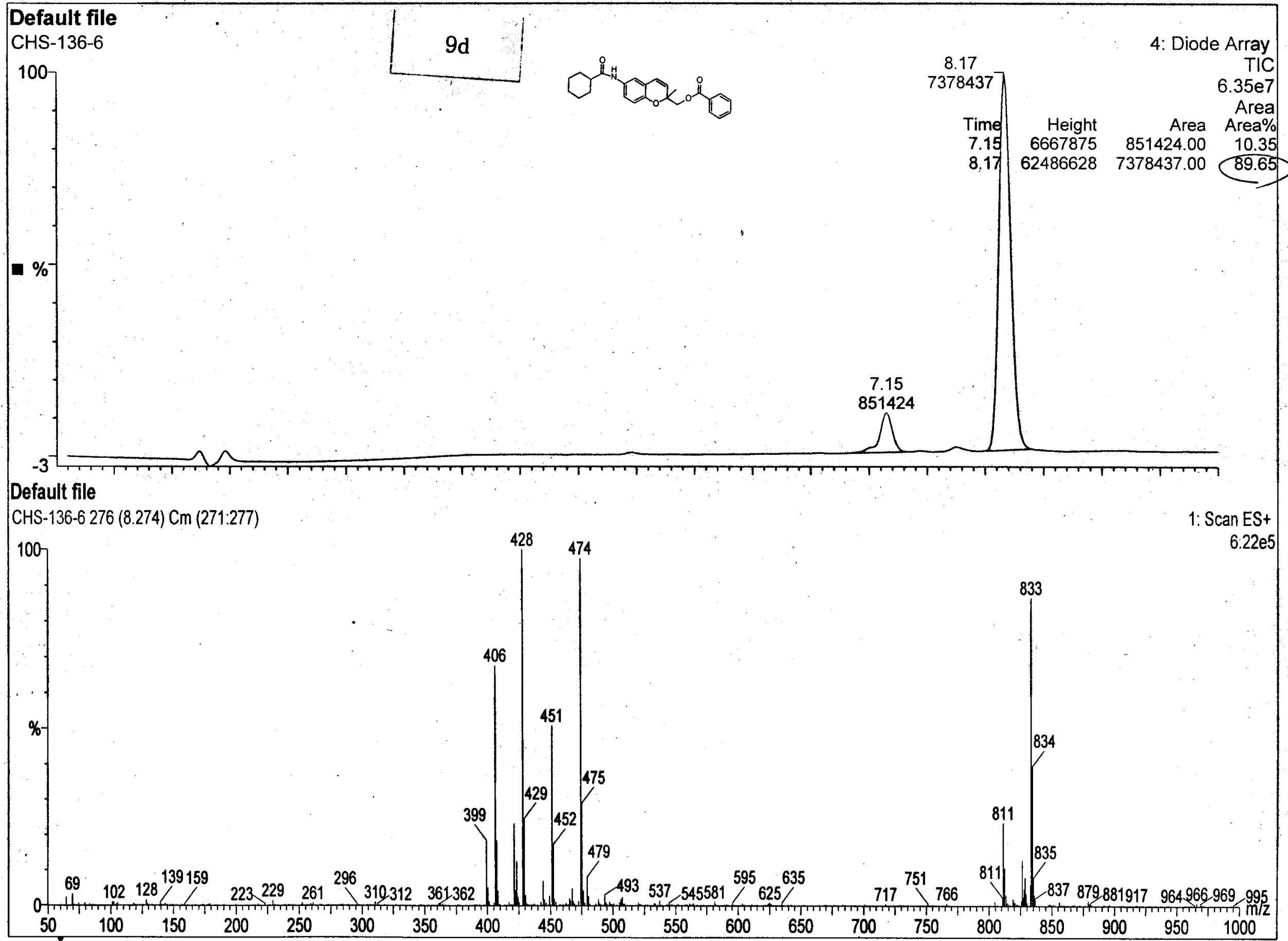




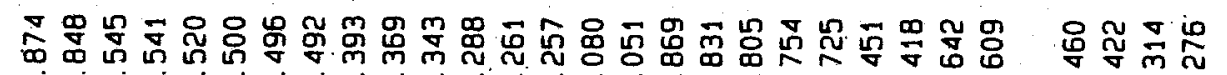
rarrararararión $\overbrace{}^{11}$

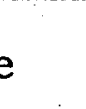

वं
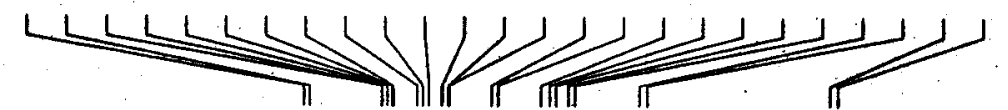

111
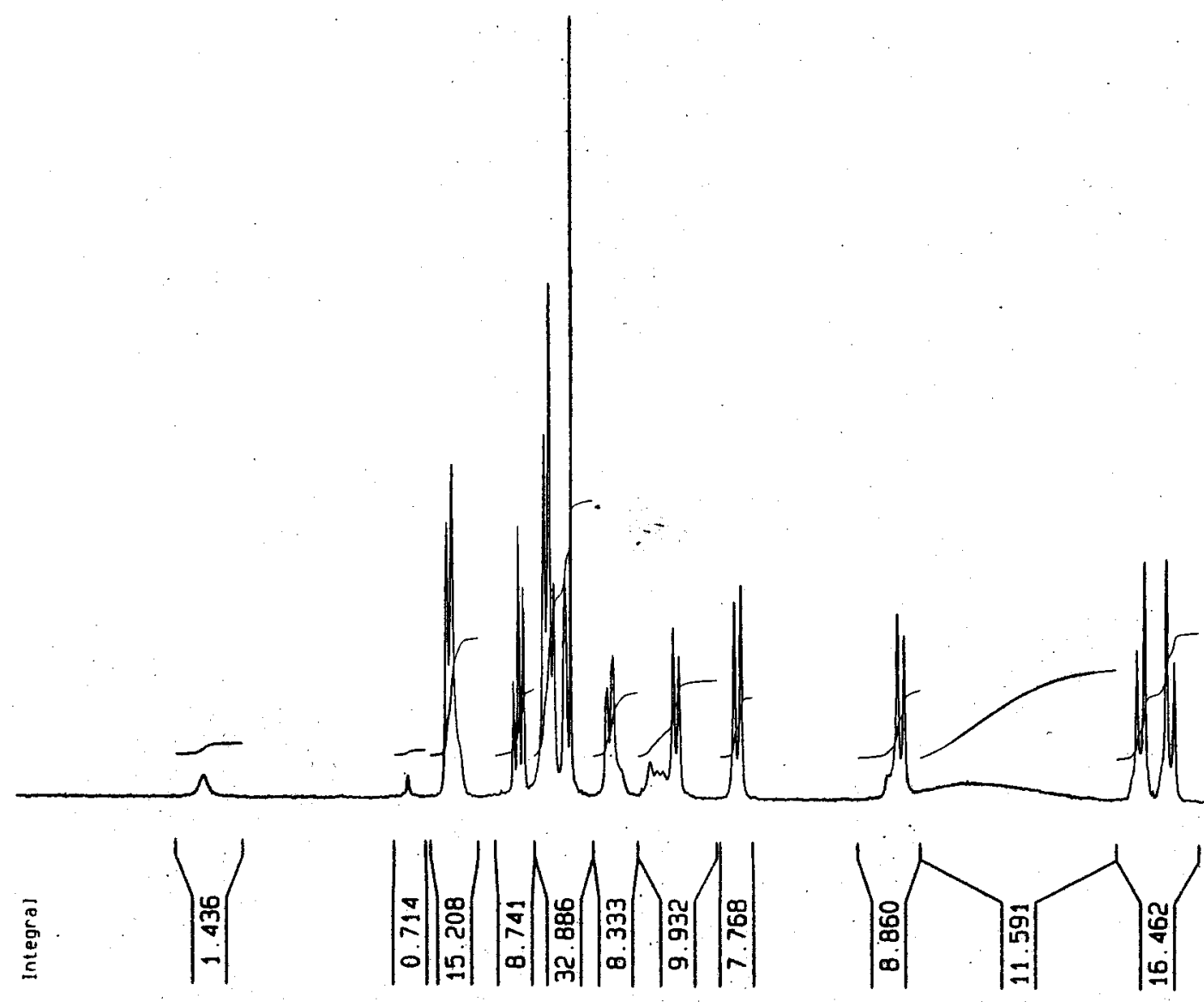

$\frac{\infty}{\sigma} \underset{m}{o n}$
$\mid$
min

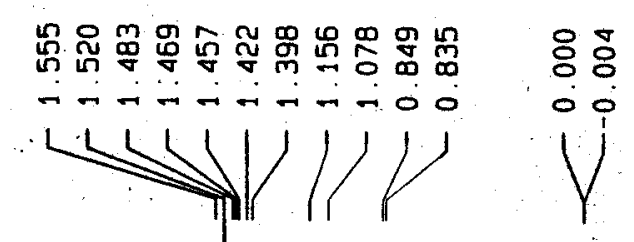

8.

1

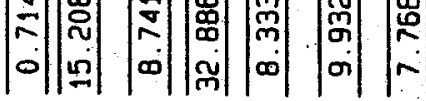

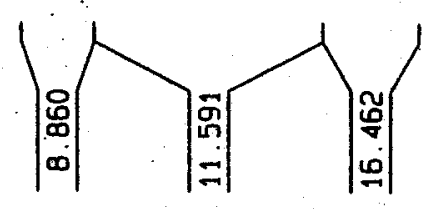

Ht

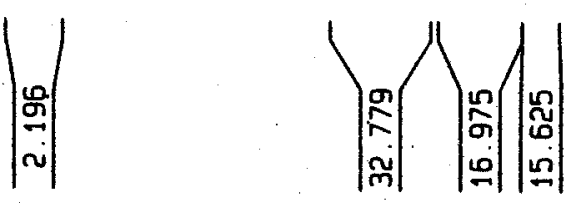




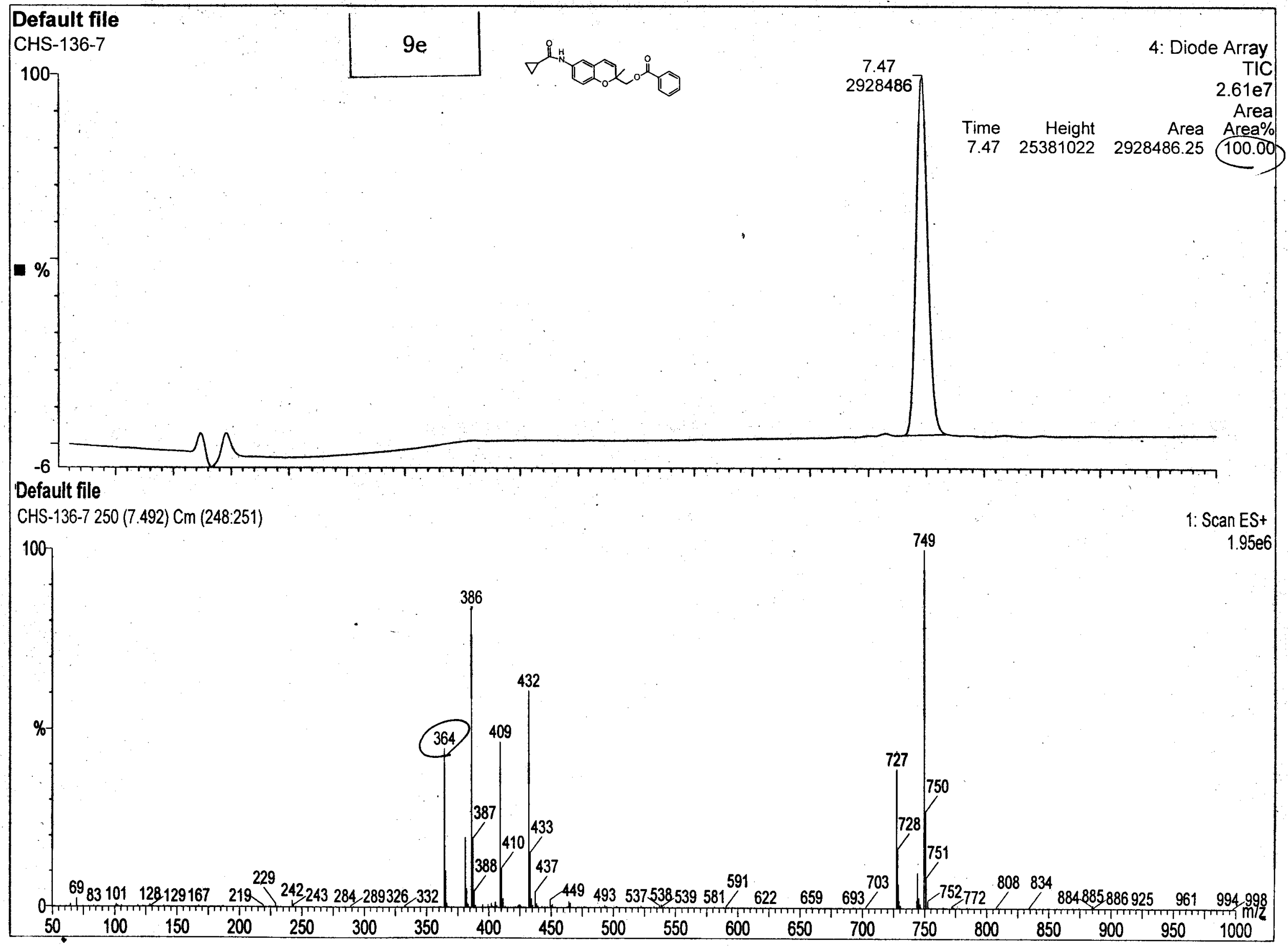



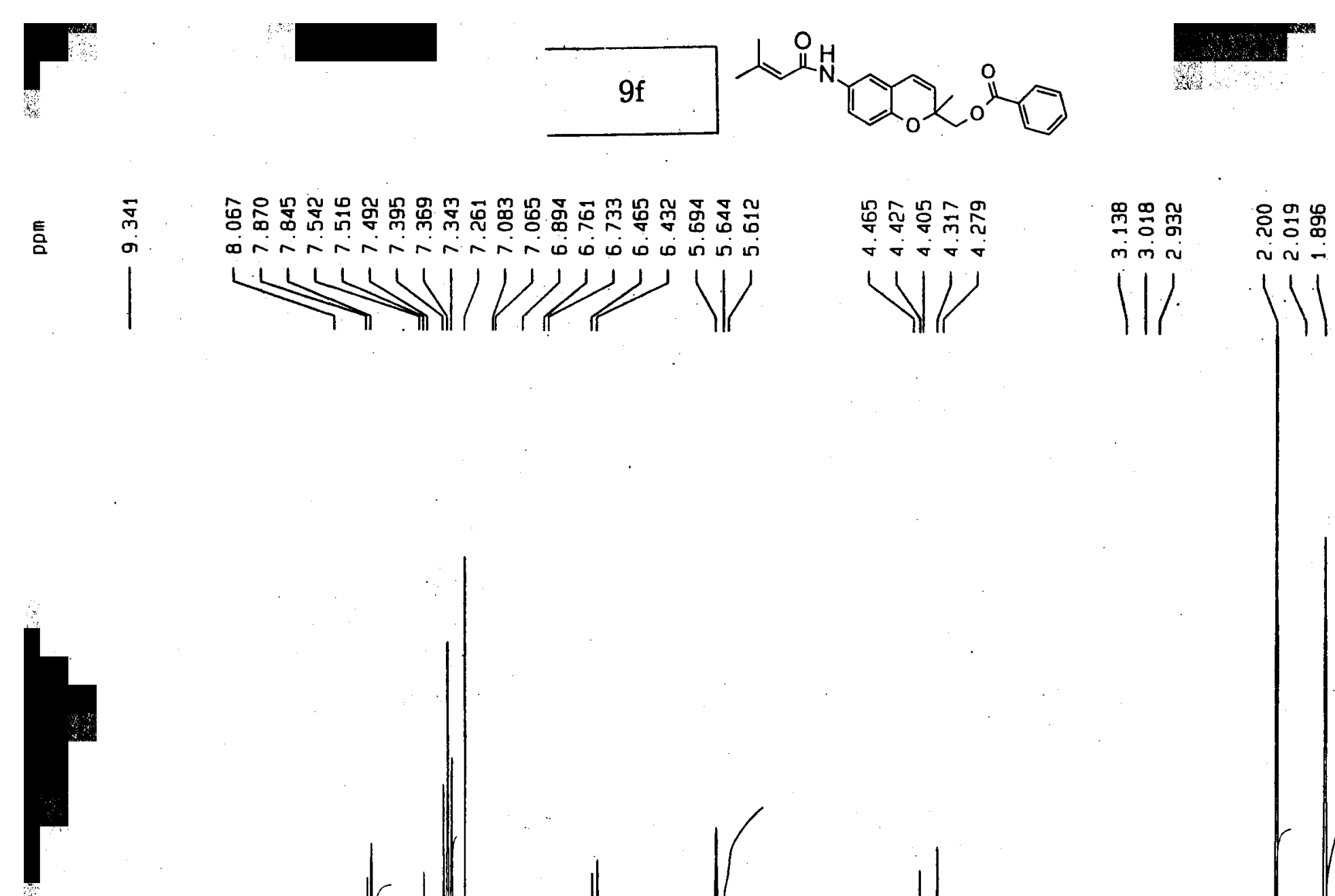

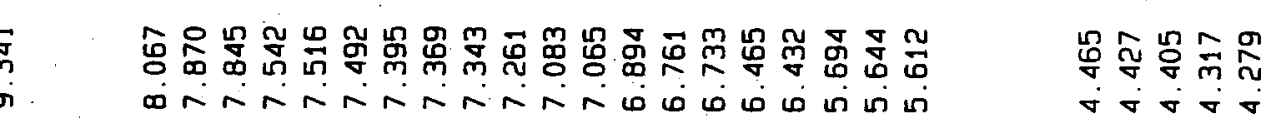

MI

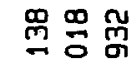

लिं का

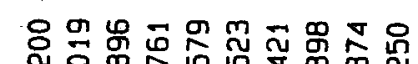

W

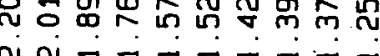

$\int 1111111$

$11 \%$

i
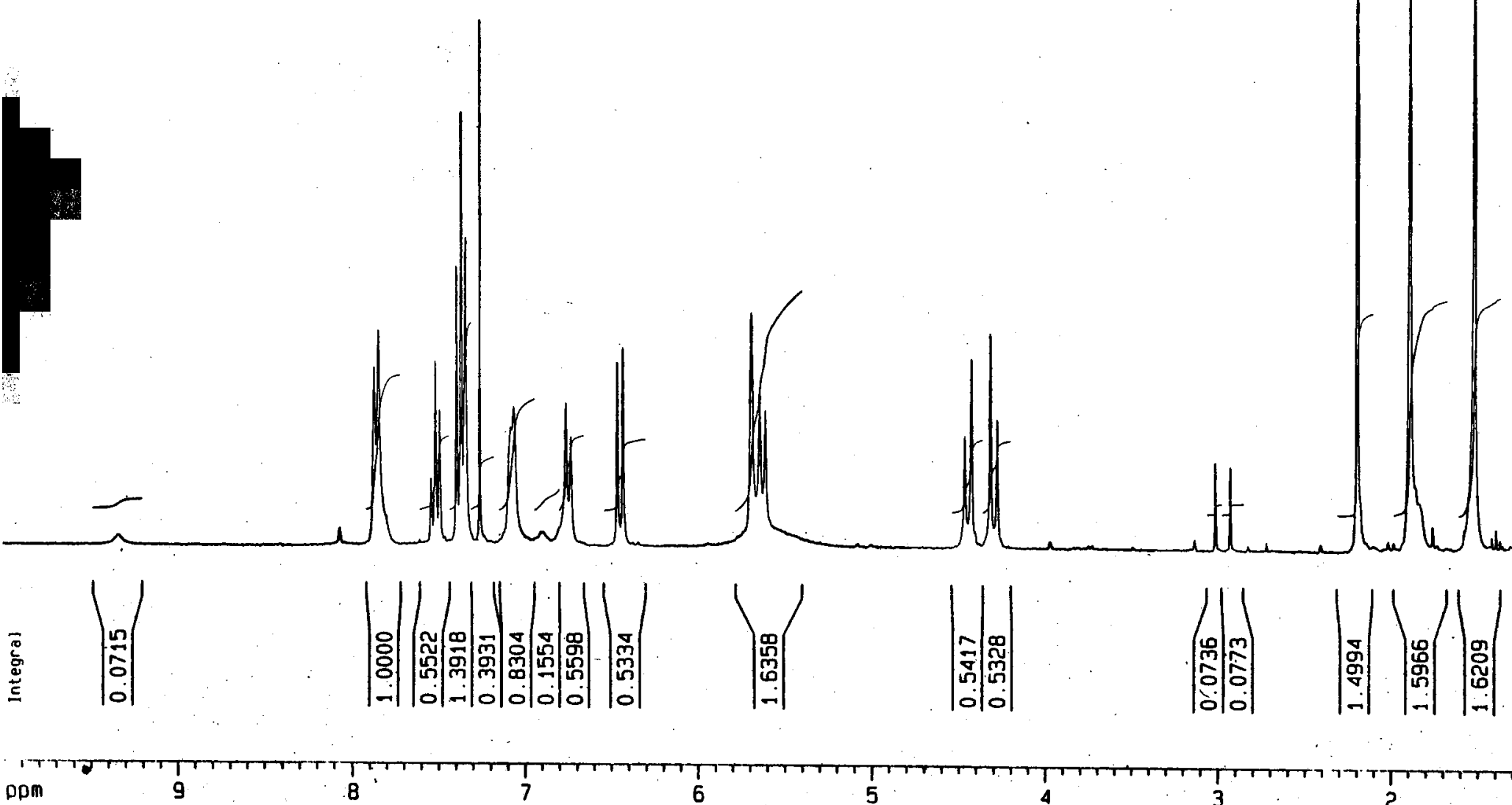

9

8

7

6 


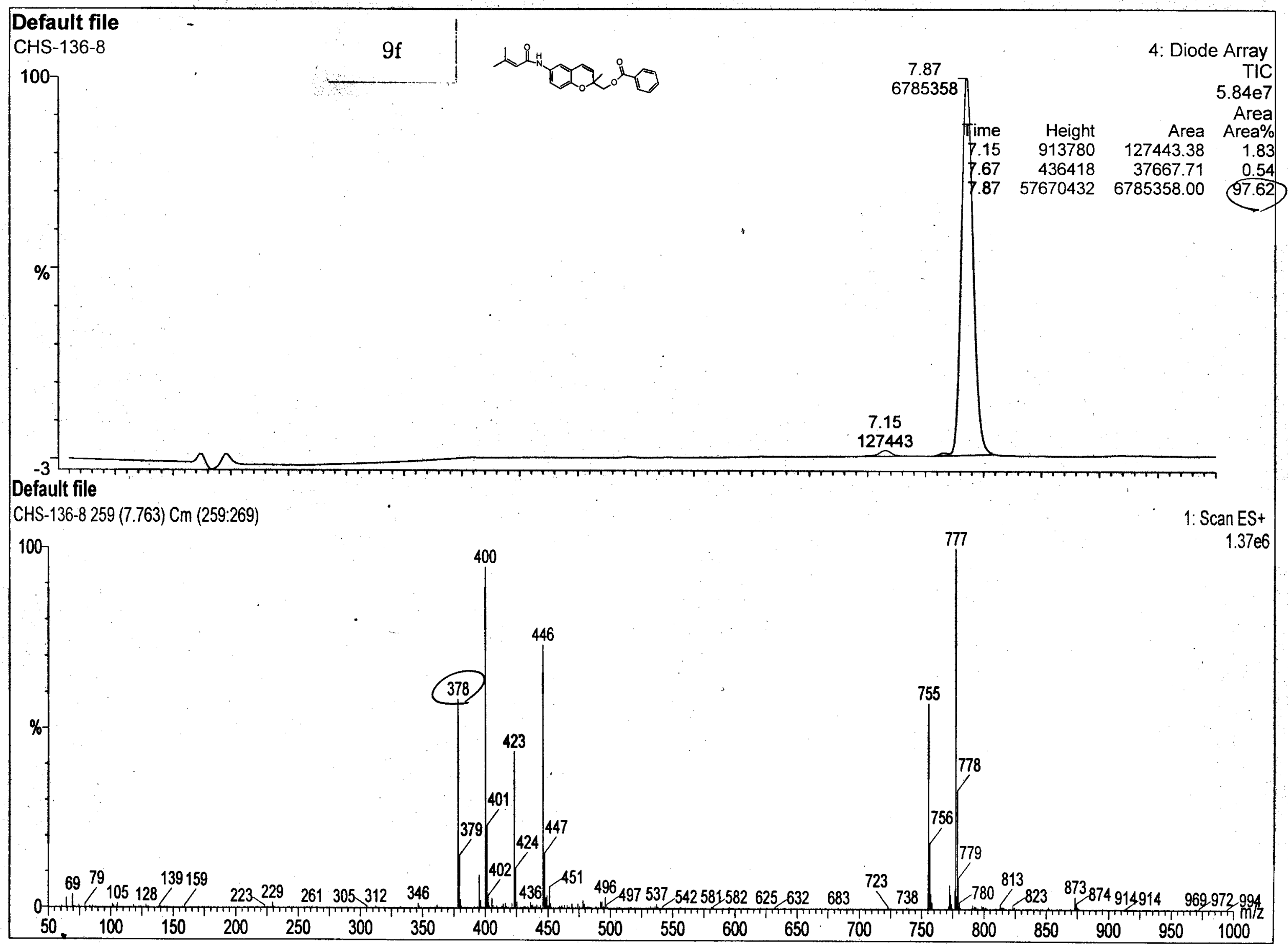


S33

$$
\text { [ } 98 \text {. }
$$

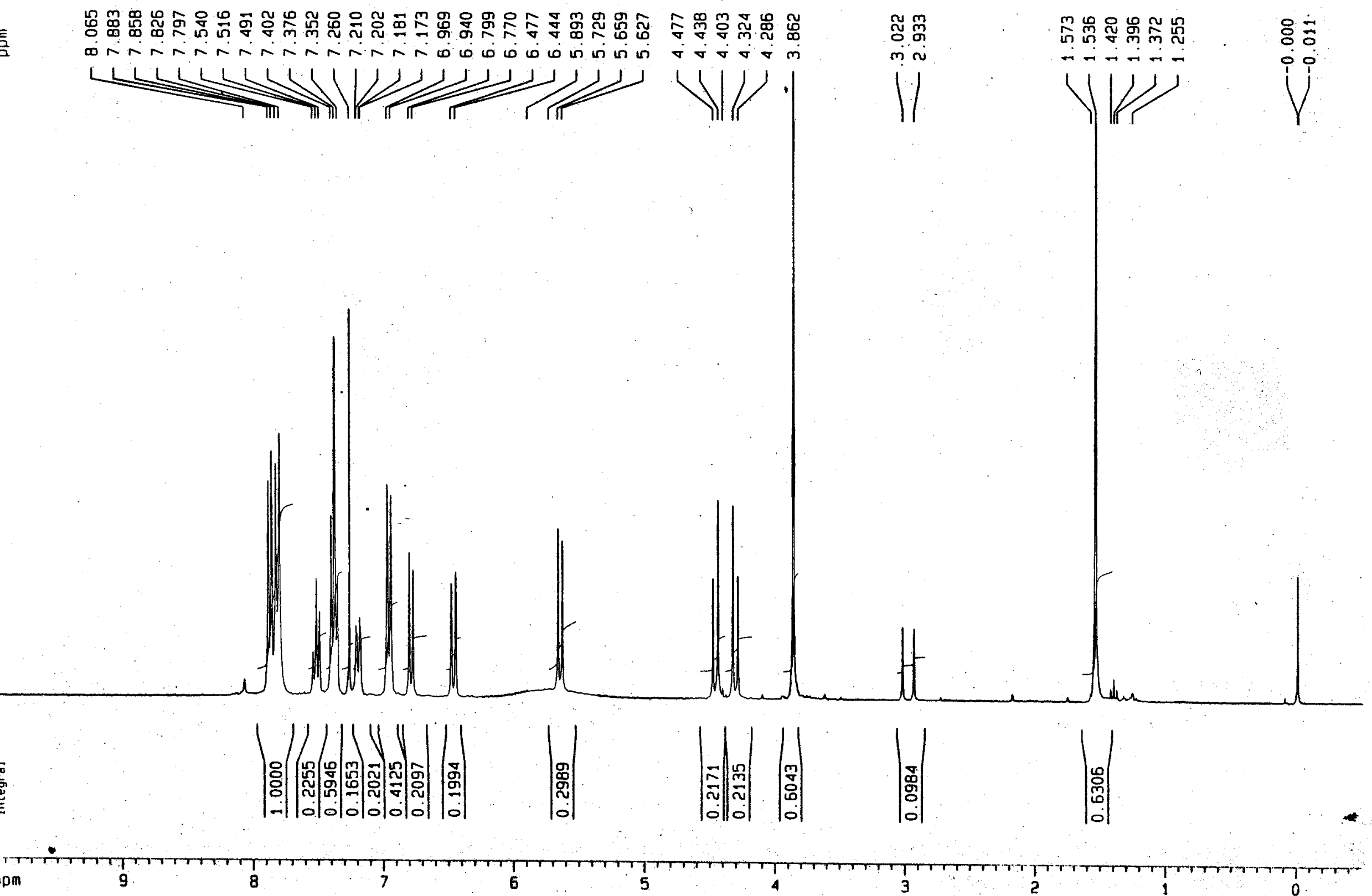




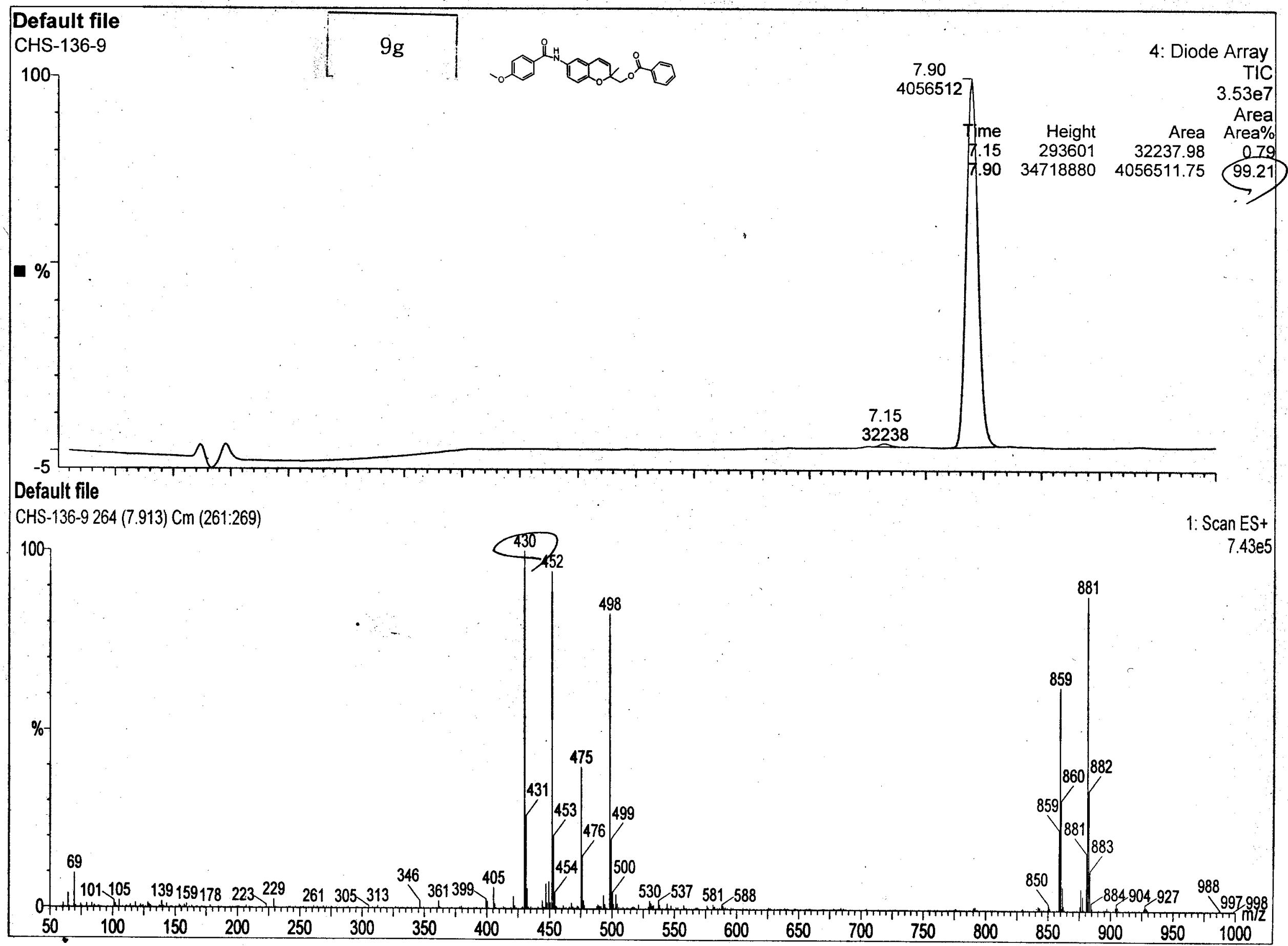




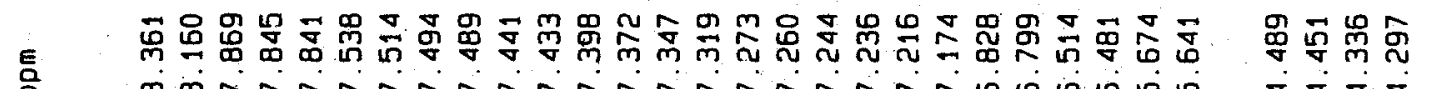
o o $\longrightarrow$

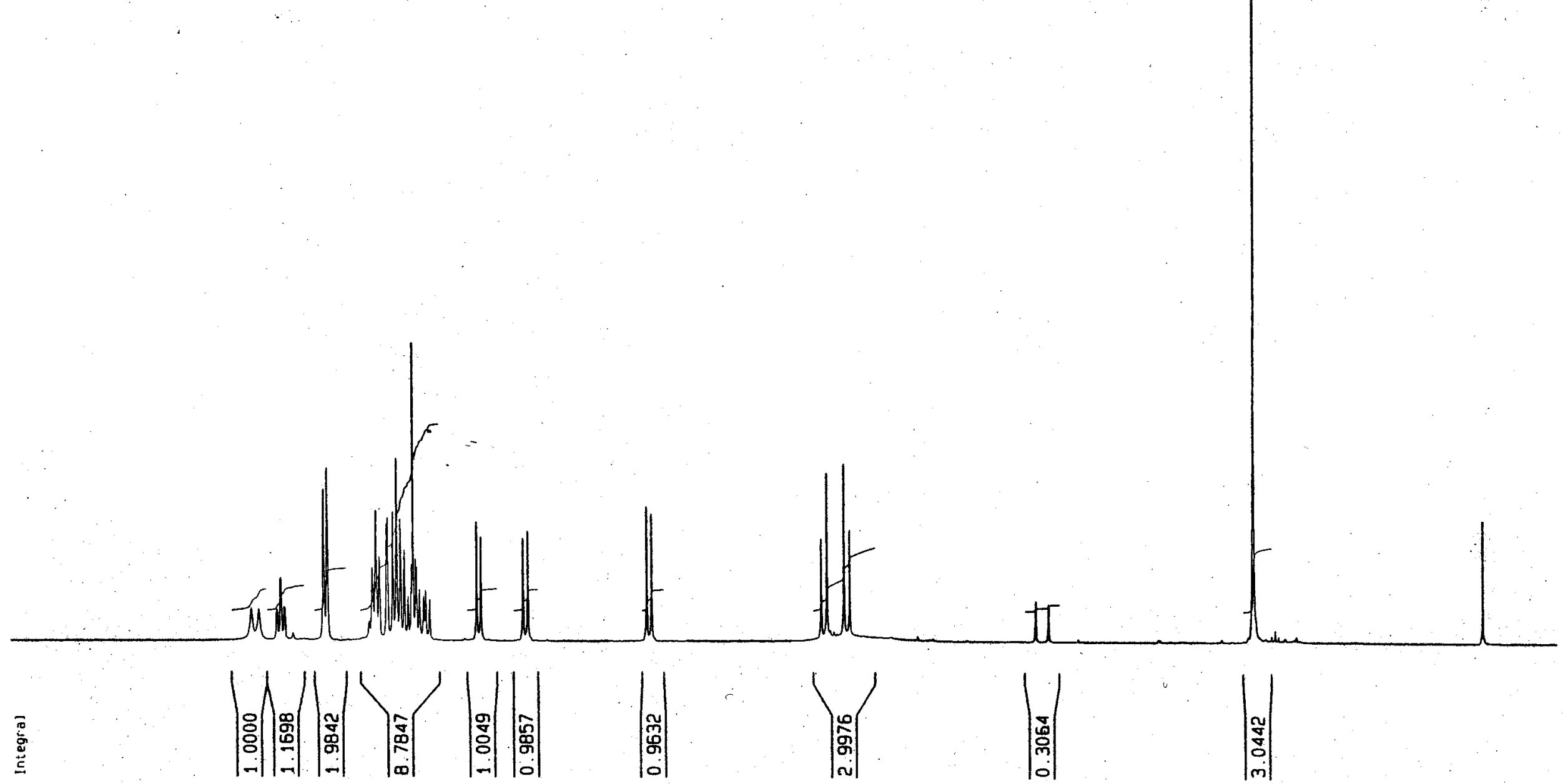




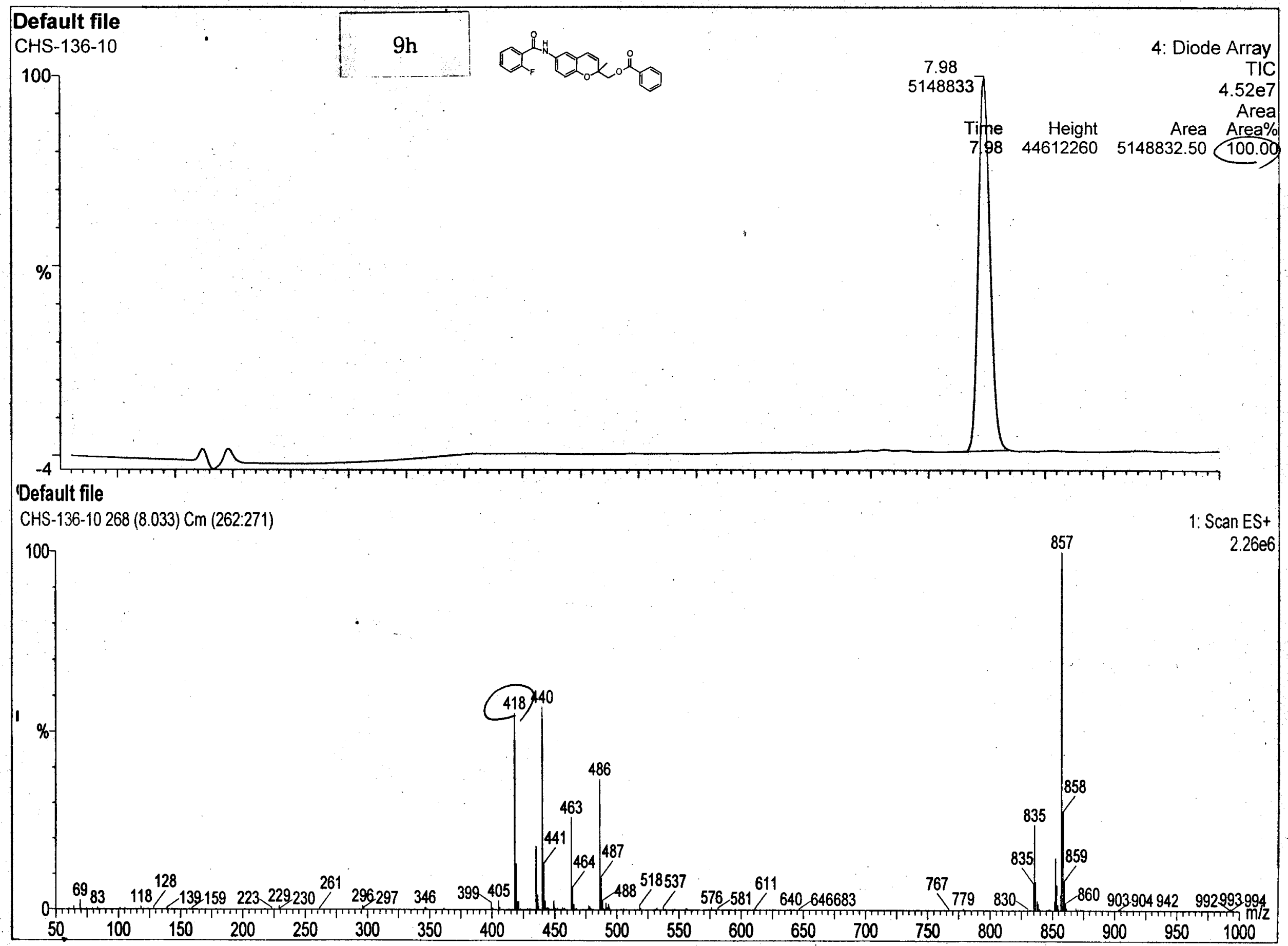




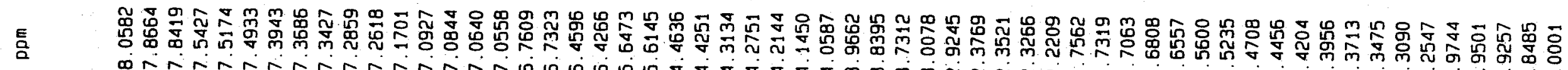

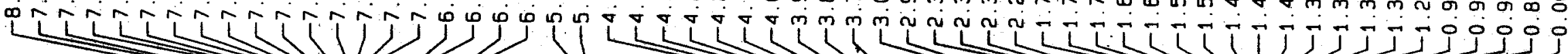
(1) 17 in บाกา $\rightarrow$

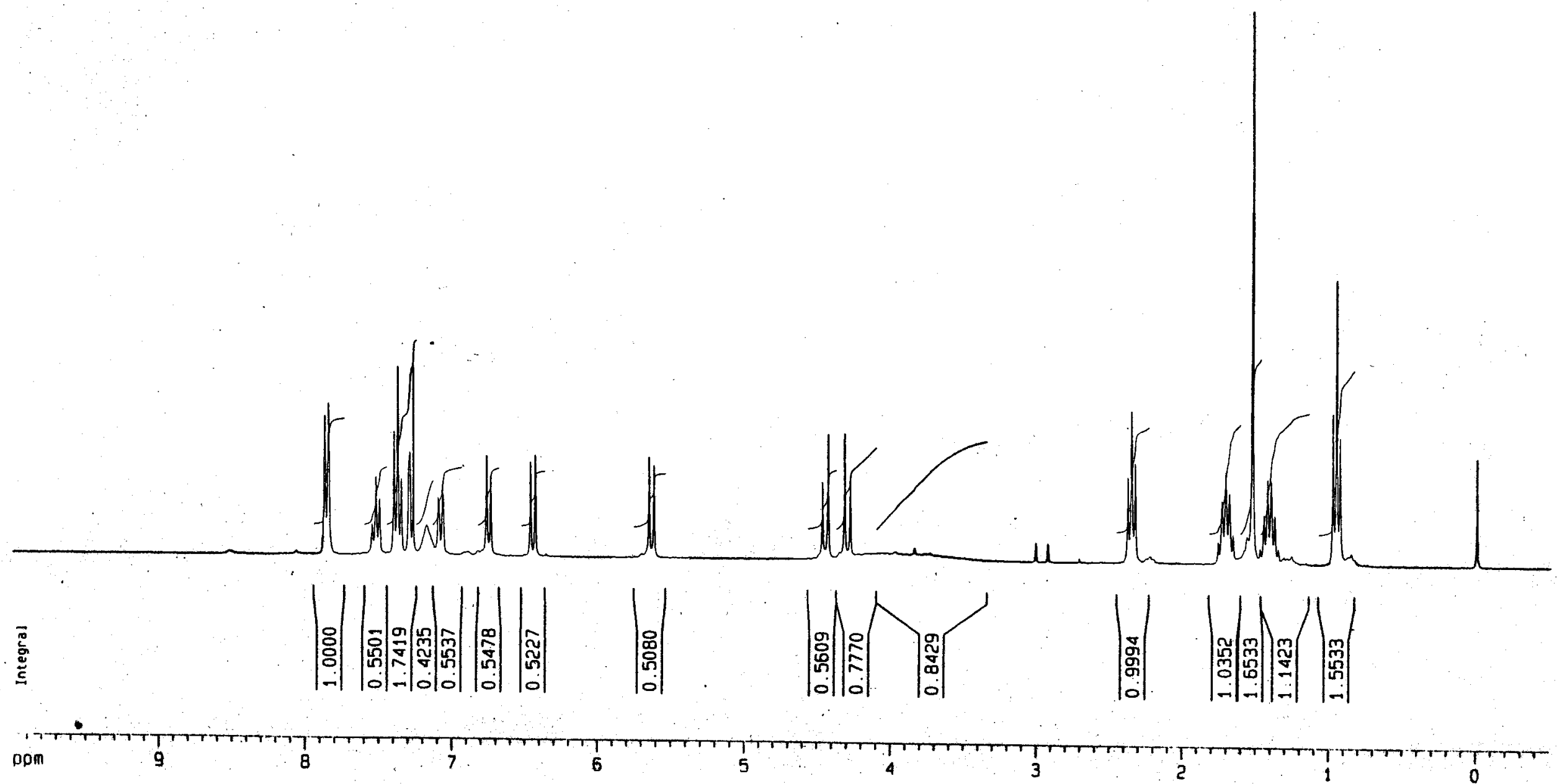




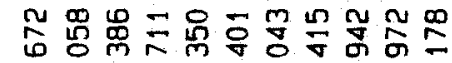

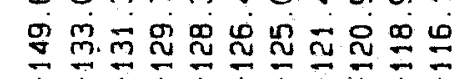
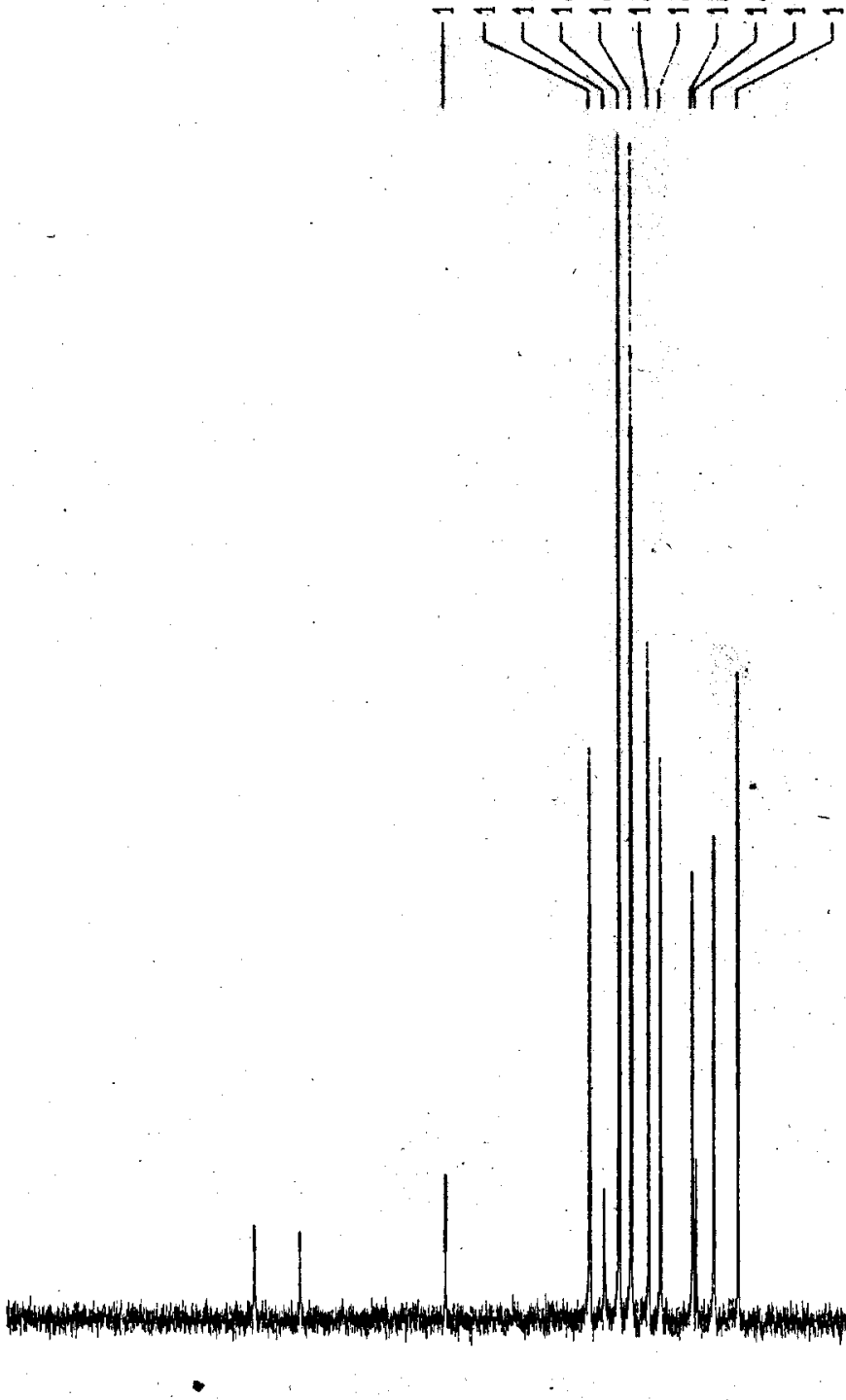

Current Data Parameters

NAME ChS-136-19

SXPNO
PROCNO

$=2$ - Acquisition Parameters

$\begin{array}{lr}\text { Jate- } & 20050712 \\ \text { I } 3 \text { me } & 5.51\end{array}$

INSTRum spect

SROBHD 5 mm our 13C-1

गH PROG
I0

SOL VENT COC I3
VS

35

$\begin{array}{ll}3 \text { WHH } & 35211.270 \mathrm{~Hz} \\ =1 \text { CAES } & 0.537281 \mathrm{~Hz}\end{array}$

AC $\quad 0.5306754 \mathrm{seC}$

$\begin{array}{ll}36 & 114 \\ 3 \mathrm{~W} & 14.200 \text { usec }\end{array}$

14.200 usec
6.00 usec

$29^{7} .0 \mathrm{x}$

2.00000000 sec

$0.03000000 \mathrm{sec}$

1. 89999998 ser

0.01500000 sec

MCWFK

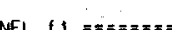

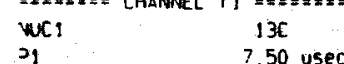

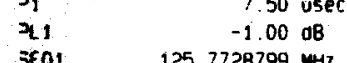

$==3=3=9=$ CHANNEL $+2==3==\pi=4$

ZPDPAG2 waltz16

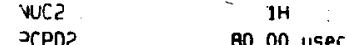

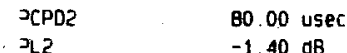

$\begin{array}{ll}2 \mathrm{~L} 2 & -1.40 \mathrm{OB} \\ \mathrm{I}_{12} & 16.66 \mathrm{OB}\end{array}$

$\begin{array}{ll}2 & 12 \\ \mathrm{~L} & 16.66 \mathrm{~dB} \\ \mathrm{~L} 13 & 25.00 \mathrm{~dB}\end{array}$

$\begin{array}{lr}2 \mathrm{~L} 13 & 25.00 \mathrm{~dB} \\ \text { SF } 02 & 500.1320005 \mathrm{HHZ}\end{array}$

=2 - Process ing parameters

$=2$ - Processing parameters
51
32768

$\begin{array}{lr}\text { SI } & 32768 \\ \text { SF } & 125.7577890 \mathrm{MHz}\end{array}$

NOW 125.7577890

$\begin{array}{lc}S S B & 0 \\ B B & 1.00 ~ H z\end{array}$

$x$

10 NMR D Jot parameters

Cx $20.00 \mathrm{~cm}$

$\begin{array}{ll}27 & 12.50 \mathrm{~cm} \\ =1 \mathrm{p} & 200.000 \mathrm{pDm}\end{array}$

$\begin{array}{ll} & 200.000 \mathrm{ppm}\end{array}$

$\begin{array}{ll}=2 \mathrm{P} & -19.995 \mathrm{DPm} \\ =2 & -2514.51 \mathrm{~Hz}\end{array}$

PPMCM $10.99974 \mathrm{DDm} / \mathrm{Cm}$ 


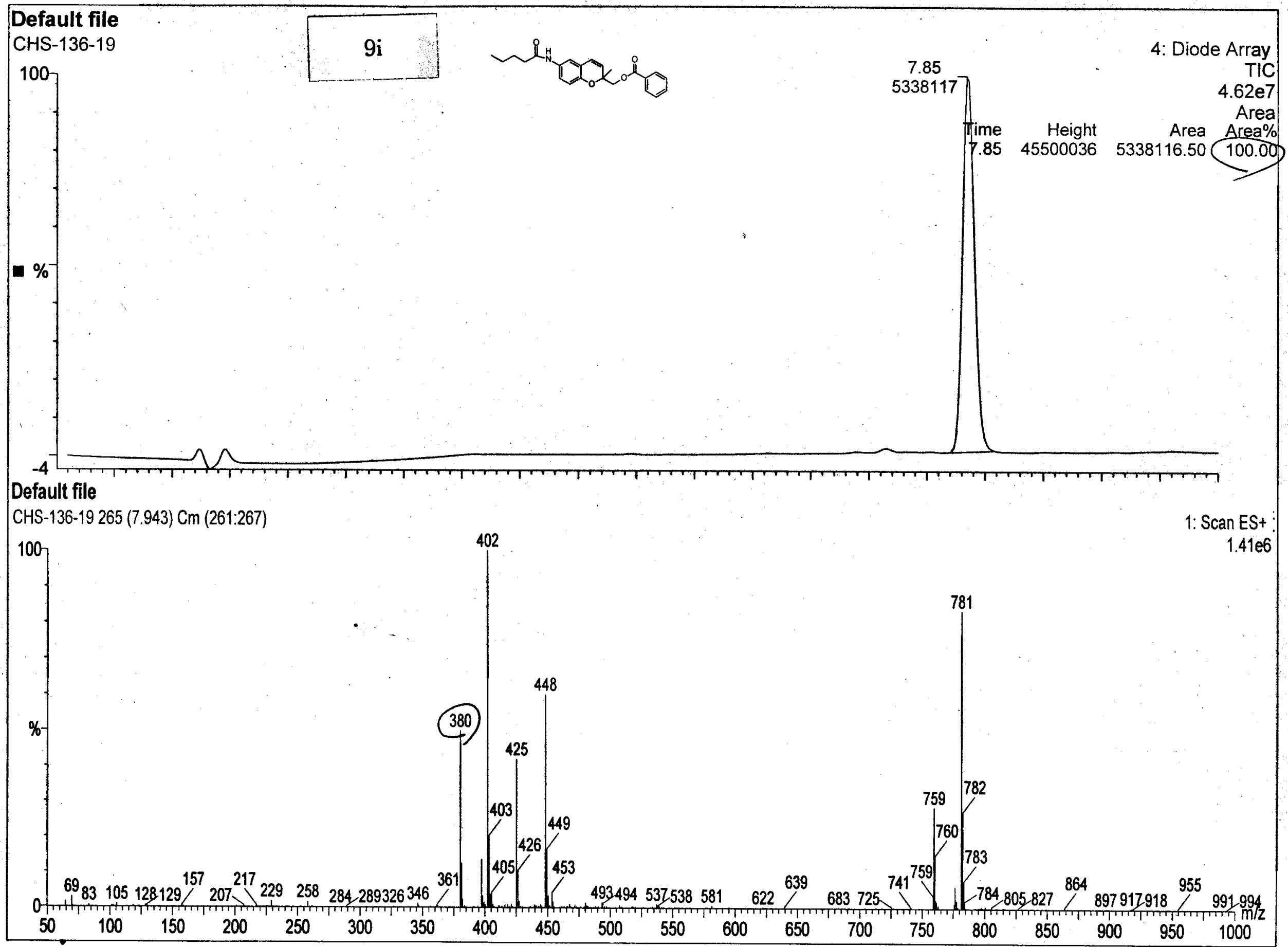



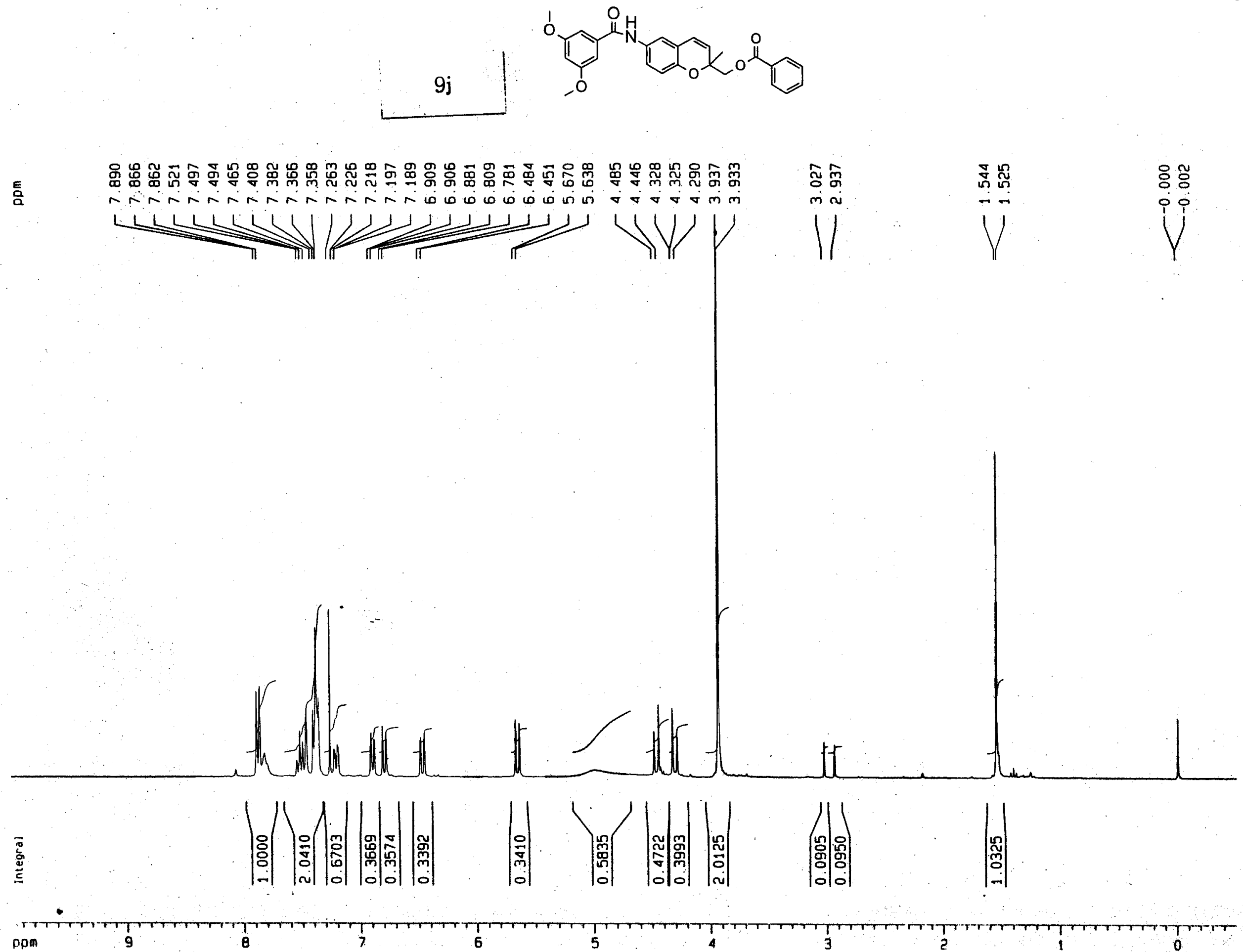


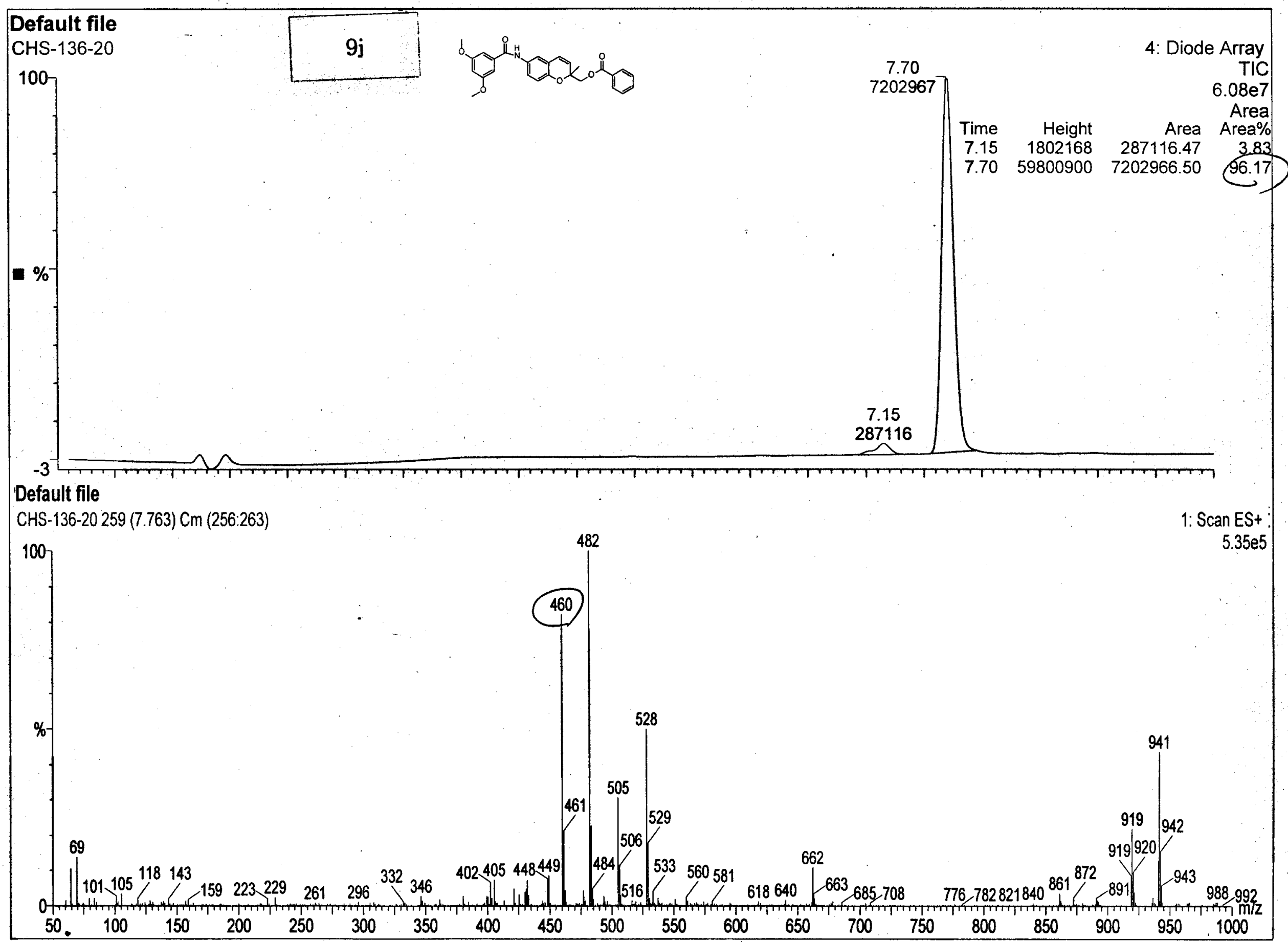




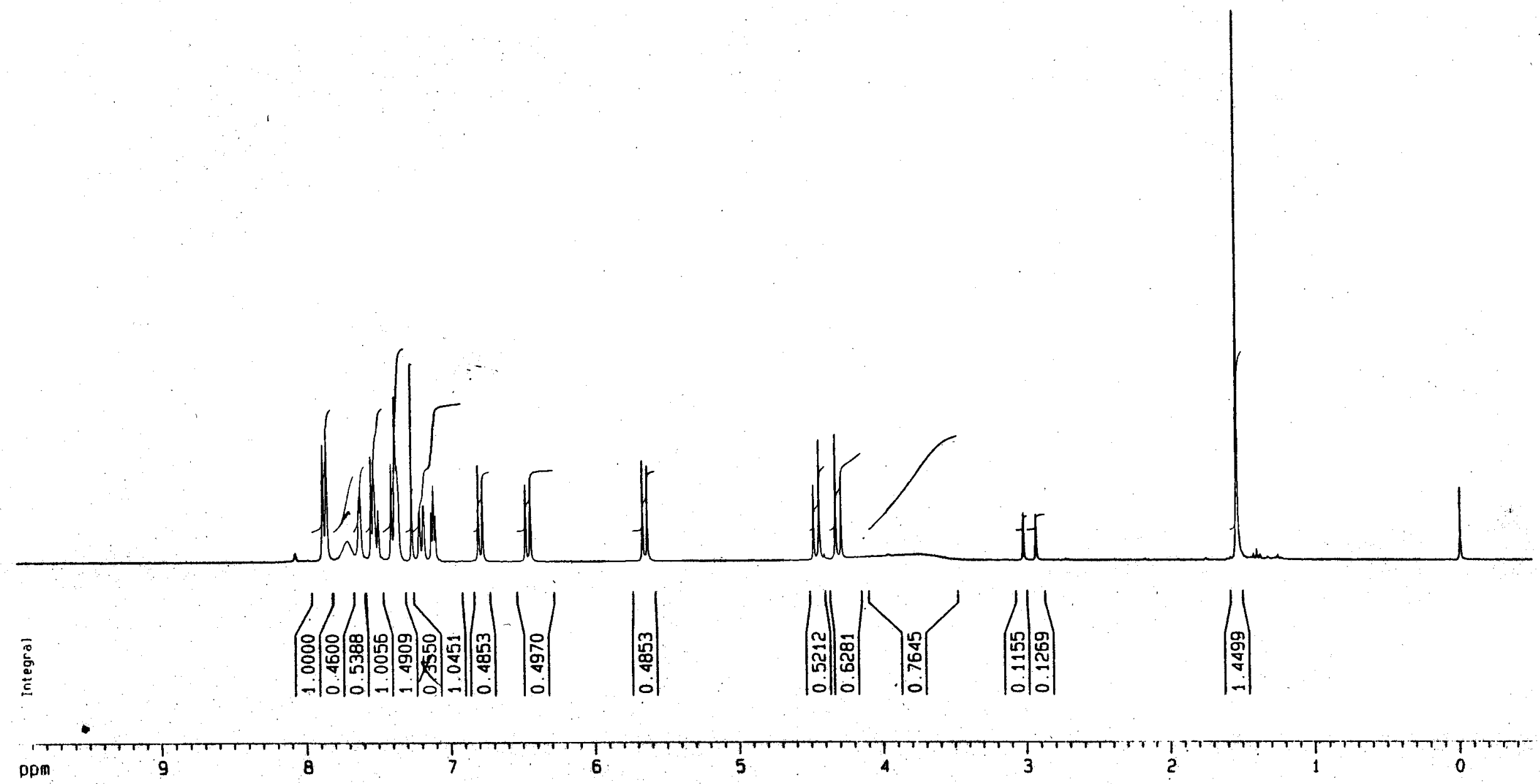




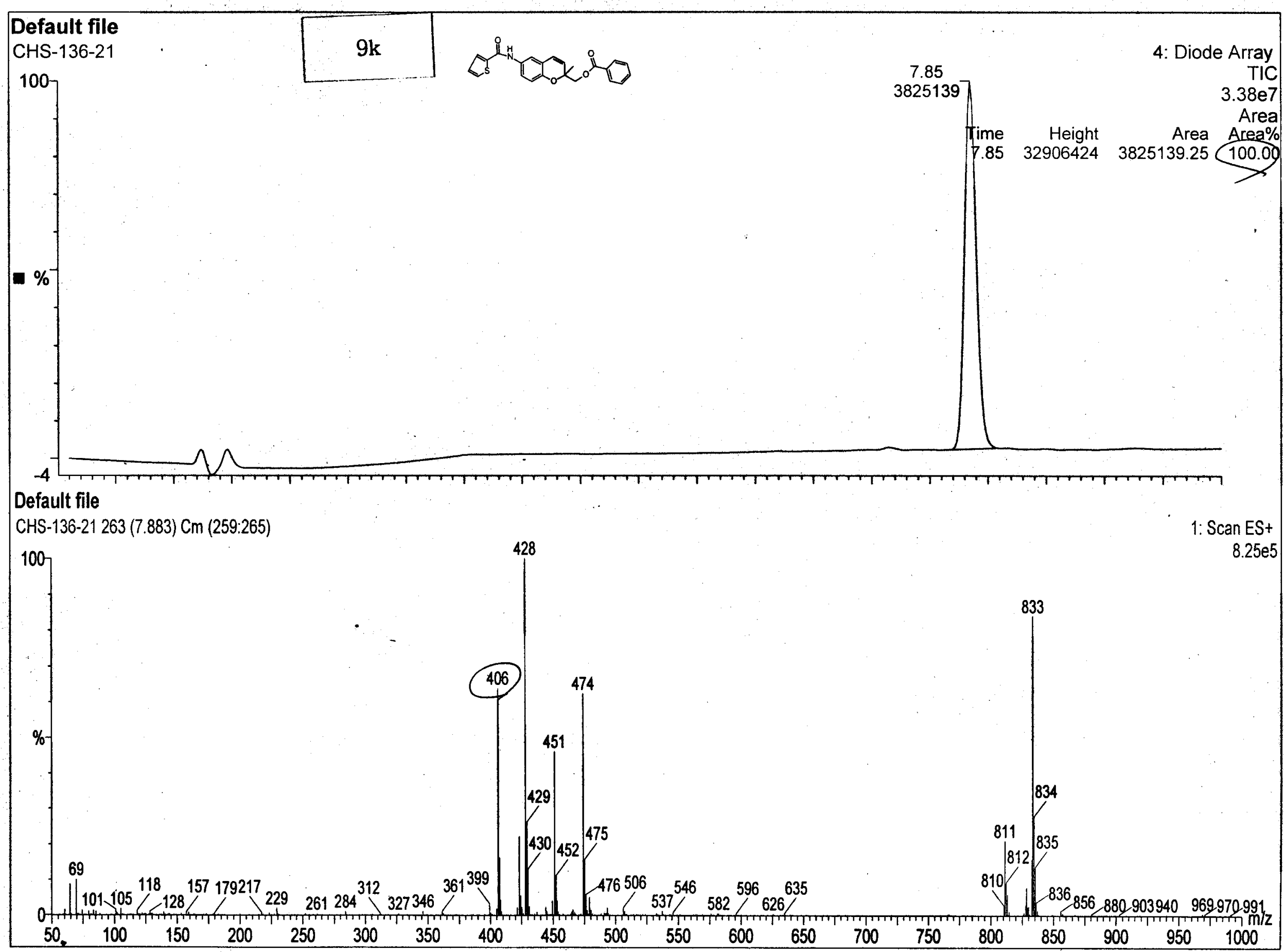



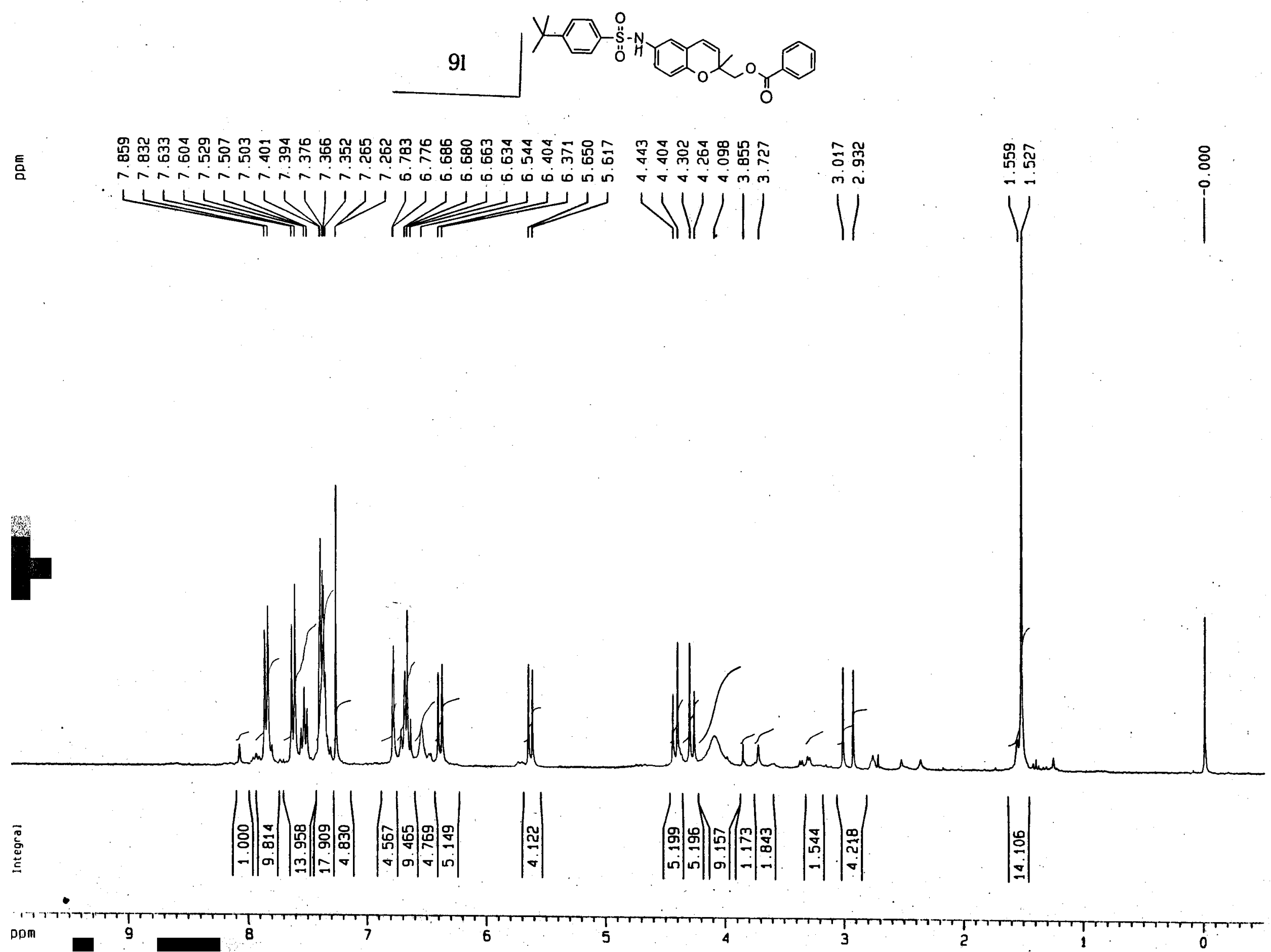


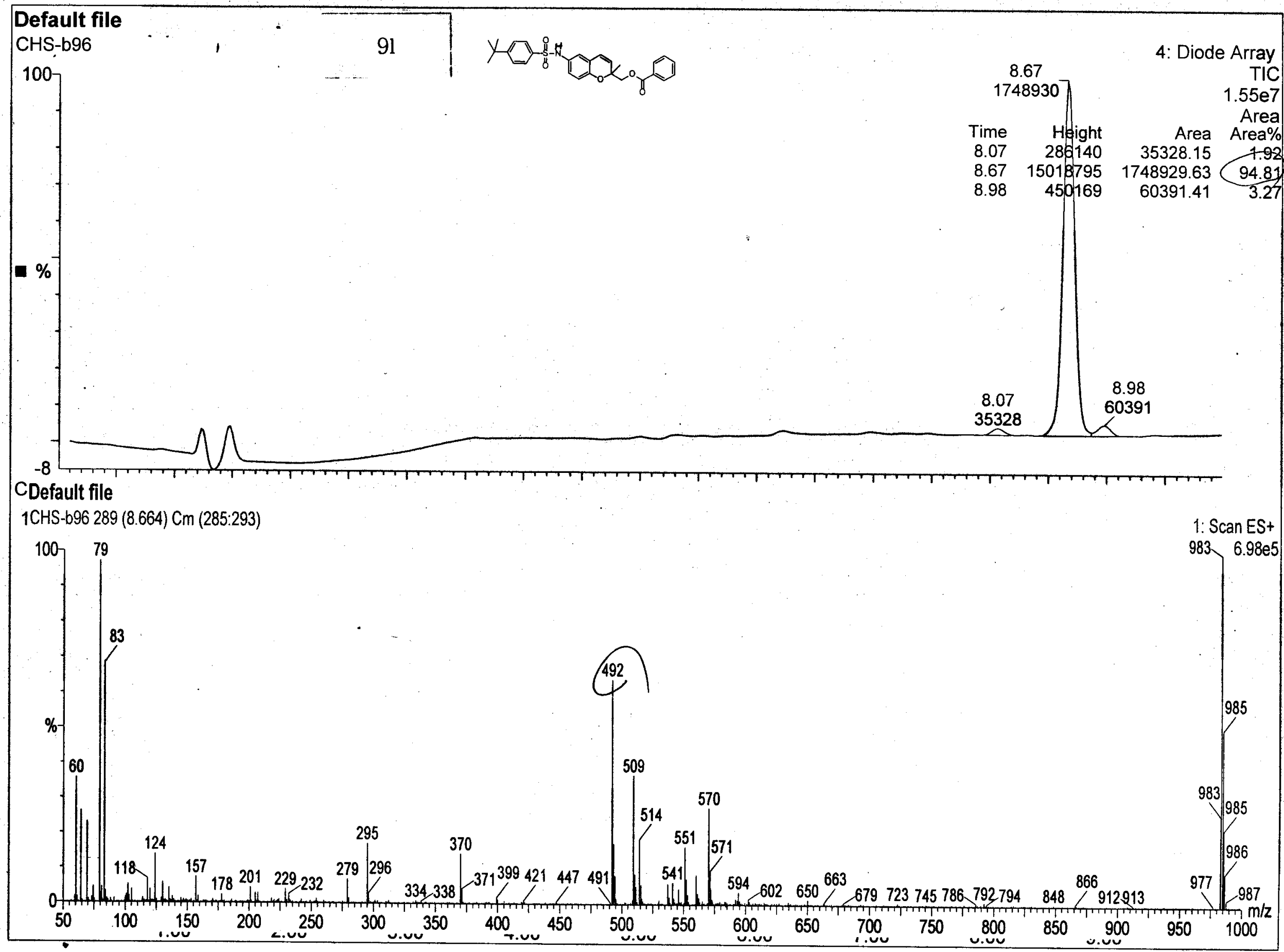



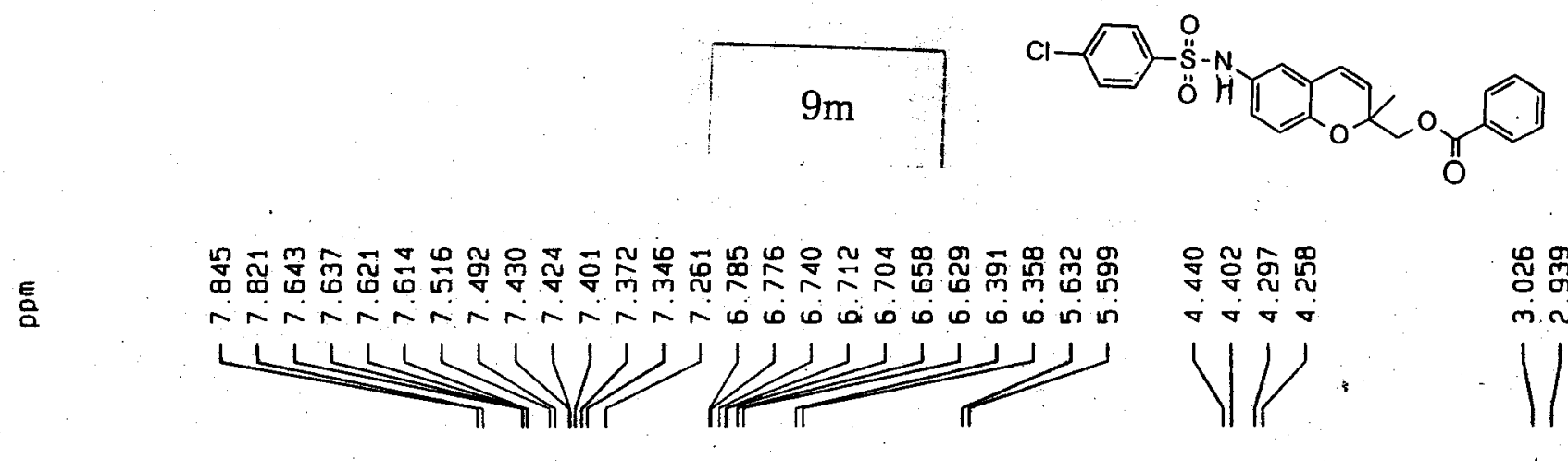

$\mid$
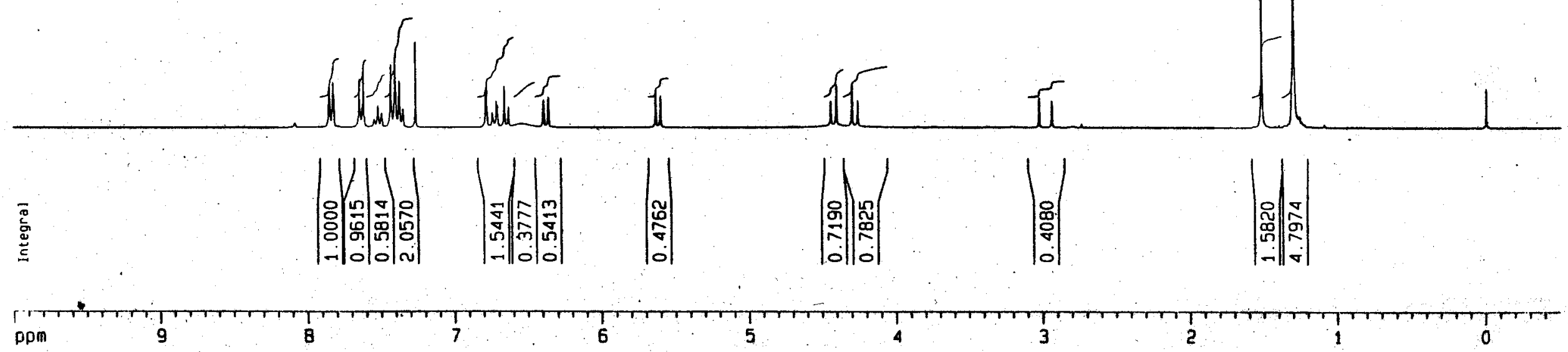


\section{Default file}

CHS-b97(CC)

100

$9 \mathrm{~m}$

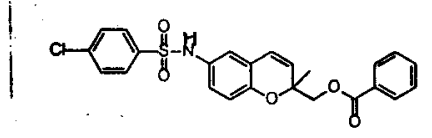

8.25

847032

4: Diode Array

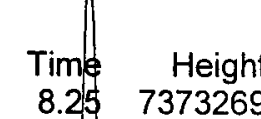

8.257373269

8.581018614

$\begin{array}{ll}1018614 & 117105.42\end{array}$

$7.72 \mathrm{e} 6$
Area

Area Area\%

$117105.42 \quad \begin{aligned} & 87.85 \\ & 42.15\end{aligned}$

$\%$

$\%$

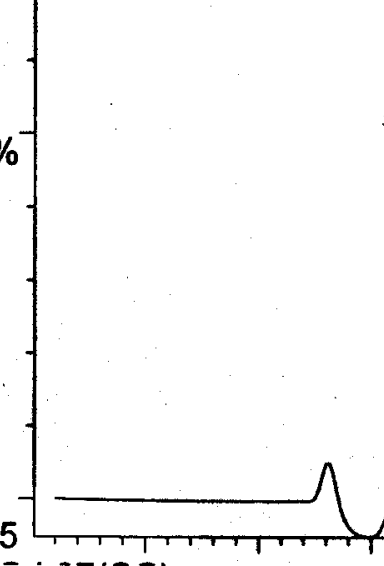

Default file

${ }^{1} \mathrm{CHS}-b 97$ (CC) 278 (8.334) $\mathrm{Cm}$ (273:281)

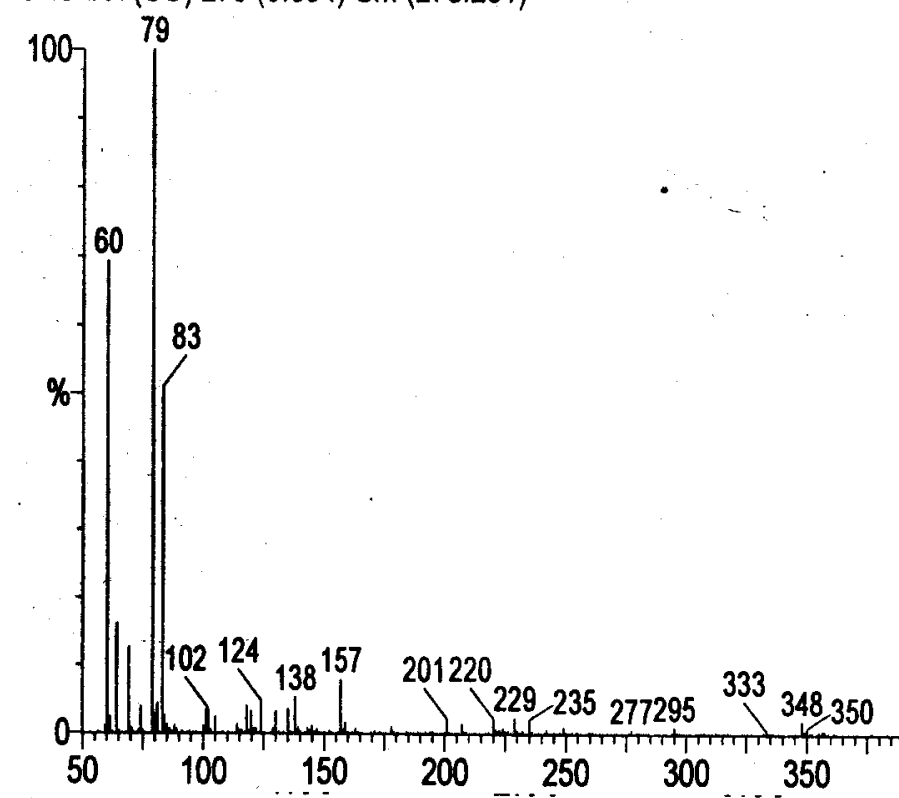

399

421

$400 \quad 470{ }^{487} \quad 529548550573 \quad 627630693$ $529548550,573 \quad 627630$ 693703 


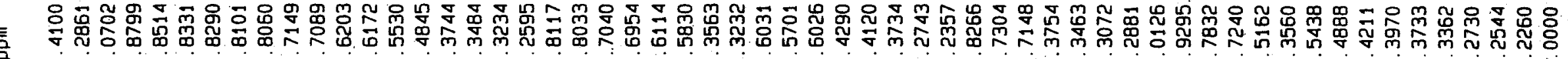

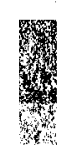
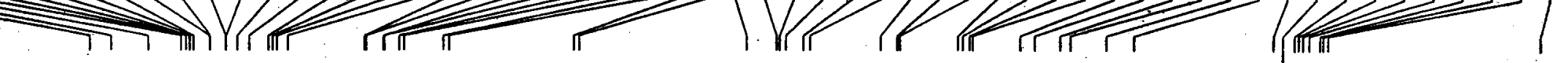

䤈
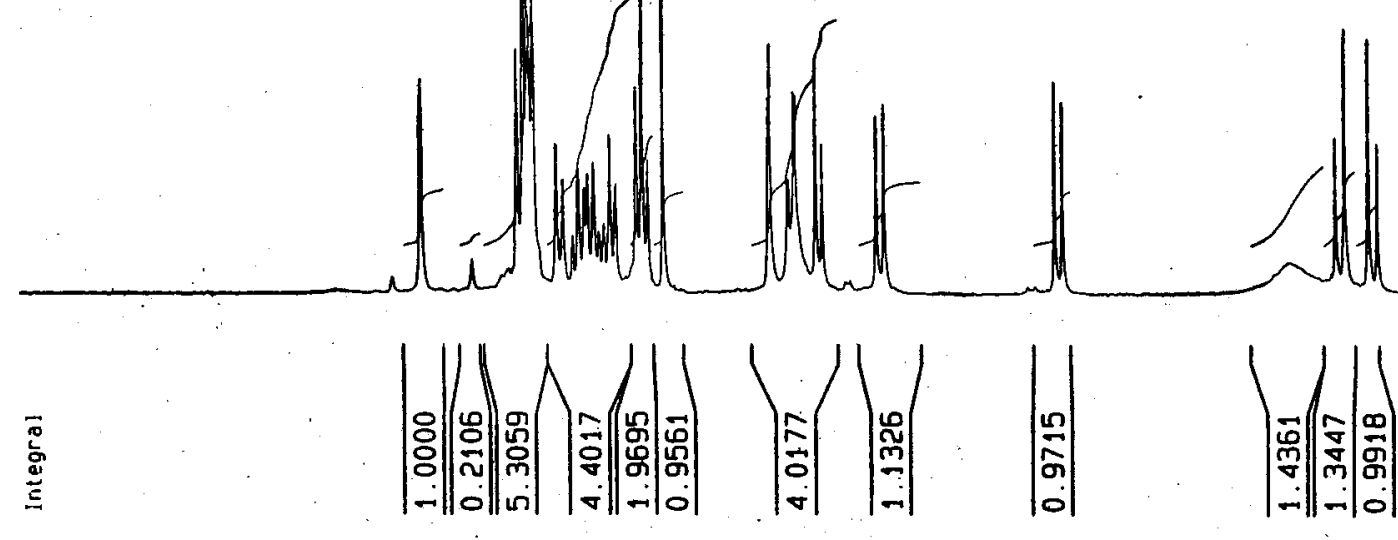

$\mid$

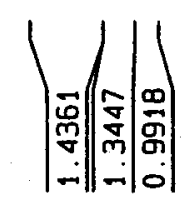

$\left|\begin{array}{l}\mid \\ 0 \\ 0 \\ 0\end{array}\right|$

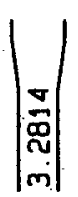




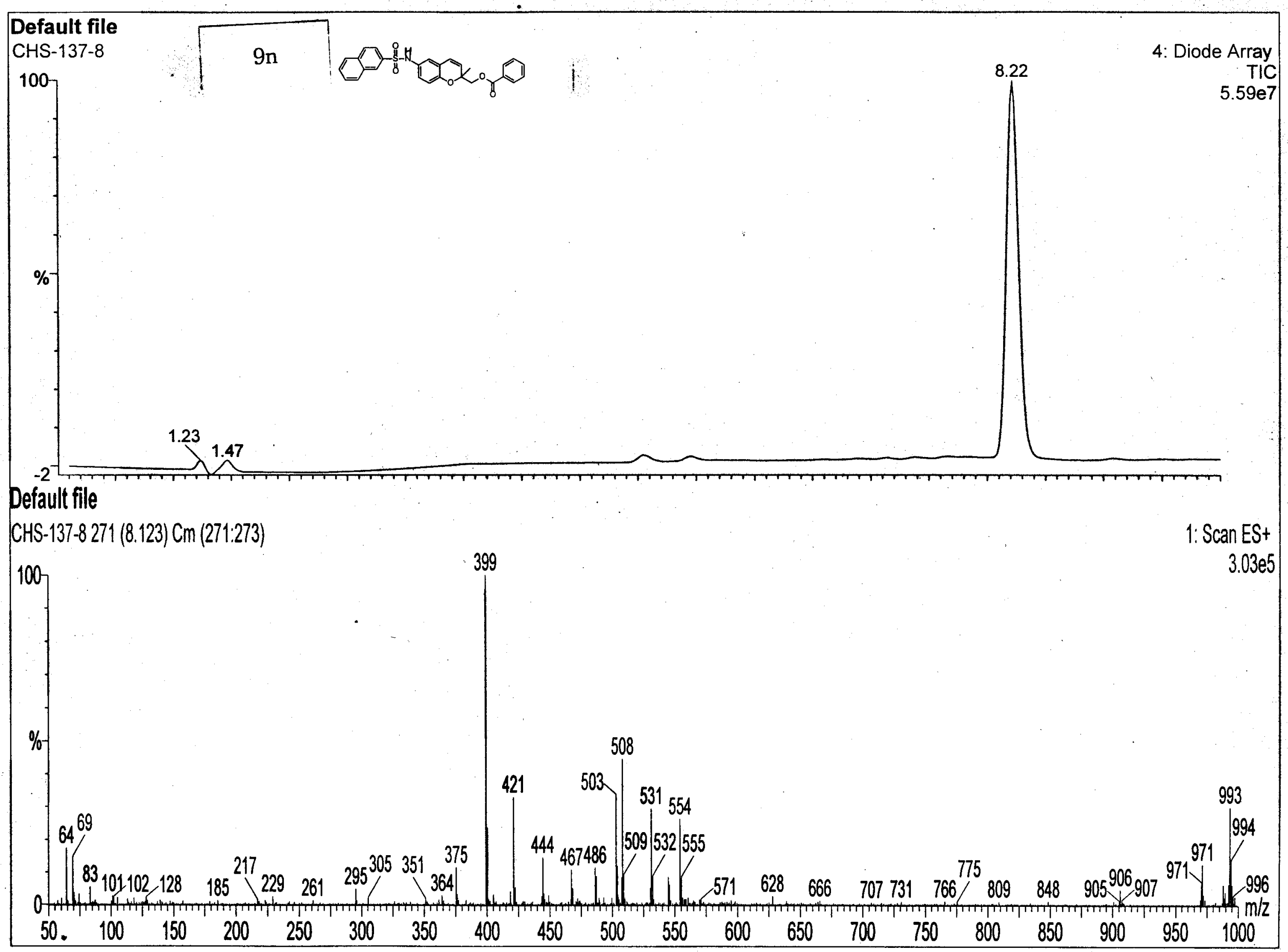




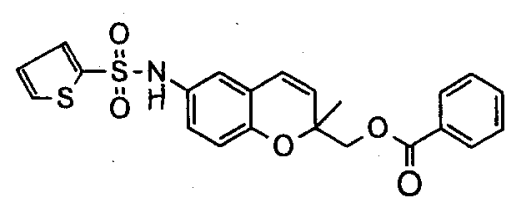

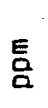
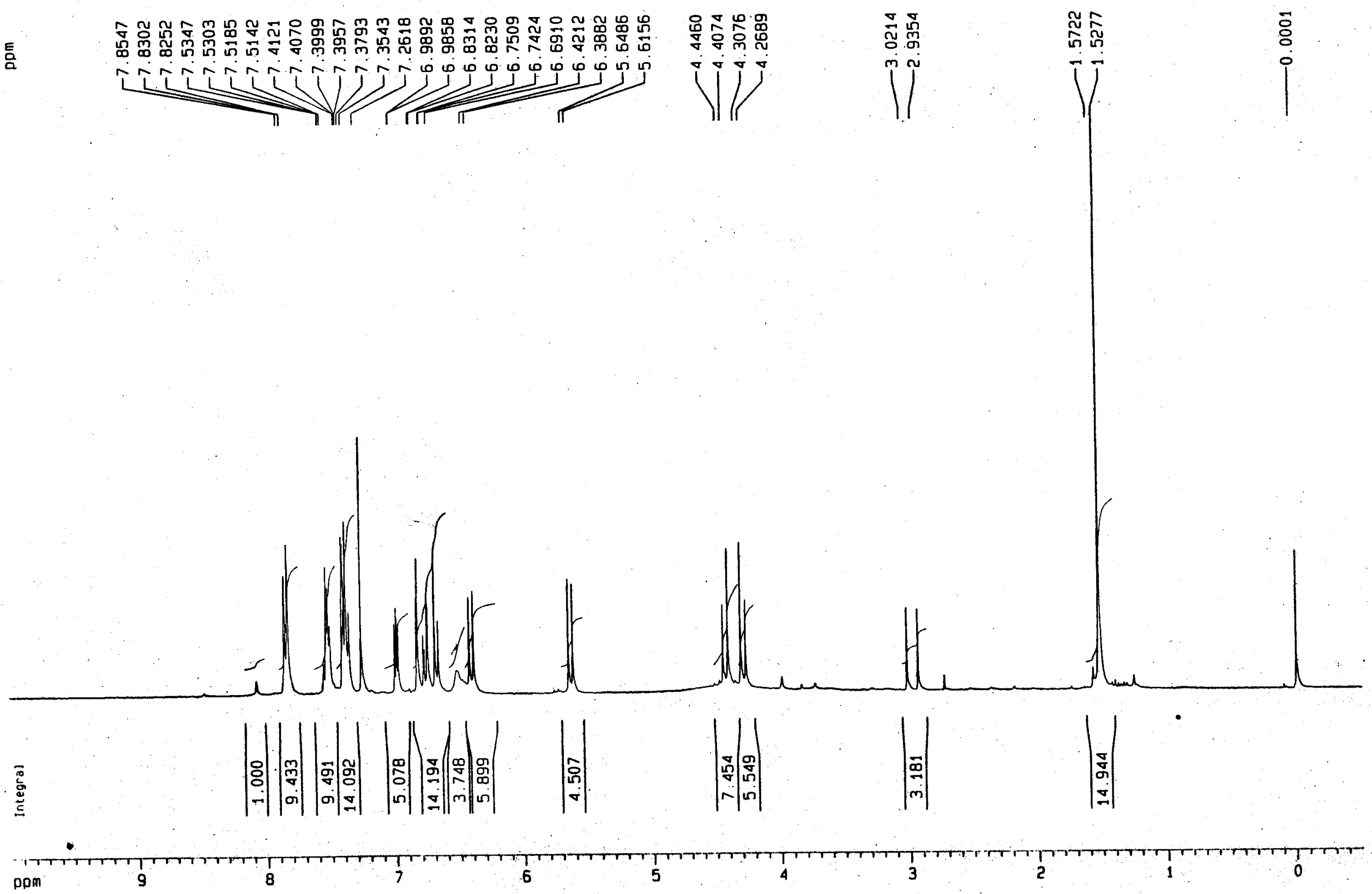


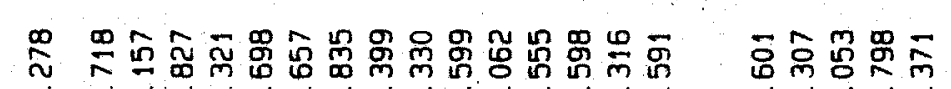

.
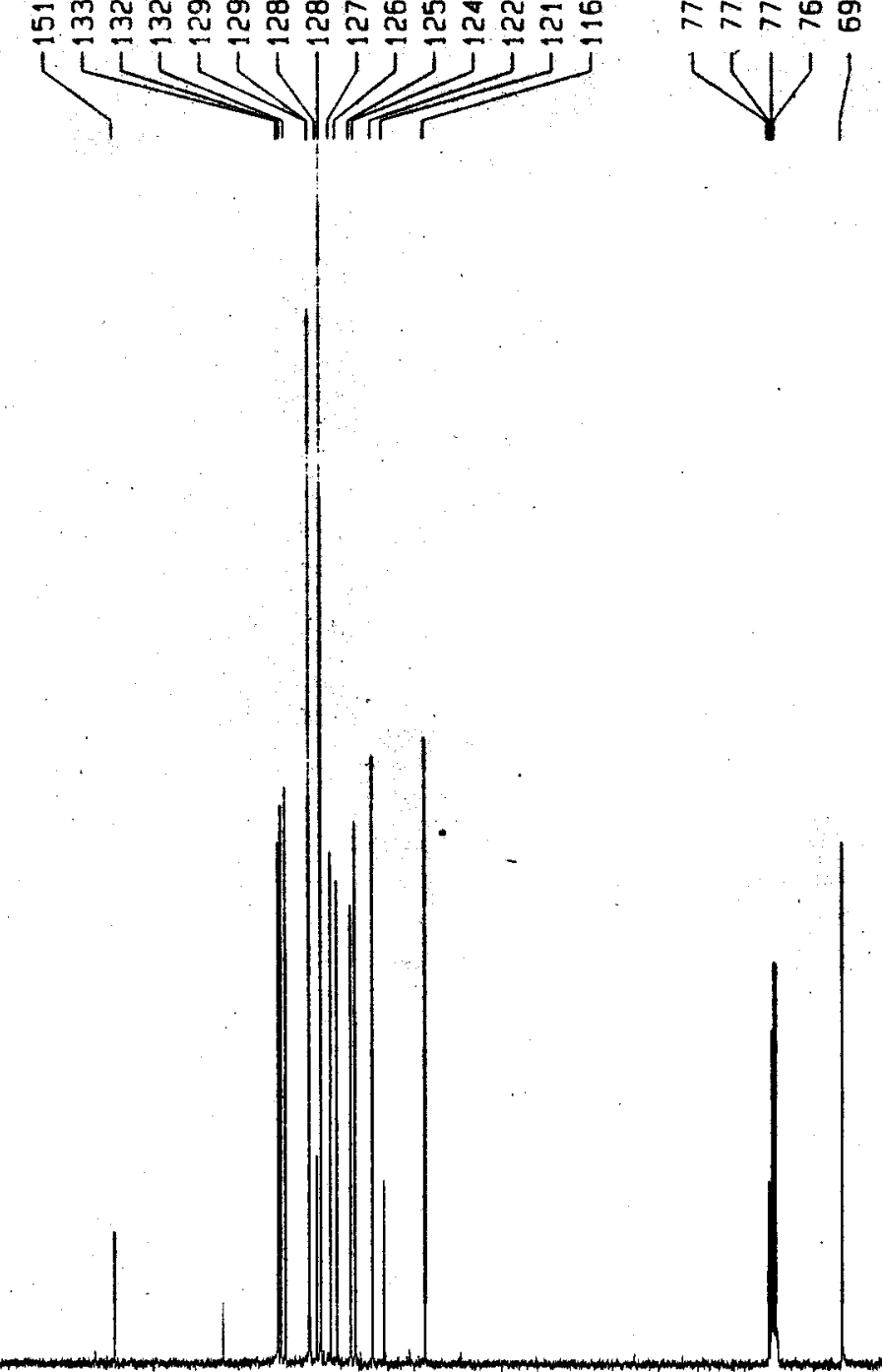

Current Data Parameters

NAME CHS-137-12

EXPNO

PROCNO

$=2$ - Acquisition Parameters

Jate_ 20050714

Time

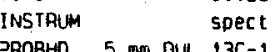

2909
65536

SOL VENT

25

$35: 2: 2 \% 0$

$0.537201 \mathrm{mz}$
$0.9306754 \mathrm{ser}$

76

IN 14.200 usec

6.00 uses

$29 \div x$

10.000000 set

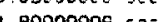

$0.00000000 \mathrm{sec}$

$001500000 \mathrm{SEC}$

Hannel if $x=z=x:=z$

WeI 135

$31 \quad 750$ usec

$\begin{array}{lr}{ }_{21} & -1.00 \mathrm{dE} \\ \mathrm{SFO1} & 125.7728799 \mathrm{NHz}\end{array}$

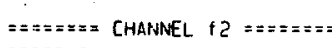

SPDPRG Waltz16

$\begin{array}{ll}\text { VUC2 } & 1 \mathrm{H} \\ \text { PCPO2 } & 80.00 \text { usec }\end{array}$

$\begin{array}{ll}\mathrm{L} 2 & -1.40 \mathrm{~dB}\end{array}$

25.60000
0132

$5 F 02 \quad 500.1320005$ HHZ

2 - Processing parameters

$31 \quad 32768$

$\begin{array}{lr}\text { SI } & 32768 \\ \text { SF } & 125.7577890\end{array}$

NOW
SSB

$\begin{array}{cc}-B & 1.00 \mathrm{H} \\ G B & 0\end{array}$

10 NMA plot parameters
$2 x \quad 20.00 \mathrm{~cm}$
$2 x$

$\begin{array}{ll}\mathrm{cx} & 20.00 \mathrm{~cm} \\ \mathrm{cr} & 12.50 \mathrm{~cm}\end{array}$

FIP $\quad 200.000 \mathrm{ppm}$

$\begin{array}{ll}\text { F1 } & 25151.56 \mathrm{~Hz} \\ \text { F2P } & -19.996 \mathrm{opm}\end{array}$

F2 $\quad-2514.69 \mathrm{~Hz}$

DPMCM $10.99982 \mathrm{ppm} / \mathrm{Cm}$

$383.31262 \mathrm{~Hz} / \mathrm{cm}$ 


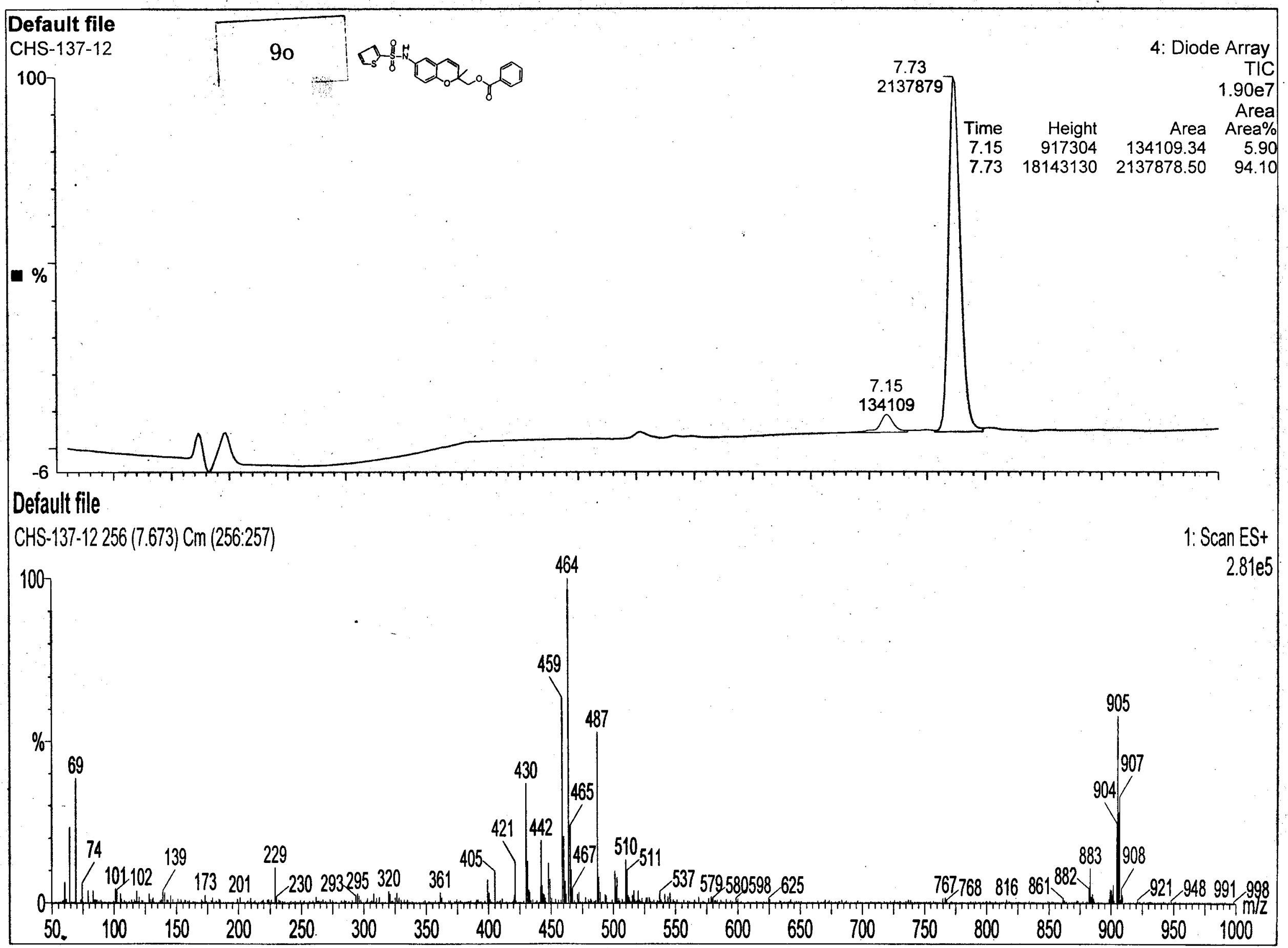




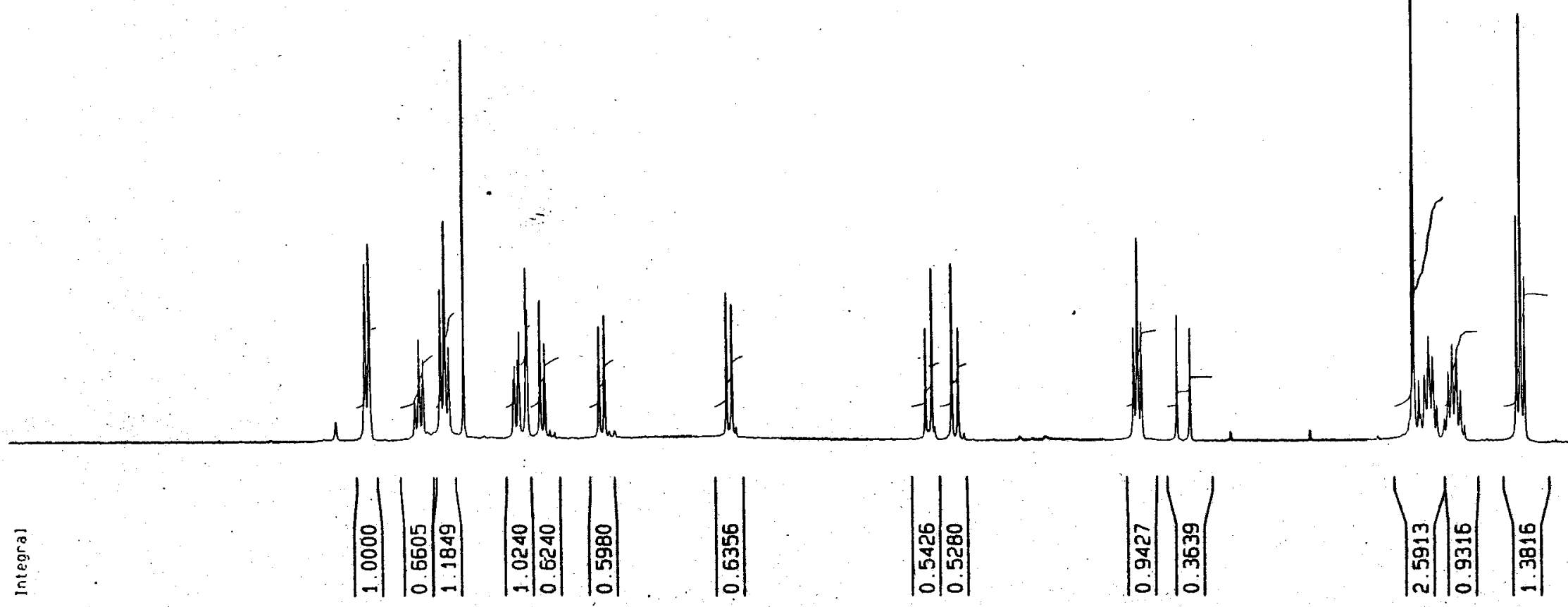




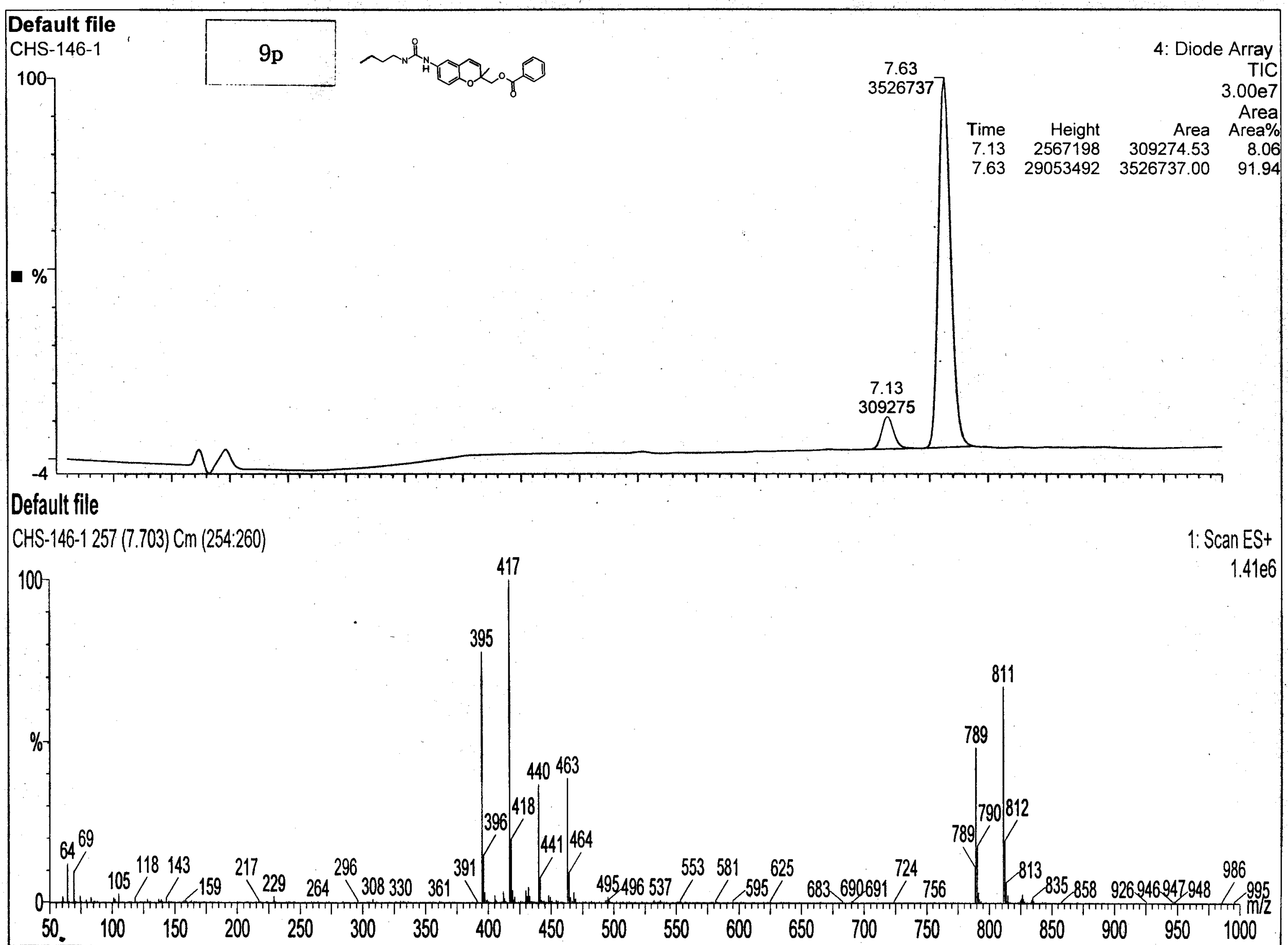




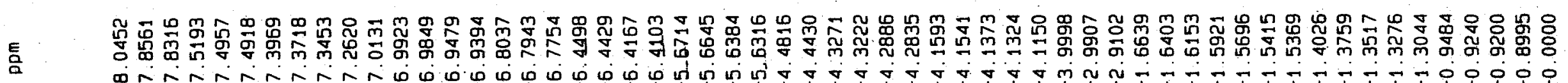
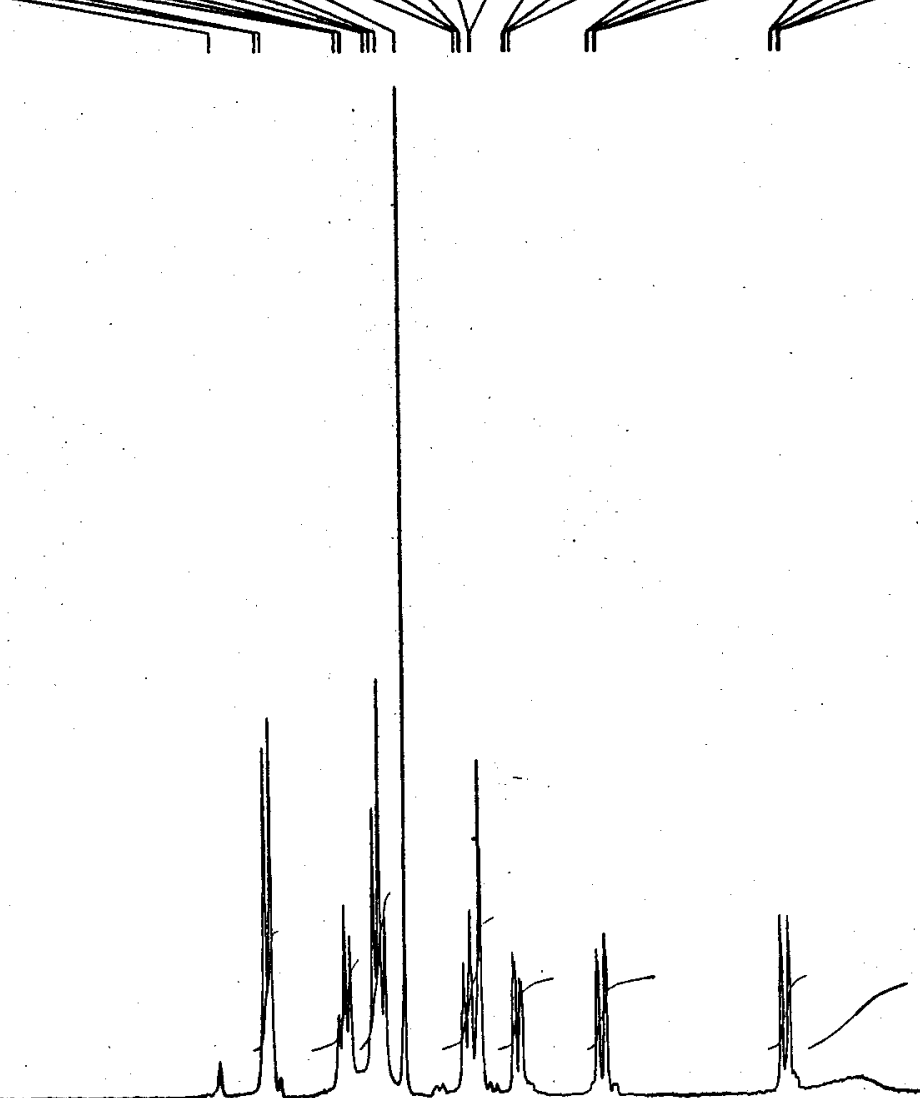


\section{Default file}

CHS-146-2

1007

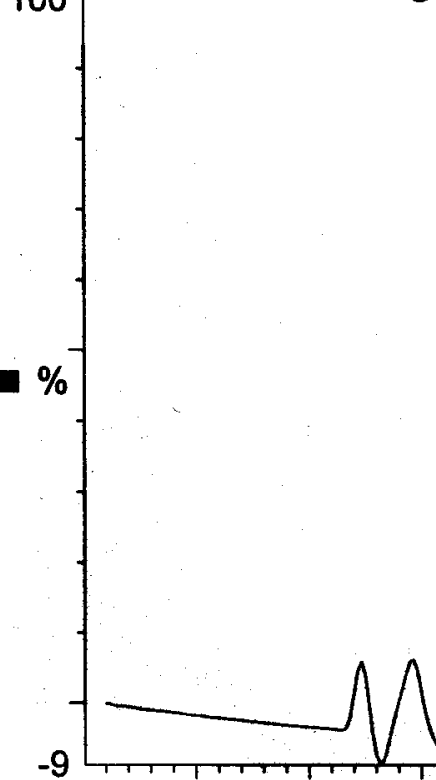

Default file

CHS-146-2 258 (7.733) Cm (255:259)

100

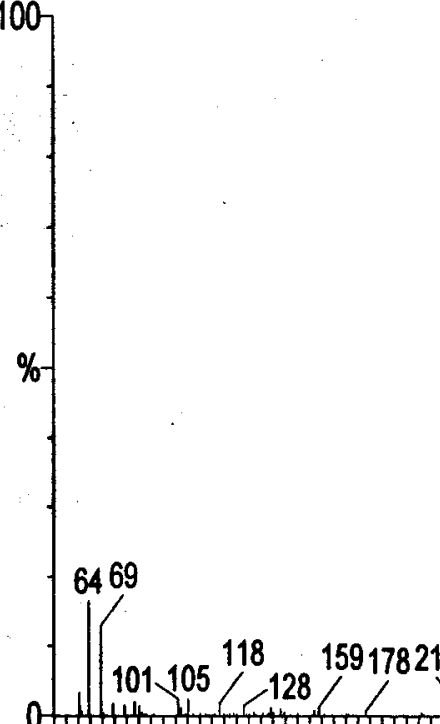

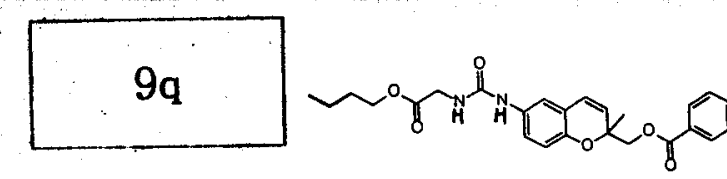
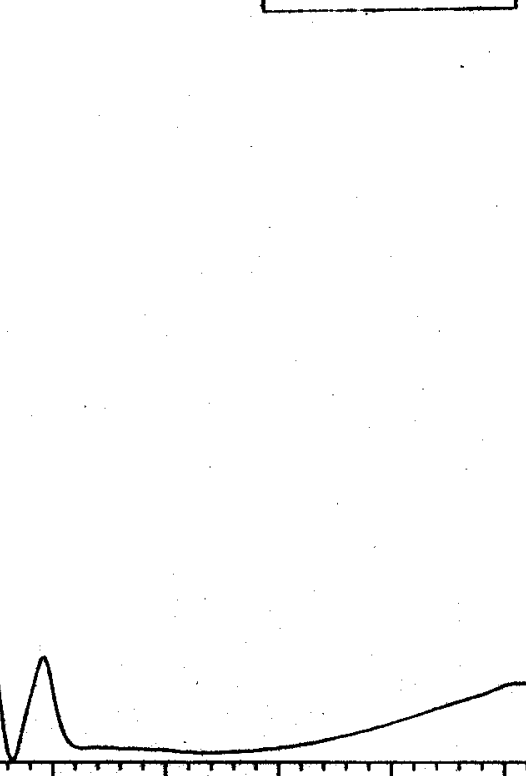
9)
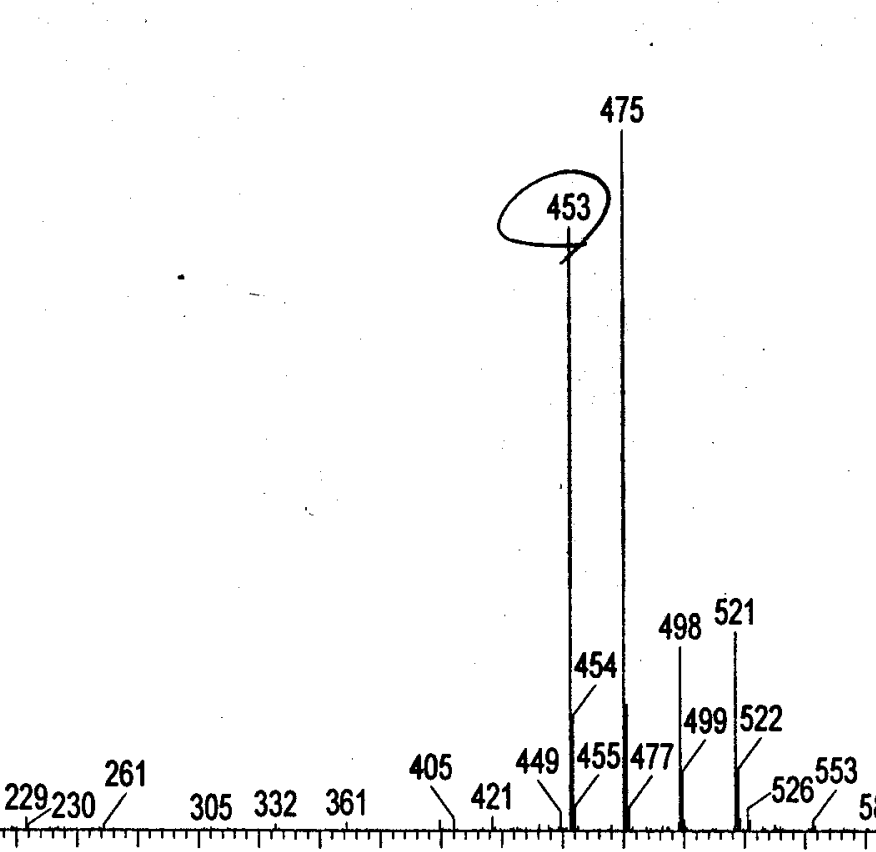

$26^{553}$

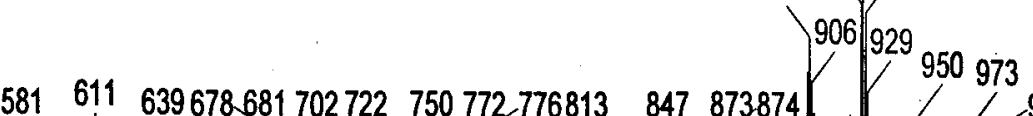
$305332 \quad 361$ 

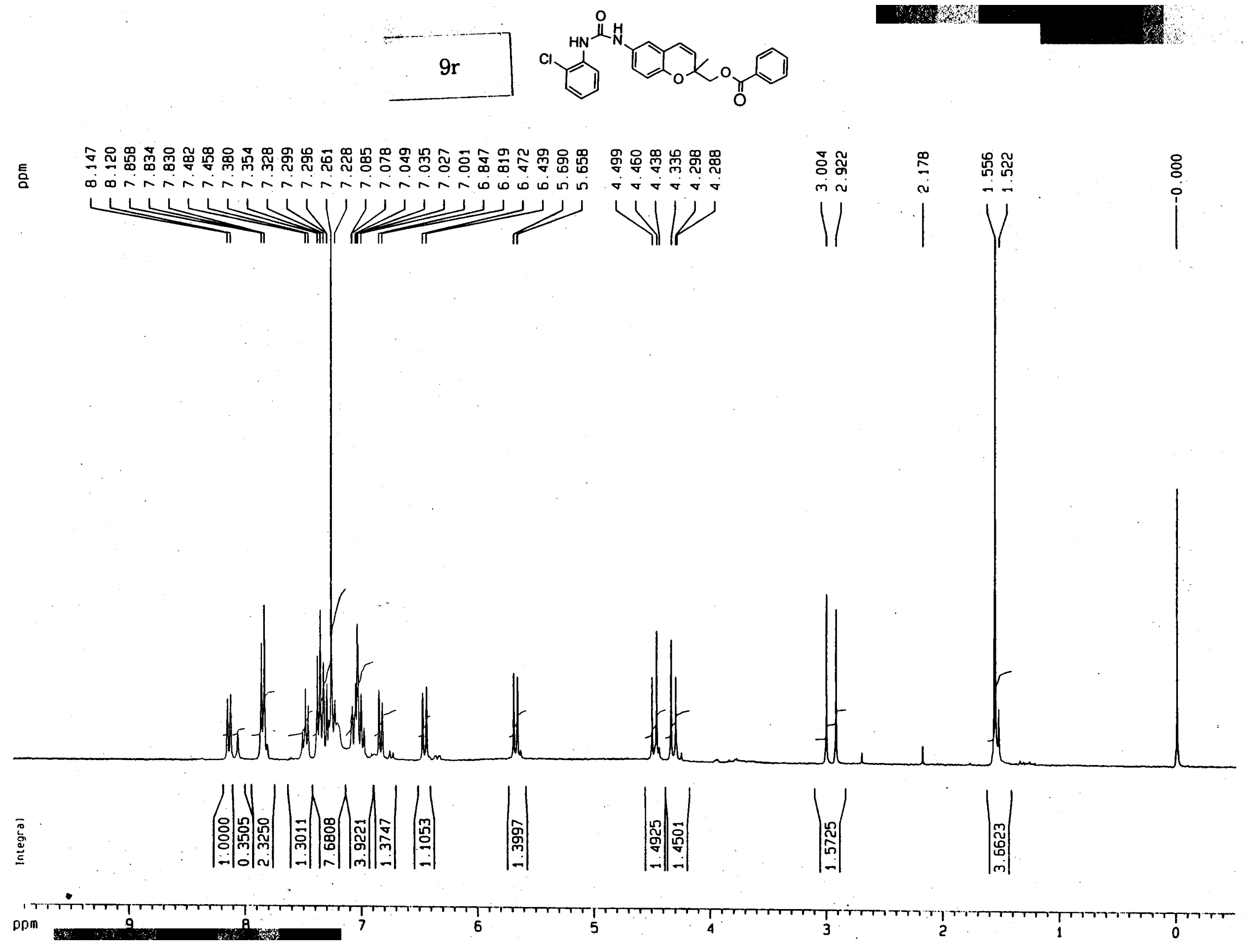


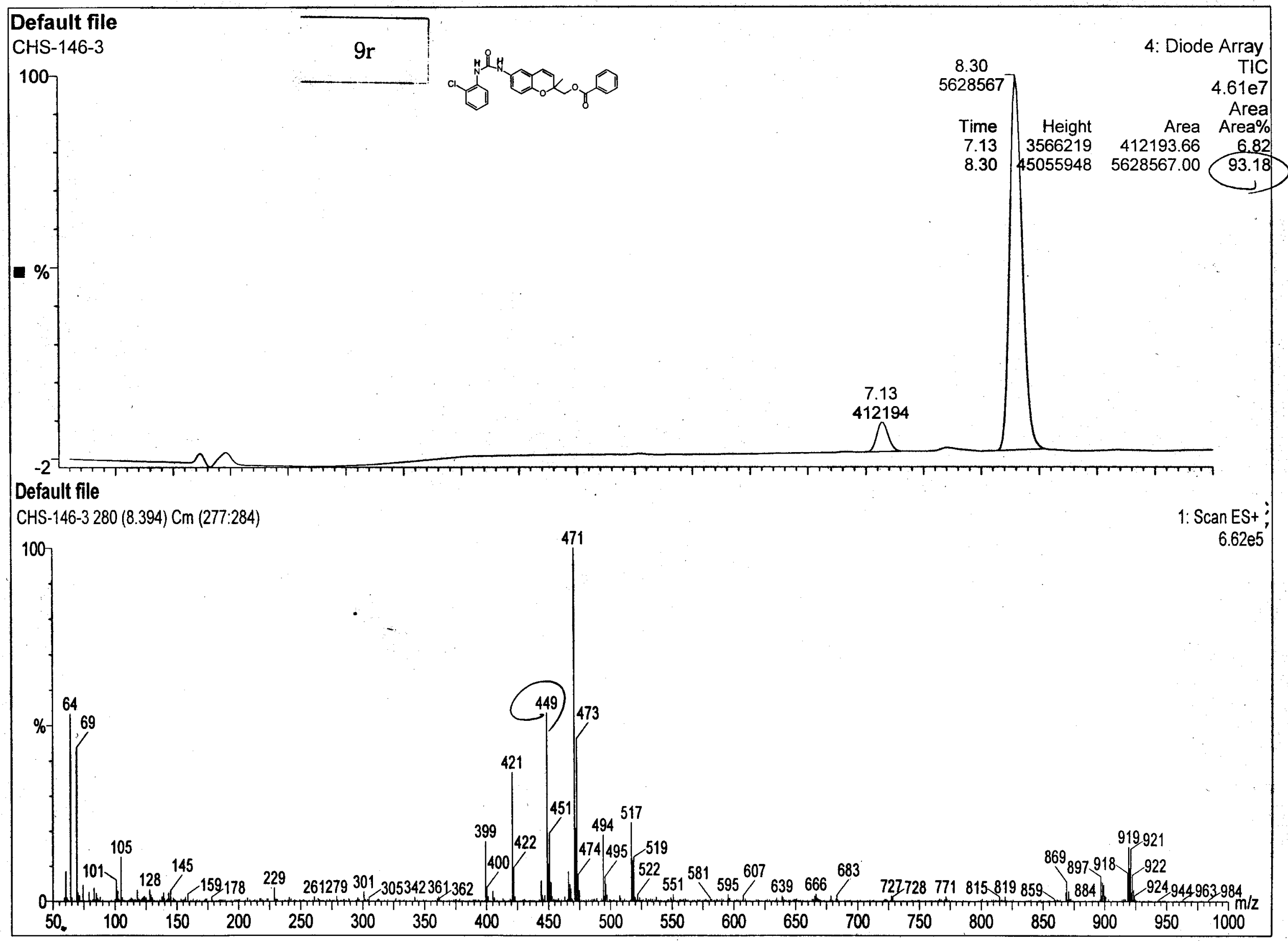




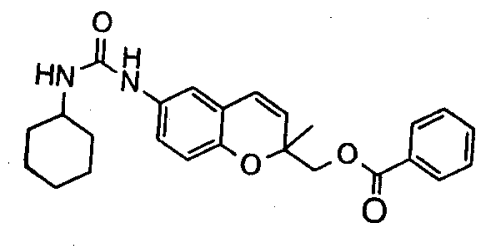

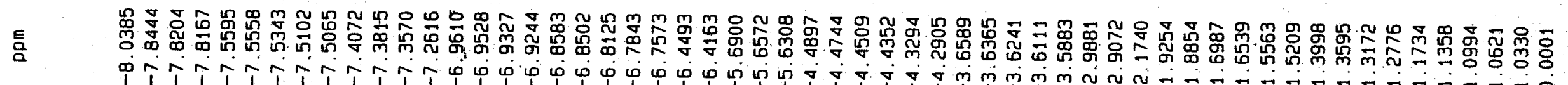
inisiji

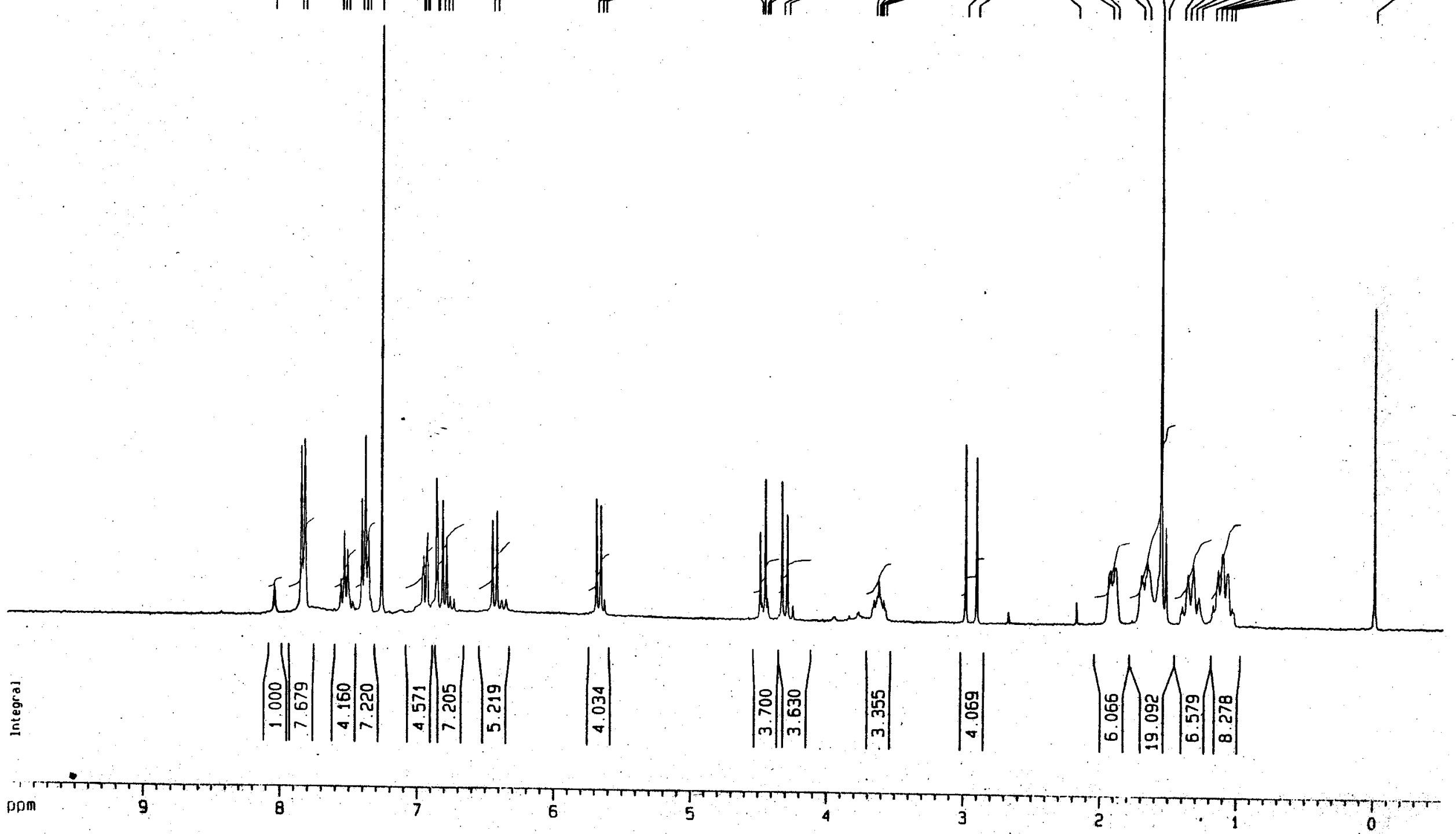




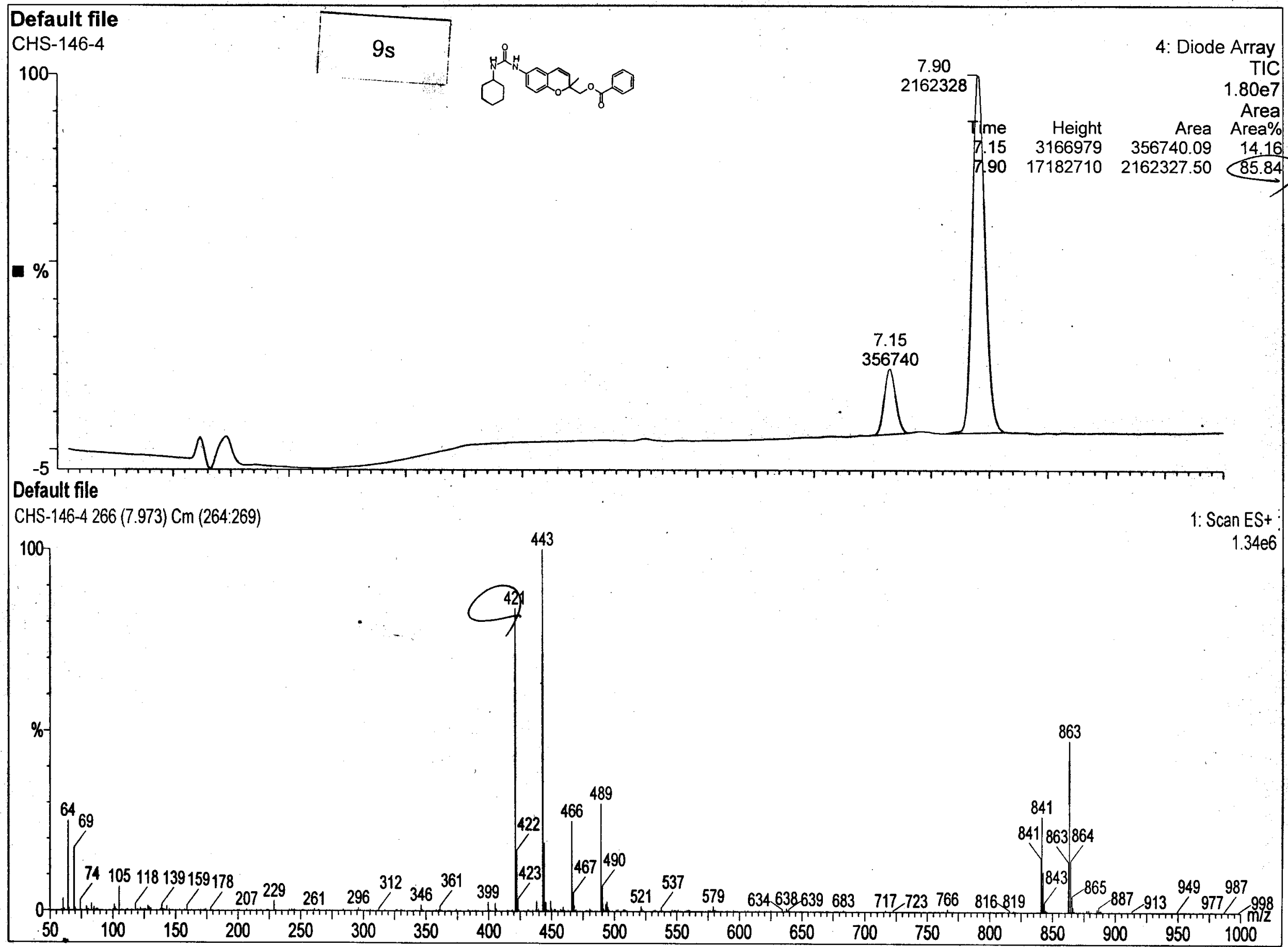




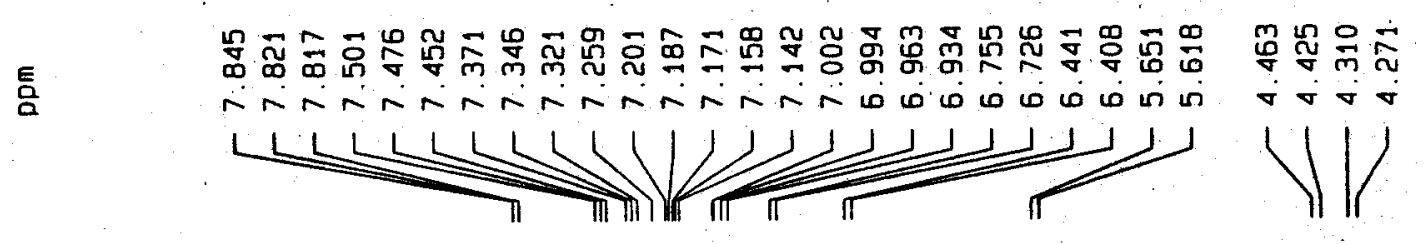

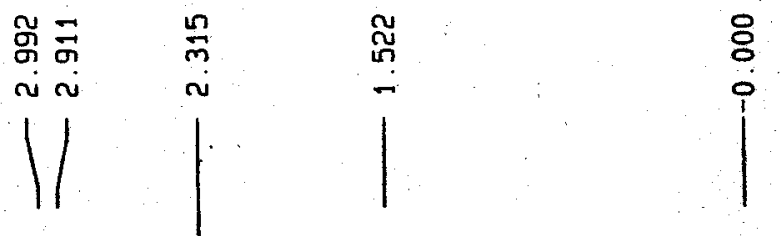

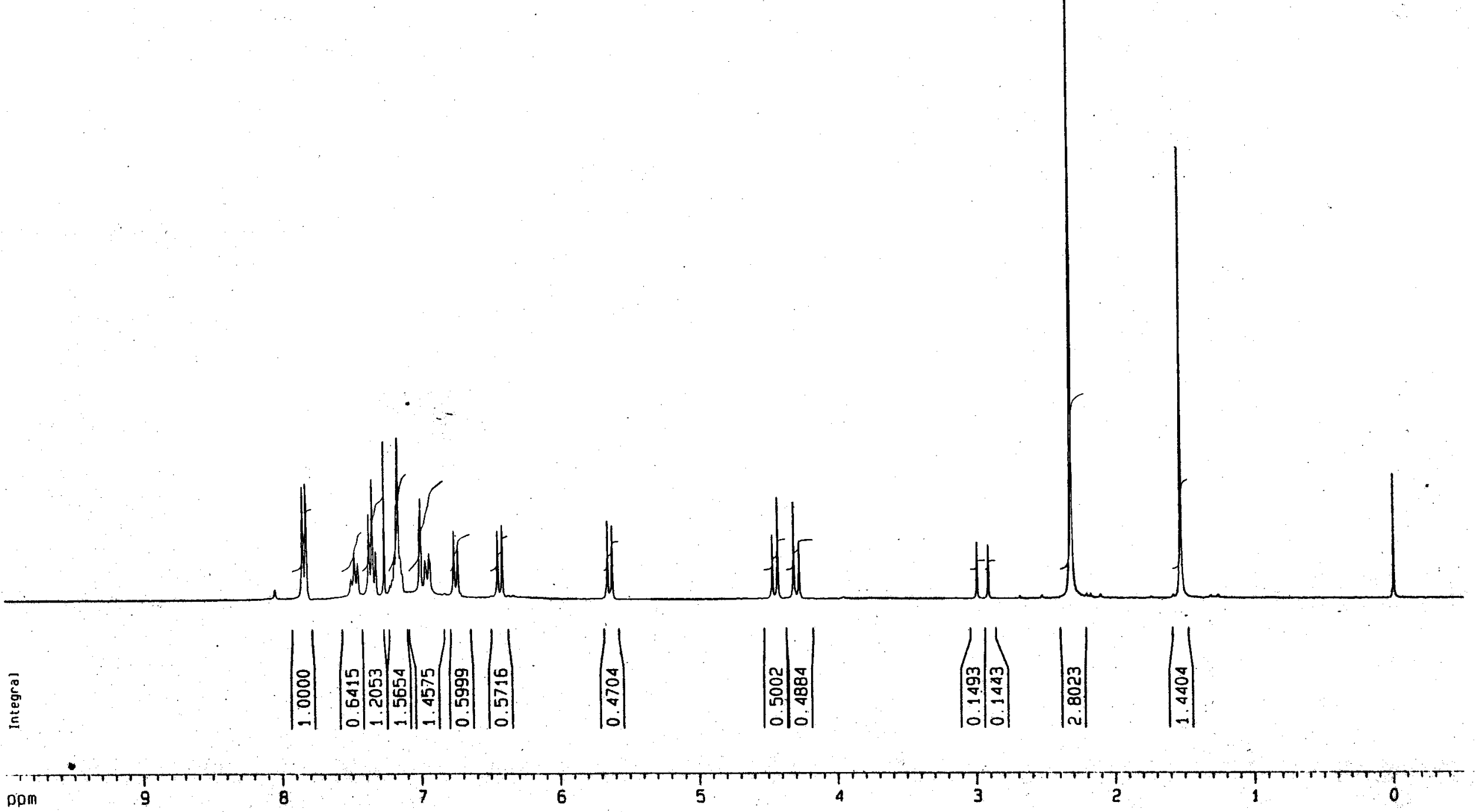




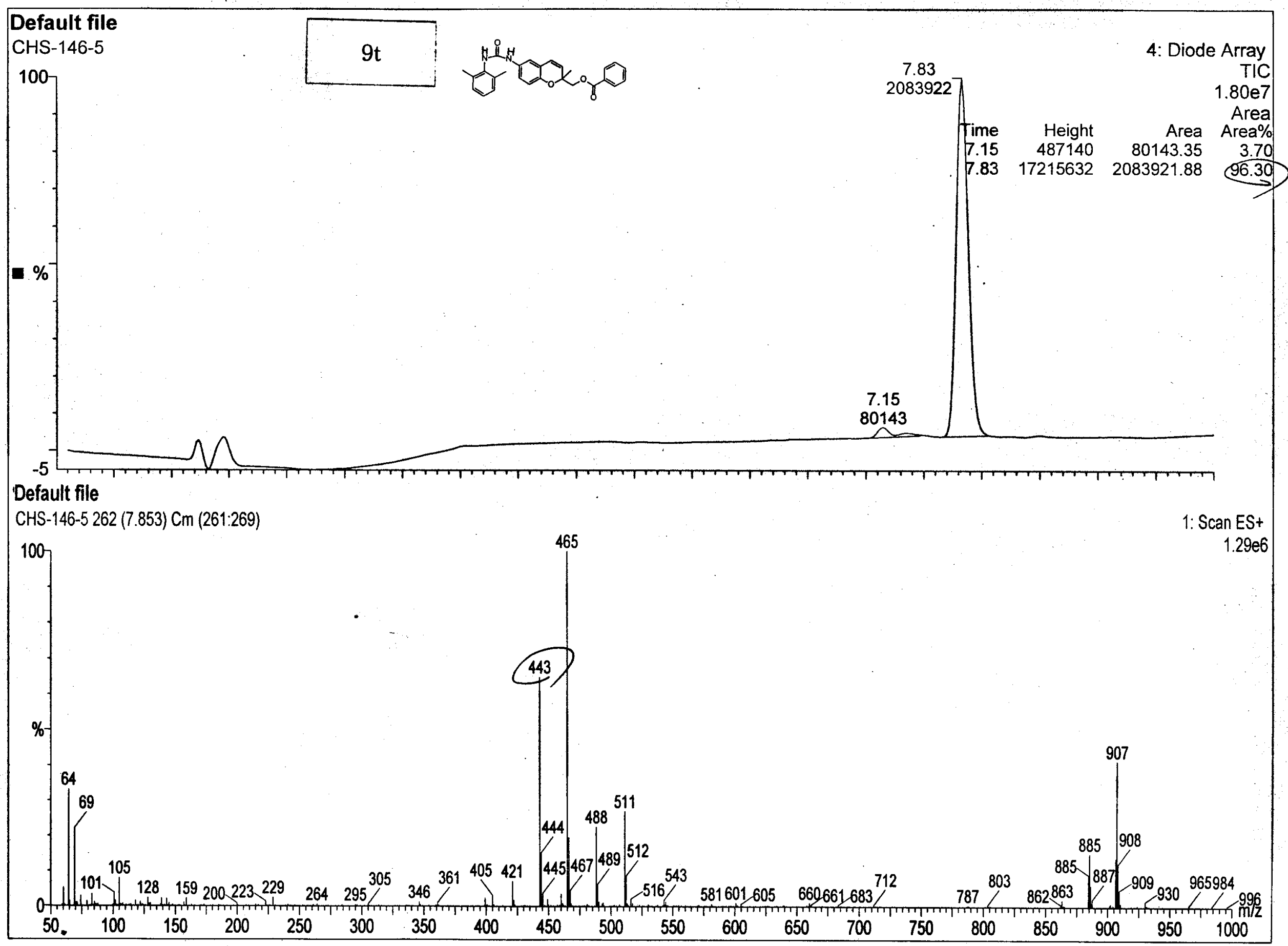


<smiles></smiles>

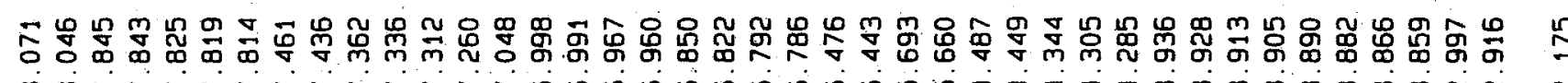
क ôn

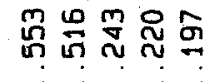

용용 $\longrightarrow$
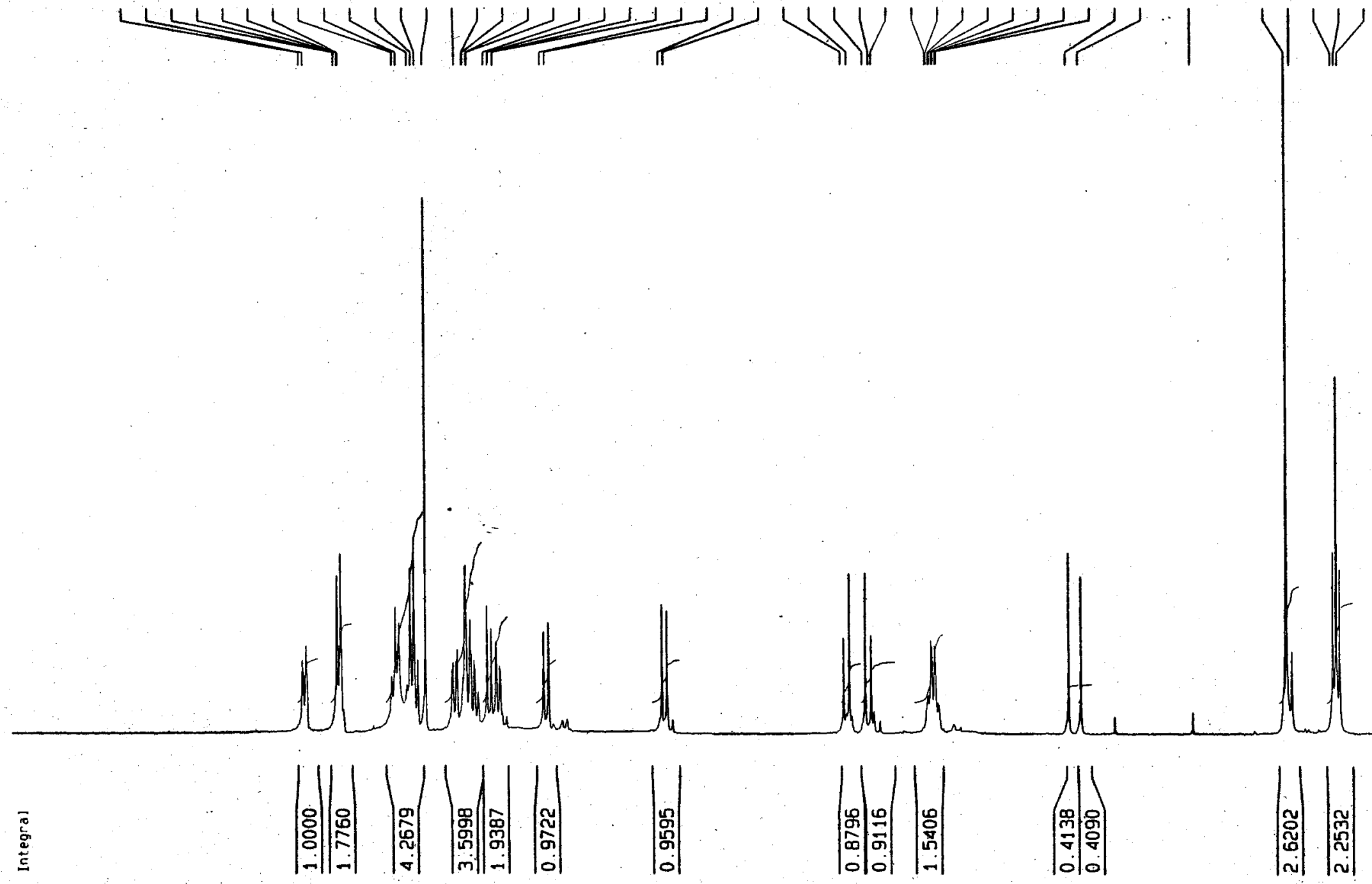


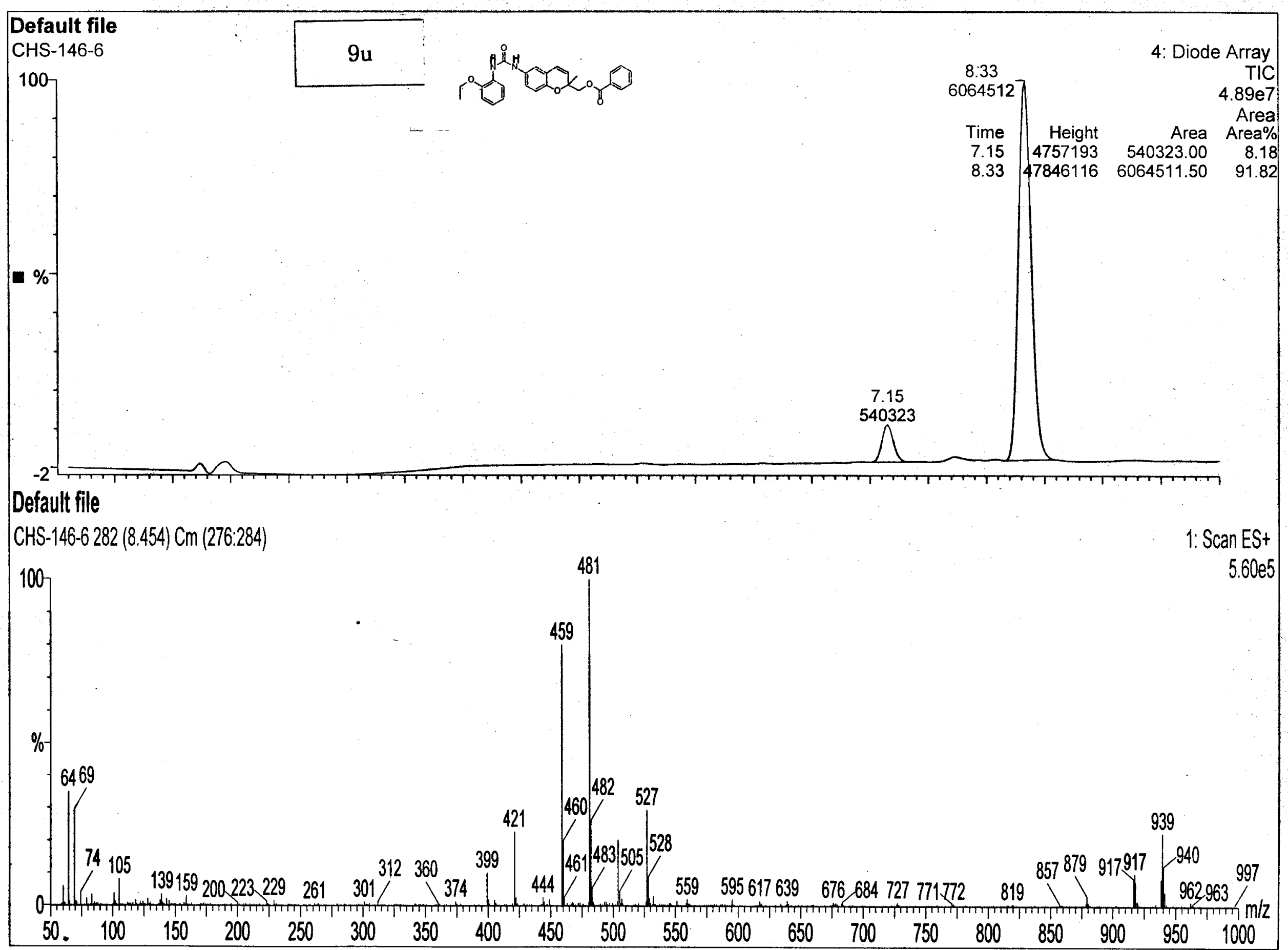




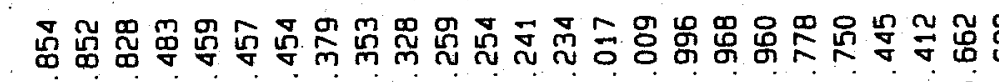

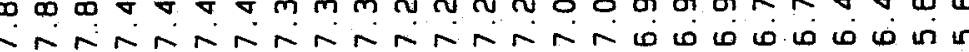
$\longrightarrow$

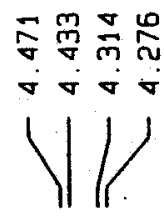

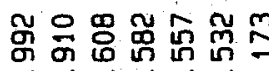

ง
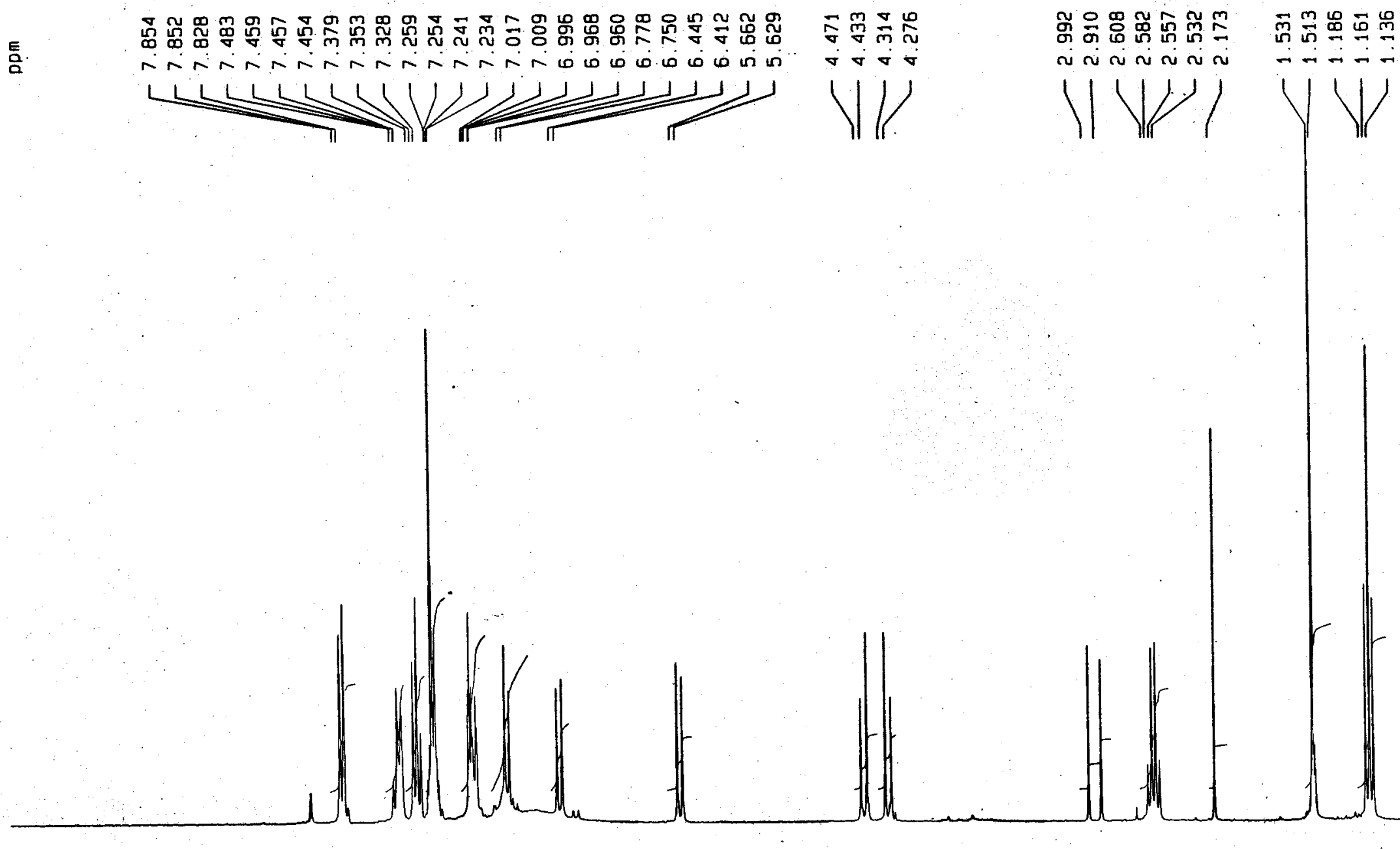

$\underbrace{n+4}$

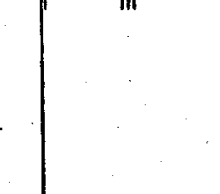

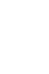



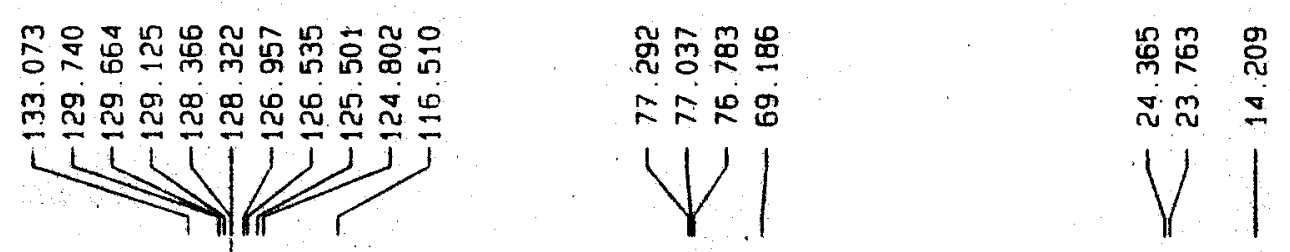

Current Data Parameters

NAME

PROCNO

$=2$ - Acquisition Parameters

Jate $\quad 20050714$

$\begin{array}{ll}\text { TIme } & 12.24 \\ \text { INSTRUM } & \text { 5Dect }\end{array}$

TROBHO $5 \mathrm{~mm}$ DUL $13 C-1$

2ROBRO
دULPROG

65536
SOL VENT COC I3

IS

5WH 35211 ? ?

=IDAES $\quad 0.537281 \mathrm{~Hz}$

$40 \quad 09306754$ SEt

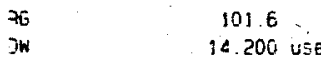

14.200 use:
6.00 use

297 ?

$2.00000000 \mathrm{sec}$

103999998 sec

$189999998 \mathrm{sec}$

$\begin{array}{ll}\text { MCREST } & 0.00000000 \mathrm{SEC} \\ \text { YCWRK } & 0.01500000 \mathrm{SEC}\end{array}$

$==x=x=$ CHANNEL if $= \pm= \pm= \pm$

$\begin{array}{lr}\text { WuC } & 13 C \\ J_{1} & 7.50 \text { usec } \\ =11 & -1.00 \mathrm{oB}\end{array}$

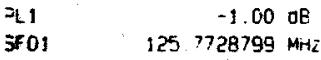

$===z=x$ CHANNEL f $2== \pm==3$

CPOPAG W Wajtz 16

$\begin{array}{ll}\text { VUC2 } & 1 \mathrm{H} \\ \text { JCPDE } & \quad 80.00 \text { use }\end{array}$

गL.

$-1.40 \mathrm{~dB}$

$x 13 \quad 25.00 \mathrm{~dB}$
$x .13$

25 - Processing parameters

SI Processing parameters

SF $\quad 125.7577911 \mathrm{MHZ}$

SSB $\quad$ EM

$\begin{array}{cc}-B & 0 \\ G B & 0\end{array}$

1.40

10 Nmp plot parameters

$\begin{array}{ll}\mathrm{CX} & 20.00 \mathrm{~cm} \\ \mathrm{Cr} & 12.50 \mathrm{~cm}\end{array}$

cr

$\quad 200.000 \mathrm{ppm}$
$=1$$\quad \begin{array}{ll}25151.56 \mathrm{~Hz} \\ -1 \mathrm{P}\end{array}$

द2P
$5 \mathrm{P}$
$-20.013 \mathrm{ppm}$

F2 $\quad-2516.83 \mathrm{~Hz}$

PPMCM $\quad 11.00067 \mathrm{pDm} / \mathrm{Cm}$ 


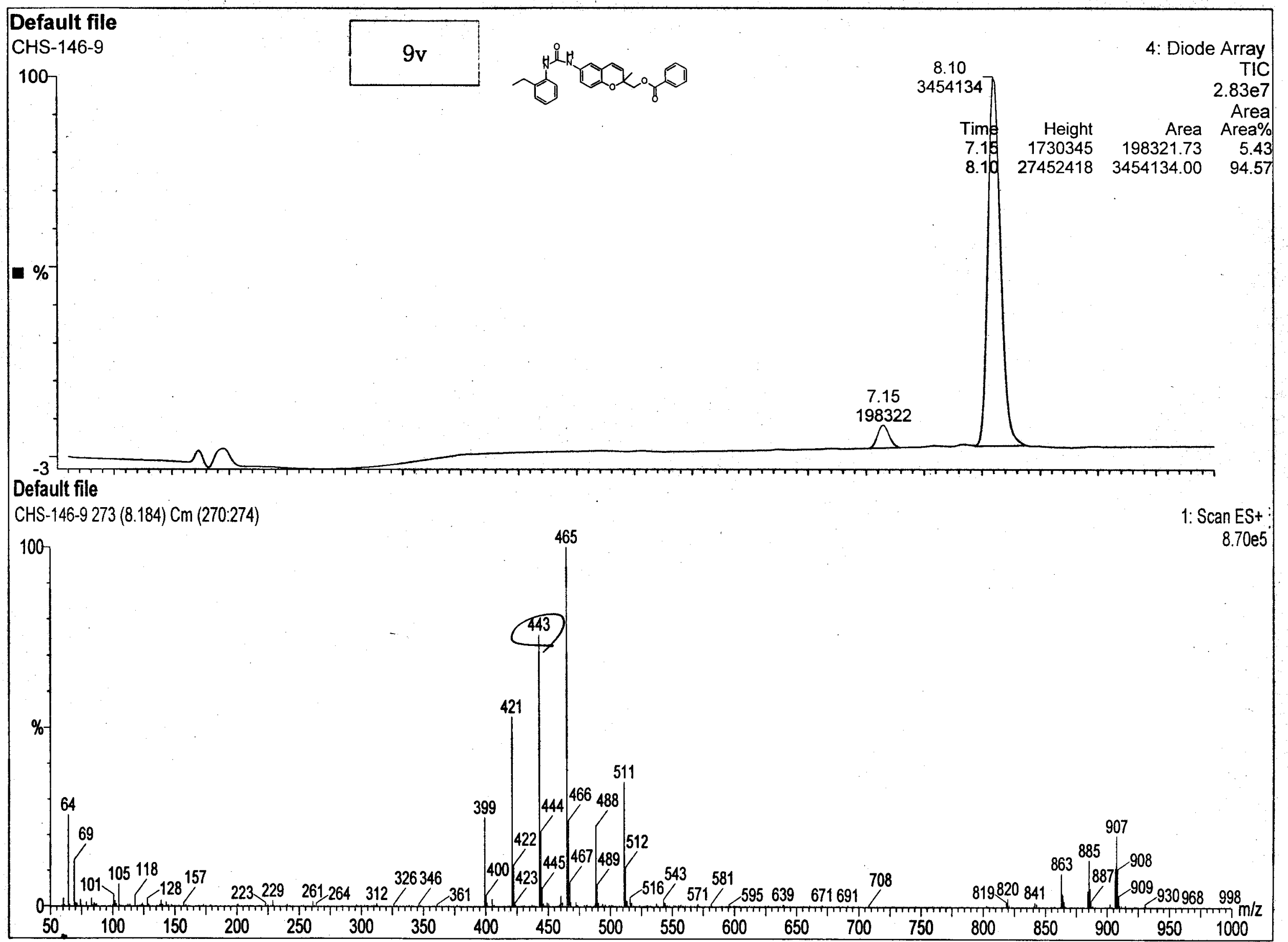




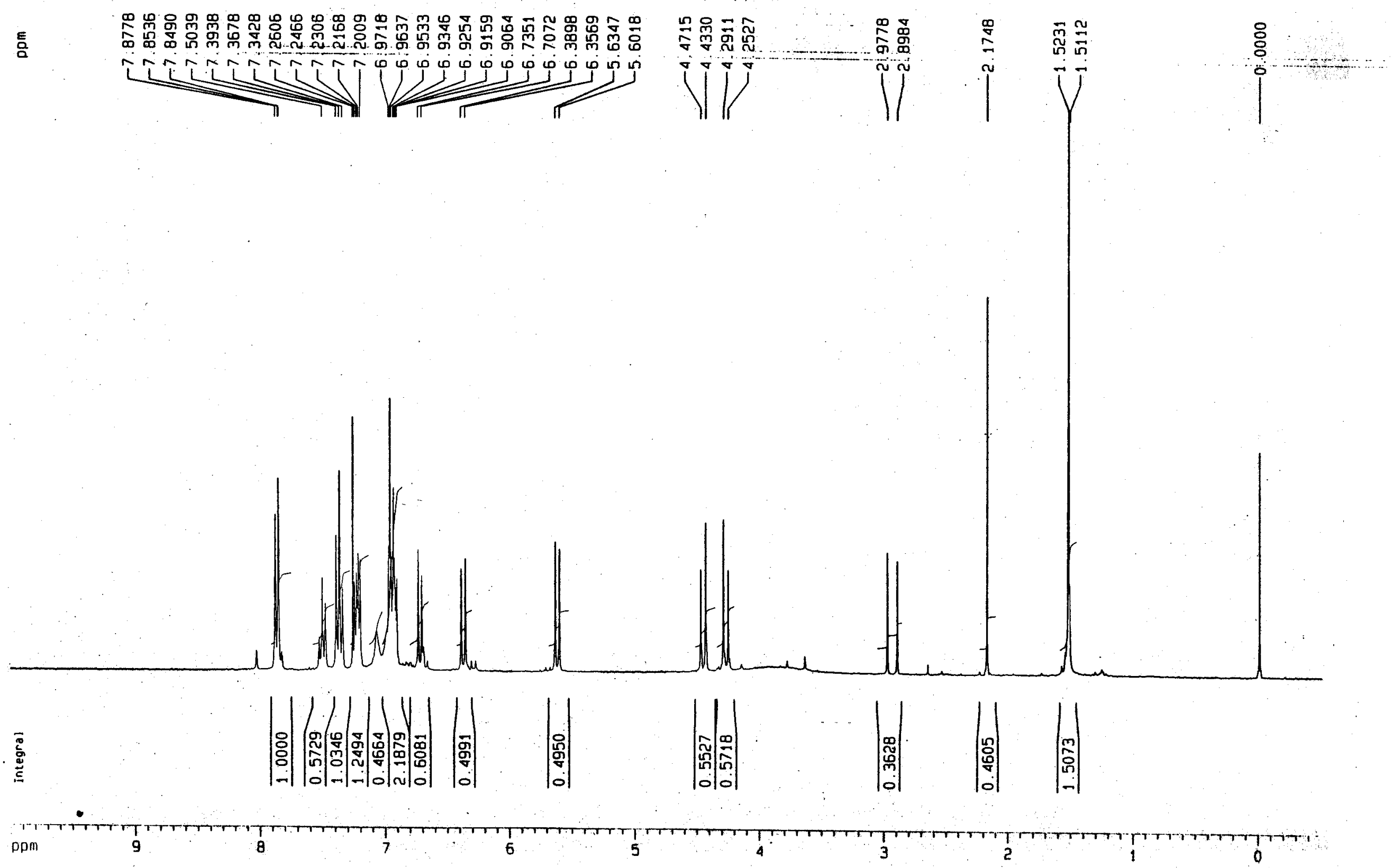




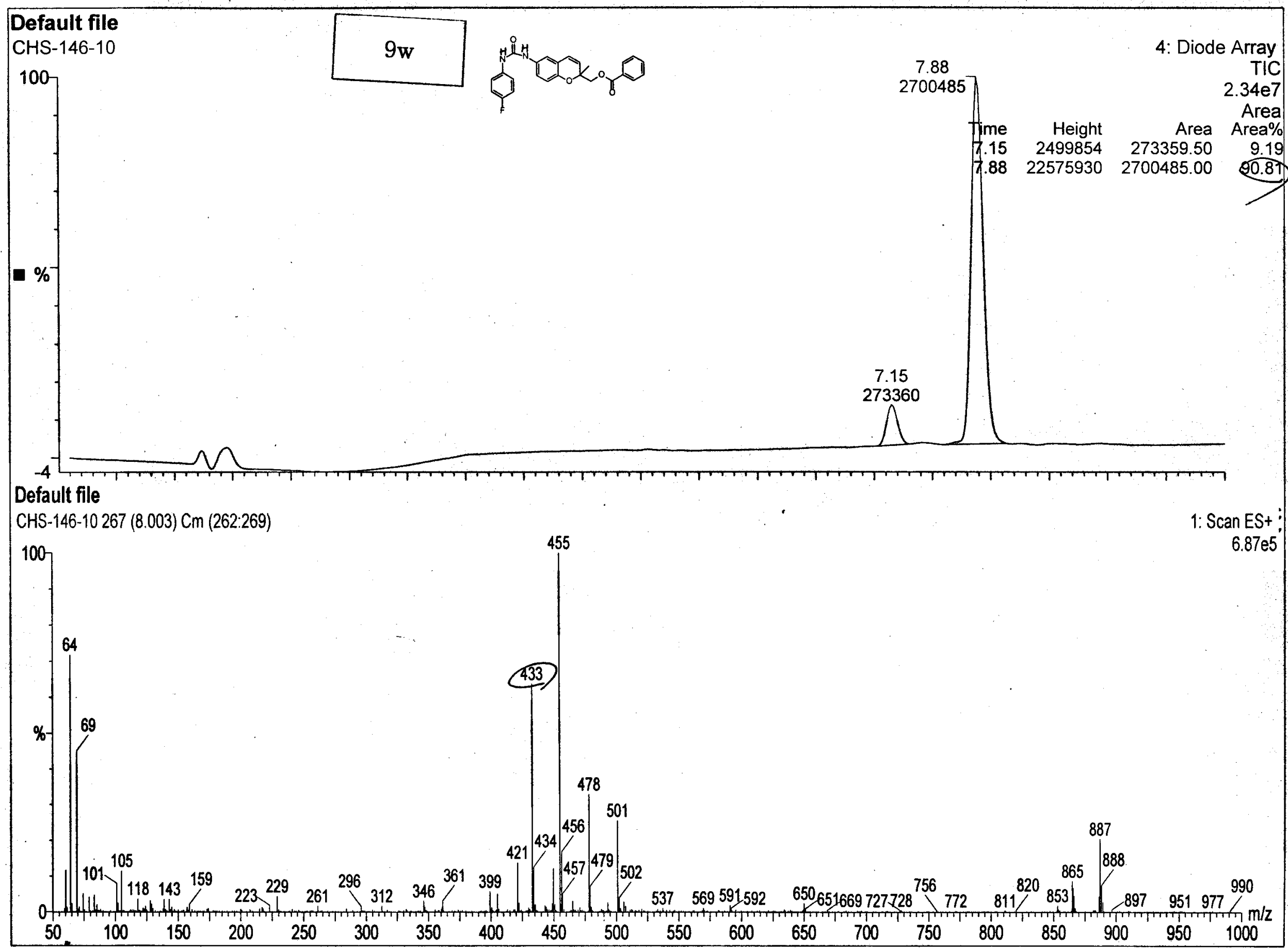



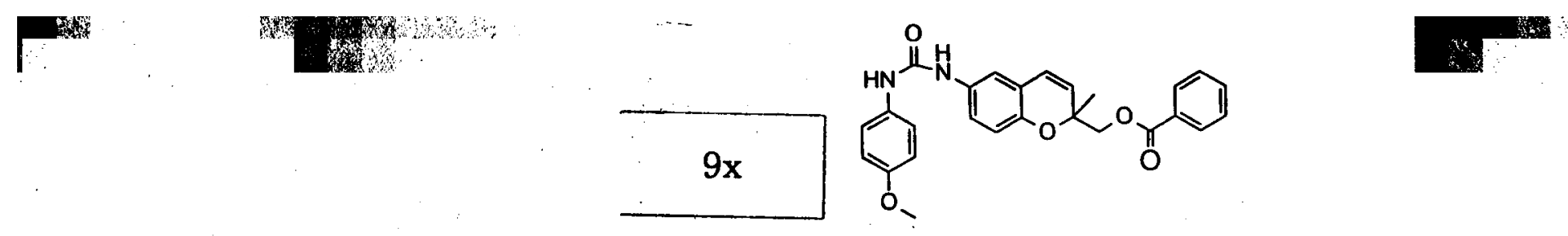

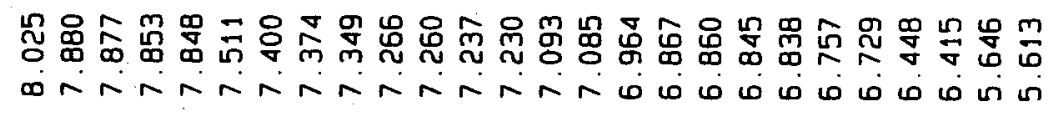
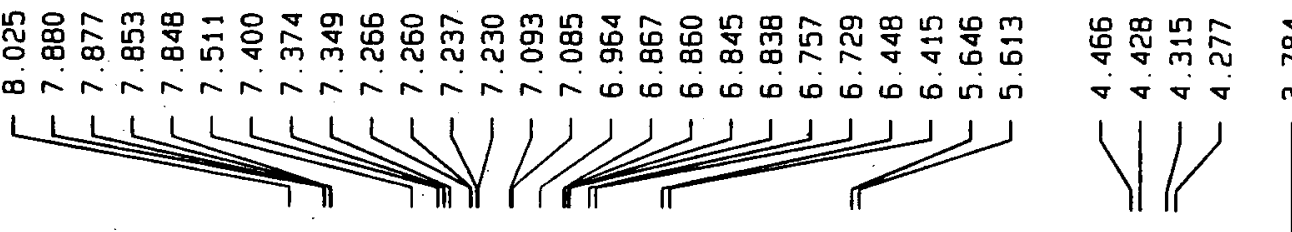

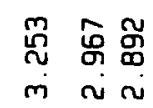

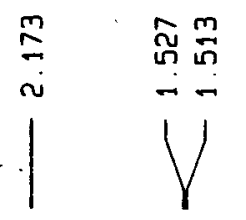

흥

11

in

$i$
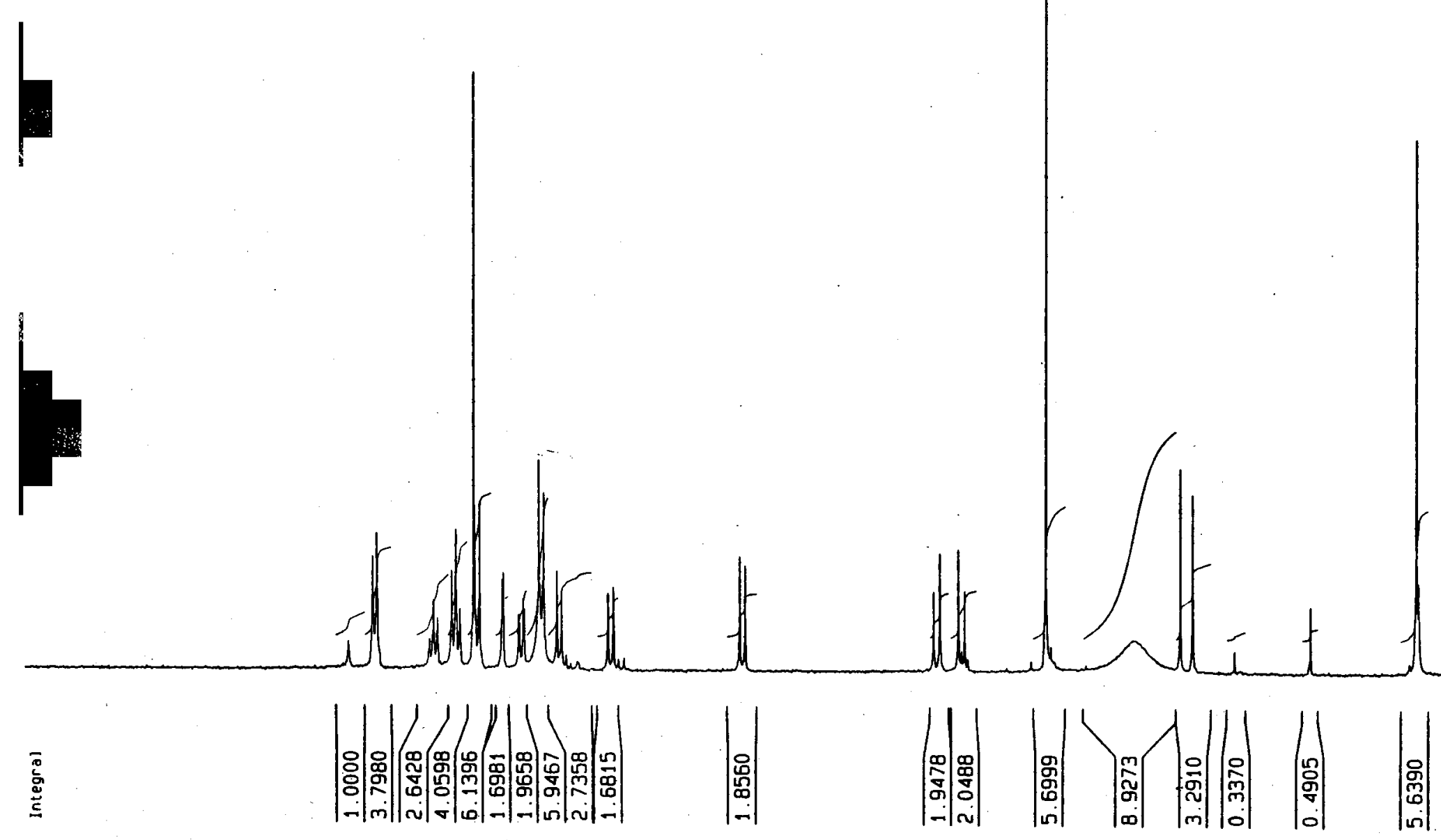

ppm

9

8

4 


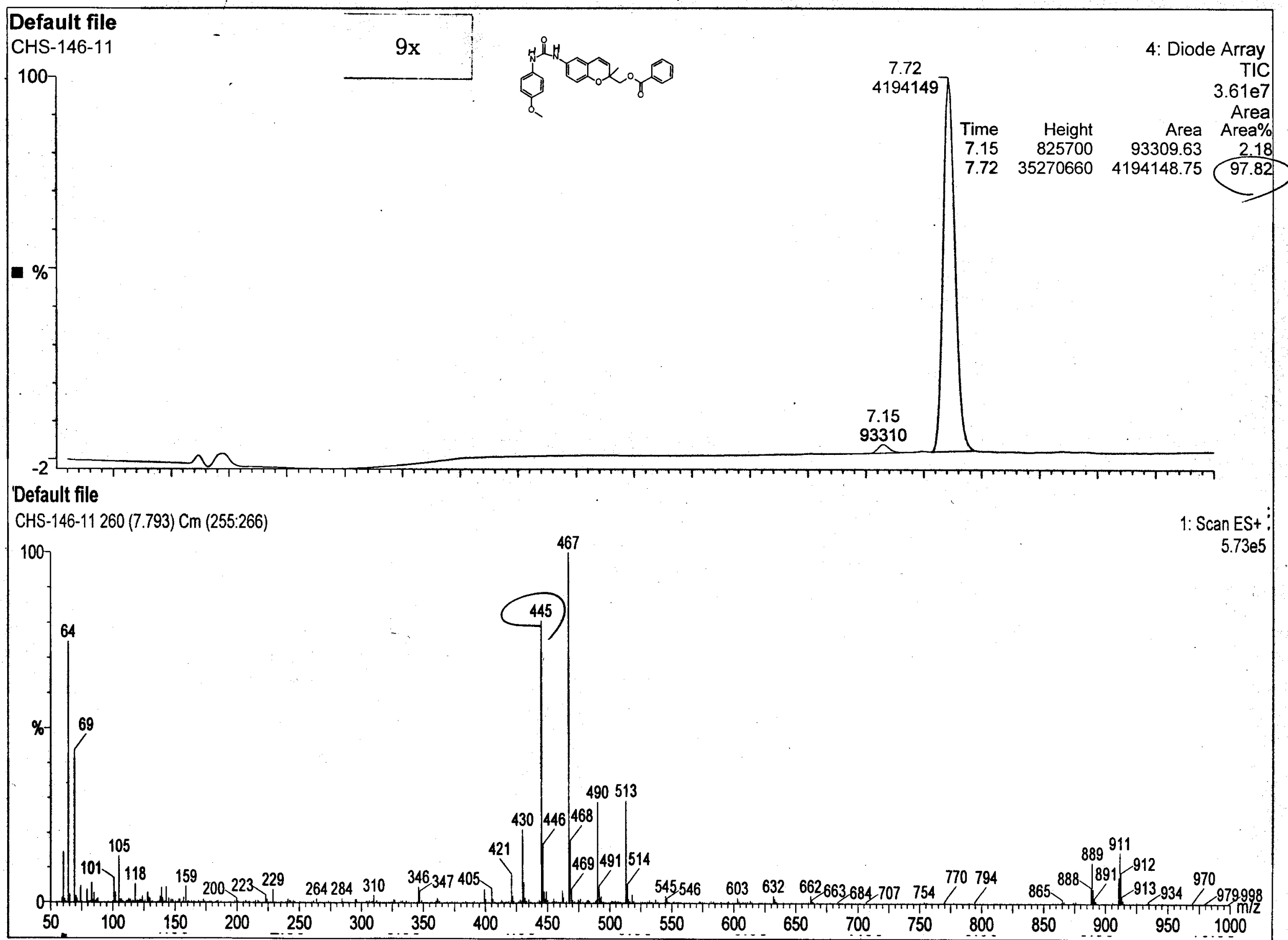


$9 \mathrm{y}$

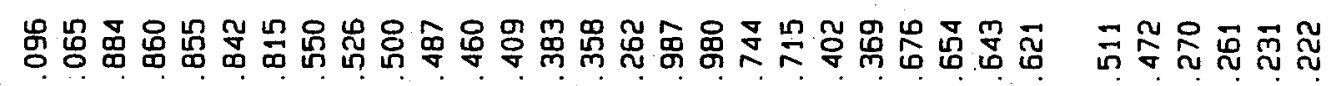

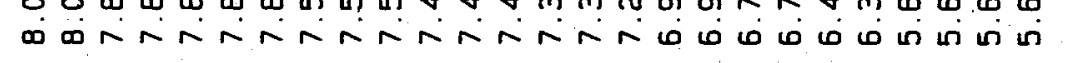
(1)

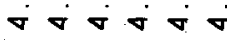
yV
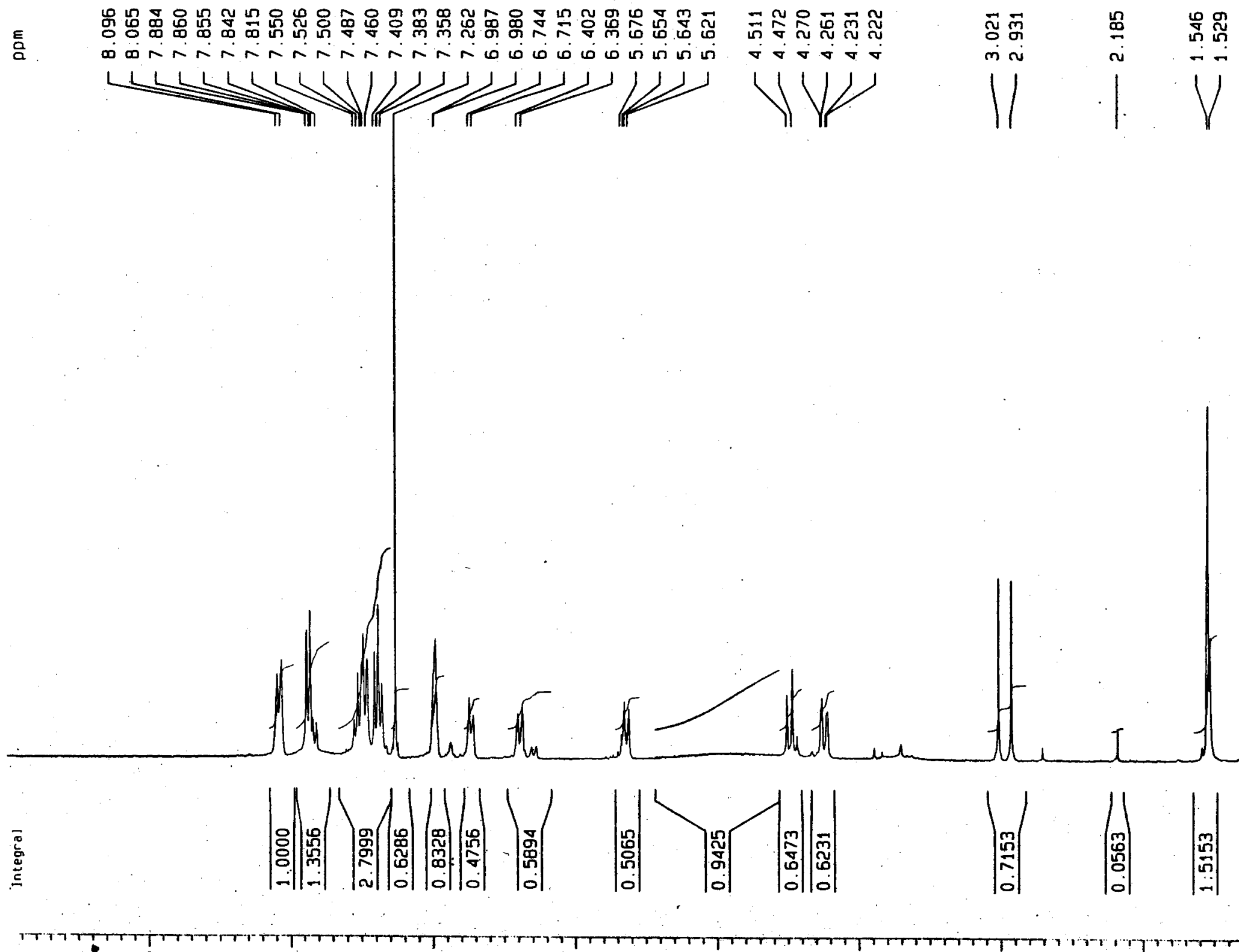


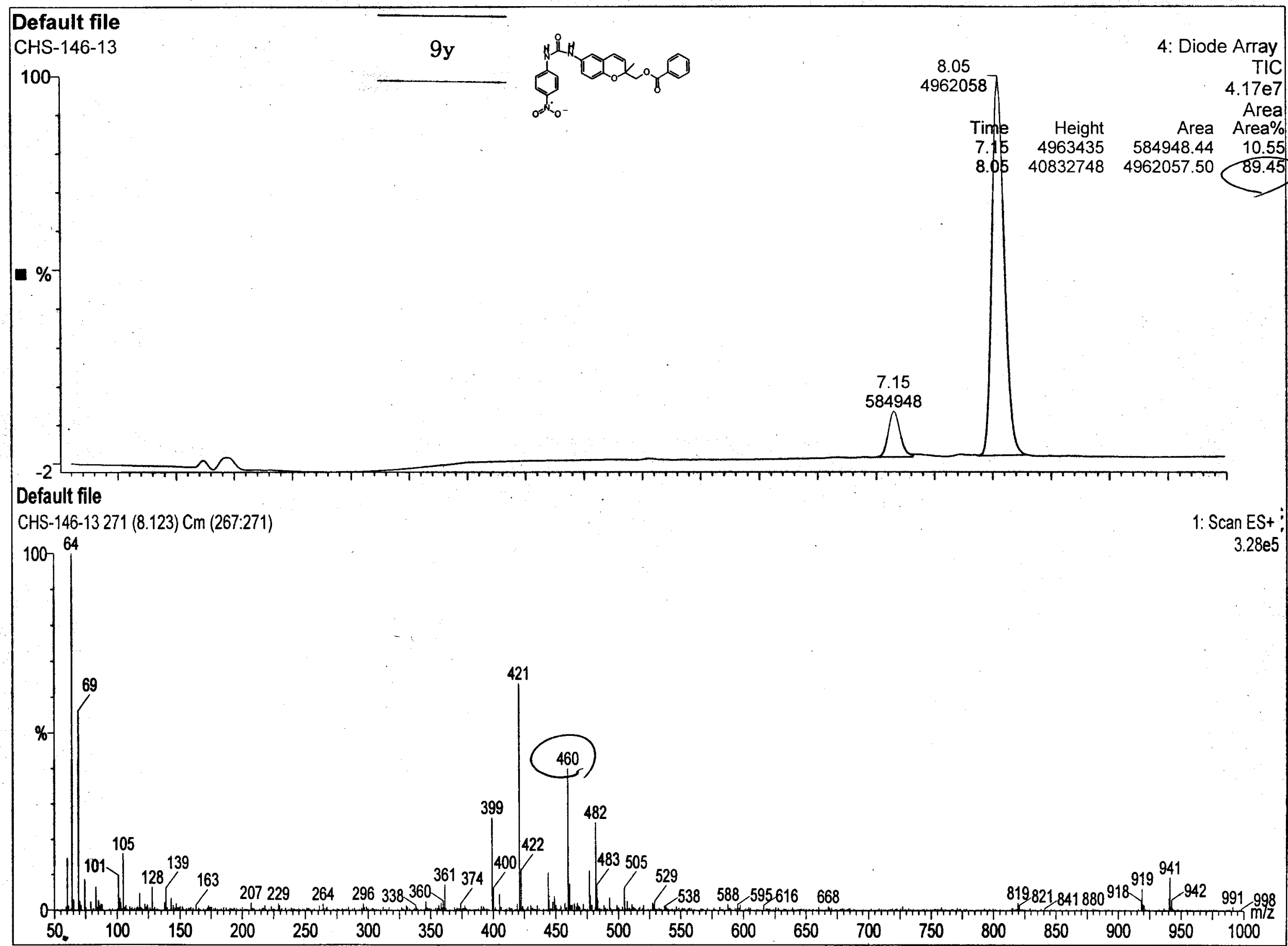




\section{$12 \mathrm{a}$}

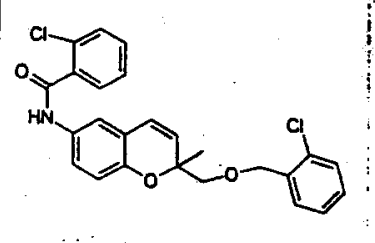

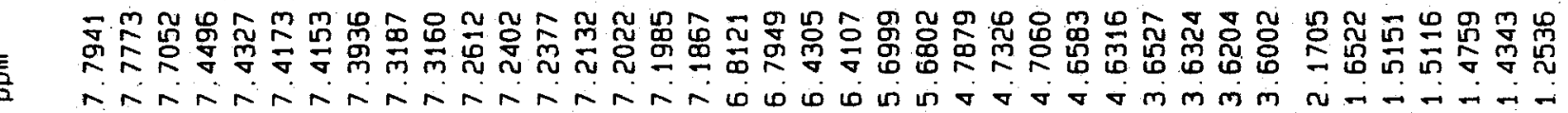

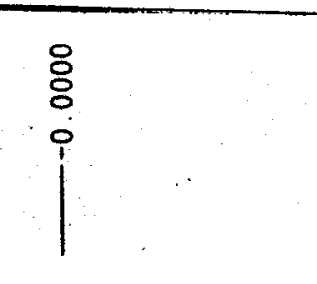

Current Data Parameters

Name chs-b56-4

EXPNO

PROCNO

1

F2 - Acquisition Parameters

Date_ 20040908

Time 20.44

$5 \mathrm{~mm}$ DUL $13 \mathrm{Cpect}$

PULPROG

SOL VENT

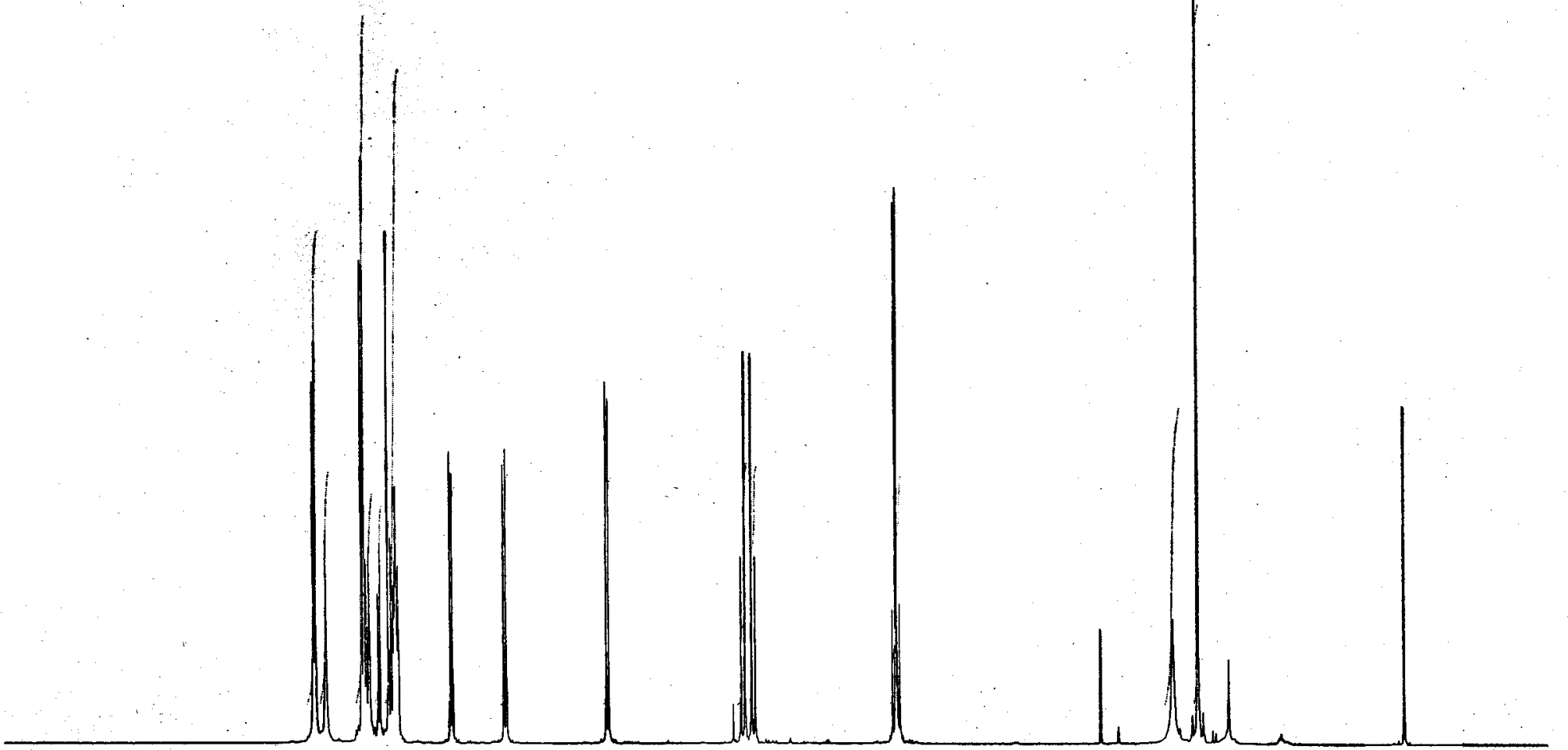

NS

$\begin{array}{lr}\text { DS } & 0 \\ \text { SWH } & 7507.507 \mathrm{~Hz} \\ \text { FIOES } & 0.114555 \mathrm{~Hz}\end{array}$

FIDRES $\quad 0.114555 \mathrm{~Hz}$

AQ

4. 3648143 sec

RG

DW

DE

$\begin{array}{rr}\text { TE } & 0.0 \mathrm{~K} \\ \text { D1 } & 1.00000000 \mathrm{sec}\end{array}$

MCWAK $\quad 0.01500000$ sec

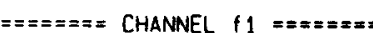

NUCI

$\begin{array}{ll}\text { NUC1 } & 1 \mathrm{H} \\ \mathrm{P}_{1} & 10.00 \text { uset }\end{array}$

PL1 $\quad-2.00 \mathrm{~dB}$

SF01 $500.1332508 \mathrm{MHZ}$

F2 - Processing parameters

SI 32768

SF $\quad 500.1300130 \mathrm{MHz}$

WOW $5 S B \quad$ EM

$5 S B$

LB

$0.30 \mathrm{~Hz}$

1.00

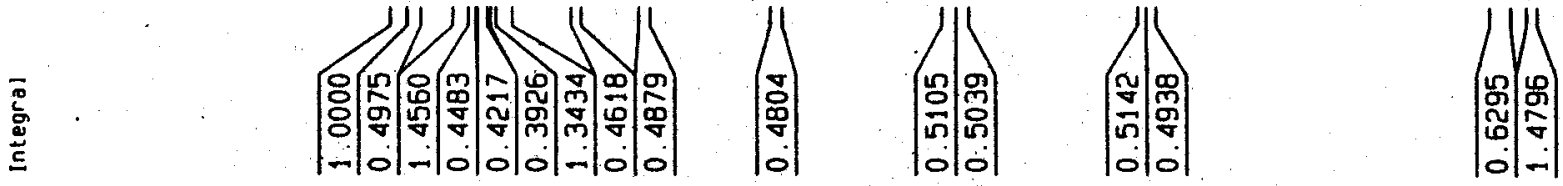

10 NMA plot parameters

CX $\quad 20.00 \mathrm{~cm}$

$\mathrm{CY} \quad 25.00 \mathrm{~cm}$

F1P $\quad 10.000 \mathrm{pom}$

$\mathrm{Fr}^{2} \quad 5001.30 \mathrm{~Hz}$

F2P $\quad-1.031 \mathrm{DDm}$

$F 2 \quad-515.55 \mathrm{~Hz}$

$\begin{array}{lr}\text { PPMCM } & 0.55154 \mathrm{ppm} / \mathrm{cm} \\ \mathrm{HZCM} & 275.84250 \mathrm{~Hz} / \mathrm{cm}\end{array}$

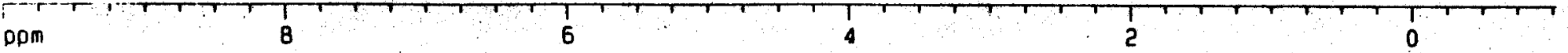




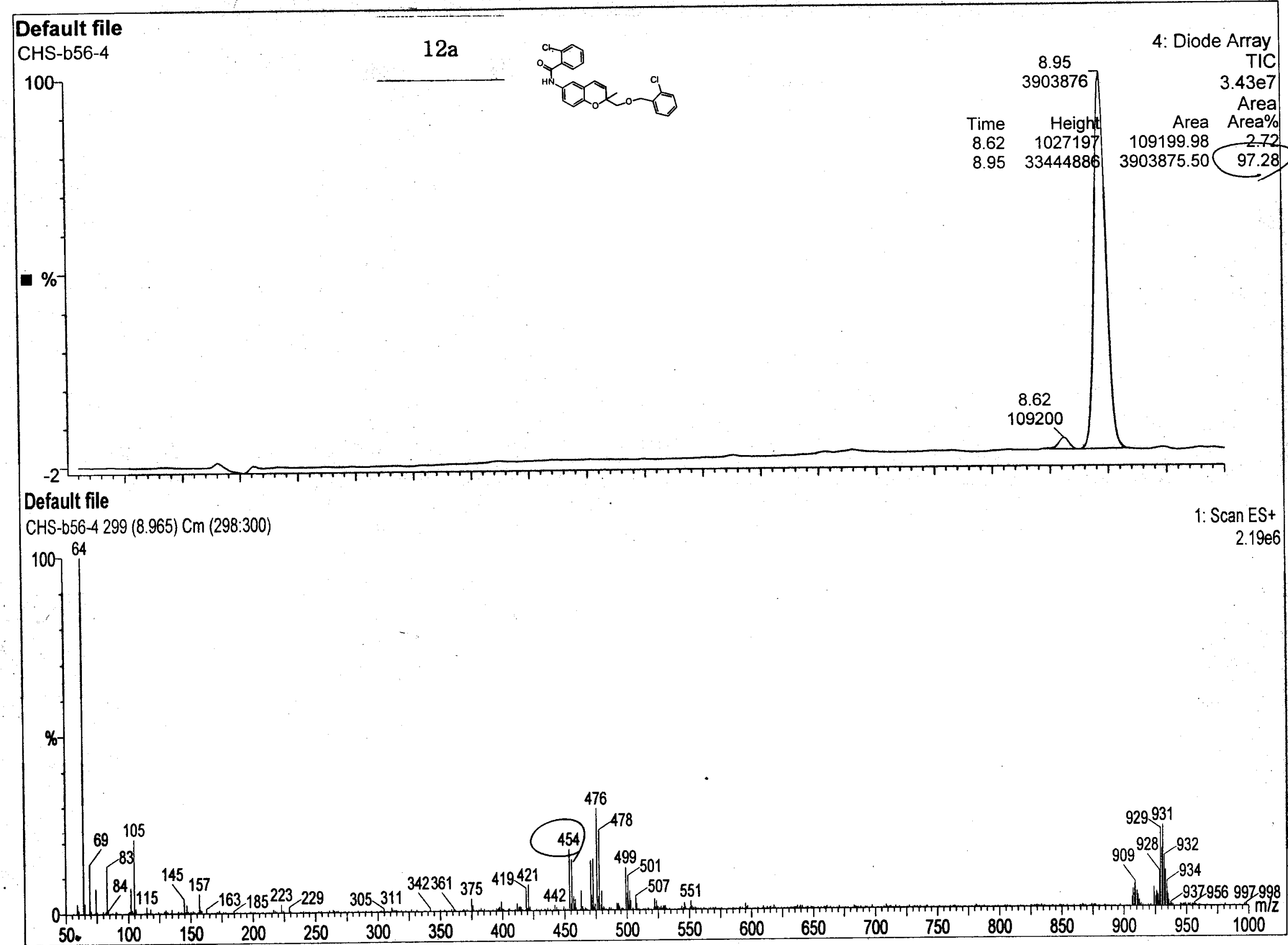




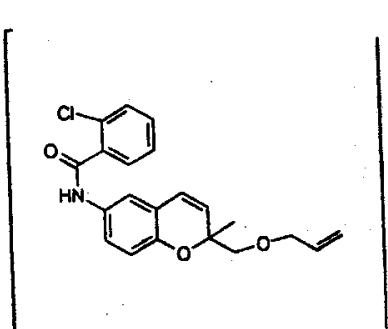

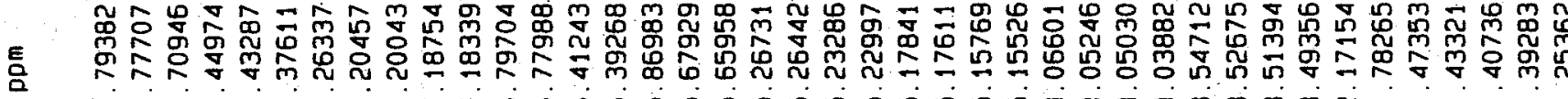

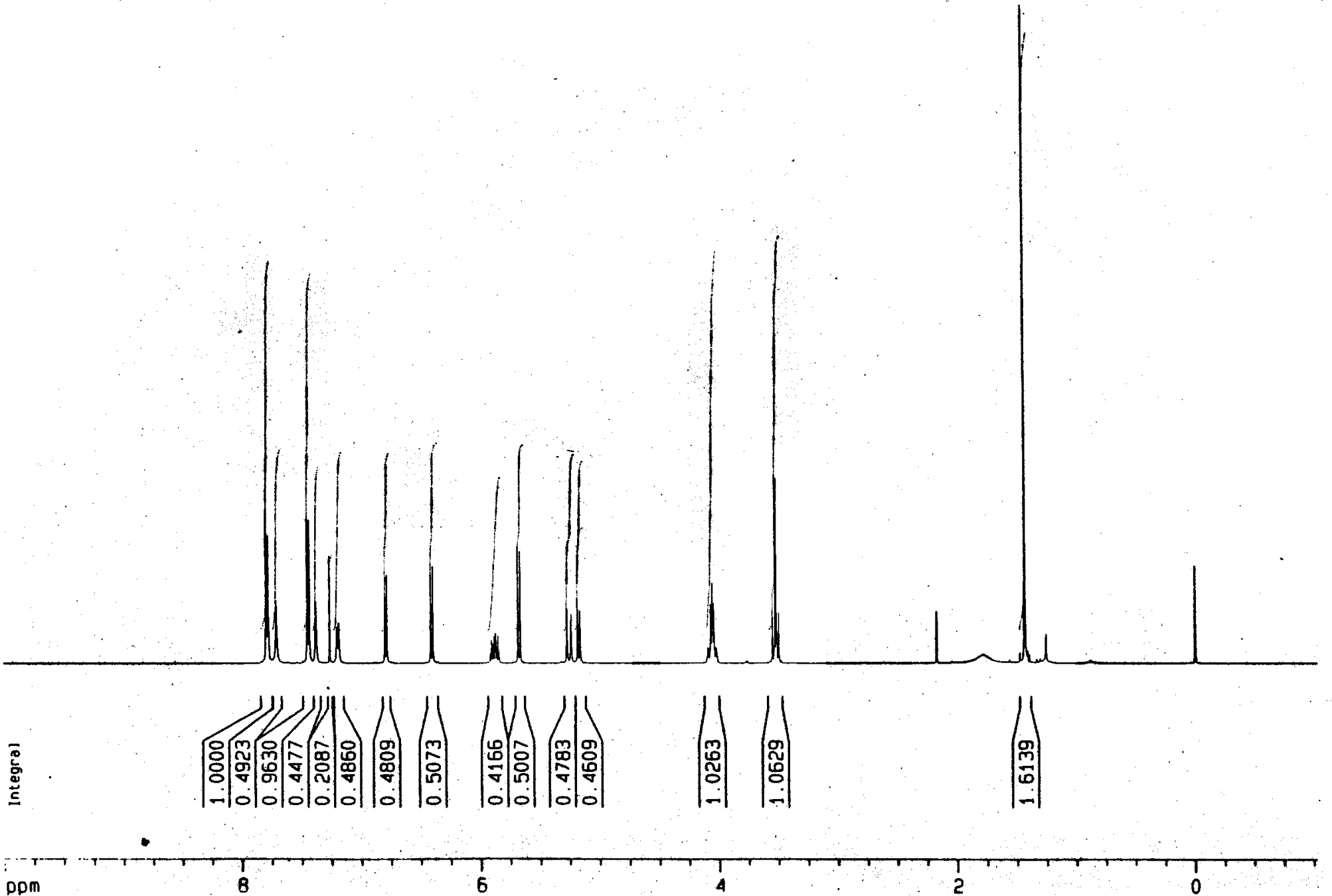

Current Data Parameters EXPNO PROCNO

F2 - Acquisition Parameters

Date _ 20040908

Ijme $\quad 21.23$

INSTRUM spect

PROBHO $5 \mathrm{~mm}$ DUL $13 \mathrm{C}-1$

$13 C-1$
29
65536

TD

SOLVENT COCI3

NS

DS

SWH

AO $\quad 0.114555 \mathrm{~Hz}$

$A Q$

RG

DE

TE

DE

$0.0 \mathrm{~K}$

MCREST $\quad 0.0000000 \mathrm{sec}$

$0.00000000 \mathrm{sec}$

$== \pm= \pm=$ CHANNEL $f 11== \pm= \pm=0$ NUC

SFO1 500.1332508 Mtz

F2 - Processing parameters

SI $\quad 32768$

SF $\quad 500.1300119 \mathrm{MHz}$

WOW EM

$\begin{array}{lr}\text { SSB } & 0 \\ \text { LB } & 0.30 \mathrm{H}\end{array}$

$\begin{array}{lr}\text { GB } & 0 \\ \text { PC } & 1.00\end{array}$

10 NMR plot parameters

CX $20.00 \mathrm{~cm}$

$\mathrm{CY} \quad 10.00 \mathrm{~cm}$

F1P $\quad 10.000 \mathrm{ppm}$

F1 $5001.30 \mathrm{~Hz}$

F2P $\quad-1.031 \mathrm{ppm}$

F2 20.5

$\begin{array}{lr}\text { PPMCM } & 0.55157 \mathrm{DDm} / \mathrm{Cm} \\ \mathrm{HZCM} & 275.85828 \mathrm{~Hz} / \mathrm{Cm}\end{array}$ 


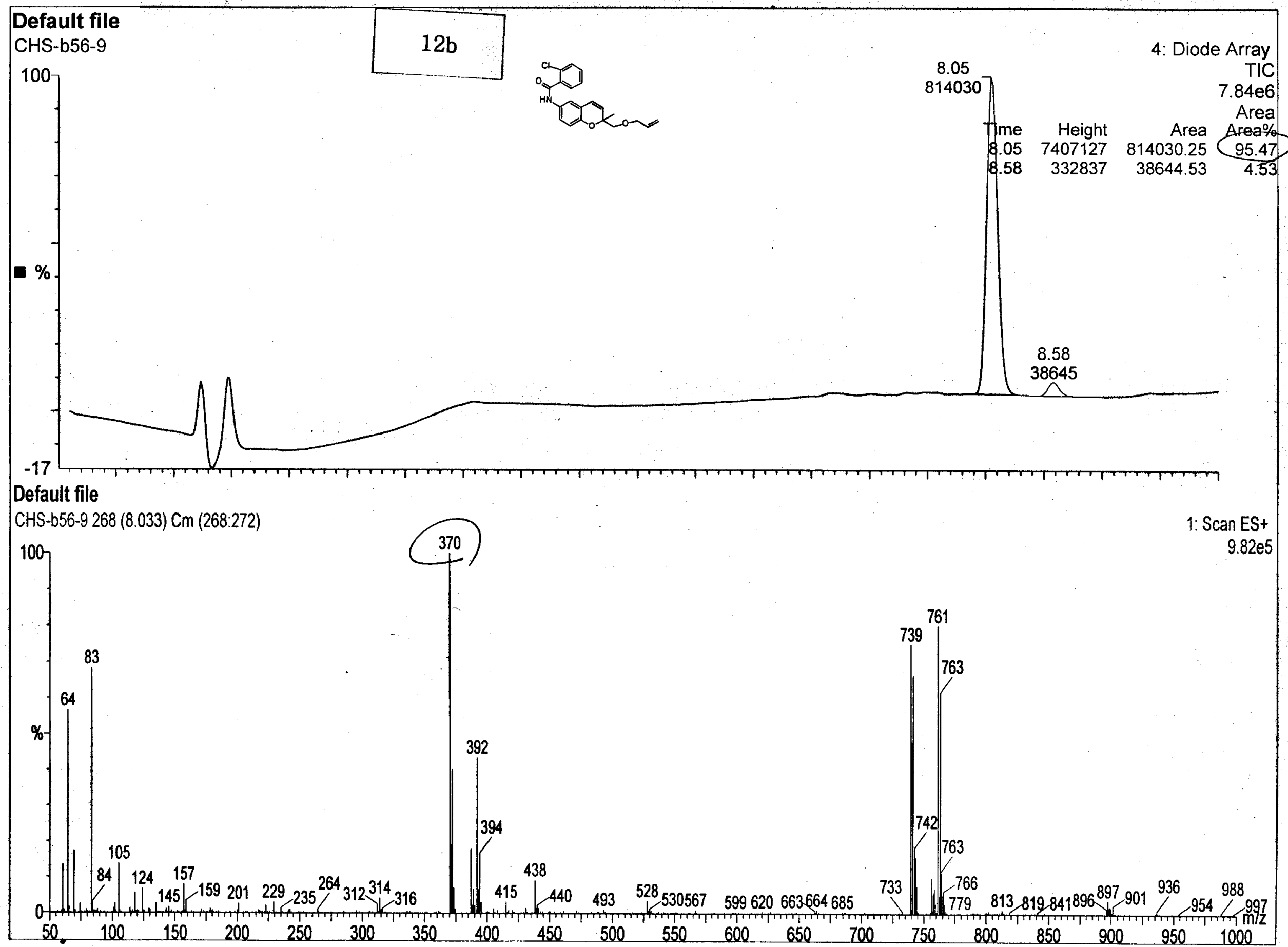




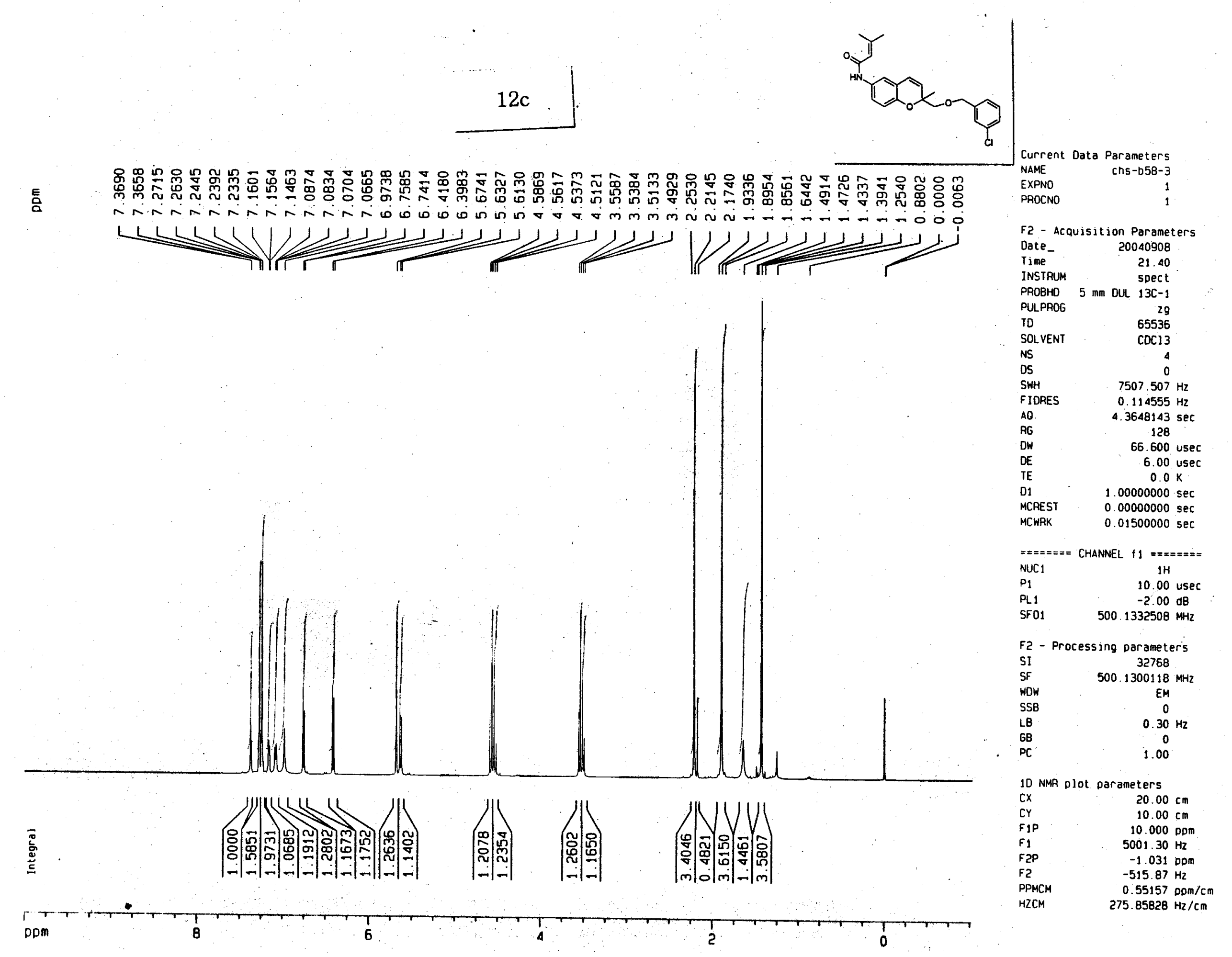




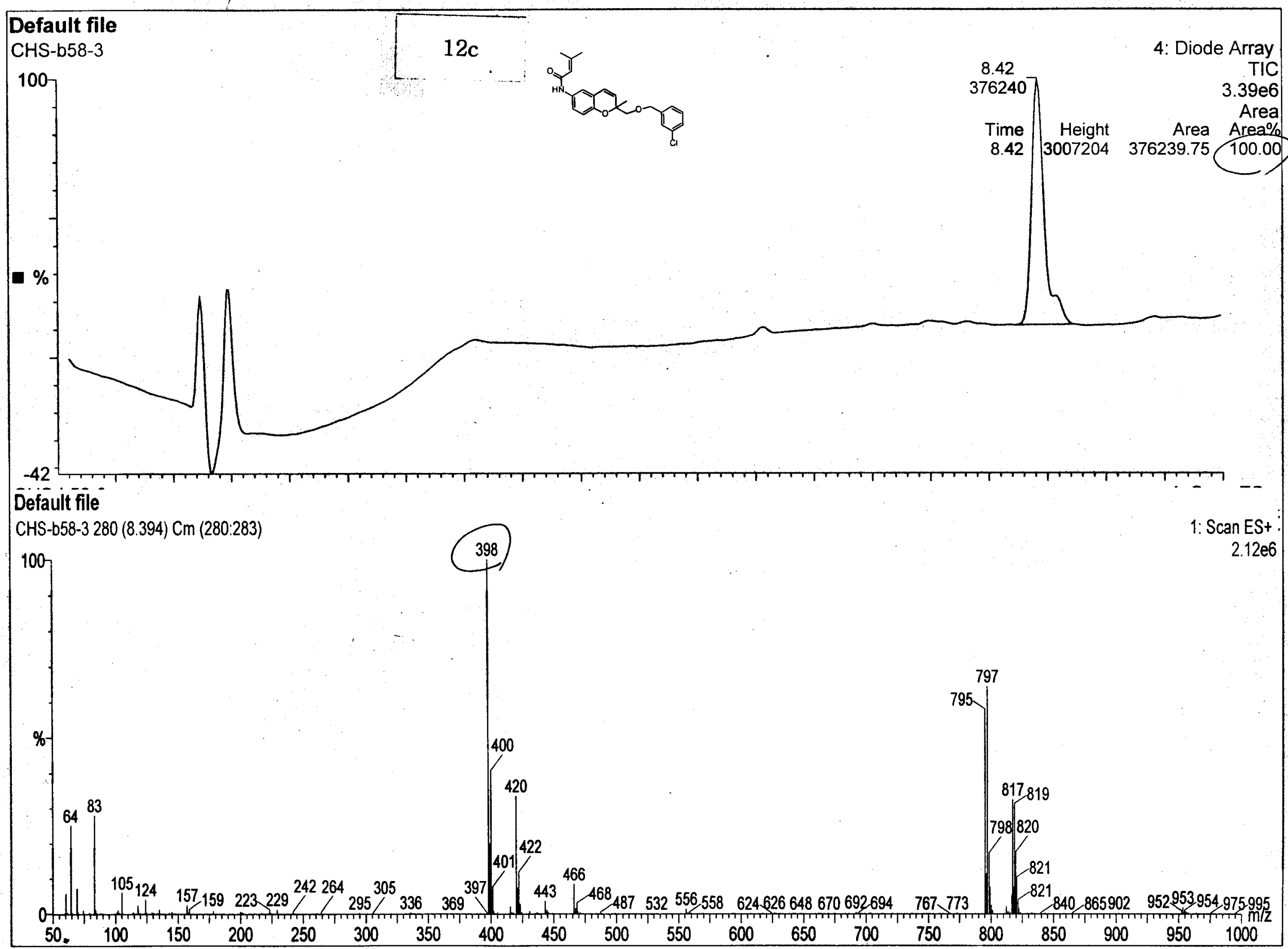




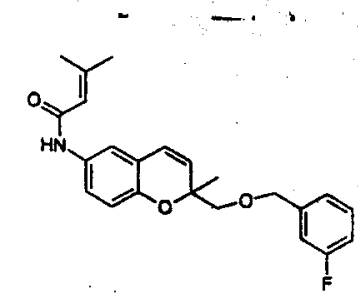

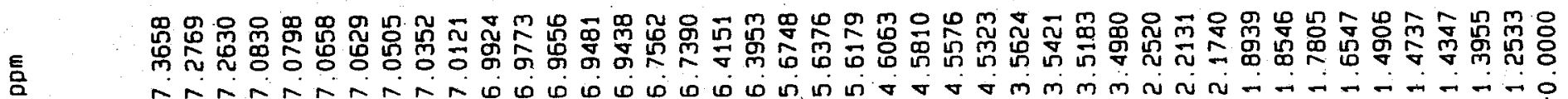

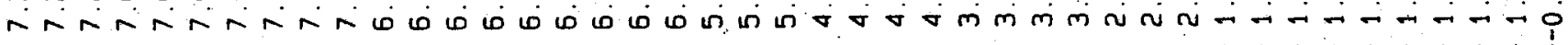
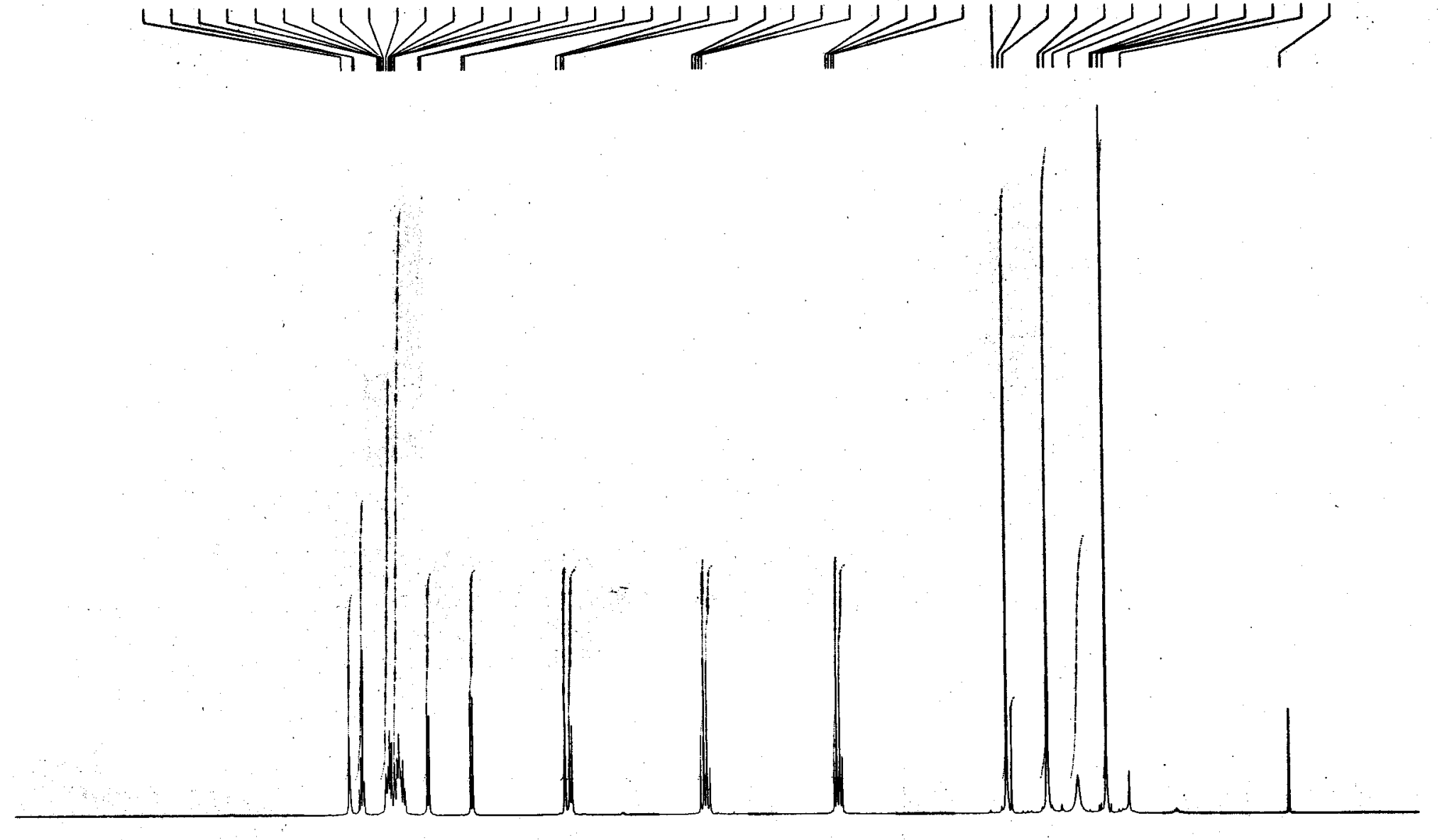

Current Data Parameters

NAME ChS-b58-5

EXPNO

PROCNO

Fz - Acquisition Parameters

Date

20040908

Time 21.50

INSTRUM spect

PROBHD $5 \mathrm{~mm}$ DUL $13 \mathrm{C}-1$

PULPROG

29
65536

SOLVENT COCI3

DS

DS

$\quad 7507.507 \mathrm{HZ}$

ODRES $\quad 0.114555 \mathrm{~Hz}$

AO $\quad 4.3648143 \mathrm{sec}$

DW

DE

TE

D1
MCAEST

128

66.600 usec

6.00 usec

$0.0 \mathrm{~K}$

$1.00000000 \mathrm{sec}$

0.00000000 sec

MCWRK $\quad 0.09500000 \mathrm{sec}$

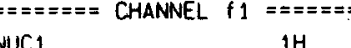
P1 10.00 use

- Processing parameters

$\begin{array}{lc}\text { SI } & 32768 \\ \text { SF } & 500.1300124 \mathrm{MHZ}\end{array}$

WOW

SSB

LB
GB

$\mathrm{GB}$
$\mathrm{PC}$

EM

0

$0.30 \mathrm{~Hz}$

10 NMR plot parameter

CX $20.00 \mathrm{~cm}$

CY $\quad 10.00 \mathrm{~cm}$

FIP $\quad 10.000 \mathrm{DOm}$

F1 $\quad 5001.30 \mathrm{~Hz}$

F2P $\quad-1.031 \mathrm{DPm}$

$0.55157 \mathrm{DO}$ $\begin{array}{lr}\text { PPMCM } & 0.55157 \mathrm{ppm} / \mathrm{cm} \\ \mathrm{HZCM} & 275.85828 \mathrm{~Hz} / \mathrm{cm}\end{array}$

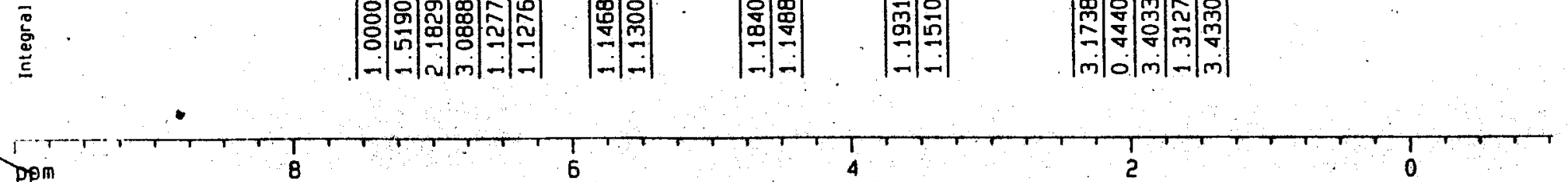




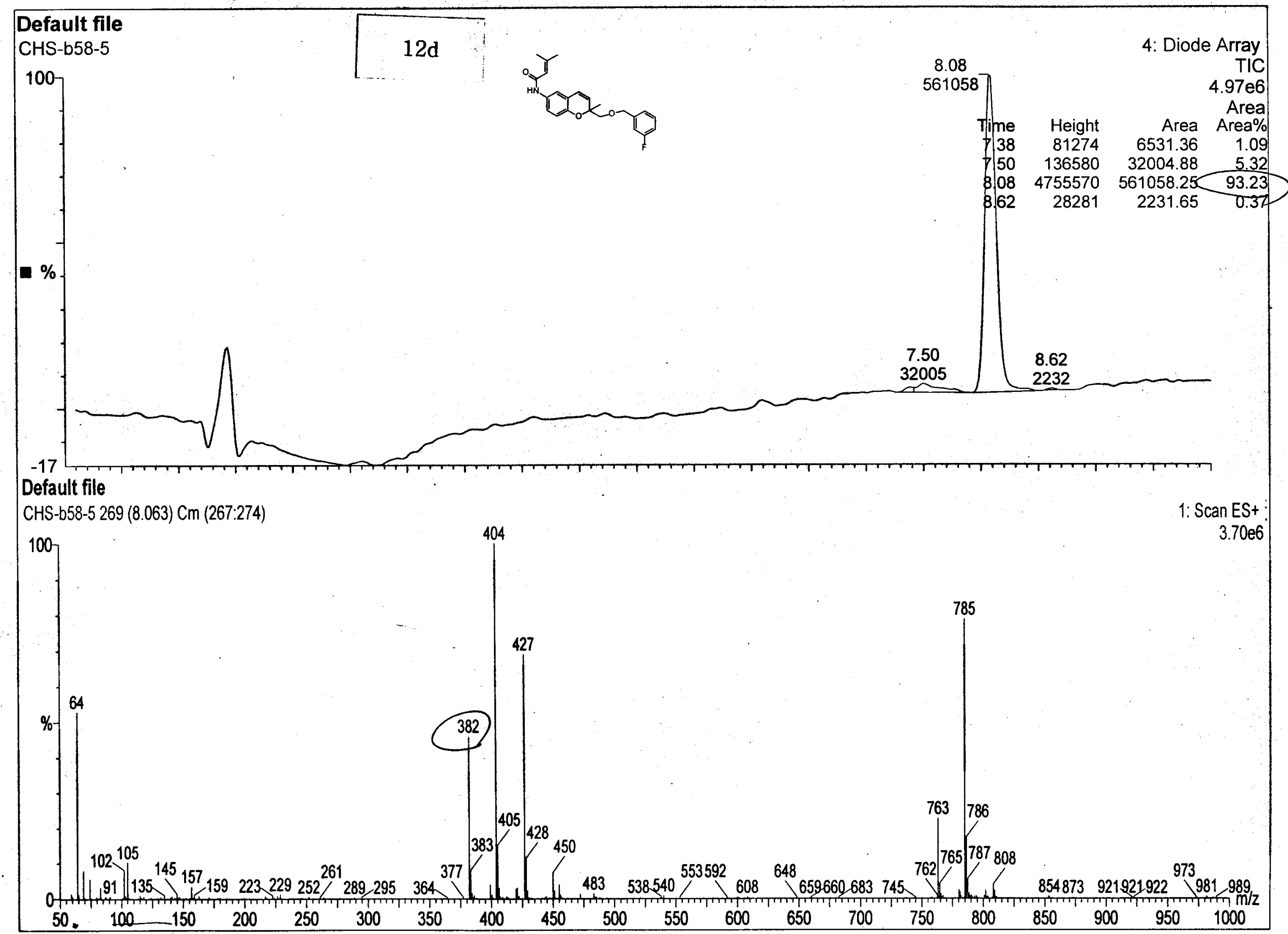




\section{$12 \mathrm{e}$}

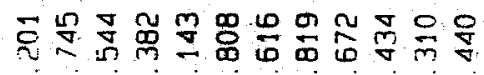

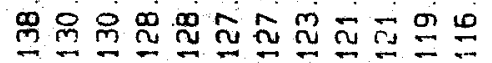

品吕额㔽曼总

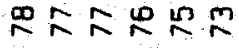
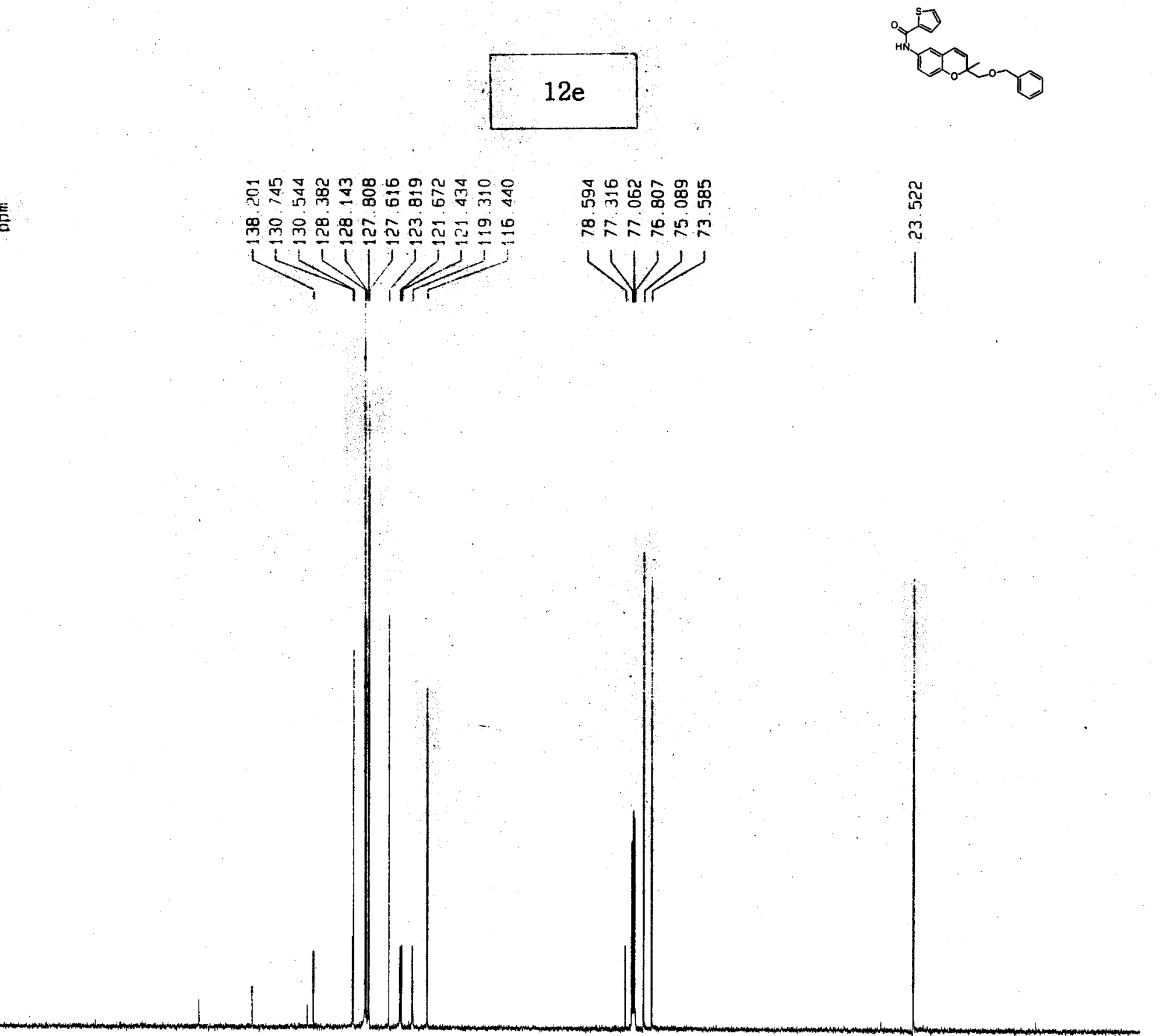

Uurrent Data Parameters

OROCNO

=2 - Acquisztion Parameter

Jate_ 20050714

Time

spect

Thinger 5 an

Pul 2906

SOLVENT $\quad 65536$

ve

JS

sith

40

40
76
36

TE

IE

in

JELIA

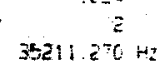

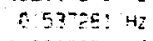

0.0306545

12800 use

000

actou see

$0.030000 \mathrm{dec}$

0

0.01500000 sec

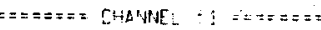

$\begin{array}{lll}2 & 130 \\ 21 & -50\end{array}$

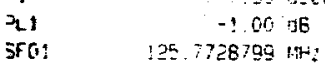

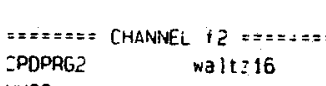

WLC2 1 IH USPC

$\mathrm{LL} 2 \quad-1.40 \mathrm{~dB}$

$\mathrm{xi3}^{\mathrm{T} 13} \quad 25.00 \mathrm{~dB}$

$5 F 0$ ? $500.1320005 \mathrm{MHz}$

$=2$ - Process ing parameters
SI 32768

$\begin{array}{lr}\text { SI } & 32768 \\ \text { SF } & 125.7577890\end{array}$

WDW

$\mathrm{B}$

$x$

10 one olot oaraneters

ix

$\begin{array}{ll}\mathrm{CX} & 20.00 \mathrm{~cm} \\ \mathrm{Cr} & 12.50 \mathrm{~cm}\end{array}$

$=1 \mathrm{p} \quad \quad 200.000 \mathrm{cmm}$
$=25151.56 \mathrm{mz}$

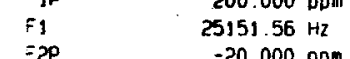

$\begin{array}{ll}-20.000 \mathrm{DPm} \\ =2 & -2515.16 \mathrm{~Hz}\end{array}$

$\begin{array}{ll}\text { SDMCM } & 11.00000 \mathrm{pDm} / \mathrm{cm} \\ \text { HZCM } & 1383.33582 \mathrm{~Hz} / \mathrm{cm}\end{array}$ 


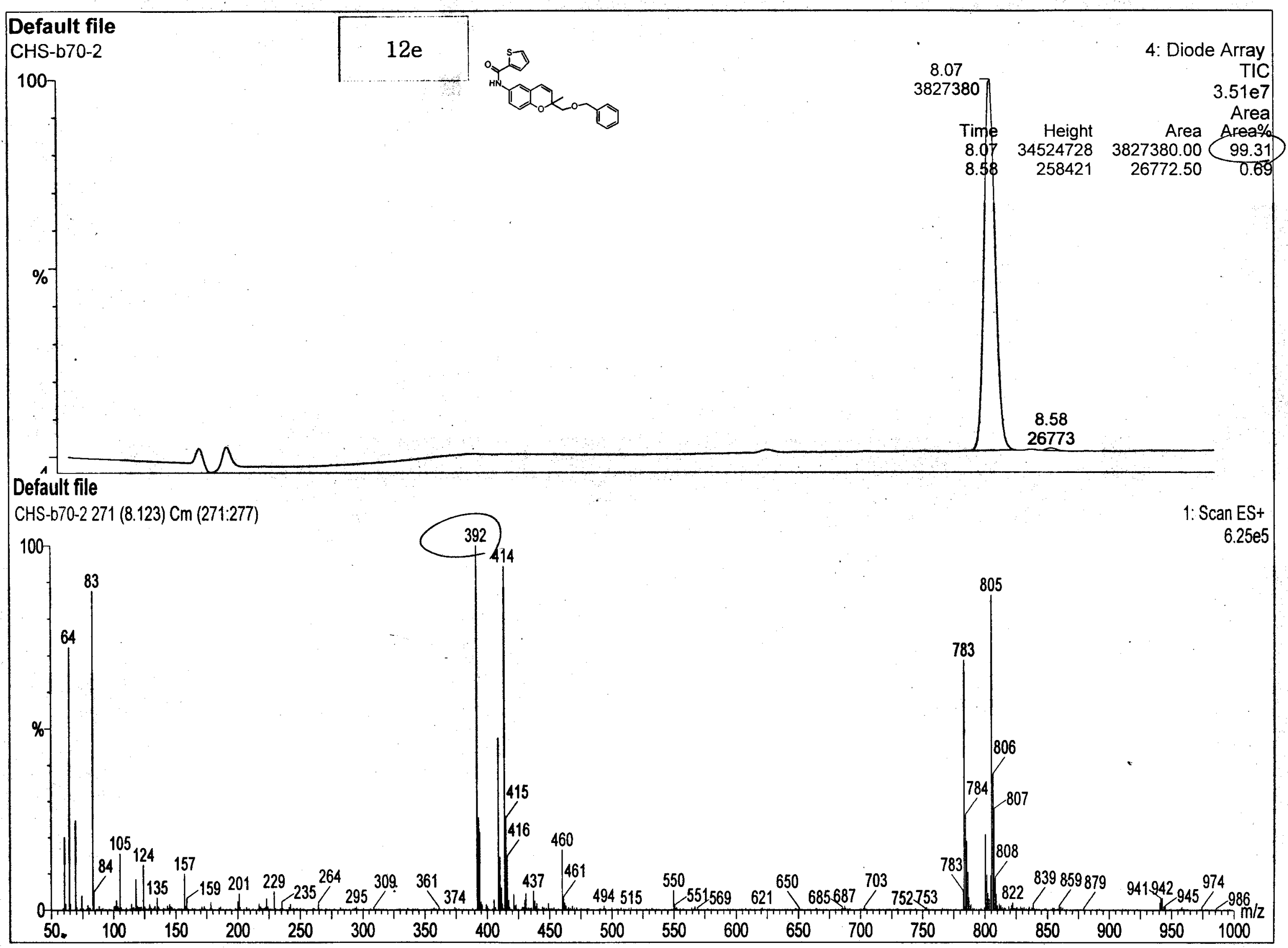



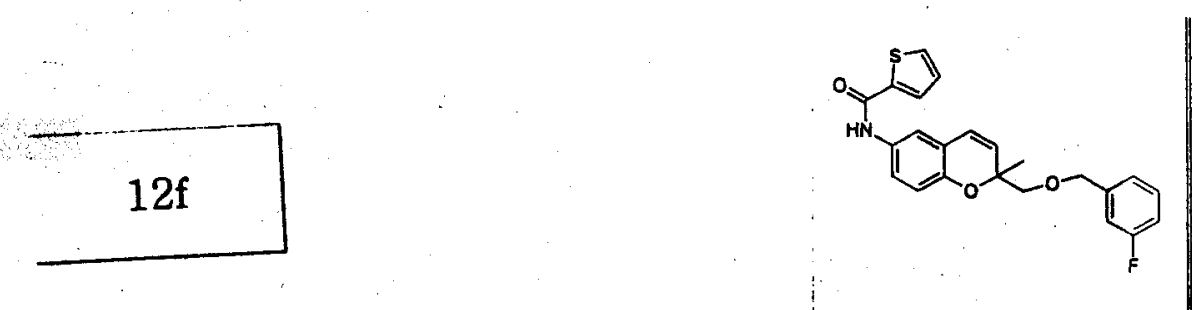

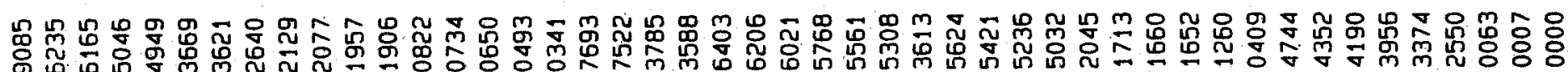

Current Data Parameters

chs $-b 70-6$

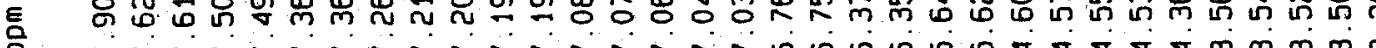

OROCNO

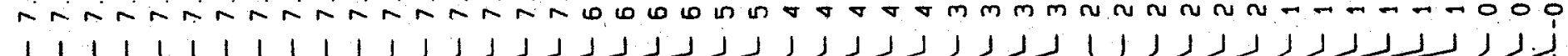

F2 - Acquisition Parameters

Date $\quad 20041013$

TIMe 17.07

INSTRUM 17.07

PROBHD $5 \mathrm{~mm}$ DUL $13 \mathrm{C}-1$

PULPROG 29

$\begin{array}{lr}\text { PULPROG } & 29 \\ \text { TD } & 65536\end{array}$

TO

SOLVENT COCl3

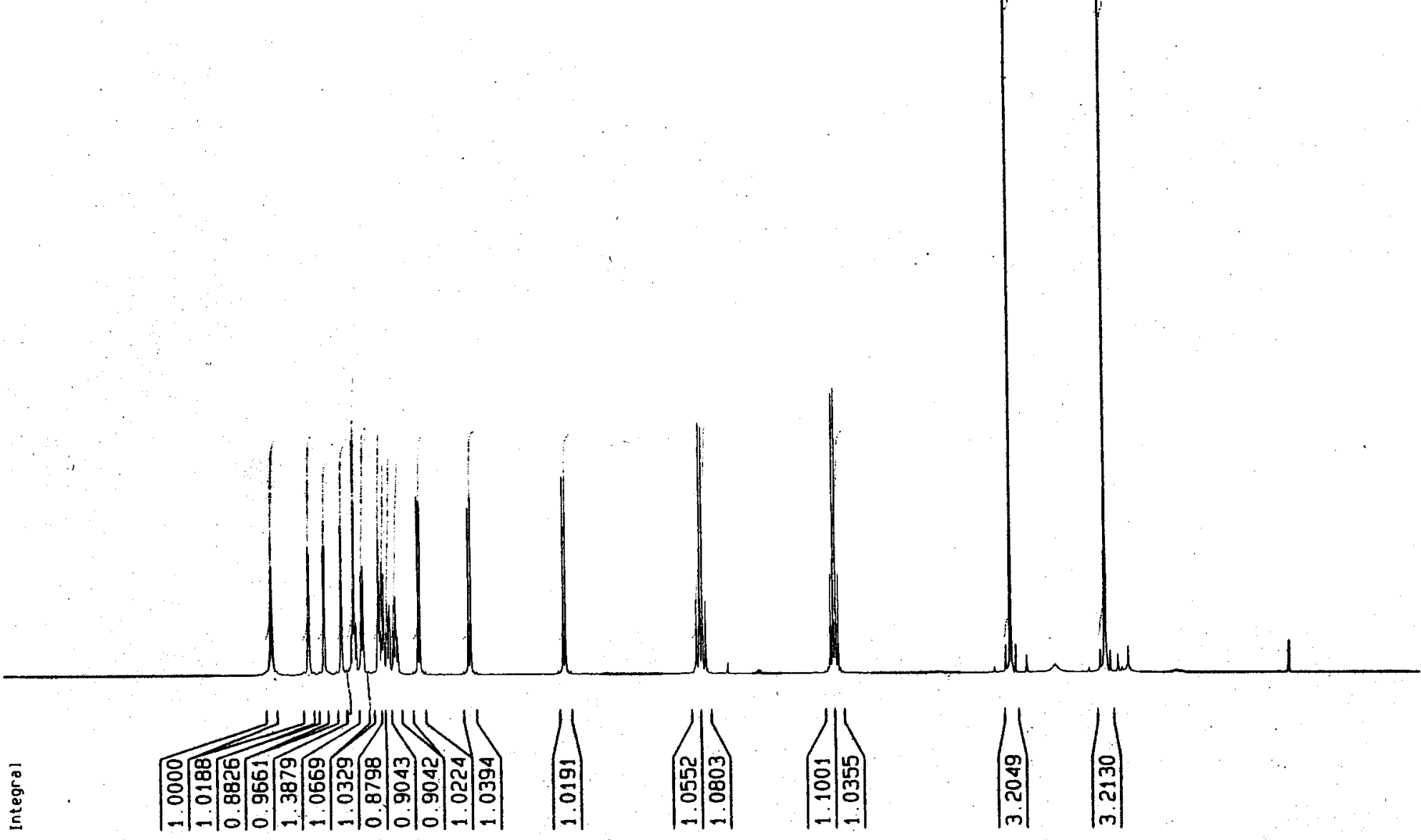

DS

SWH $\quad \begin{aligned} & 0 \\ & \text { DW }\end{aligned}$

FIDRES $\quad 0.114555 \mathrm{~Hz}$

AO $\quad 4.3648143 \mathrm{sec}$

RG

DW

DE

57

66.600 uset

6.00 use

TE

DI $1.0000000 \mathrm{sec}$

MCHest $\quad 0.01500000$ sec

$=======$ CHANNEL $f 1======$

NUC1 $1 \mathrm{H}$

$P_{1} \quad 10.00$ usec

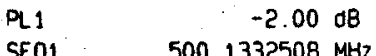

F2 - Processing parameters

51

SF $\quad 500.1300108 \mathrm{BHZ}$

WOW EM

NDW $\quad$ EM

$\begin{array}{lc}\text { LB } & 0.30 \mathrm{~Hz}\end{array}$

PC $\quad 1.00$

10 NMR DJot parameters

CX $\quad 20.00 \mathrm{~cm}$

CY $\quad 20.00 \mathrm{~cm}$

F1P $\quad 10.000 \mathrm{pom}$

F1 $5001.30 \mathrm{~Hz}$

F2P $\quad-1.030 \mathrm{DDm}$

PPMCM $\quad 0.55151 \mathrm{DDm} / \mathrm{Cm}$

$\mathrm{HZCM} \quad 275.82523 \mathrm{~Hz} / \mathrm{cm}$ 


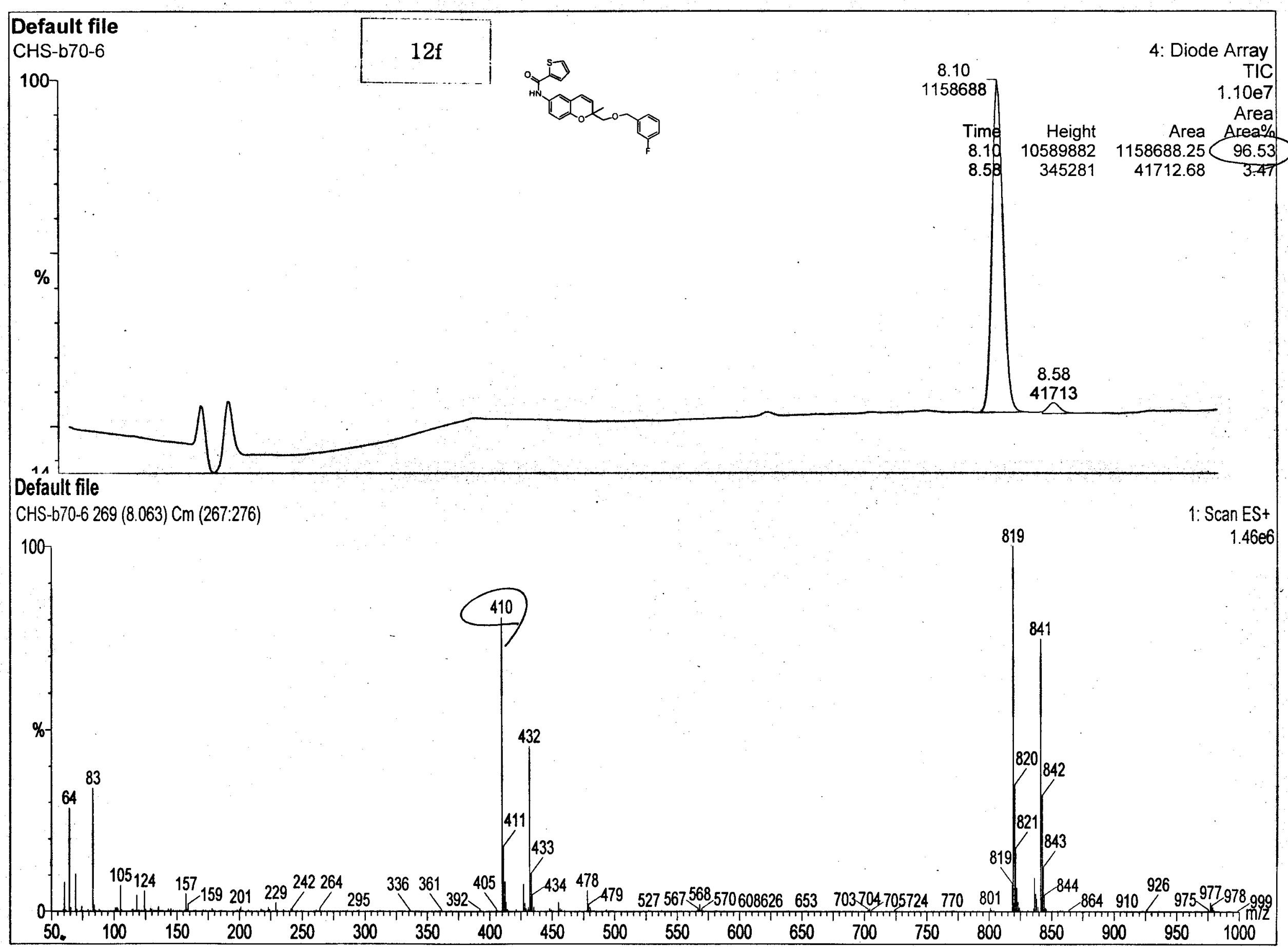




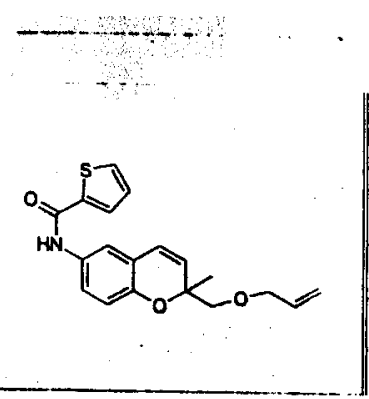

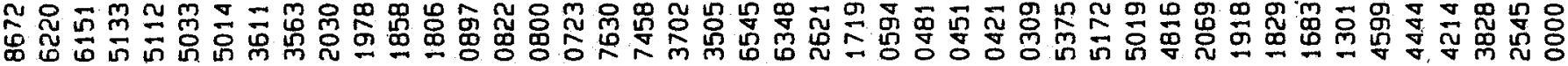
in

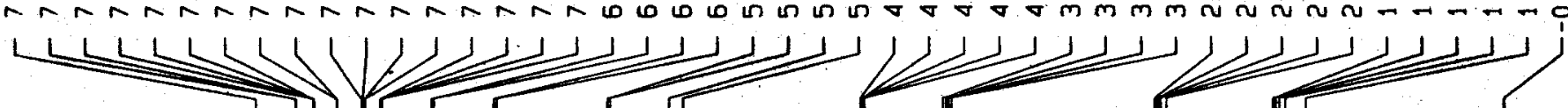

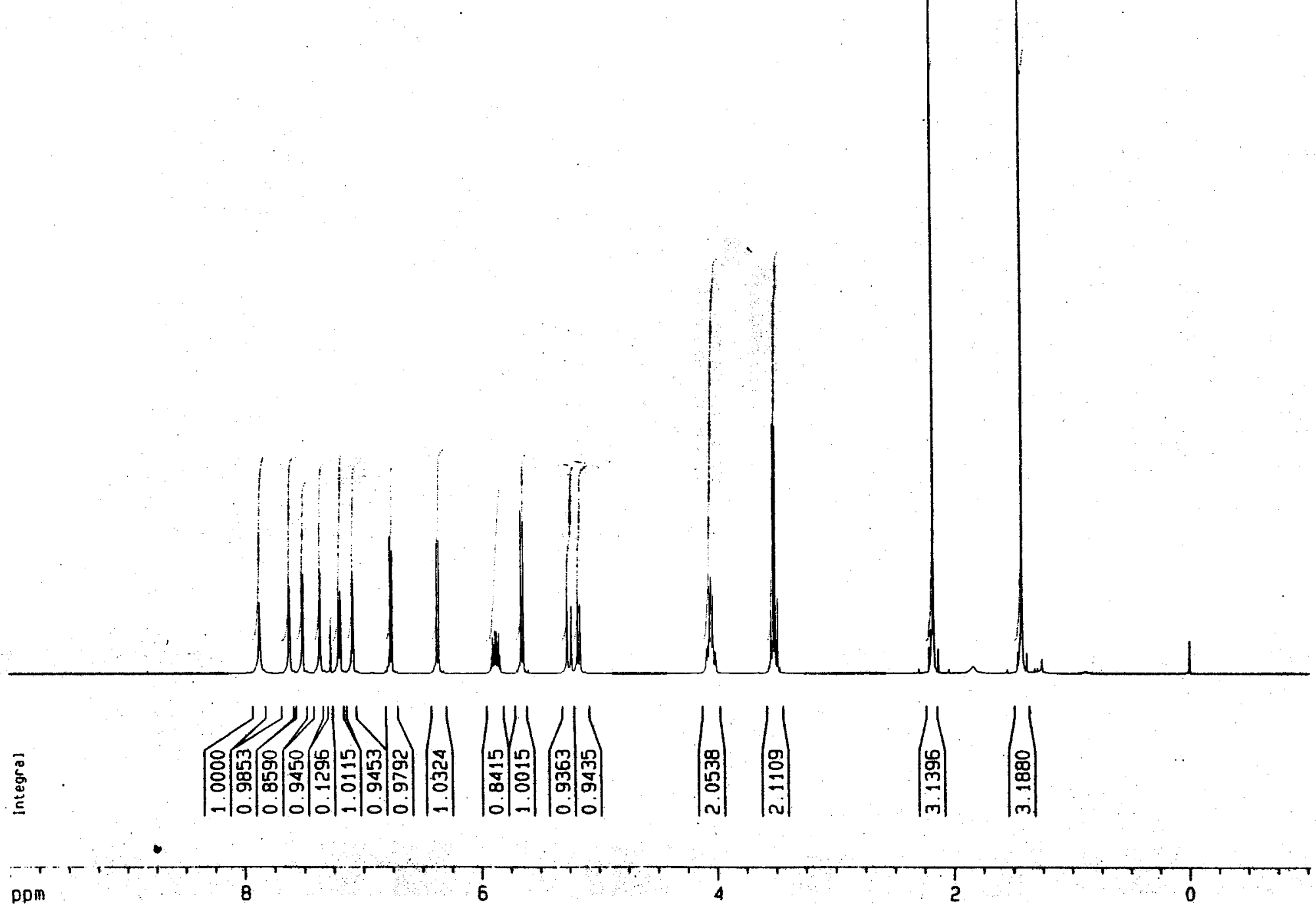

Current Data Parameters

NAME

PROCNO

chs-b70-10

F2 - Acquisition Parameters

Date.

Time

20041013

2004.31
RUM

PROBHD $5 \mathrm{~mm}$ DUL $13 \mathrm{C}-1$

PULPAOG

SOLVENT $\quad$ COC13

NS

NS

DS

FIORES

$A Q$

RG

DW

TE

DE

MCREST

0

$7507.507 \mathrm{~Hz}$

$0.114555 \mathrm{~Hz}$

4. 3648143 sec

57

66.600 usec .00 usec

00000

$.00000000 \mathrm{sec}$

0.00000000 sec

MCHAK $\quad 0.01500000$ sec

$=== \pm===-$ CHANNEL

NUC1

$P_{1} \quad 10.00$ usec

$11 \div= \pm=$
$1 H$

$5 F 01 \quad 500.133250$

F2 - Processing parameters

SI 32758

SF $\quad 500.1300085$ MHI

WOW EM

SSB

LB

GB

$E M$
0
$0.30 \mathrm{~Hz}$
0
1.00

1.00

10 NMR plot parameters

CX $20.00 \mathrm{~cm}$

Cr $\quad 20.00 \mathrm{~cm}$

FIP $\quad 10.000 \mathrm{DDm}$

$\mathrm{FI}^{2} \quad 5001.30 \mathrm{~Hz}$

$\begin{array}{lr}F 1 & 5001.30 \mathrm{~Hz} \\ F 2 P & -1.030 \mathrm{DDm}\end{array}$

F2 $\quad-515.20 \mathrm{~Hz}$

PPMCM $\quad 0.55151 \mathrm{pmm} / \mathrm{cm}$

$\mathrm{HZCM} \quad 275.82523 \mathrm{~Hz} / \mathrm{cm}$ 


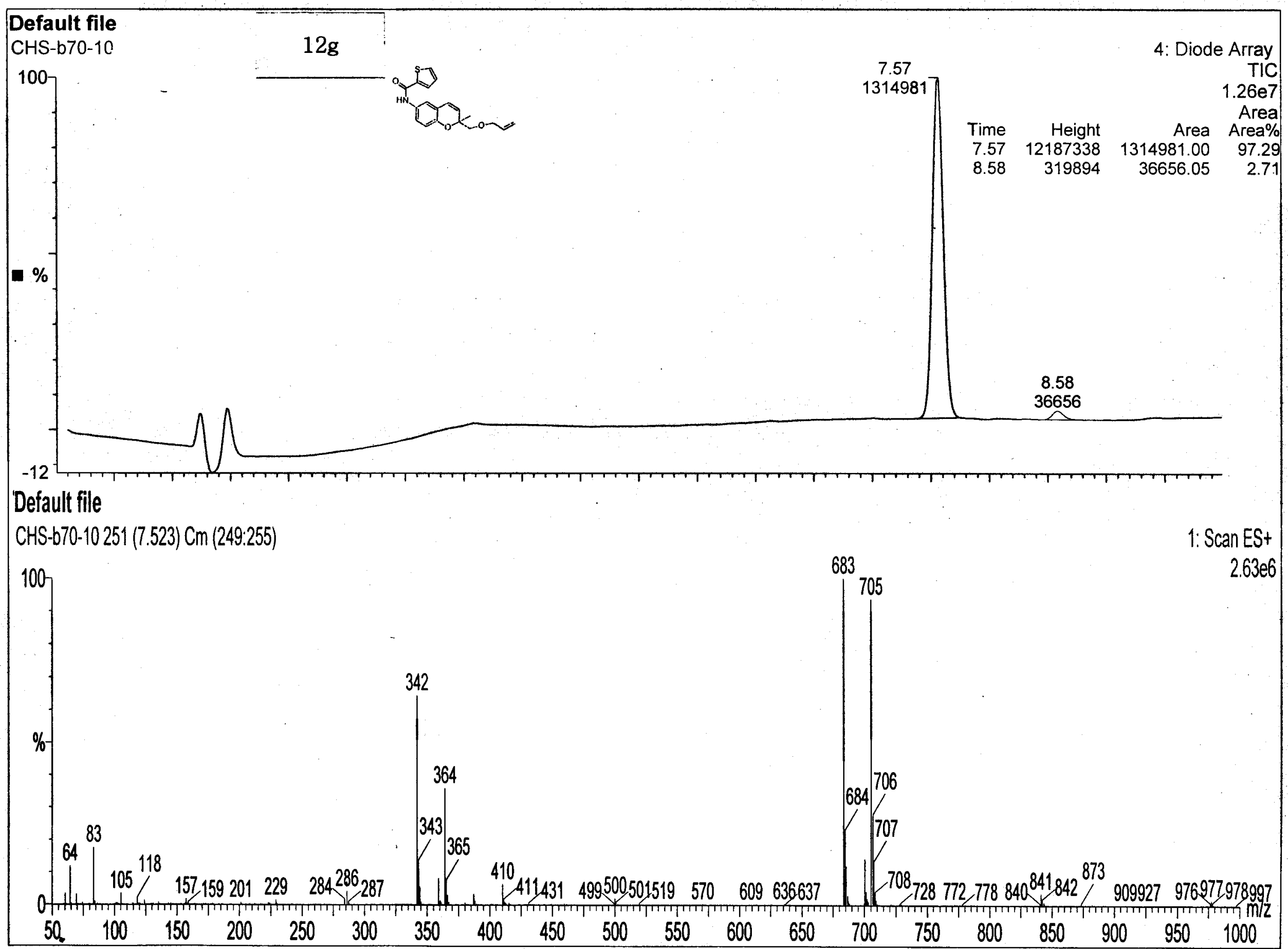




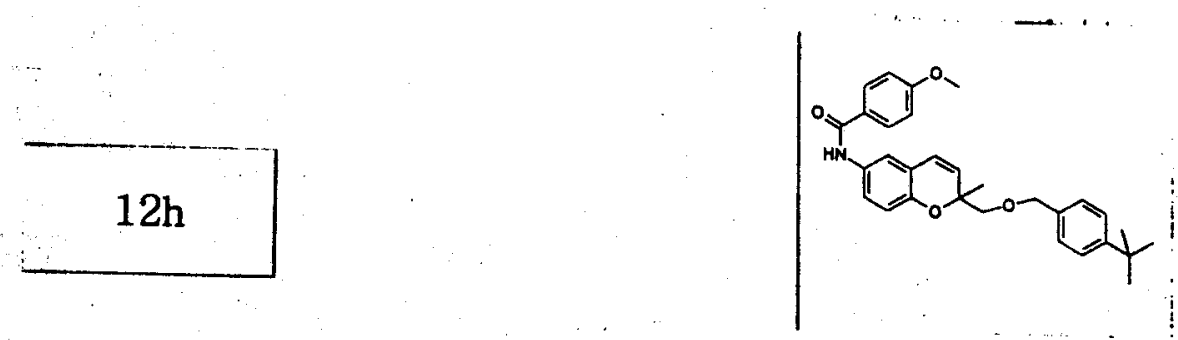

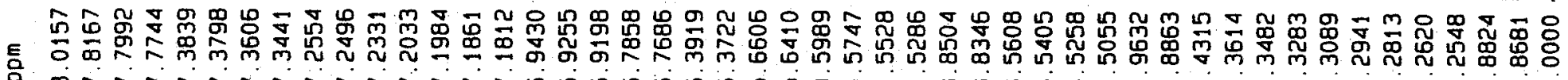

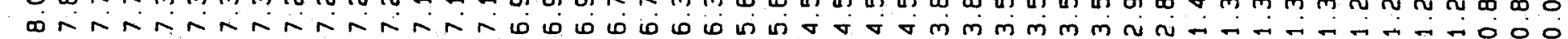

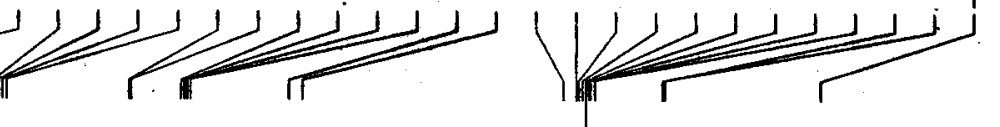

Current Data Parameters

F2 - Acquisition Parameters

Date_. 20041027

Time $\quad 0.37$

INSTRUM Spect

PROBHO $5 \mathrm{~mm}$ OUL $13 \mathrm{C}-1$

$\begin{array}{lr}\text { PULPROG } & 29 \\ \text { TD } & 65536\end{array}$

SOLVENT COCI3

DS

SWH $\quad 7507.507 \mathrm{~Hz}$

FIORES $\quad 0.114555 \mathrm{~Hz}$

AQ $\quad 4.3648143 \mathrm{sec}$

RG 57

DW 66.600 usec

DE $\quad 6.00$ use

6.00 use

D1 $\quad 1.00000000 \mathrm{sec}$

MCAEST $\quad 0.00000000 \mathrm{sec}$

MCWRK $\quad 0.01500000 \mathrm{sec}$

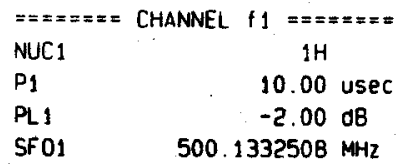

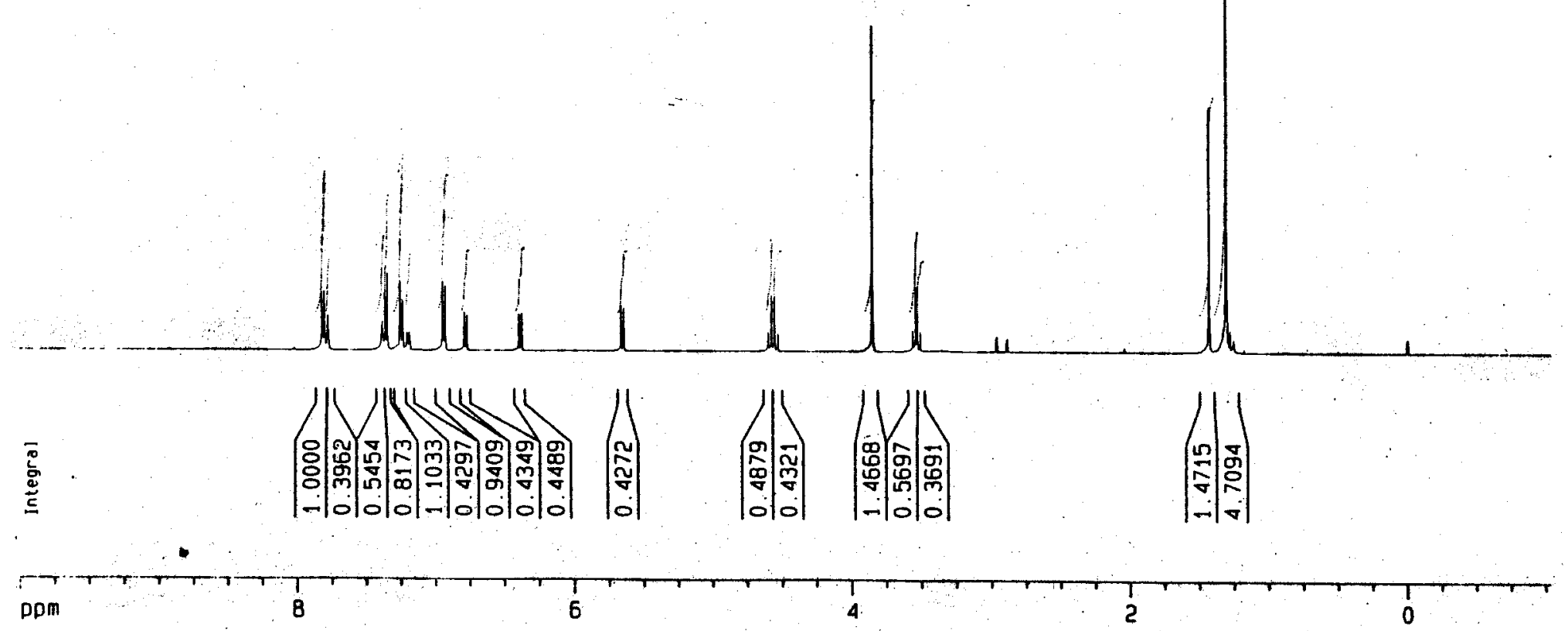

2 - Processing parameter

51 Processing paranes

SF $\quad 500.1300162 \mathrm{MHz}$

WOW EM

LB $\quad 0.30 \mathrm{HI}$

GB $\quad 0$

10 NMR plot parameters

CX $\quad 20.00 \mathrm{~cm}$

CY $\quad 12.50 \mathrm{~cm}$

$\quad 10.000 \mathrm{DDm}$

F1 $\quad 5001.30 \mathrm{~Hz}$

$\begin{array}{ll}F 2 P & -1.038 \mathrm{ppm} \\ F 2 & -519.13 \mathrm{~Hz}\end{array}$

PPMCM $\quad 0.55190 \mathrm{DDm} / \mathrm{Cm}$

HZCM $\quad 276.02127 \mathrm{~Hz} / \mathrm{cm}$ 


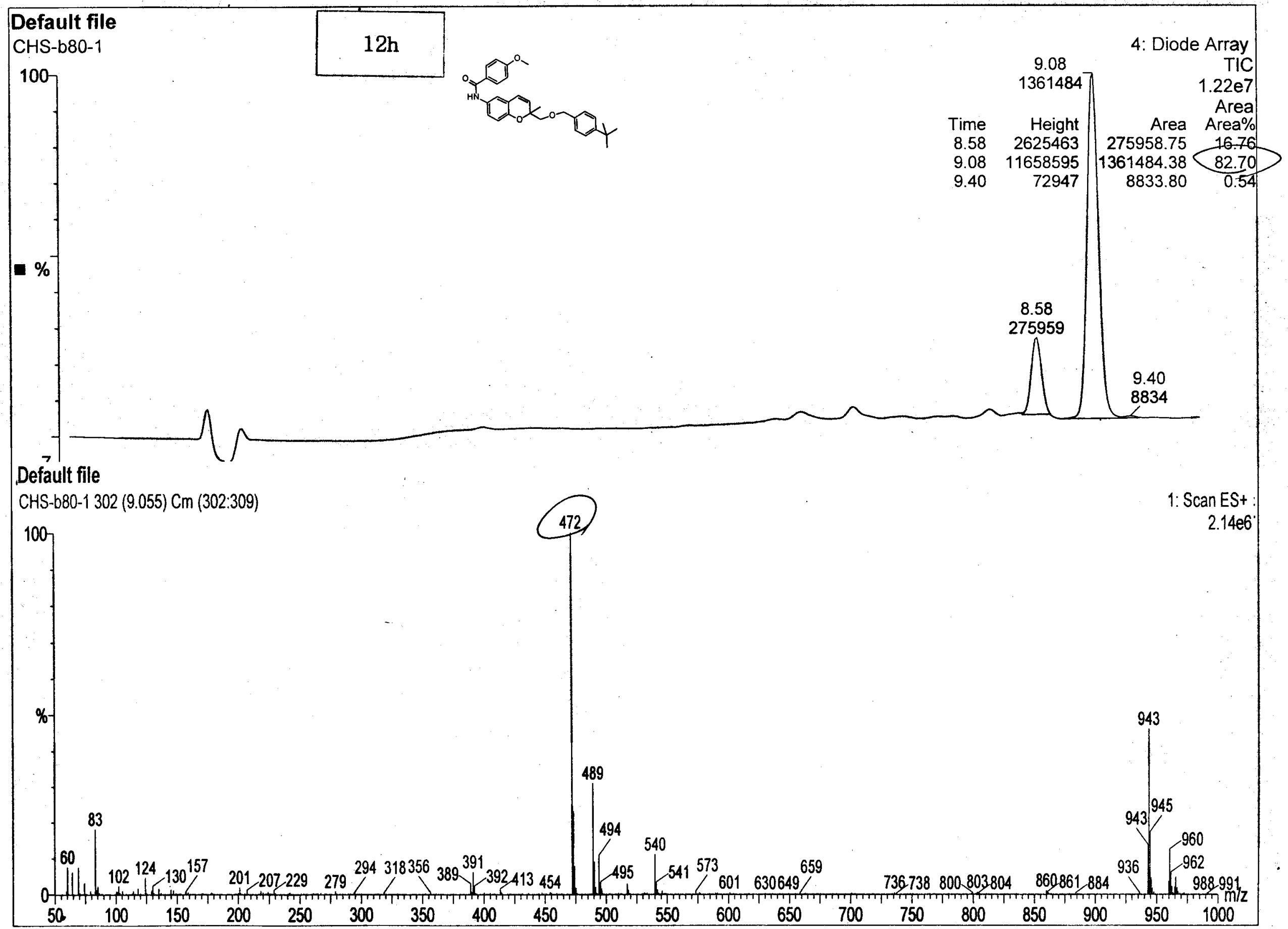




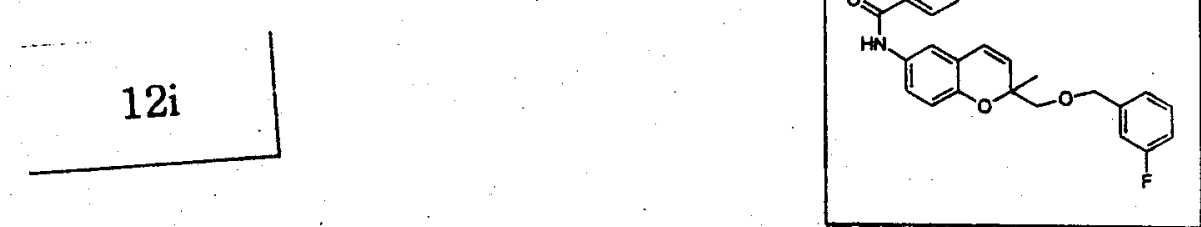

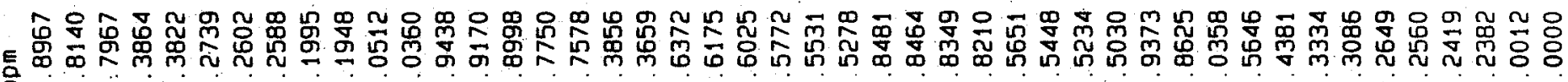

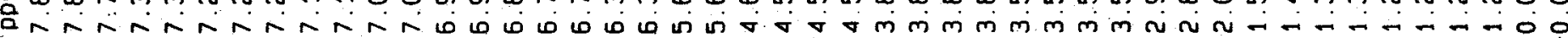
$14<5$
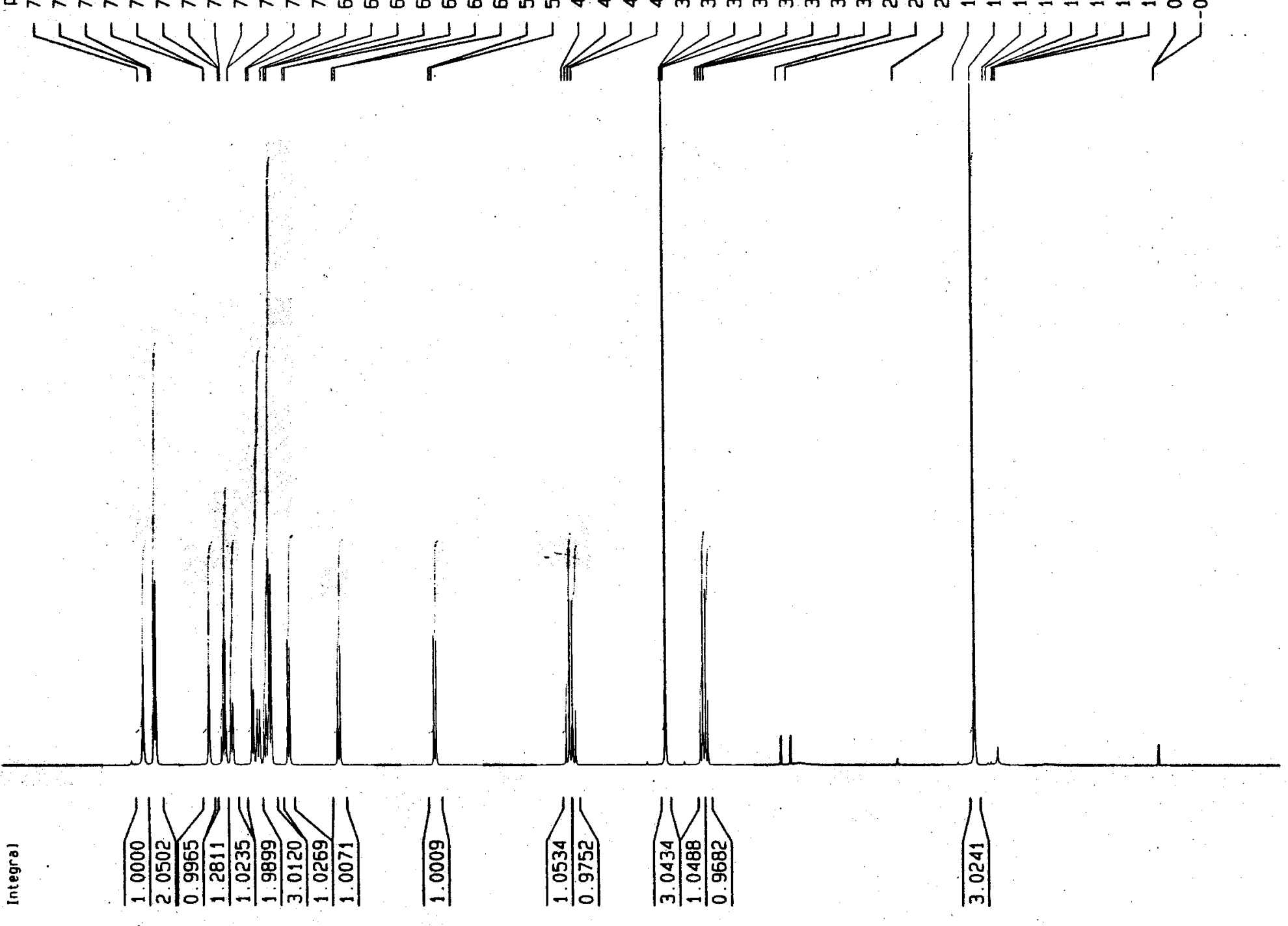

F2 - Acquisition Parameters

Date_ _ 20041026

Time

INSTRUM

19.44

PROBHD . $5 \mathrm{~mm}$ DU $13 \mathrm{C}-1$

PULPROG 2930

2930

TO 16384

SOLVENT

NS

DS
SHH
EHH $\operatorname{COCl3}$

AO $\quad \begin{aligned} & 0.413517 \mathrm{~Hz} \\ & 1.2092630 \mathrm{sec}\end{aligned}$

AG $\quad 90.5$

DW 73.800 usec

DE 6.00 usec

TI $2.00000000 \mathrm{~K}$

MCREST 0.00000000 SEC

MCREST $\quad 0.00000000 \mathrm{sec}$

$===s===$ CHANNEL $f 1==s=s==$

$\begin{array}{ll}\text { NUC1 } & 1 \mathrm{H} \\ P_{1} & 10.00 \text { use }\end{array}$

$\begin{array}{ll}P 1 & 10.00 \text { use } \\ \text { PL1 } & -2.00 \mathrm{~dB}\end{array}$

SFO1 $500.1329272 \mathrm{MHz}$

F2 - Processing parameters

SI 32768

SF $\quad 500.1300141 \mathrm{MHZ}$

WOW EM

$\begin{array}{lr}\text { SSB } & 0 \\ \text { LB } & 0.30 \mathrm{HZ}\end{array}$

$\mathrm{GB} \quad 0$

10 NMR plot parameters

CX $\quad 20.00 \mathrm{~cm}$

CY $\quad 12.50 \mathrm{~cm}$

$\begin{array}{lr}\text { F1P } & 9.000 \mathrm{DDm} \\ \mathrm{F}_{1} & 4501.17 \mathrm{~Hz}\end{array}$

F2P - $-1.031 \mathrm{pDm}$

F2

$\begin{array}{lr}\text { PPMCM } & 0.50154 \mathrm{Dpm} / \mathrm{cm} \\ \mathrm{HZCM} & 250.83675 \mathrm{~Hz} / \mathrm{cm}\end{array}$

$-515.56 \mathrm{~Hz}$ 


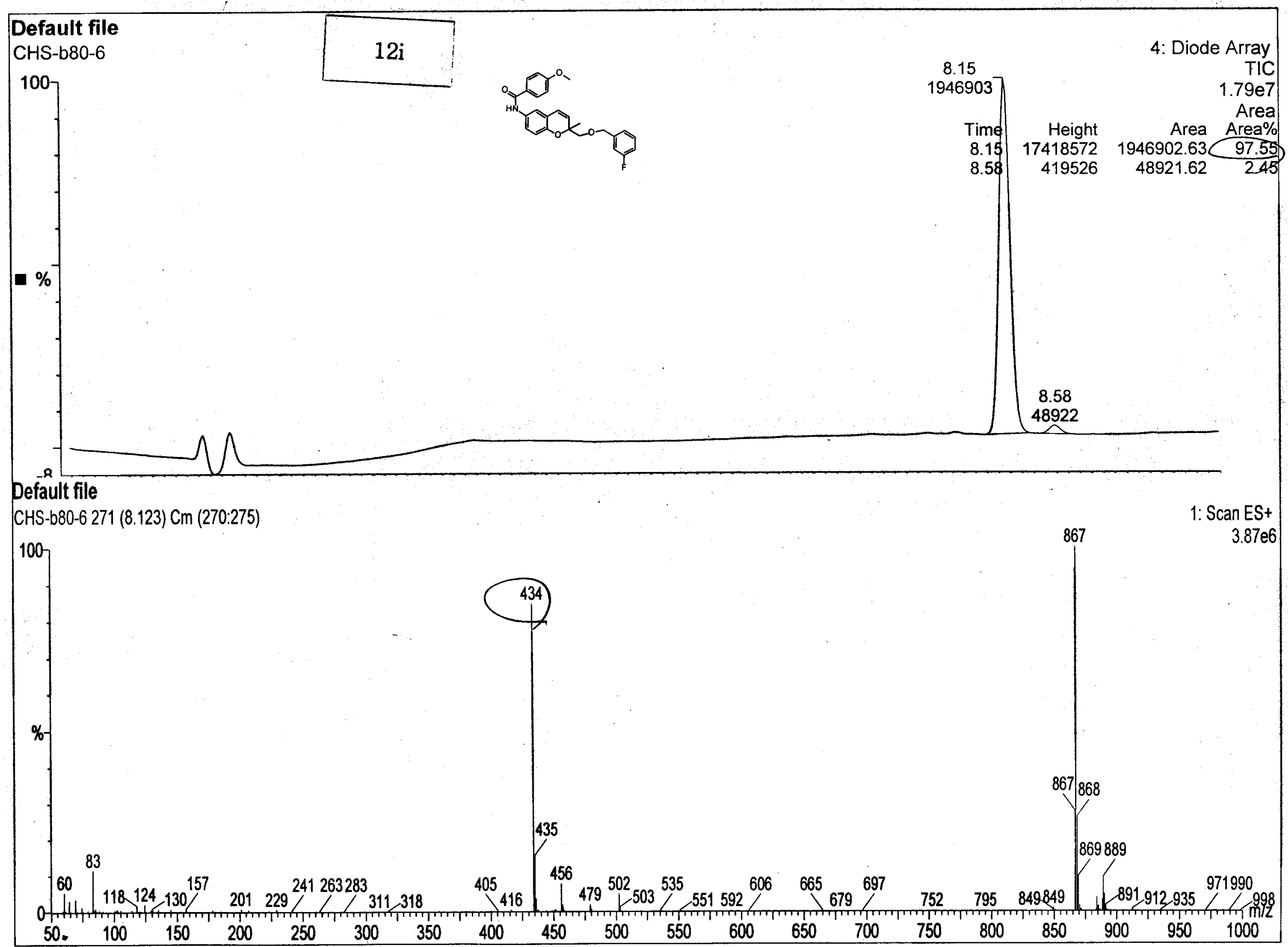




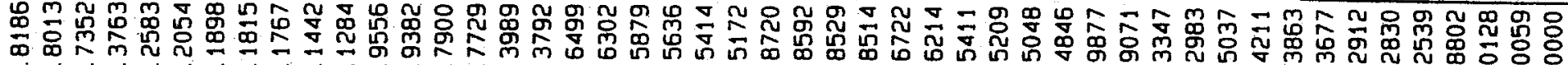

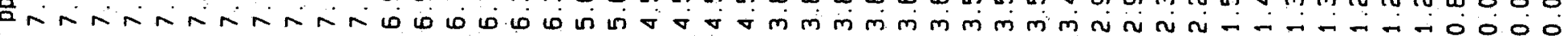
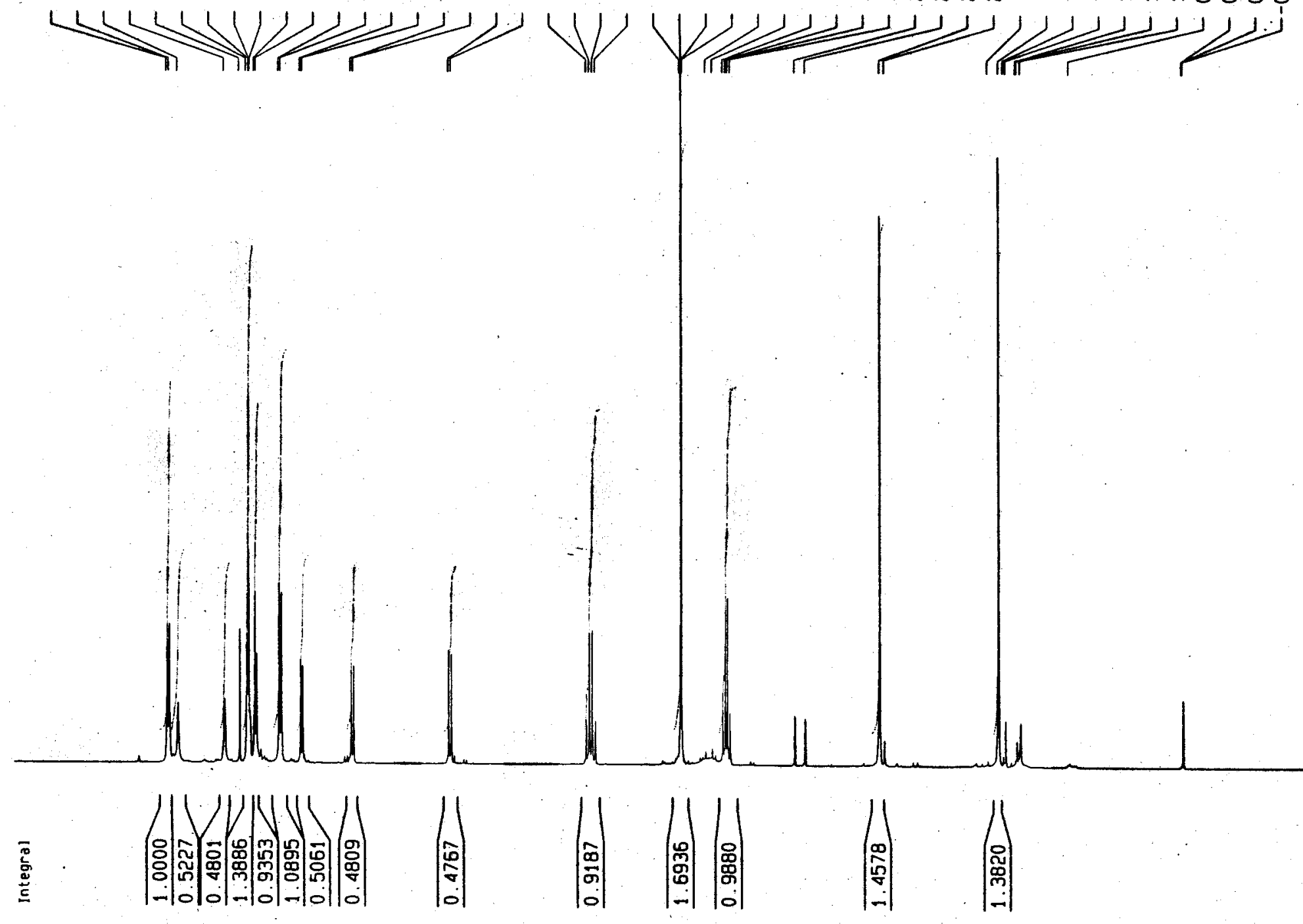


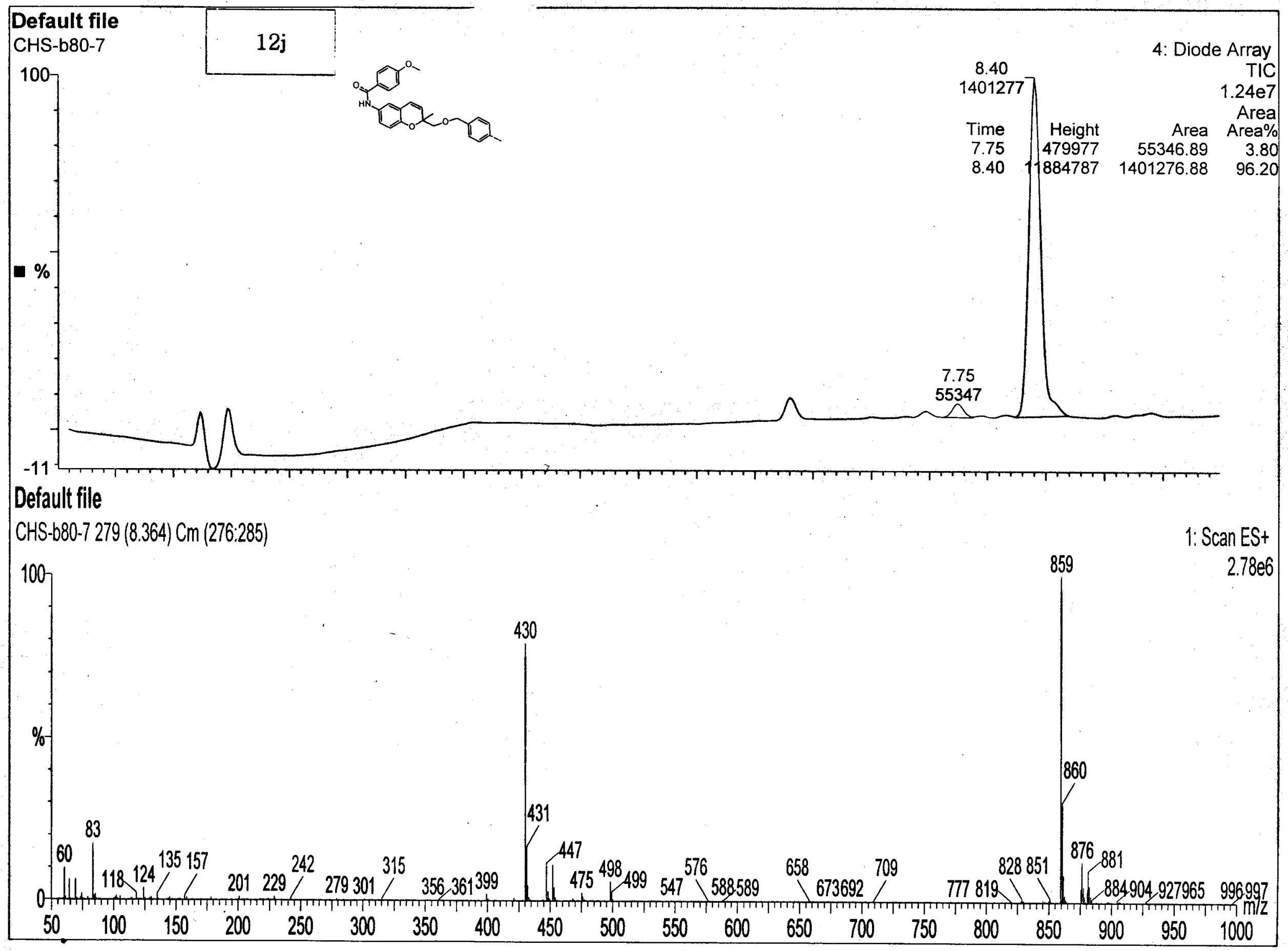




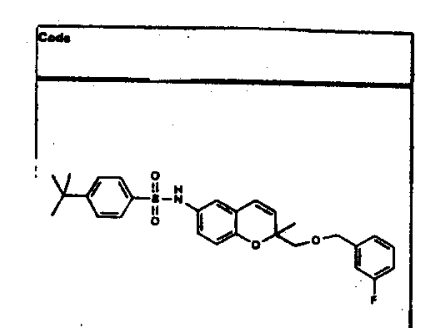

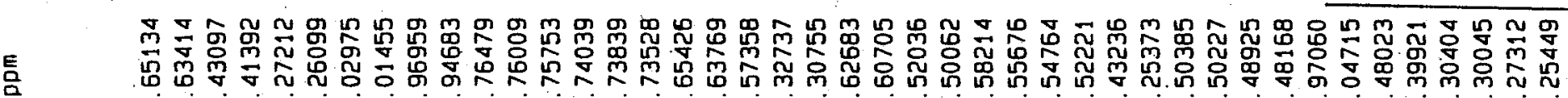

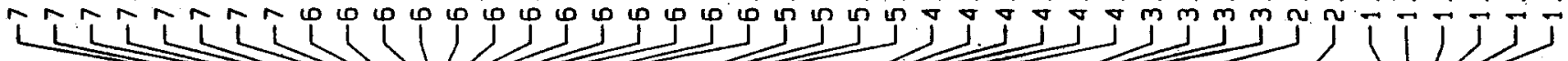
71 in ता

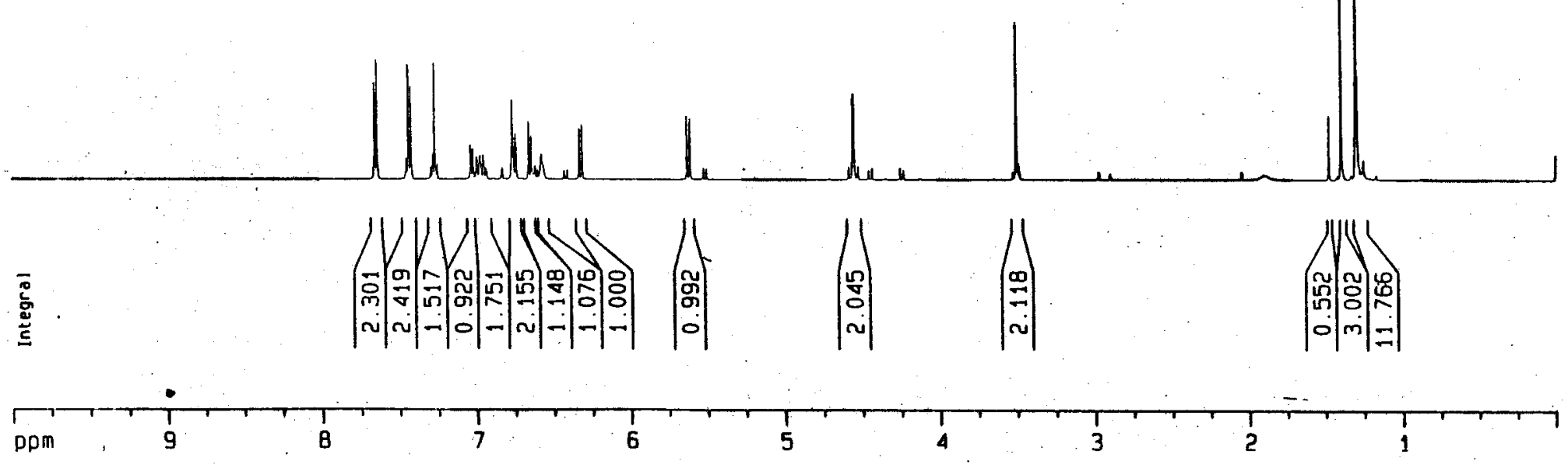

Current Data Parameters chs-b102a-G PROCNO

F2 - Acquisition Parameters Date_ 20041116

$\begin{array}{rr}\text { Time } & 200411.51\end{array}$

INSTRUM 5 Spect PROBMD 5 mIm DUL 13C-1 PULPROG 5 $\begin{array}{lr}\text { PULPROG } & 29 \\ \text { TO } & 65536\end{array}$ $\begin{array}{ll}\text { TO } & \text { GLLVNT } \\ \text { COC13 }\end{array}$ NS $\begin{array}{lc}\text { DS } & 0 \\ \text { SHH } & 7507.507 \mathrm{~Hz}\end{array}$ FIORES $\quad 0.114555 \mathrm{~Hz}$ AQ 4. 3648143 sec AG 54 DW 66.600 usec DE $\quad 6.00$ usec TE MCRESir $\quad 0.00000000 \mathrm{sec}$ MCREST 0.000000 sec $0.01500000 \mathrm{se}$

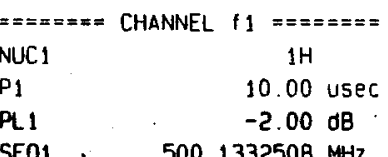

Thocessing oaraneters

F2 - Processing Darameters $\begin{array}{lc}\text { SI } & 32768 \\ \text { SF } & 500.1300135 \mathrm{MHZ}\end{array}$ WOW $\quad 500.1300135$ $\begin{array}{lc}\text { SSB } & 0 \\ \text { LB } & 0.30 \mathrm{~Hz}\end{array}$ $\begin{array}{lr}68 & 0 \\ P C & 1.00\end{array}$

10 NMR plot parameters

$\begin{array}{lc}\text { CX } & 20.00 \mathrm{~cm} \\ \text { CY } & 17.00 \mathrm{~cm} \\ \text { F1P } & 10.000 \mathrm{pDm} \\ \text { F1 } & 5001.30 \mathrm{~Hz} \\ \text { F2P } & 0.000 \mathrm{pDm} \\ \text { F2 } & 0.00 \mathrm{~Hz} \\ \text { PPMCM } & 0.50000 \mathrm{ppm} / \mathrm{cm} \\ \text { HZCM } & 250.06500 \mathrm{~Hz} / \mathrm{cm}\end{array}$




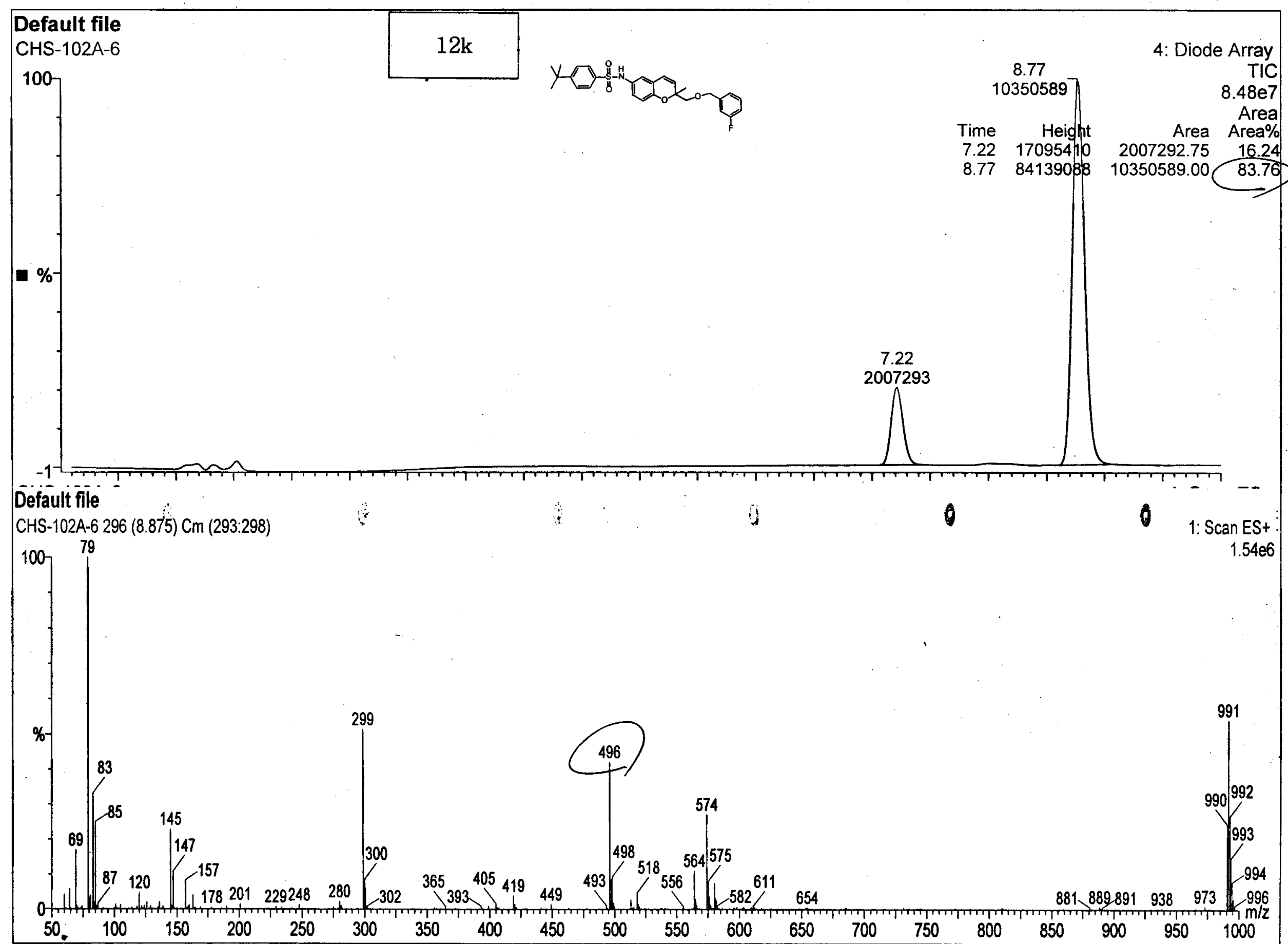




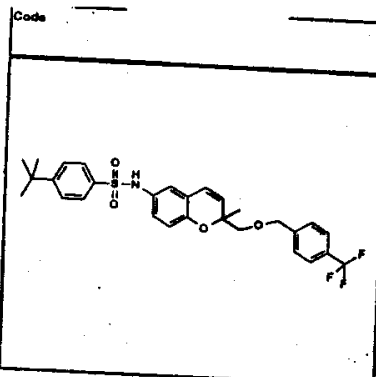

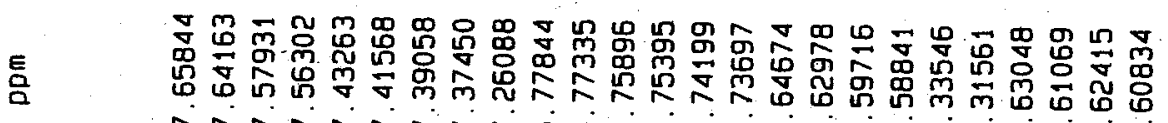

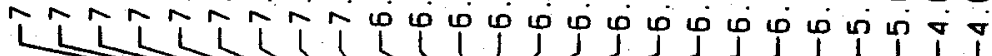
ता

品品 的圆

产宁哭器

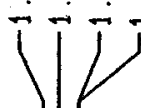

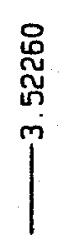
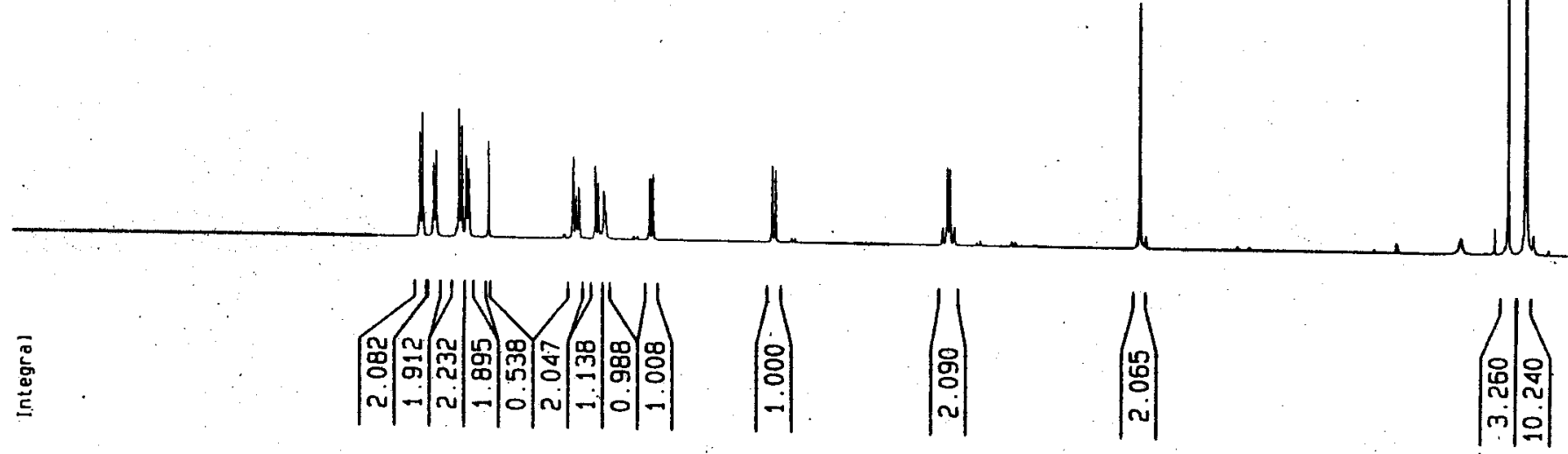

Current Data Parameters NAME

EXPNO

PROCNO

F2 - Acquisition Parameters

Date_ 20041116

Time 22.00

INSTRUM spect.

PROBHD $5 \mathrm{~mm}$ DUL $13 \mathrm{C}-1$

PULPROG 29

SOL VENT

NS

DS

FIDRES

$A Q$

RG

DW

DE

D1

MCRES

MCWAK

$=$

NUC1

P1

$\begin{array}{ll}P L 1 & 10.00 \text { usec } \\ & -2.00 \mathrm{~dB}\end{array}$

SF01 $500.1332508 \mathrm{MHZ}$

F2 - Processing parameters

SI Process ing paraneters

SF $\quad 500.1300135 \mathrm{MHz}$

WOW

SSB

LB

$\mathrm{GB}$
$\mathrm{PC}$

EM

$E M$
0
$0.30 \mathrm{~Hz}$
0

0
1.00

10 NMR plot parameters

CX $\quad 20.00 \mathrm{~cm}$

CY $\quad 17.00 \mathrm{~cm}$

F1P $\quad \begin{array}{ll}10.000 \mathrm{ppm} \\ \mathrm{Fi} & 5001.30 \mathrm{~Hz}\end{array}$

F2P $\quad \begin{array}{ll}5001.30 \mathrm{~Hz} \\ \text { F2 }\end{array}$

F2

PPMCH

$\mathrm{HZCM}$

$0.00 \mathrm{~Hz}$
$50000 \mathrm{pom} / \mathrm{cm}$

$250.06500 \mathrm{~Hz} / \mathrm{cm}$

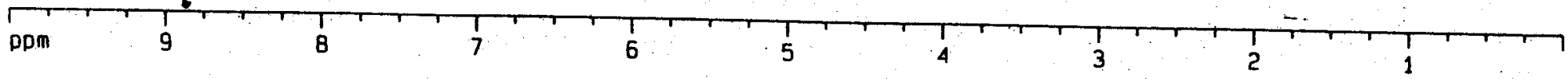




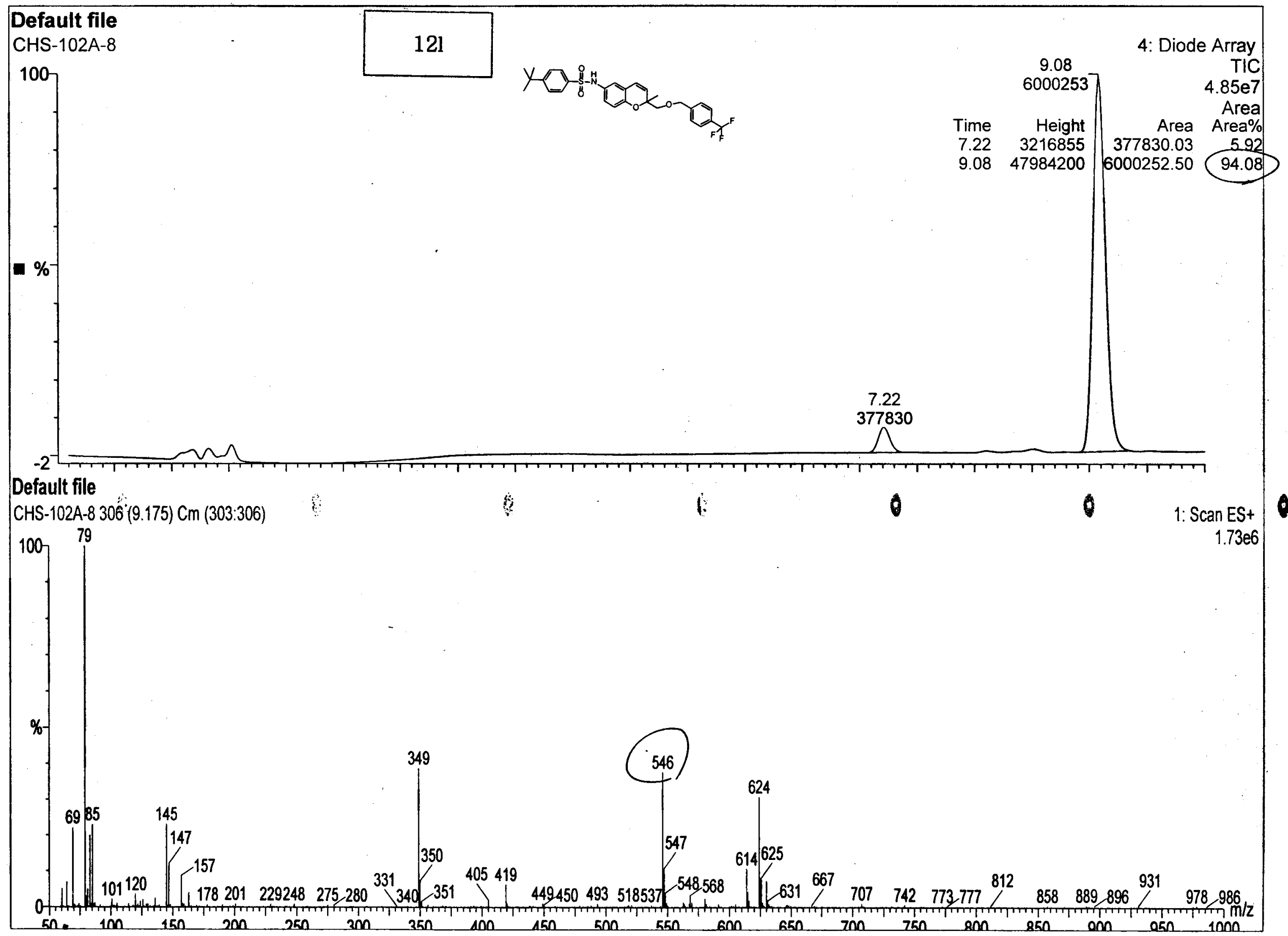




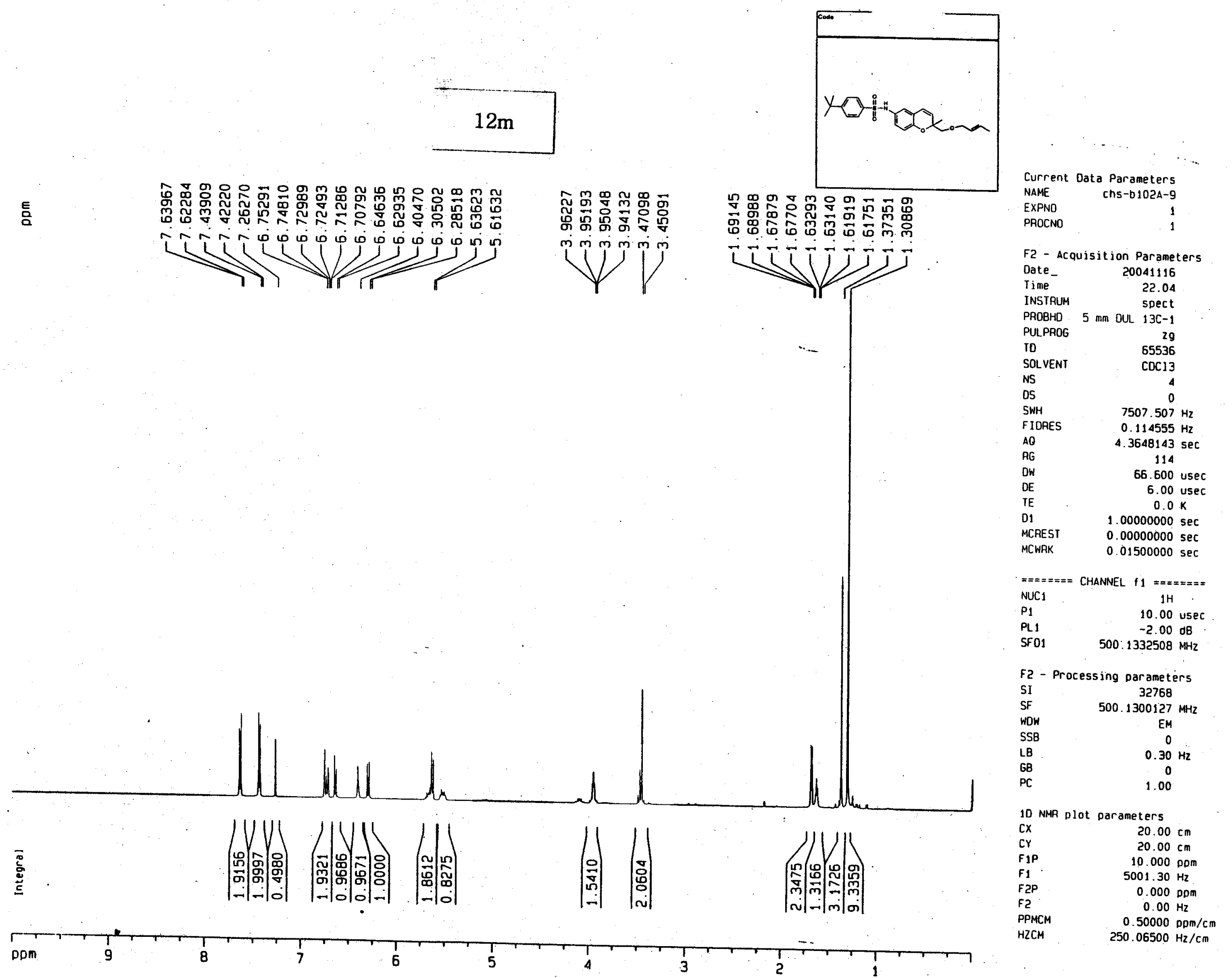



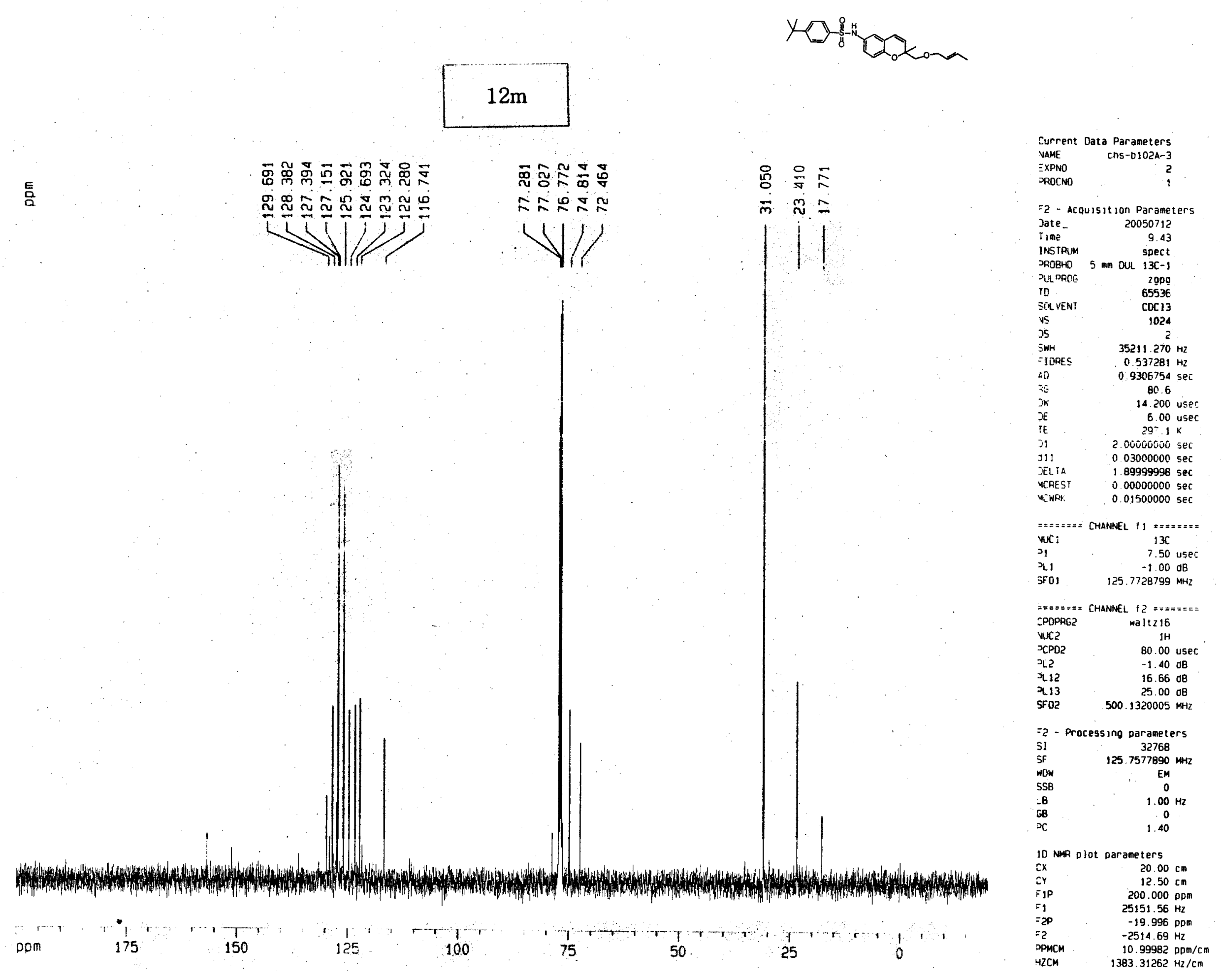


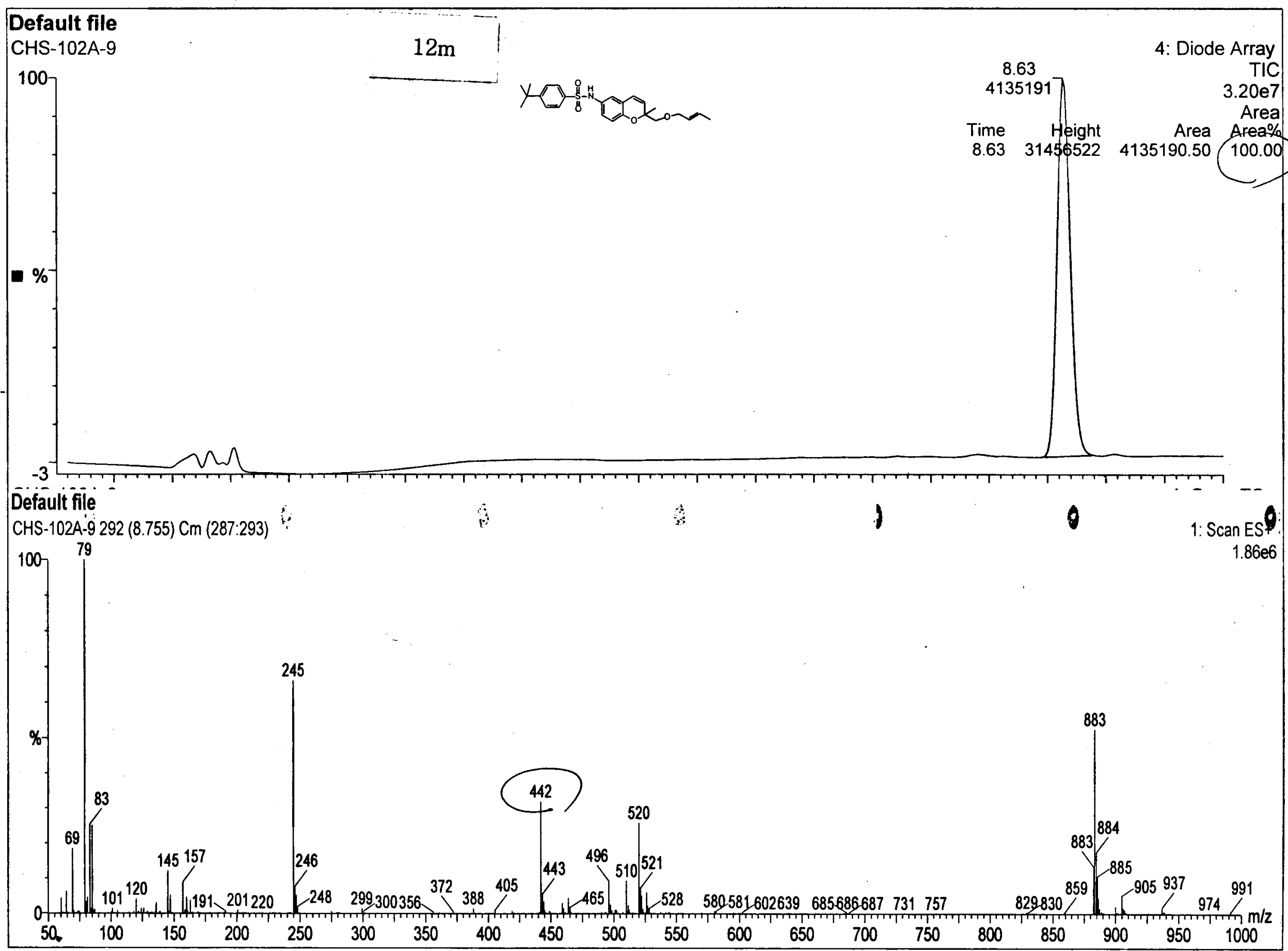




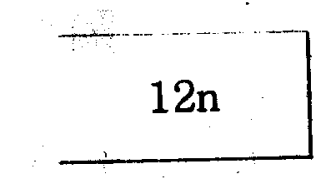

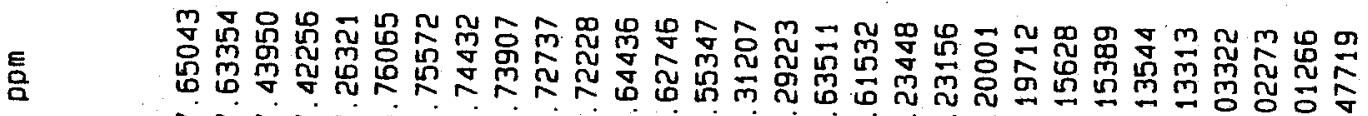
ᄂ

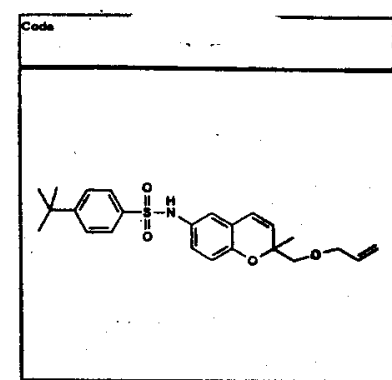

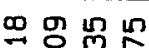

要离总品

रा.

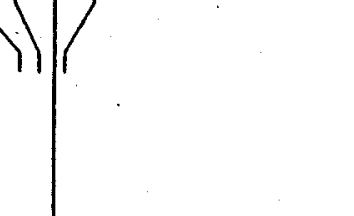

Current Data Parameters NAME chs-b102A-10 PAOCNO

F2 - Acquisition Parameters

Date

20041116

22.09 PROBHO $5 \mathrm{~mm}$ DUL $13 \mathrm{C}-1$ 5 min DUL $130-1$

SOL VENT

NS

DS

SWH

FIO

AO

AG

DW

TE

MCRES

MCREST
MCWRK

$\begin{array}{r}29 \\ 65536 \\ \hline\end{array}$

$\operatorname{CDC} 33$ 0

$7507.507 \mathrm{~Hz}$

$0.114555 \mathrm{~Hz}$

4. 3648143 sec 114.

66.600 usec 6.00 usec

1.0000000

$1.00000000 \mathrm{sec}$

$0.00000000 \mathrm{sec}$

$z=z=z==$ CHANNEL $f 1$ $=z====$

NUC:

P1 10.00 usec

SF01 $\quad 500.000$

F2 - Processing parameters

SI 32768

SF $\quad 500.1300123 \mathrm{MHz}$

WDW

SSB

LB

$\mathrm{PC}$

EM

0
$0.30 \mathrm{~Hz}$
0

100

10 NMR plot parameters

CX

CY $\quad 20.00 \mathrm{~cm}$

F1P $\quad 10.000 \mathrm{pDm}$

F1 $\quad 5001.30 \mathrm{~Hz}$

F 1 P

F2

PPMCM

$0.000 \mathrm{ppm}$

$0.00 \mathrm{~Hz}$

$\mathrm{HZCM} \quad 0.50000 \mathrm{ppm} / \mathrm{cm}$

$250.06500 \mathrm{~Hz} / \mathrm{cm}$

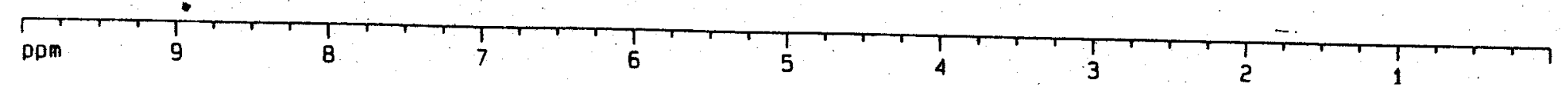




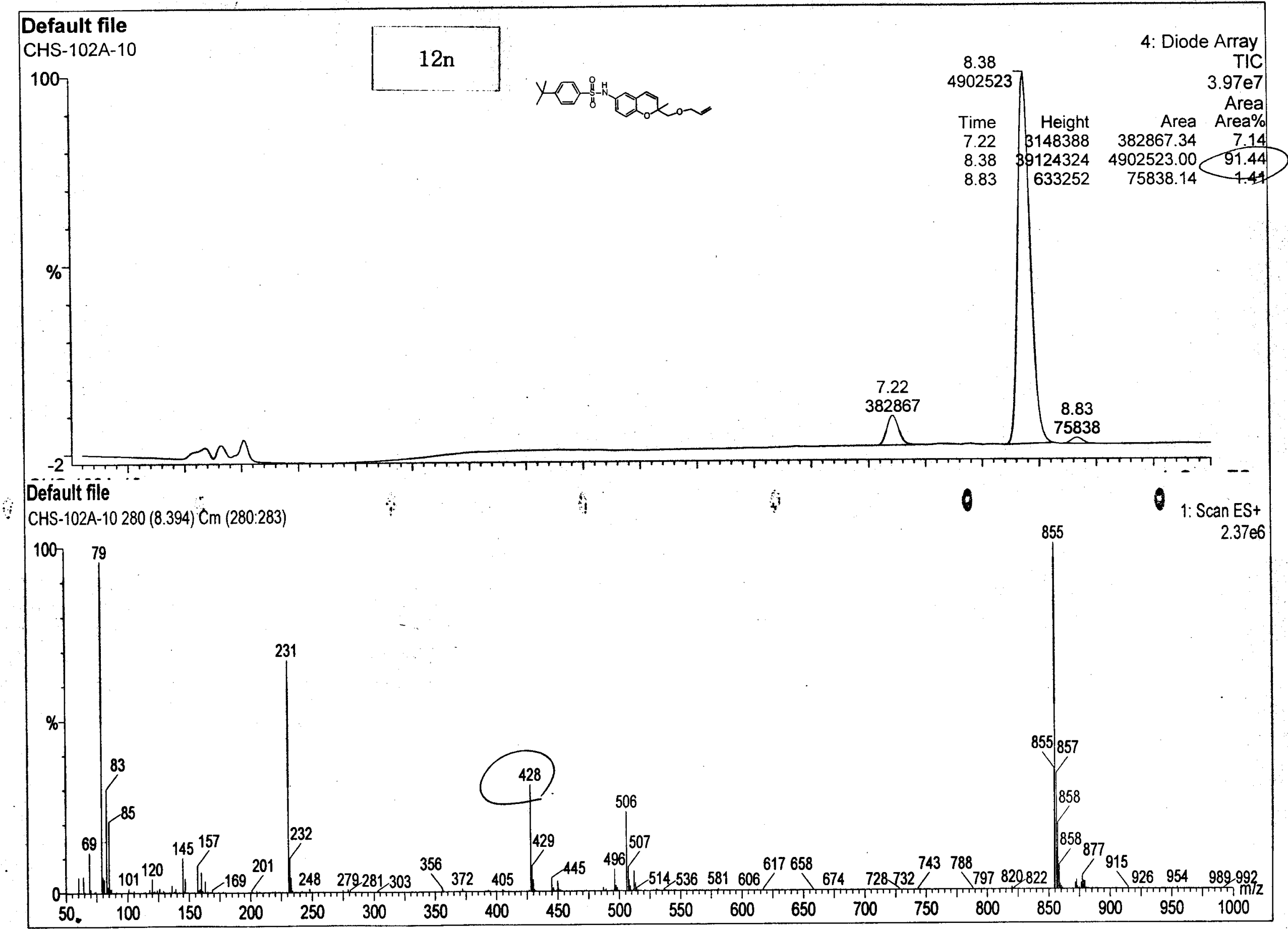



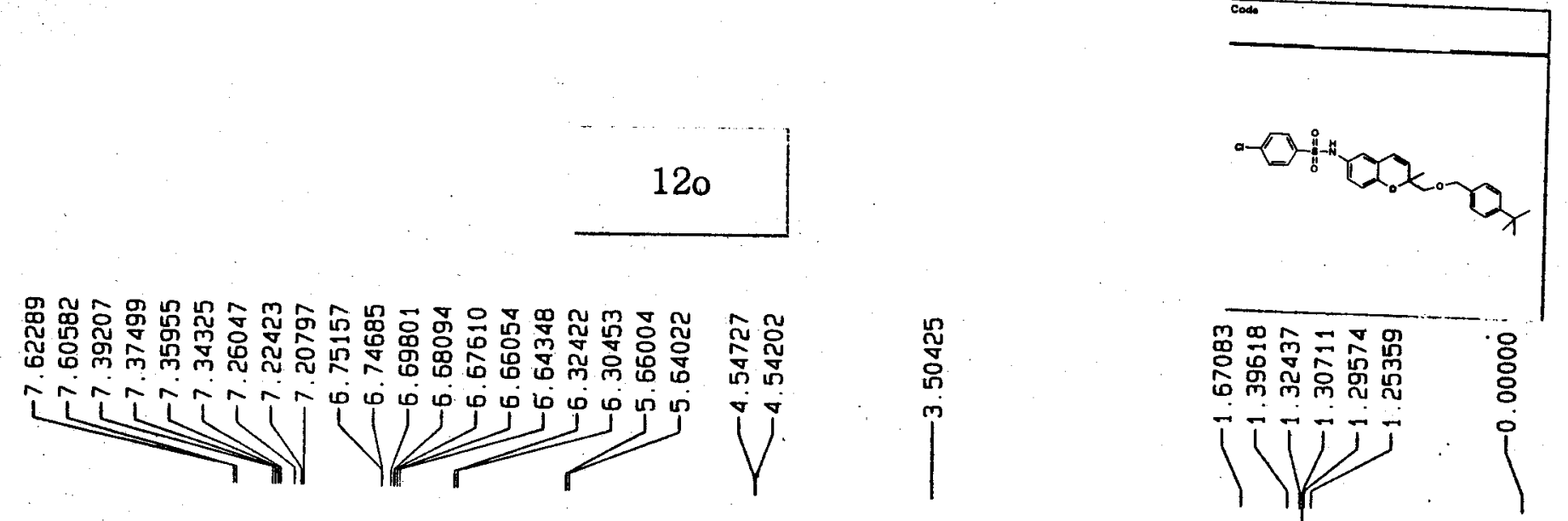

Current Data Parameters NAME chs-b102B-1

EXPNO

PAOCNO

F2 - Acquisition Parameters

Date

20041116

Time 22.13

INSTRUM spect PROBHD $5 \mathrm{~mm}$ DUL $13 C-1$

PULPROG

SOLVENT $\quad 65536$

NS $\operatorname{COCl3}$

DS

SWH $\quad 00$

FIDRES $\quad 0.114555$ H2

$40 \quad 0.114555 \mathrm{~Hz}$.

AG 4.3648143 SeC

RG 203.2

OW $\quad 66.600$ usec

$\begin{array}{ll}\mathrm{DE} & 6.00 \mathrm{usec} \\ \mathrm{TE} & 0.0 \mathrm{~K}\end{array}$

D1 $1.00000000 \mathrm{sec}$

MCREST $\quad 0.00000000 \mathrm{sec}$

MCWAK $\quad 0.01500000 \mathrm{sec}$

$z==z=s==$ CHANNEL I1 $z= \pm=z=z$ NUC

P1

$=x=2=2$

PL1 10.00 use

SF01 $\quad 500.1332508 \mathrm{MHZ}$

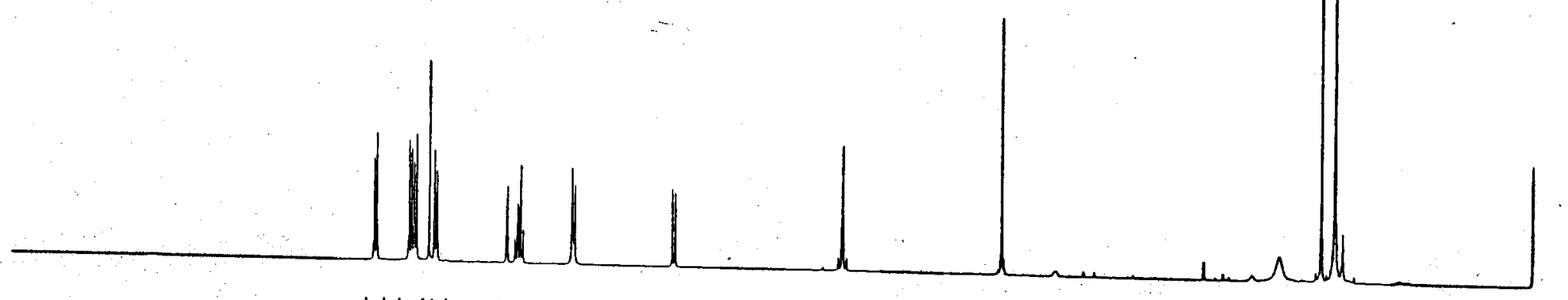

F2 - Processing par ameters

5I. 32768

SF $\quad 500.1300139 \mathrm{MHZ}$

HOW

SSB

$\begin{array}{lc}\text { LB } & 0 \\ \text { LB } & 0.30 \mathrm{HZ}\end{array}$

PC

1.00

10 NMR plot Darameters

$\begin{array}{ll}\mathrm{CX} & 20.00 \mathrm{~cm} \\ \mathrm{CY} & 20.00 \mathrm{~cm}\end{array}$

$\begin{array}{ll}\text { CY } & 20.00 \mathrm{~cm} \\ \text { FIP } & 10.000 \mathrm{oOm}\end{array}$

$\begin{array}{lr}\text { F1 } & 10.000 \mathrm{DDm} \\ \mathrm{FZ} & 5001.30 \mathrm{~Hz}\end{array}$

$\begin{array}{lr}F 2 P . & 5001.30 \mathrm{~Hz} \\ & 0.000 \mathrm{DD}\end{array}$

$\begin{array}{ll}\mathrm{F2} & 0.00 \mathrm{~Hz}\end{array}$

$\begin{array}{lr}\text { PPMCM } & 0.50000 \mathrm{pDm} / \mathrm{Cm} \\ \mathrm{HZCM} & 250.05500 \mathrm{HZ} / \mathrm{Cm}\end{array}$

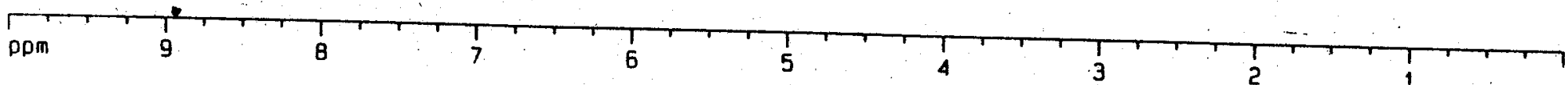




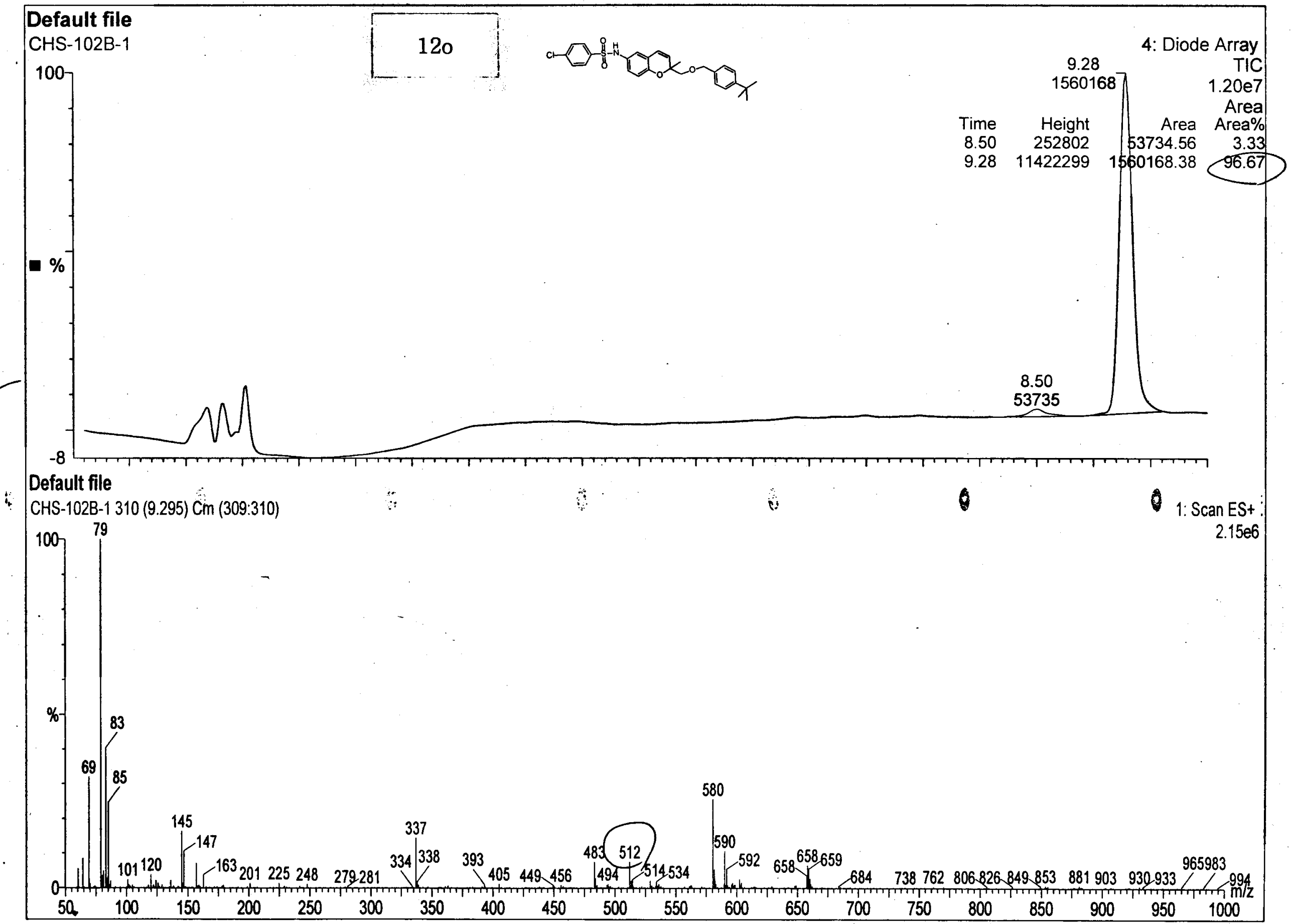




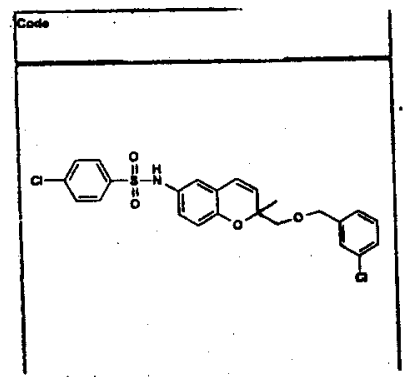

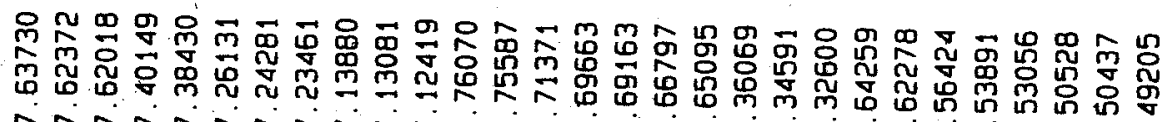
inisisi
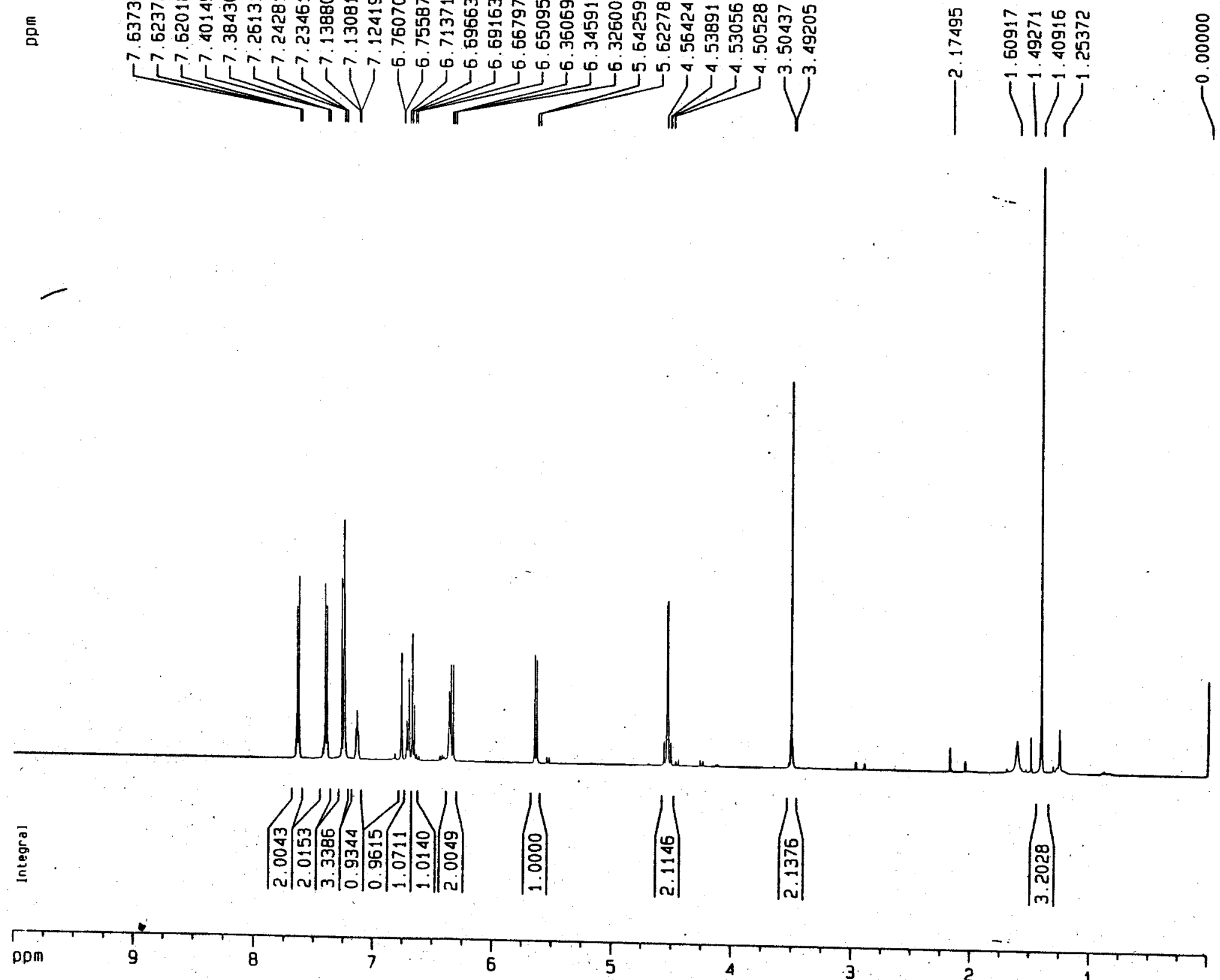

Current Data Parameters

PROCNO

F2 - Acquisition Parameters

20041116

rime 22.22

spect

PROBHD, $5 \mathrm{~mm}$ DUL $13 \mathrm{C}-1$

$\begin{array}{lr}\text { PULPROG } & 29 \\ \text { TD } & 65535\end{array}$

SOLVENT

NS

SWH $\quad 00$

FIDRES $\quad 0.114555 \mathrm{~Hz}$

$\mathrm{AQ} \quad 4.3648143 \mathrm{sec}$

RG $\quad 181$

OW 66.600 usec

DE 6.00 use

TE $0.0 \mathrm{~K}$

$\quad 1.00000000 \mathrm{sec}$

MCREST $\quad 0.00000000 \mathrm{sec}$

MCWRK $\quad 0.01500000 \mathrm{sec}$

$===== \pm=$ CHANNEL if

NUC1

10.00 usec

SF01 $\quad-2.00 \mathrm{~dB}$

F2 - Processing parameters

SI 32768

SF $\quad 500.1300135 \mathrm{MHZ}$

WDW

$\begin{array}{lc}\text { LB } & 0 \\ G B & 0.30 \mathrm{~Hz}\end{array}$

PC

10 NMA plot parameters

CX. $\quad 20.00 \mathrm{~cm}$

$\begin{array}{ll}\mathrm{CY} & 10.00 \mathrm{~cm}\end{array}$

FIP $\quad 10.000 \mathrm{ppm}$

$F 1 \quad 5001.30 \mathrm{~Hz}$

F2P $\quad 0.000 \mathrm{pDm}$

$\begin{array}{ll}\mathrm{F2} & 0.00 \mathrm{~Hz}\end{array}$

$\begin{array}{lr}\text { PPMCM } & 0.50000 \mathrm{pDm} / \mathrm{cm} \\ \mathrm{HZCM} & 250.06500 \mathrm{~Hz} / \mathrm{cm}\end{array}$ 


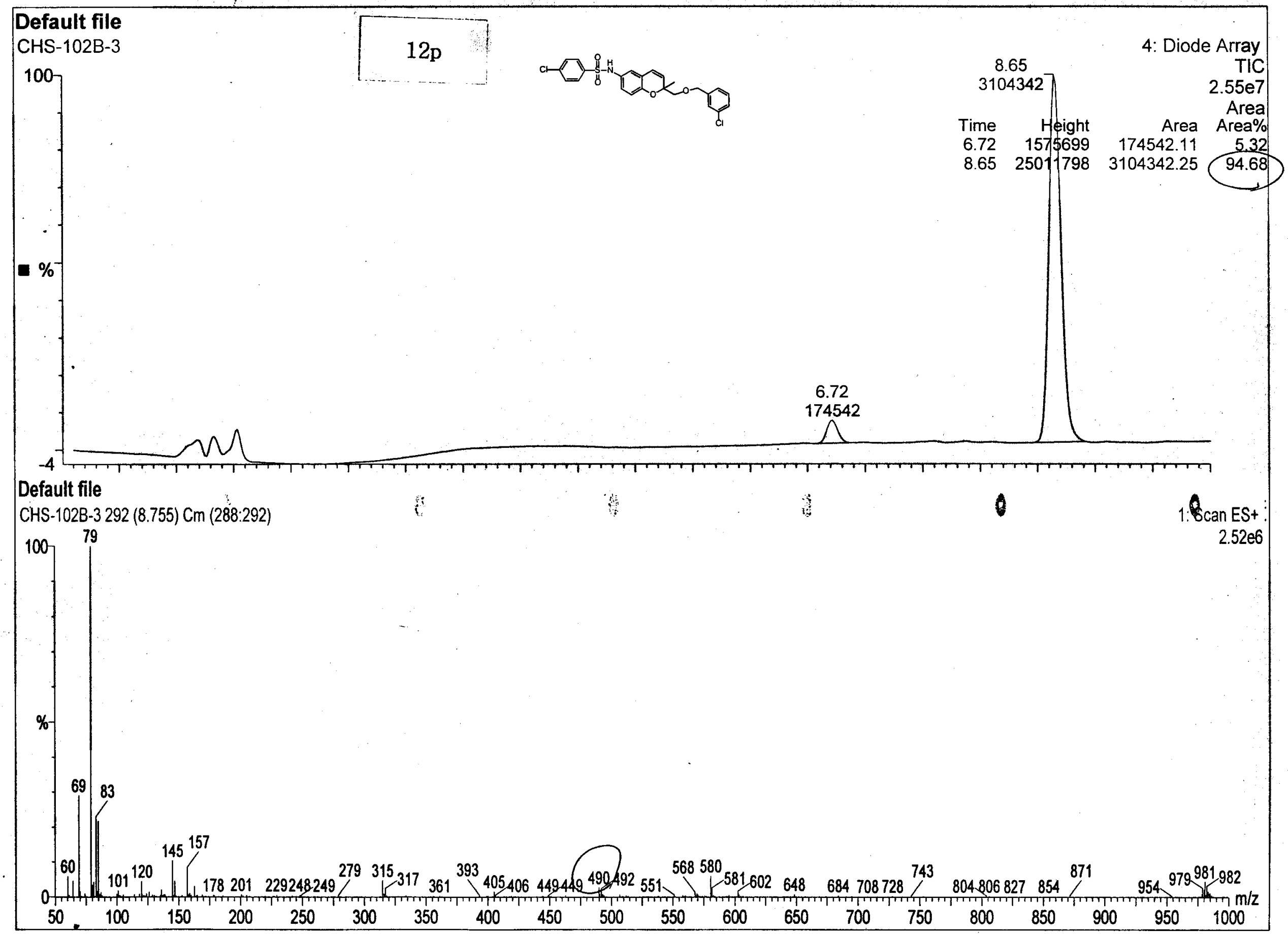




\section{$12 \mathrm{q}$}

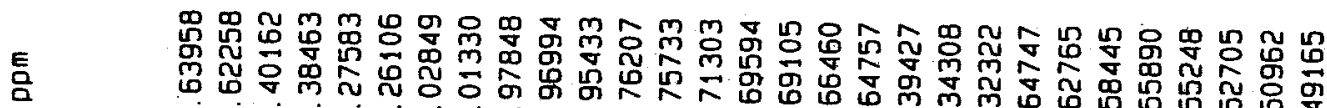

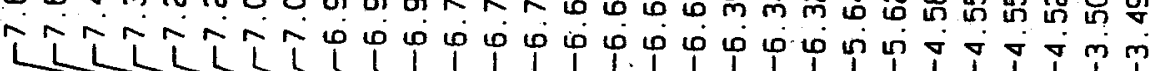
11

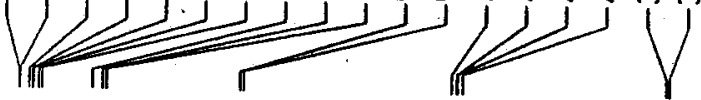

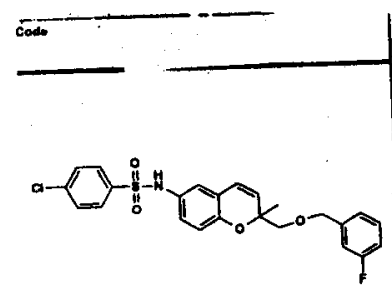
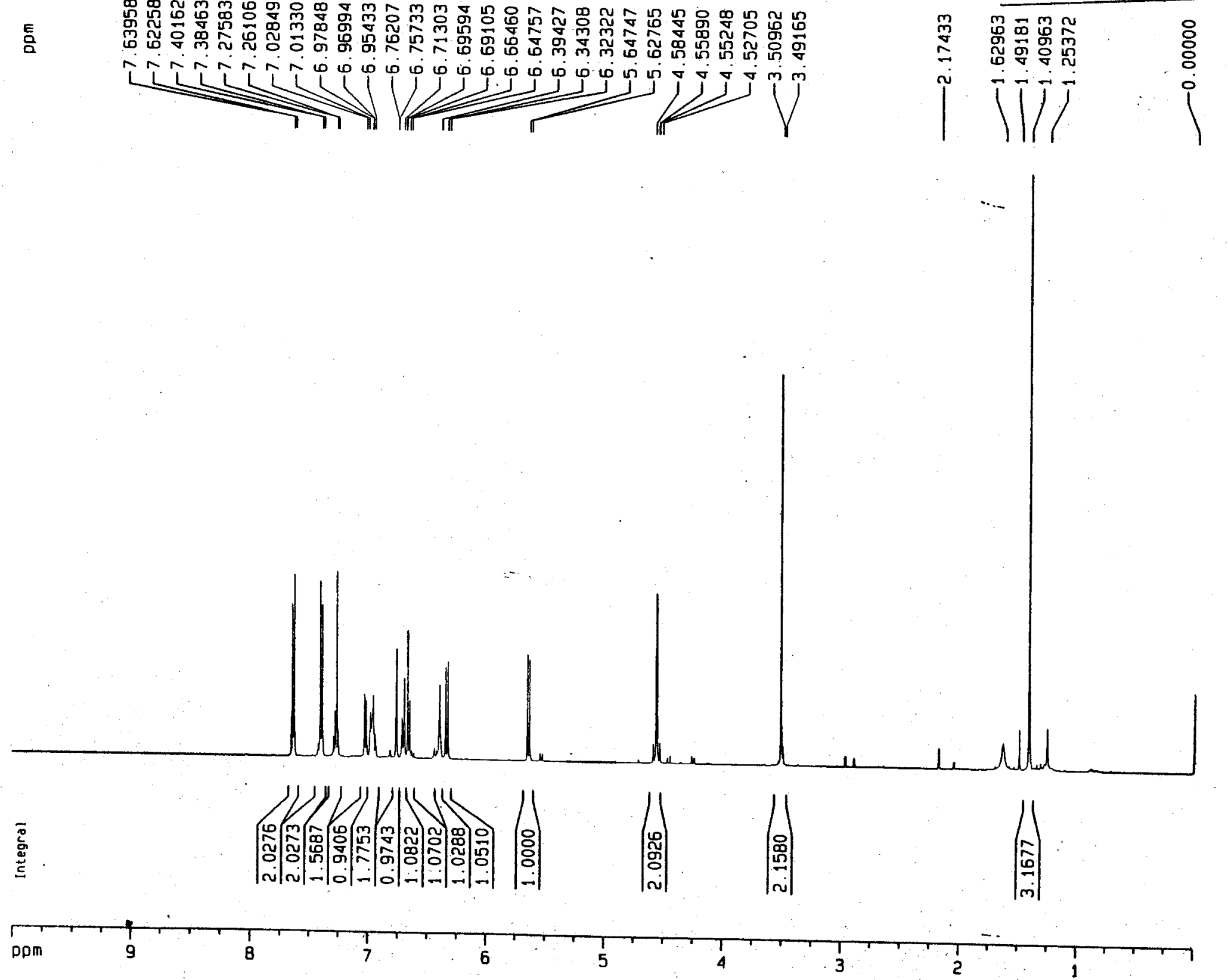

PROCNO

F2 - Acquisition Parameters

Date_ 20041116

$\begin{array}{lr}\text { Iime } & 22.33\end{array}$

INSTRUM Spect

PROBHD $5 \mathrm{~mm}$ DUL $13 \mathrm{C}-1$

PULPROG

TO

SOLVENT

NS

DS

SWH

FIDRES $\quad 7507.507 \mathrm{~Hz}$

4. $3648914 \mathrm{~Hz}$

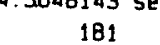

181

66.600 usec
6.00 usec

0.00 use

TE $\quad 0.0 \mathrm{~K}$

$\quad 1.00000000 \mathrm{sec}$

MCREST $\quad 0.00000000 \mathrm{SEC}$

MCHK $\quad 0.01500000 \mathrm{sec}$

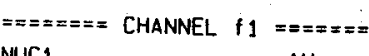

NUC1

$P_{1} \quad 10.00$ usec

PL1 $\quad-2.00 \mathrm{~dB}$

$500.133250 \mathrm{BHz}$

F2 - Processing parameters

SI 32768

SF $\quad 500.1300137 \mathrm{MHZ}$

WDW

SSB

LB

GB

EM

$0.30 \mathrm{~Hz}$

0

1.00

DMA plot parameters

CX $20.00 \mathrm{~cm}$

Cr $\quad 10.00 \mathrm{~cm}$

FIP $\quad 10.000 \mathrm{DPm}$

F1 $\quad 5001.30 \mathrm{~Hz}$

F2P. $\quad 0.000 \mathrm{pOm}$

F2. $0.00 \mathrm{~Hz}$

PPMICM $\quad 0.50000 \mathrm{pDm} / \mathrm{Cm}$

$\mathrm{HZCM} \quad 250.06500 \mathrm{~Hz} / \mathrm{cm}$ 


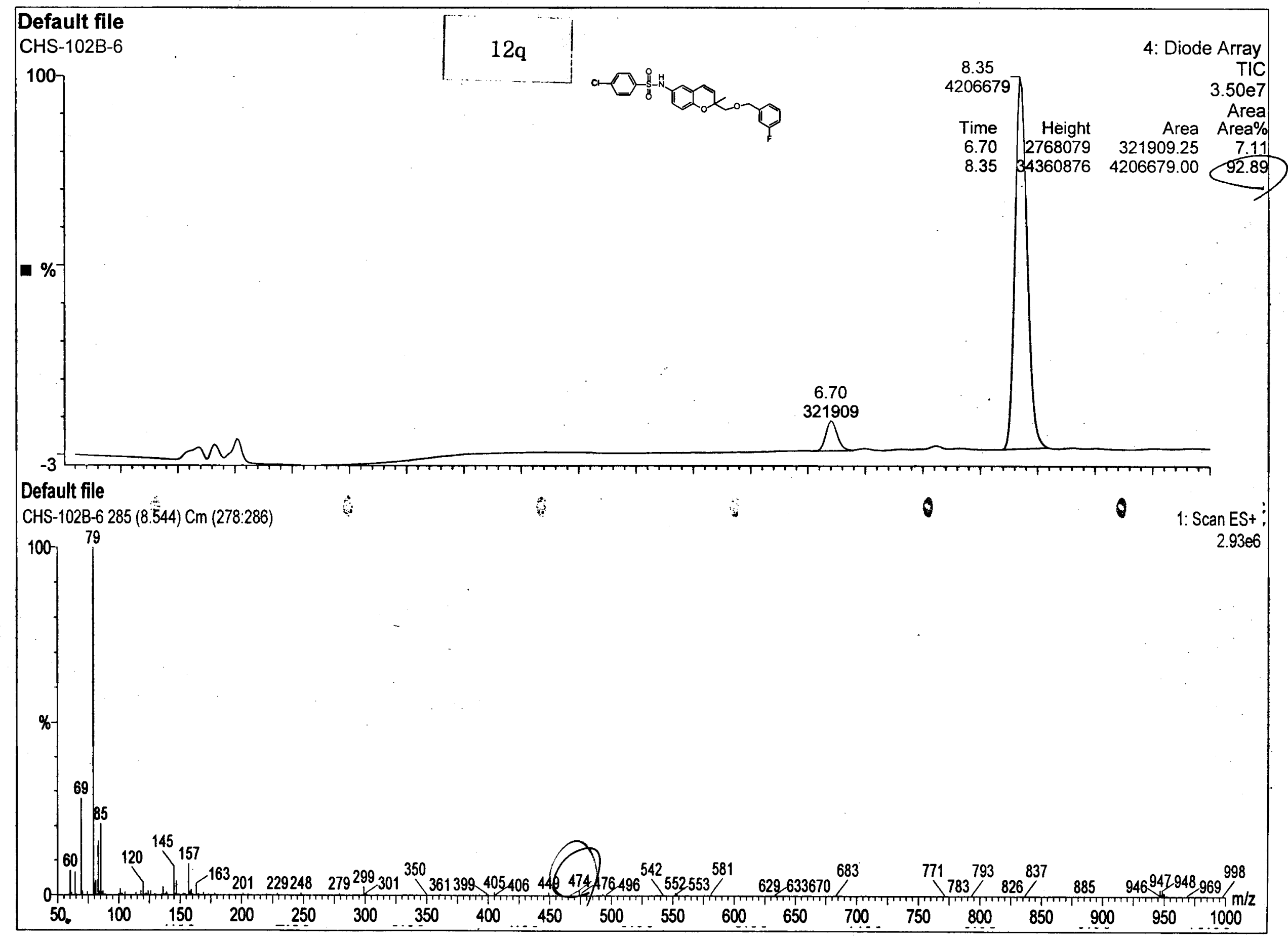




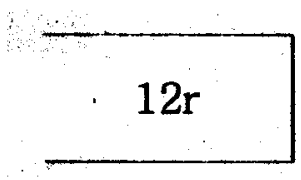

⿷匚⿱⿰㇒一大口

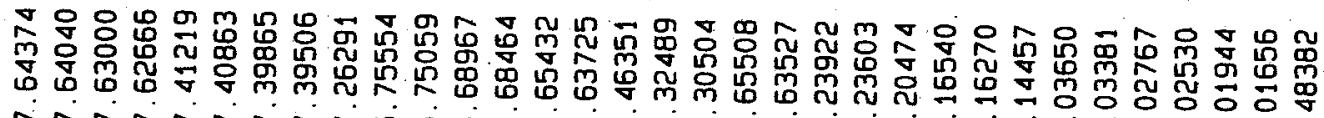

L
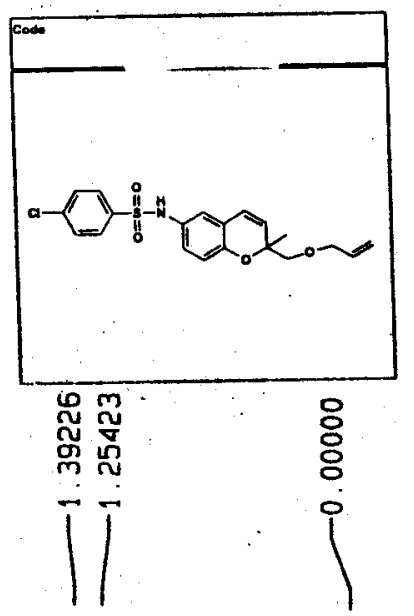

$\mid$
Curcent Data Parameters NAME EXPNO PROCNO

F2 - Acquisition Parameters Date_ 20041116 Time $\begin{array}{ll}\text { INSTRUM } & 22.46 \\ \text { spect }\end{array}$ PROBHD 5 mm DUL $13 \mathrm{C}-1$ PULPROG 29

29
65536

$\begin{array}{ll}\text { TD } & 65536 \\ \text { SOLVENT } & \text { CDC13 }\end{array}$

NS

DS
SWH
FIDRES

AQ

AG

DW

DE

TE

D1

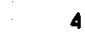

$7507.507 \mathrm{~Hz}$ $0.114555 \mathrm{~Hz}$

4. $3648143 \mathrm{sec}$$$
181
$$

66.600 usec 6.00 usec $6.00 \mathrm{usec}$
$0.0 \mathrm{~K}$

$1.00000000 \mathrm{sec}$

$1.00000000 \mathrm{sec}$ $0.01500000 \mathrm{sec}$ $0.01500000 \mathrm{sec}$

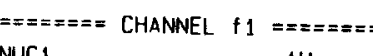
NUC1

P1

PL1 10.00 usec

SFD1 $500 \quad-2.00 \mathrm{~dB}$

F2 - Processing parameters SI 32768 SF $\quad 500.1300124 \mathrm{MHZ}$

WOW

SSB

LB PC 500.1300124 $E M$
0 $0.30 \mathrm{~Hz}$ 0
1.00

10 NMR plot parameters CX $20.00 \mathrm{~cm}$ $\mathrm{Cr} \quad 10.00 \mathrm{~cm}$ FIP $\quad 10.000 \mathrm{pDm}$ $\begin{array}{ll}\text { F1 } & 5001.30 \mathrm{~Hz} \\ \mathrm{~F} 2 \mathrm{P} & 0.000 \mathrm{pgm}\end{array}$ F2 $0.00 \mathrm{~Hz}$ PPMCM $\quad 0.50000 \mathrm{pDm} / \mathrm{Cm}$

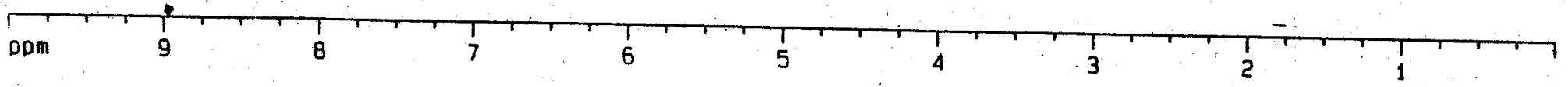




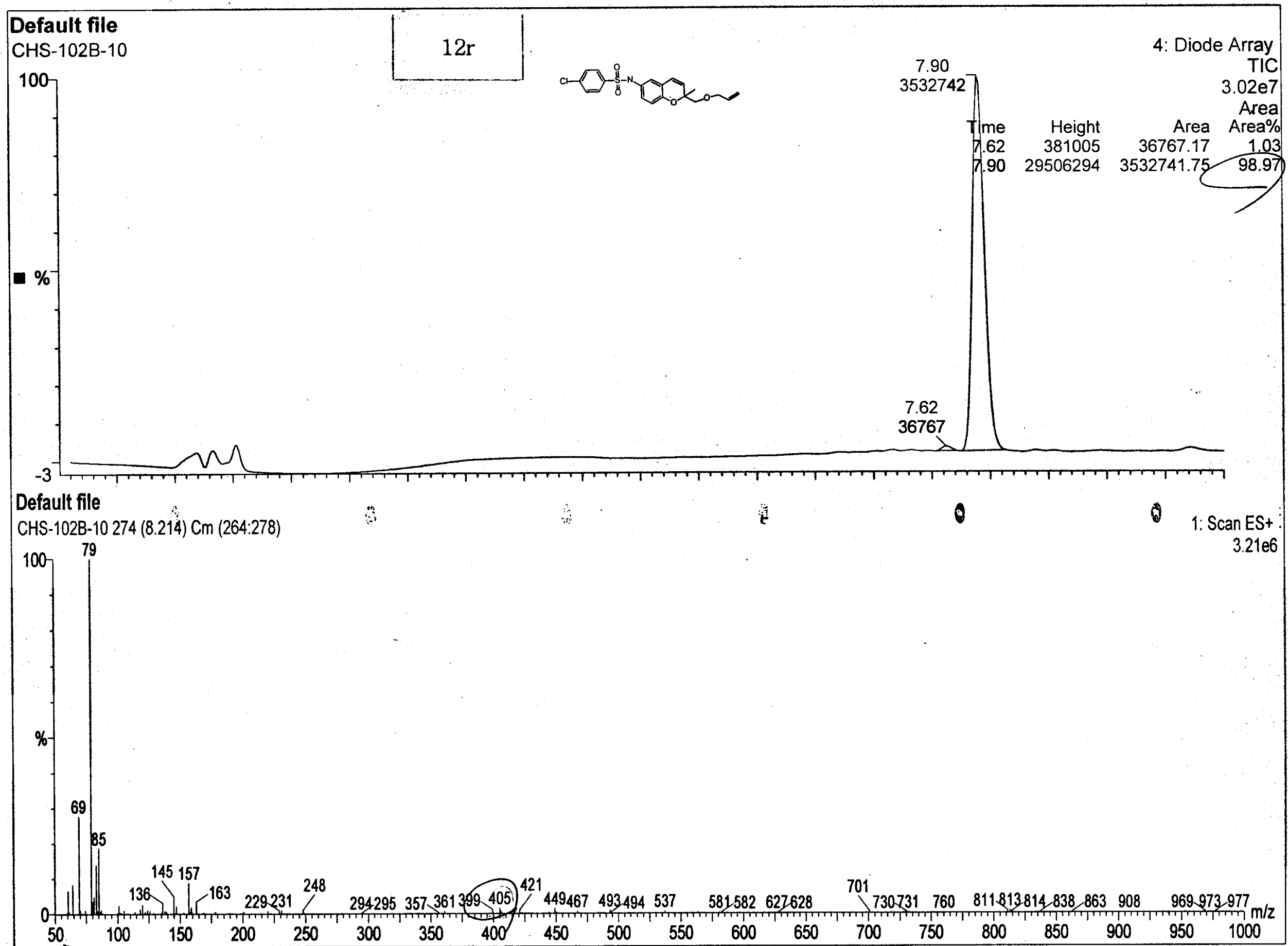




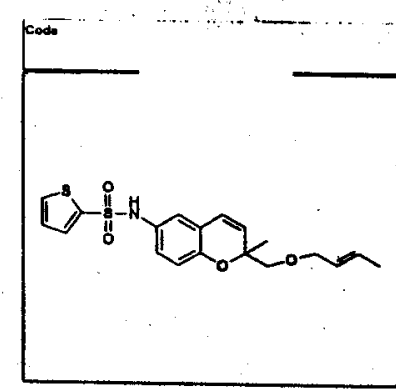

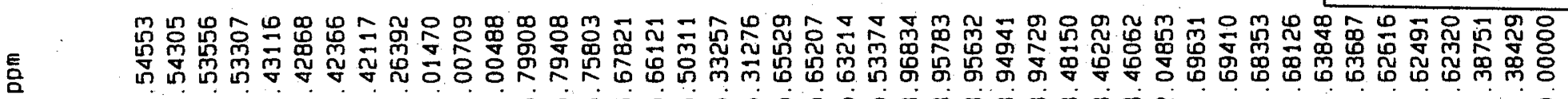
NAME

Current Data Parameters NAME chs-b106A-9 EXPNO $\longrightarrow$
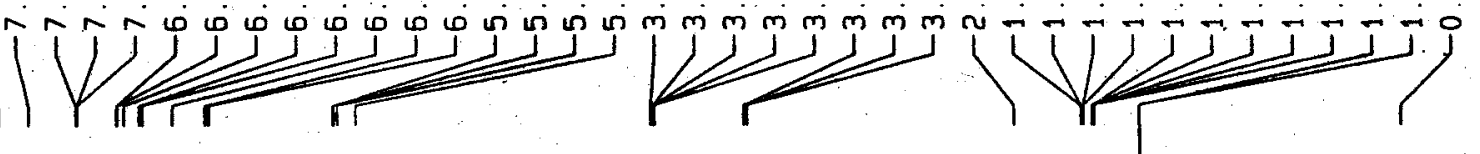

F2 -

Date_-
Time
INSTAU

INSTRUM

PROBHO
PULPROG PULPADG

TO

NS

NS

DS

FIDRES

$A 0$

RG

DW

DE

TE

D1

MCWAK

Chs-b106A-9

Quisition Parameters

20041123

18.49

spect

DUL $13 C-1$

29
65536

$\operatorname{COC} 13$

4

$7507.507 \mathrm{~Hz}$

$0.114555 \mathrm{~Hz}$
$4.3648143 \mathrm{ser}$

4. $3648143 \mathrm{se}$

114

66.600 usec
6.00 usec $0.0 \mathrm{~K}$

$1.00000000 \mathrm{sec}$

$0.00000000 \mathrm{sec}$

$0.01500000 \mathrm{sec}$

$=z=z x=z$ CHANNEL $f 1=x=z=s=$

NUC1 $1 \mathrm{H}$

$P_{1} \quad 10.00$ usec

PL1 $\quad-2.00$ dB

SFO1 500.1332508 MHZ

F2 - Processing parameters

SI 32768

SF $\quad 500.1300119 \mathrm{MHz}$

WOW EM

SSB

GB

GB

0
$30 \mathrm{~Hz}$

0
0
1.00

10 NMR plot parameters

CX $\quad 20.00 \mathrm{~cm}$ $\begin{array}{ll}\mathrm{CX} & 20.00 \mathrm{~cm} \\ \mathrm{Cr} & 12.00 \mathrm{~cm}\end{array}$ F1P $\quad 10.000 \mathrm{ppm}$ F1 $\quad 5001.30 \mathrm{~Hz}$ F2P $\quad-0.500 \mathrm{DDm}$ $\begin{array}{ll}F 2 P & -0.500 \mathrm{DPH} \\ F 2 & -250.06 \mathrm{~Hz}\end{array}$

PPMCM $\quad 0.52500 \mathrm{ppm} / \mathrm{cm}$

$\mathrm{HZCM} \quad 262.56824 \mathrm{~Hz} / \mathrm{cm}$ 


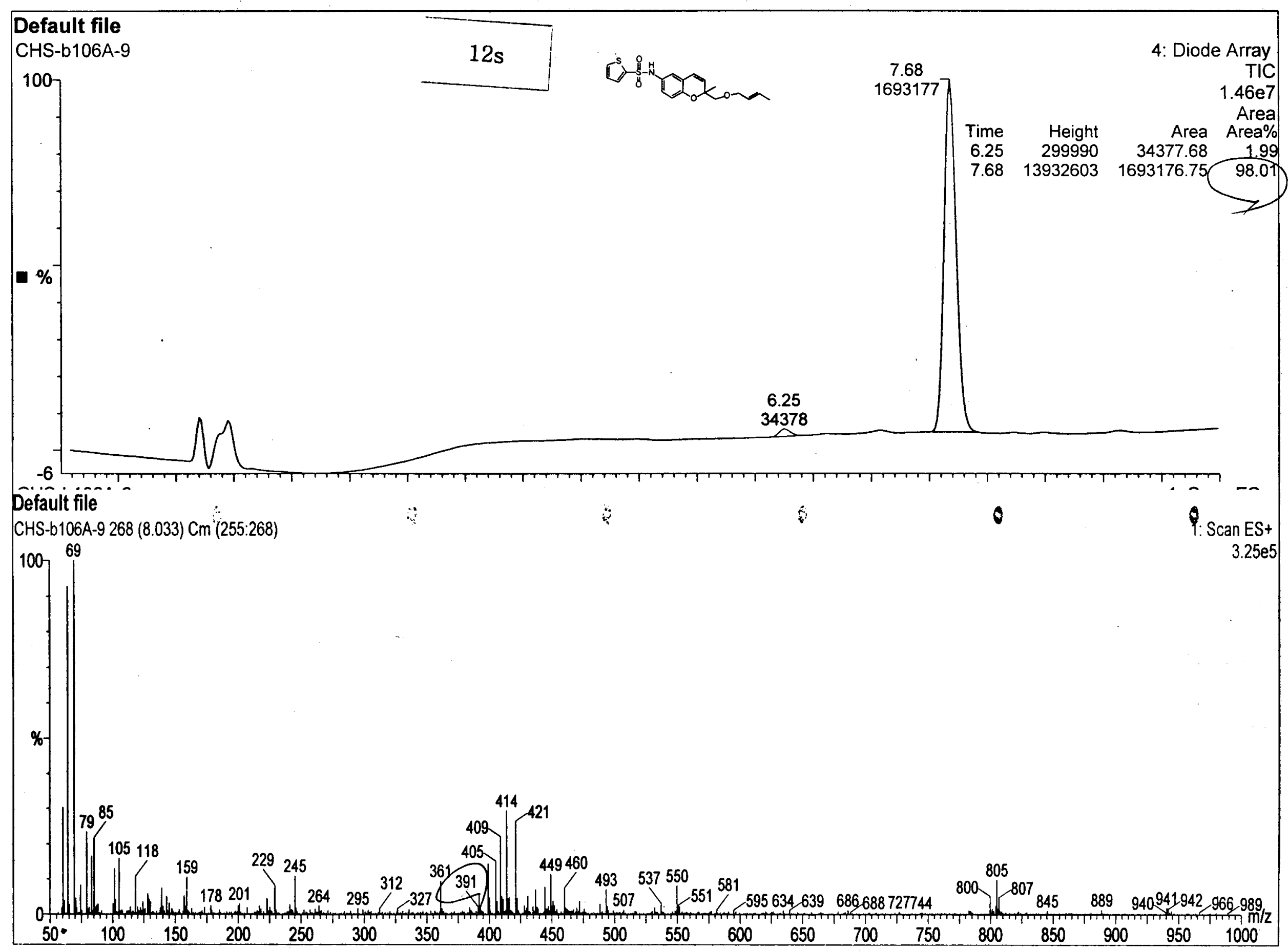




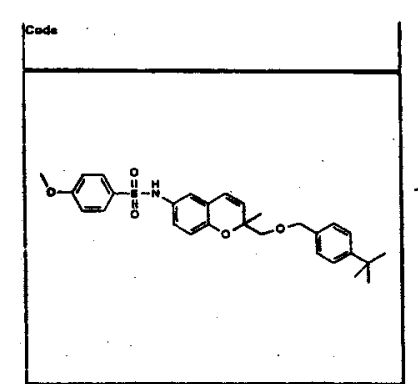

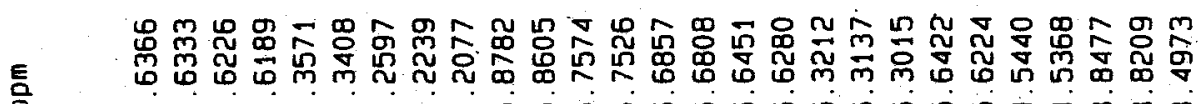
r

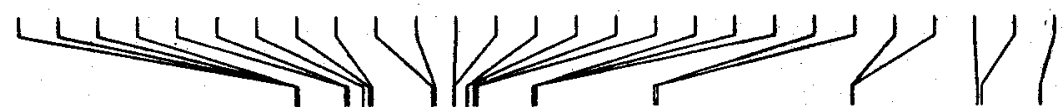

8

chs-b106B-1

EXPNO

1

F2 - Acquisition Parameters

Date 20041123

Date _ 20041123

Time $\quad 20.07$

PROBHD $5 \mathrm{~mm}$ DUL $13 \mathrm{C}-1$

PROBHD $5 \mathrm{~mm}$ DUL $13 \mathrm{C}-1$

$\begin{array}{lr}\text { PULPROG } & 29 \\ \text { TD } & 65536\end{array}$

SOLVENT CDC13

NS

OS

SHH $\quad 7507.507 \mathrm{~Hz}$

FIDRES $\quad 0.114555 \mathrm{~Hz}$

AQ $4.3648143 \mathrm{sec}$

AQ 4.3648143

AW $\quad 66.600$ usec

$\begin{array}{lr}66.600 \text { usec } \\ \text { DE } & 6.00 \text { usec }\end{array}$

: $0.0 \mathrm{~K}$.

$\quad 1.00000000 \mathrm{sec}$

MCREST $\quad 0.00000000 \mathrm{sec}$

MCWRK $\quad 0.01500000 \mathrm{sec}$

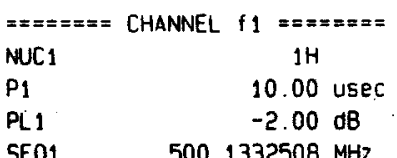

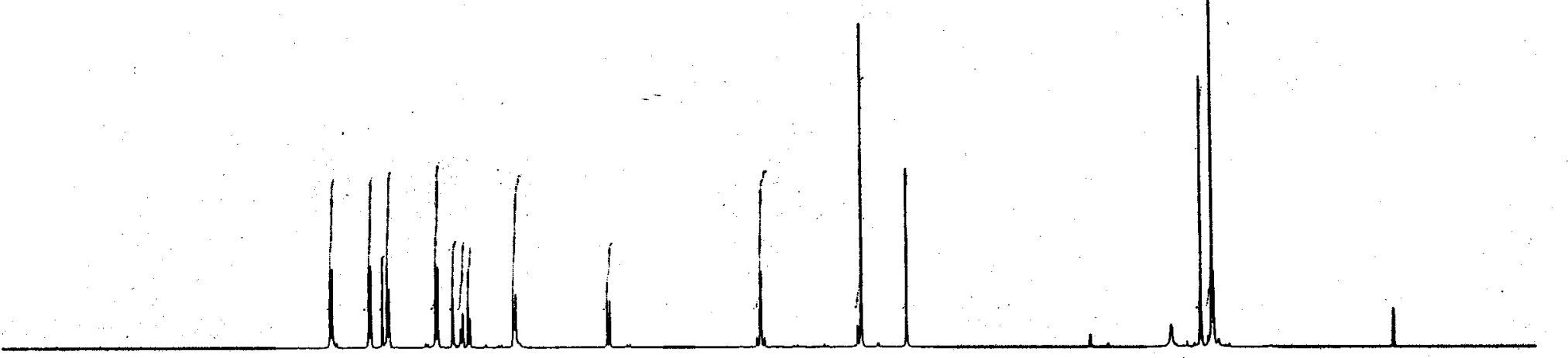

$500.1332508 \mathrm{MHz}$

F2 - Processing parameters

$\begin{array}{lc}\text { SI } & 32768 \\ \text { SF } & 500.1300143 \mathrm{MHz}\end{array}$

WOW

SSB
LB

LB

$P C$

EM

0. 0 $30 \mathrm{~Hz}$

0

10 NMR plot parameters

CX 20.00

$\mathrm{Cr} \quad 12.50 \mathrm{~cm}$

F1P $10.000 \mathrm{pPa}$

F1 $500130 \mathrm{~Hz}$

F2P $-1.030 \mathrm{PP}$

F2 $\quad-515.15 \mathrm{~Hz}$

PPACM

$0.55150 \mathrm{pom} / \mathrm{cm}$

\section{$\mathrm{S} 114$}

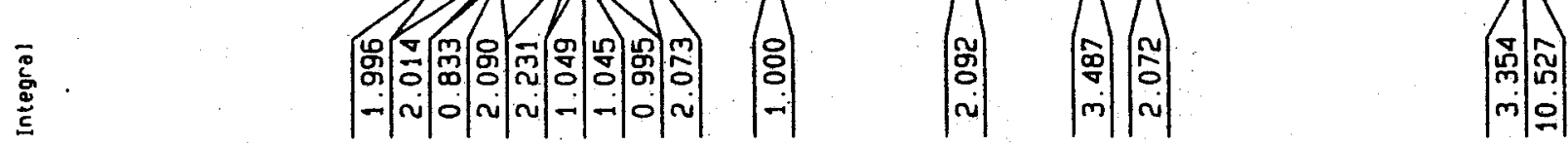

$275.82297 \mathrm{~Hz} / \mathrm{cm}$ 


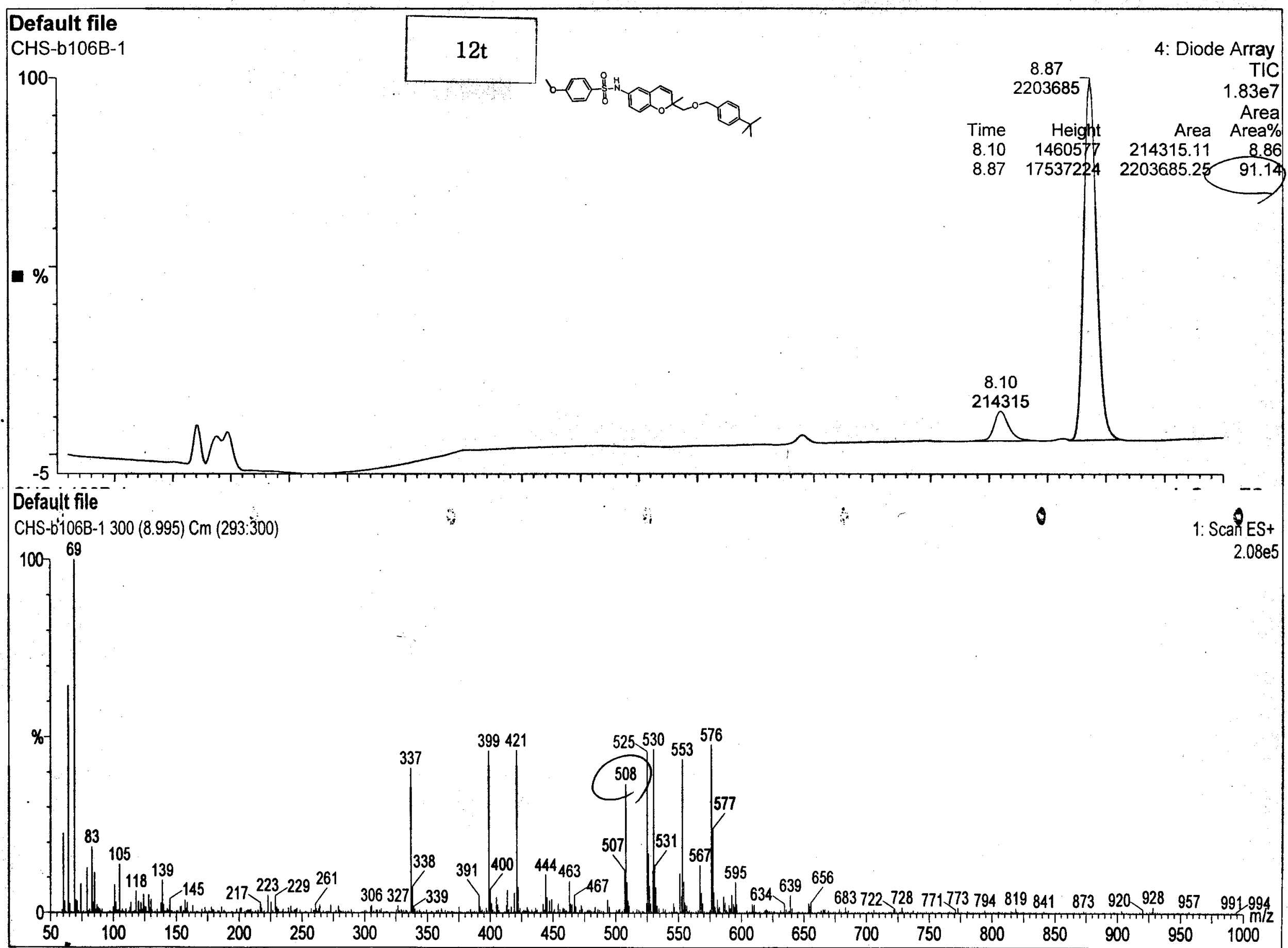




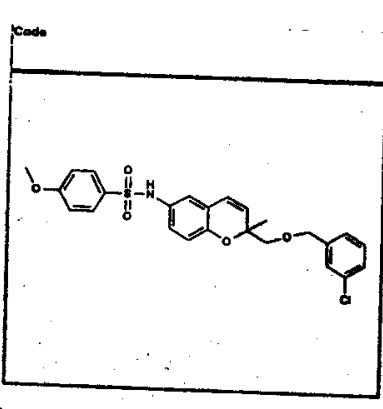

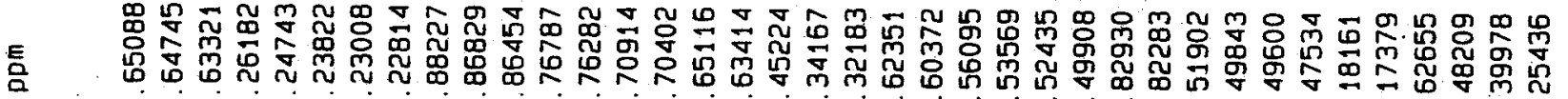

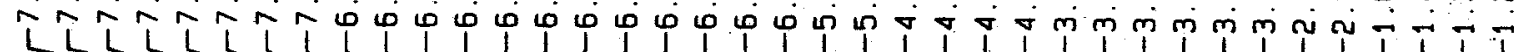
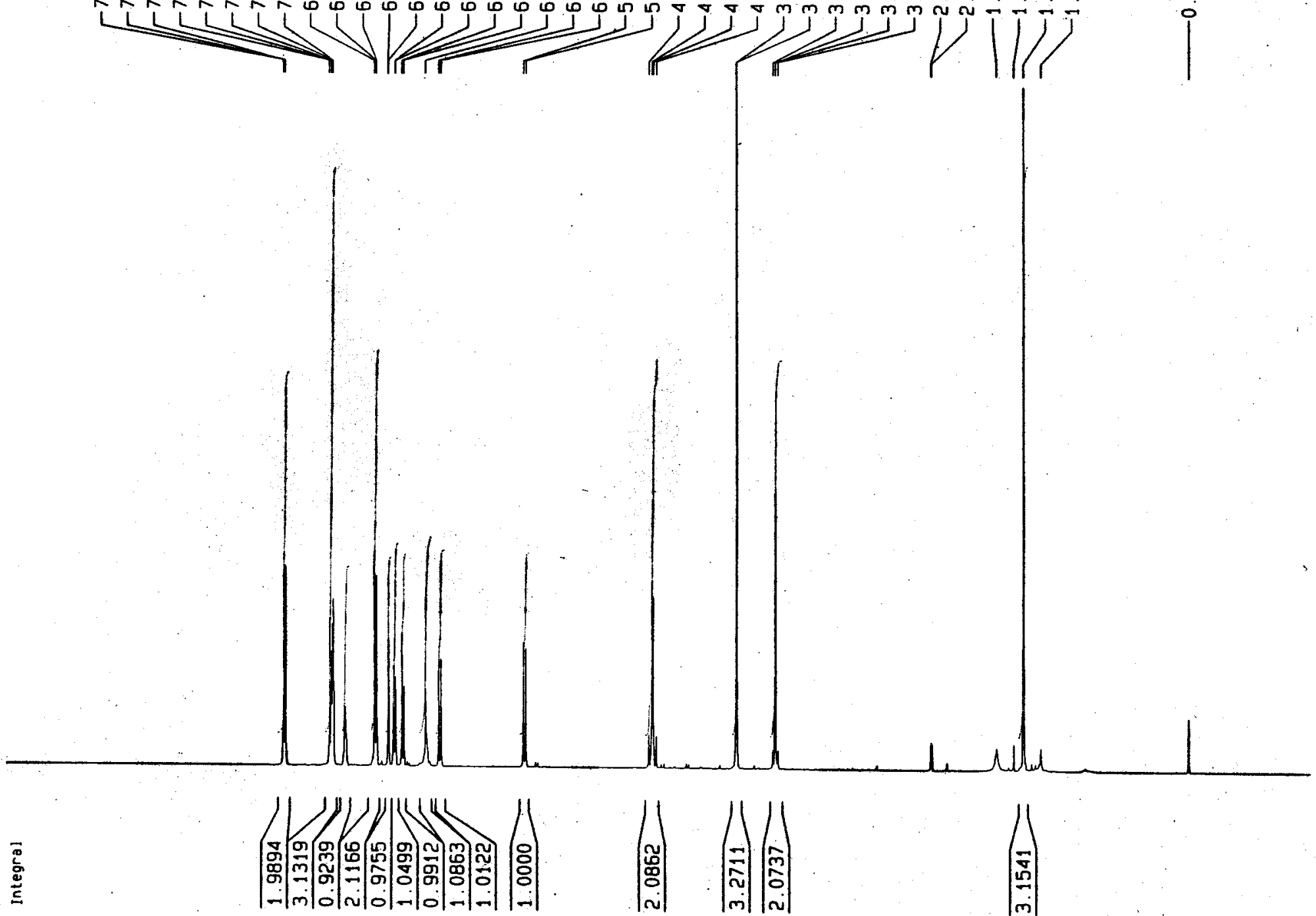

$\left|\begin{array}{c}n \\ -1\end{array}\right|$ 


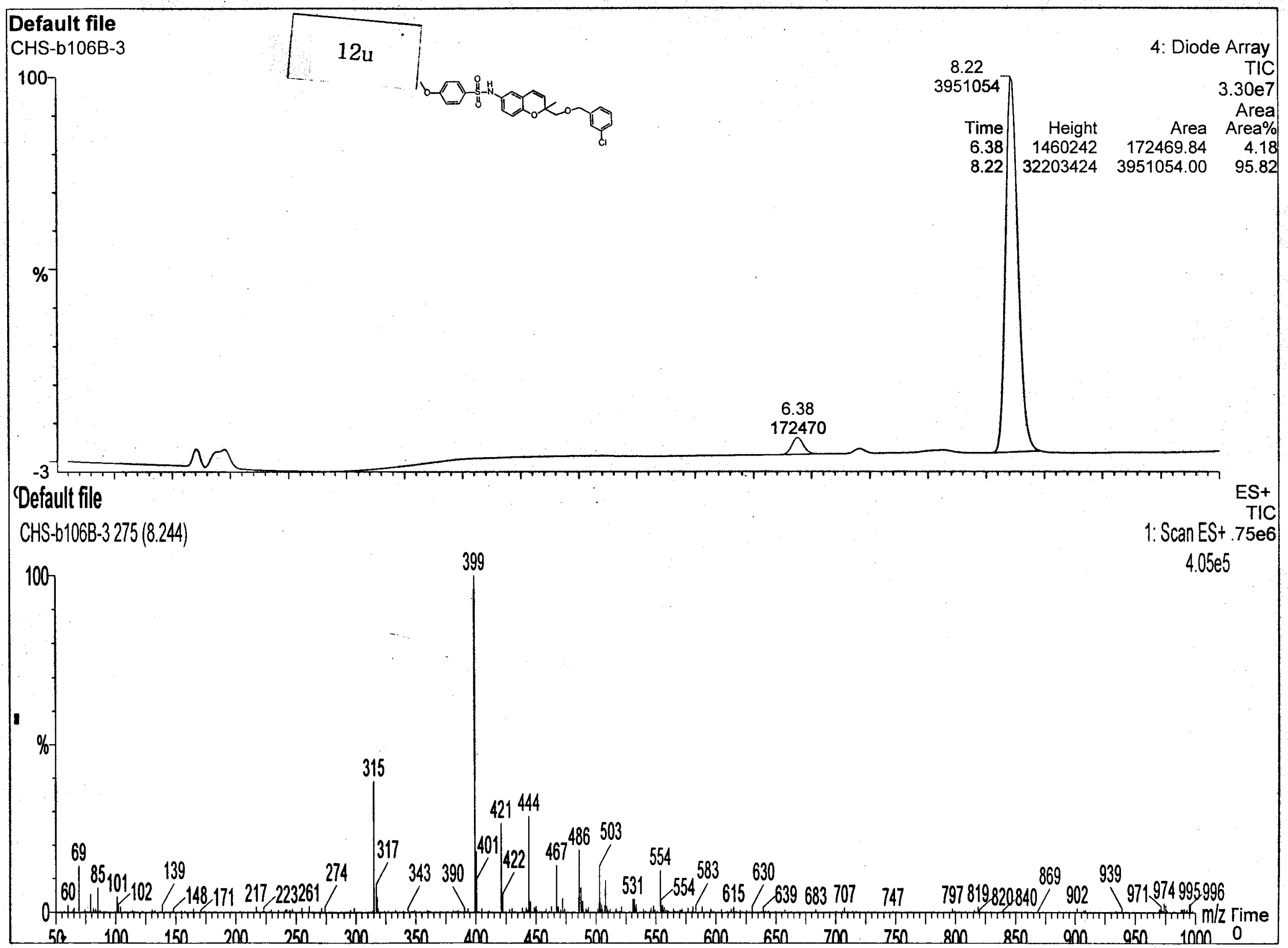




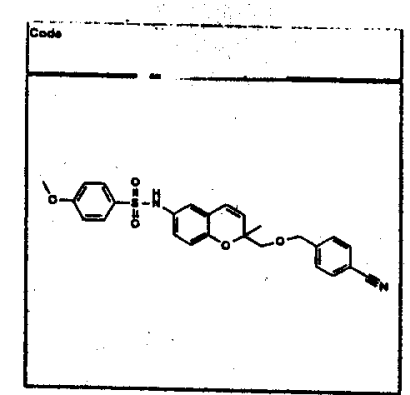

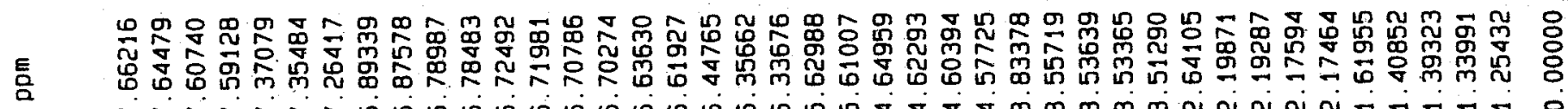

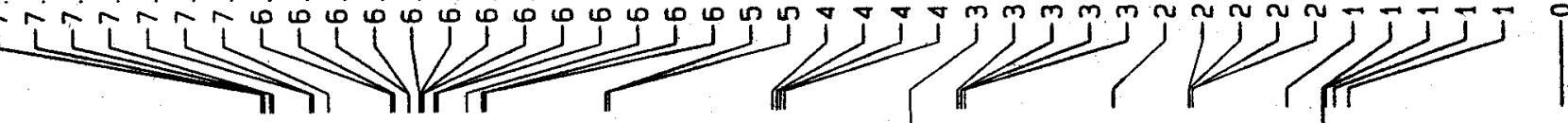

กา I 11

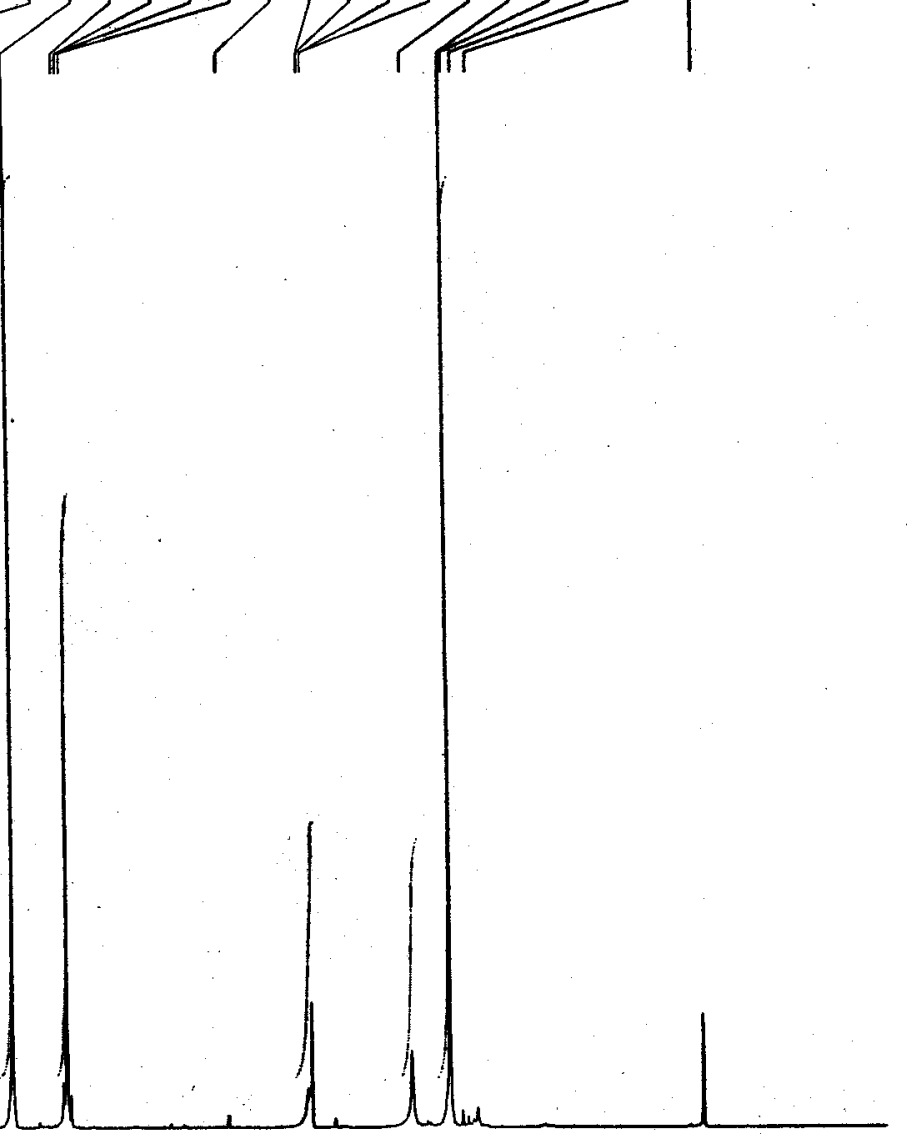

F2 - Acquisition Parameters

Date

Date

Time

.

PROBHD $5 \mathrm{~mm}$ DUL $13 \mathrm{C}-1$

PULPROG

TD

SOL VENT

NS

DS

SWH

FIDRES
$A Q$
AG

DW

DE

TE
D1

MCREST

MCWRK

29
65536

CDC 3
$==x=z z==$ CHANNEL fi $=z=z===$
NUC1 $1 \mathrm{H}$
P1 $\quad 10.00$ use

5001332500

F2 - Processing parameters

F2 - Processing parameters
SI
32768

$\begin{array}{lc}\text { SI } & 32768 \\ \text { SF } & 500.1300121 . \mathrm{MHZ}\end{array}$

WOW

$\begin{array}{lc}\text { SSB } & 0 \\ \text { LB } & 0.30 \mathrm{HI}\end{array}$

$\begin{array}{lr}6 B & 0.30 \mathrm{HR} \\ P C & 0\end{array}$

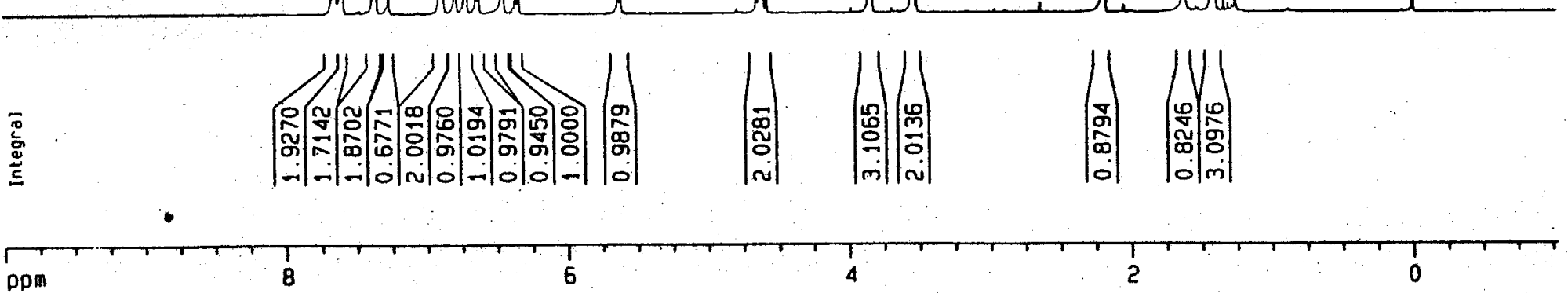

ID NMR plot parameters

$\begin{array}{lc}\text { CX } & 20.00 \mathrm{~cm} \\ \text { CY } & 12.50 \mathrm{~cm} \\ \text { F1P } & 10.000 \mathrm{pDm} \\ \text { F1 } & 5009.30 \mathrm{~Hz} \\ \text { F2P } & -1.030 \mathrm{pDm} \\ \text { FZ } & -515.16 \mathrm{~Hz} \\ \text { PPMCM } & 0.55150 \mathrm{pPm} / \mathrm{cm} \\ \text { HZCM } & 275.82297 \mathrm{~Hz} / \mathrm{cm}\end{array}$




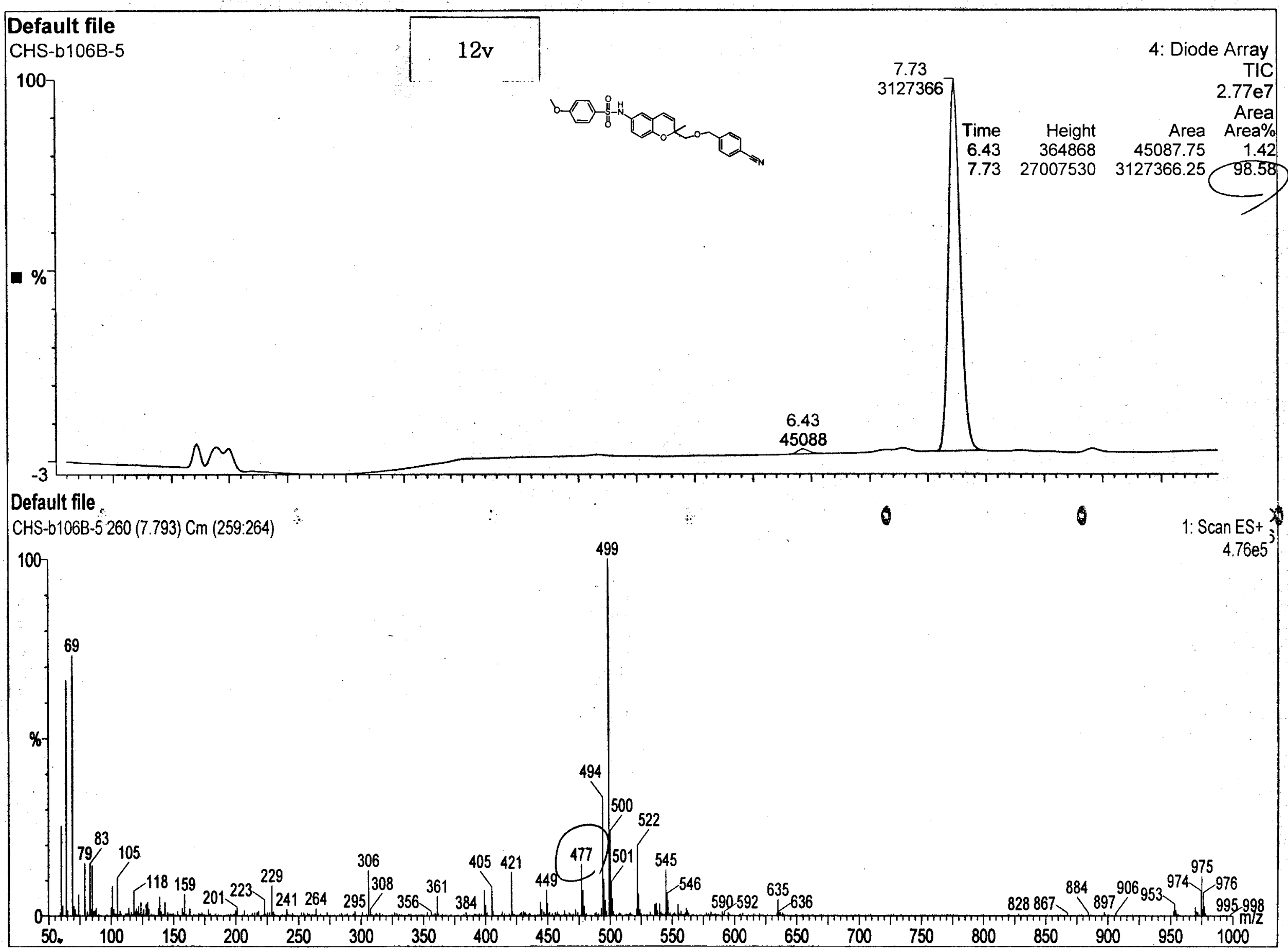




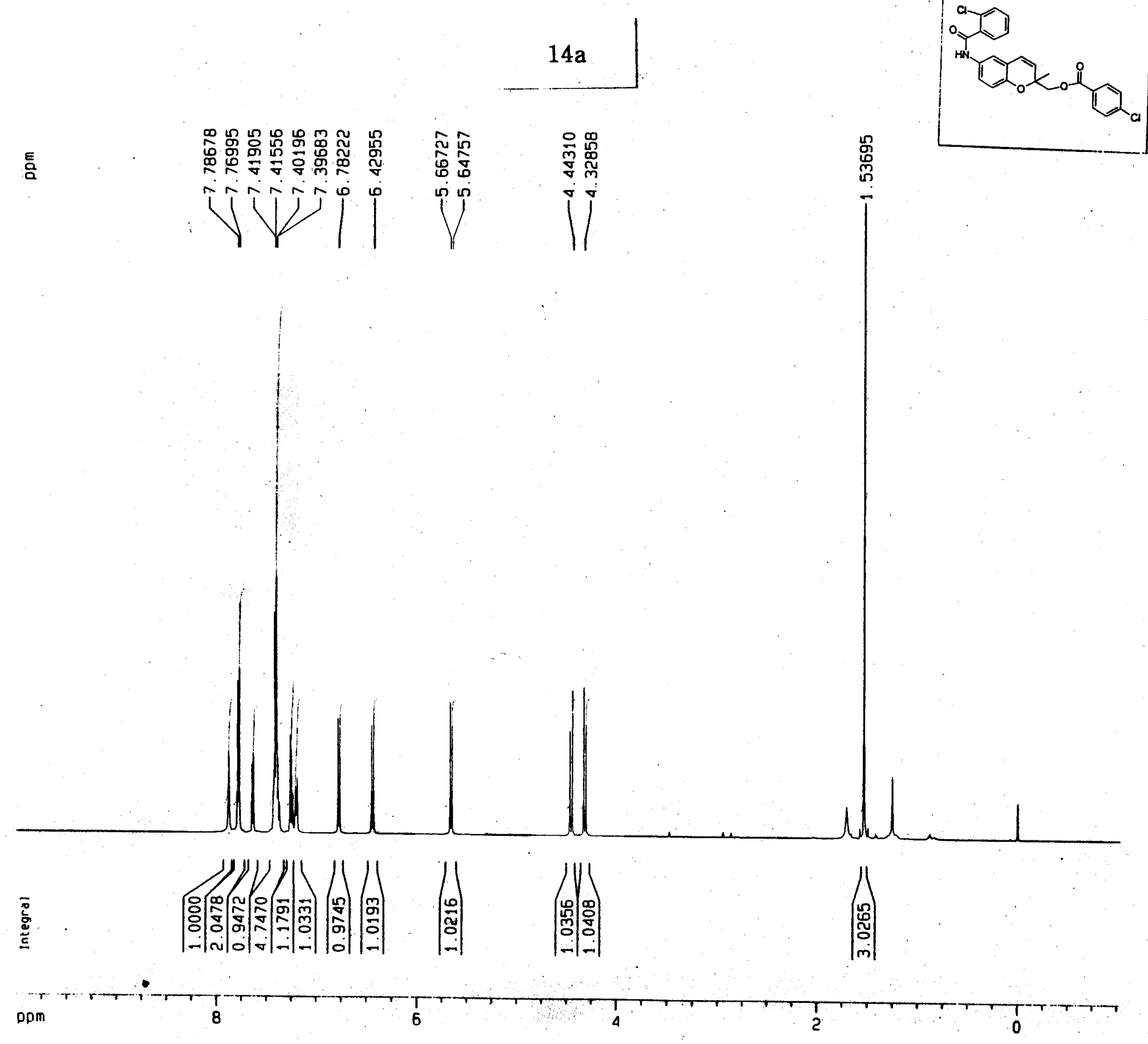

Current Data Parameters
NAME 


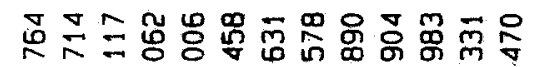

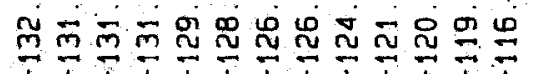

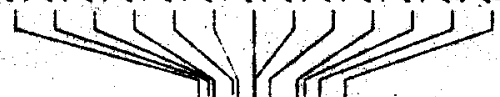

惫等哭总

조용

(1)

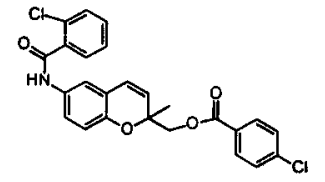

Current Data Parameters

NAME

JROCNO

chs-b57-3

용

=2 - Acquisition Parameters

Jate

I Ime

20050712

InSTRuM spect

PROBHO 5 mm OUL 13C-1

IULPROG
TO
655369

SOL VENT COC13

Sint

$35211.27 \mathrm{C}^{2} \mathrm{~Hz}$ $0.537281 \mathrm{~Hz}$ $0.9306754 \mathrm{sec}$ 80.6 14.200 use

$\begin{array}{rr}\text { JE } & 6.00 \text { usec } \\ \text { TE } & 297.1 \mathrm{k} \\ \mathrm{j} ! & 2.0606000 \mathrm{jec}\end{array}$

$\begin{array}{ll}\text { II } & \text { 2. } 06006000 \mathrm{sec} \\ \text { I1) } & 0.03000000 \mathrm{sec}\end{array}$

JEL TA 1 1 $89999998 \mathrm{sec}$

MCAES $\quad 0.00000000 \mathrm{sec}$

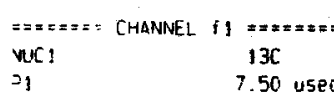

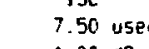

7.50 usec
$-1.00 \mathrm{OB}$
$\mathrm{O}$

Fro1 $125.7728799 \mathrm{MHZ}$

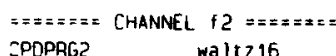

Wue?

उCPD

$x$ :

$x .12$
$x .13$

80.00 use

$-1.40 \mathrm{~dB}$

$16.66 \mathrm{~dB}$

SF02 $500.1320005 \mathrm{dH}$

=2 - Processing Darameters

SI $\quad 32768$

SF

SSB

GB

$\mathrm{EM}$
0
$100 \mathrm{H}$

$\triangle C$

1.40

10 Nur plot parameters
$C x$
$20.00 \mathrm{~cm}$

$\begin{array}{ll}\mathrm{CY} & 8.00 \mathrm{~cm} \\ \mathrm{FJP} & 200.000 \mathrm{ppm}\end{array}$

$200.000 \mathrm{ppm}$
$75151.56 \mathrm{~Hz}$

$\begin{array}{ll}=2 \mathrm{p} & 25151.56 \mathrm{~Hz} \\ =2 & -19.996 \mathrm{pDm} \\ =2 & -2514.69 \mathrm{~Hz}\end{array}$

DPMCM $\quad 10.99992$ DDOM

$\mathrm{HZCM} \quad 1383.31262 \mathrm{~Hz} / \mathrm{Cm}$ 


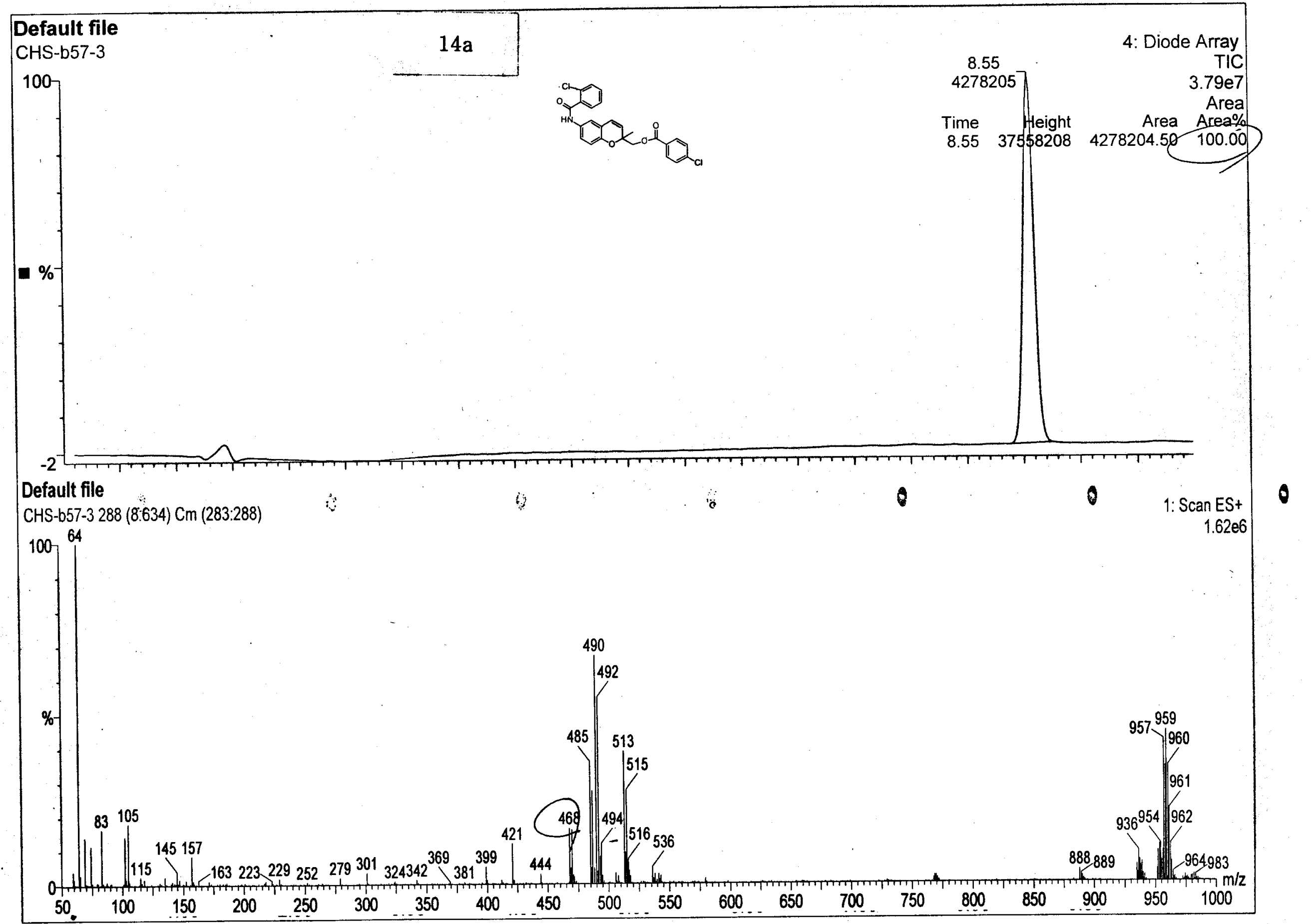



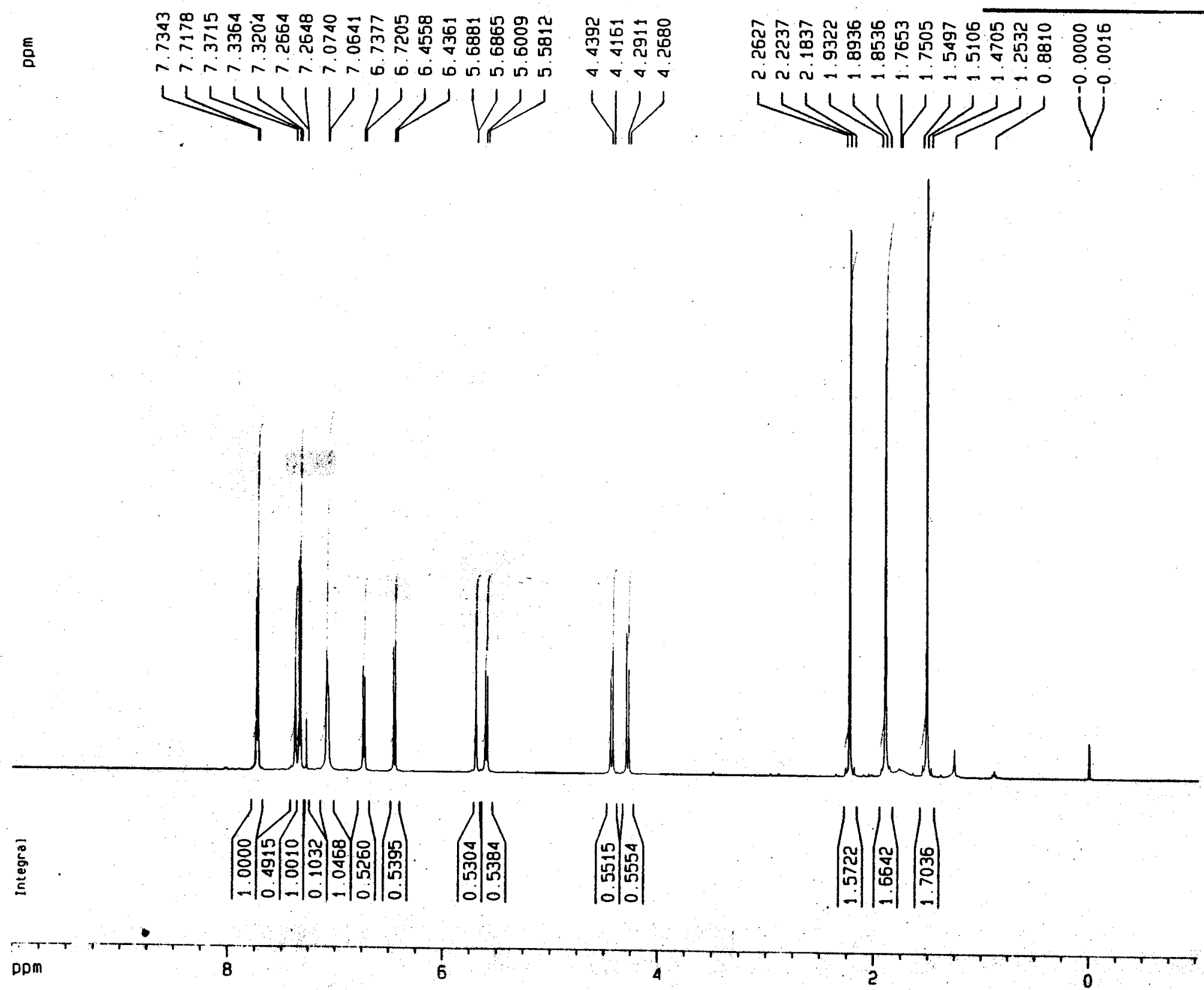

Current Dato Parameters

NAME

EXPNO

PROCNO

F2 - Acquisition Parameters

Date_ 20040923

Time

I ime

spect

$13 C-1$

TO

SOLVEN

NS

DS
SWH
FIDRES

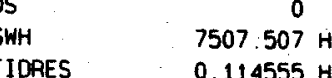

AO. $\quad 0.114555 \mathrm{~Hz}$

PG $\quad 90.5$

DW . 66.500

6.00 usec

$0.0 \mathrm{~K}$

IE $0.0 \mathrm{~K}$

D1 $\quad 1.00000000 \mathrm{sec}$

MCREST $\quad 0.00000000$ sec

MCWRK $\quad 0.01500000 \mathrm{sec}$

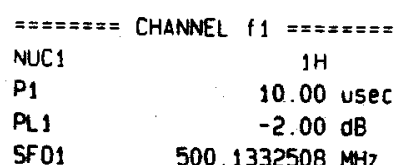

SF01 $\quad 500.1332508 \mathrm{MHz}$

F2 - Processing parameters

SI 32768

SF $\quad 500.1300107 \mathrm{MHZ}$

WOW EM

SSB $\quad 0$.

LB

GB

$0.30 \mathrm{~Hz}$
0

10 NMA plot parameters

CX $\quad 20.00 \mathrm{~cm}$

$\begin{array}{lr}\mathrm{CY} & 10.00 \mathrm{~cm} \\ \text { FIP } & 10.000 \mathrm{cPm}\end{array}$

F1 $\quad 5001.30 \mathrm{~Hz}$.

F2P $\quad-1.031 \mathrm{pom}$

F2 $\quad-515.87 \mathrm{~Hz}$

PPMCM $\quad 0.55157 \mathrm{pom} / \mathrm{cm}$

$\mathrm{HZCM} \quad 275.85828 \mathrm{~Hz} / \mathrm{cm}$ 


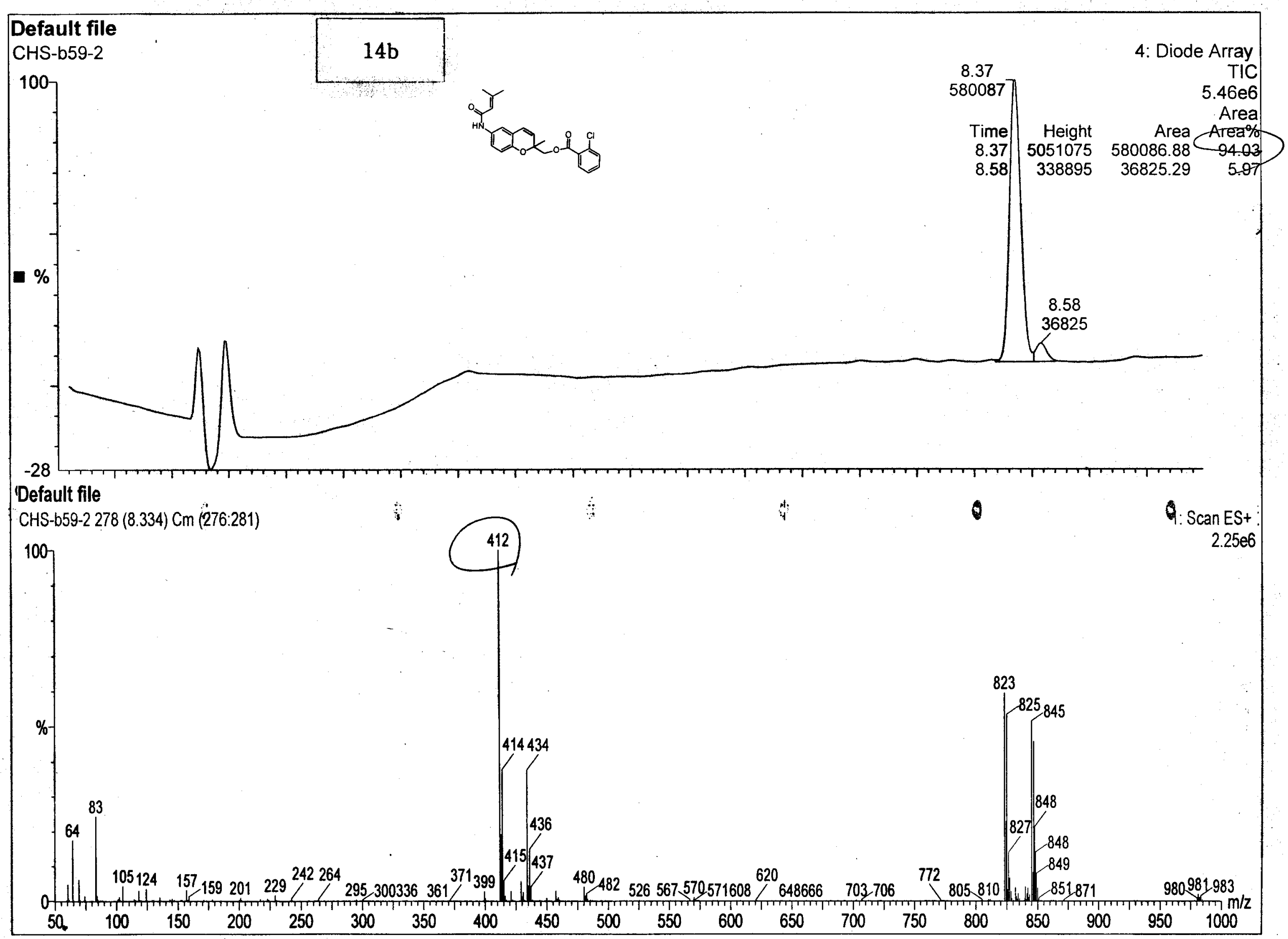




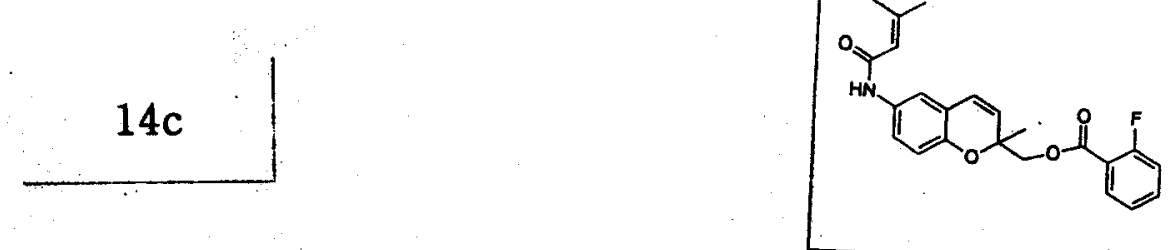

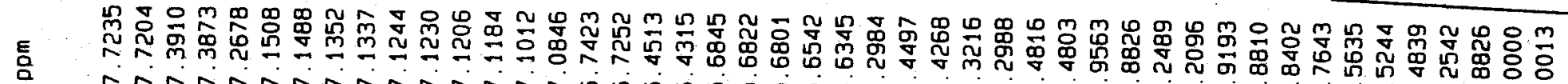

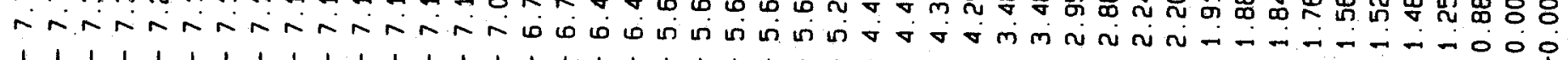
(

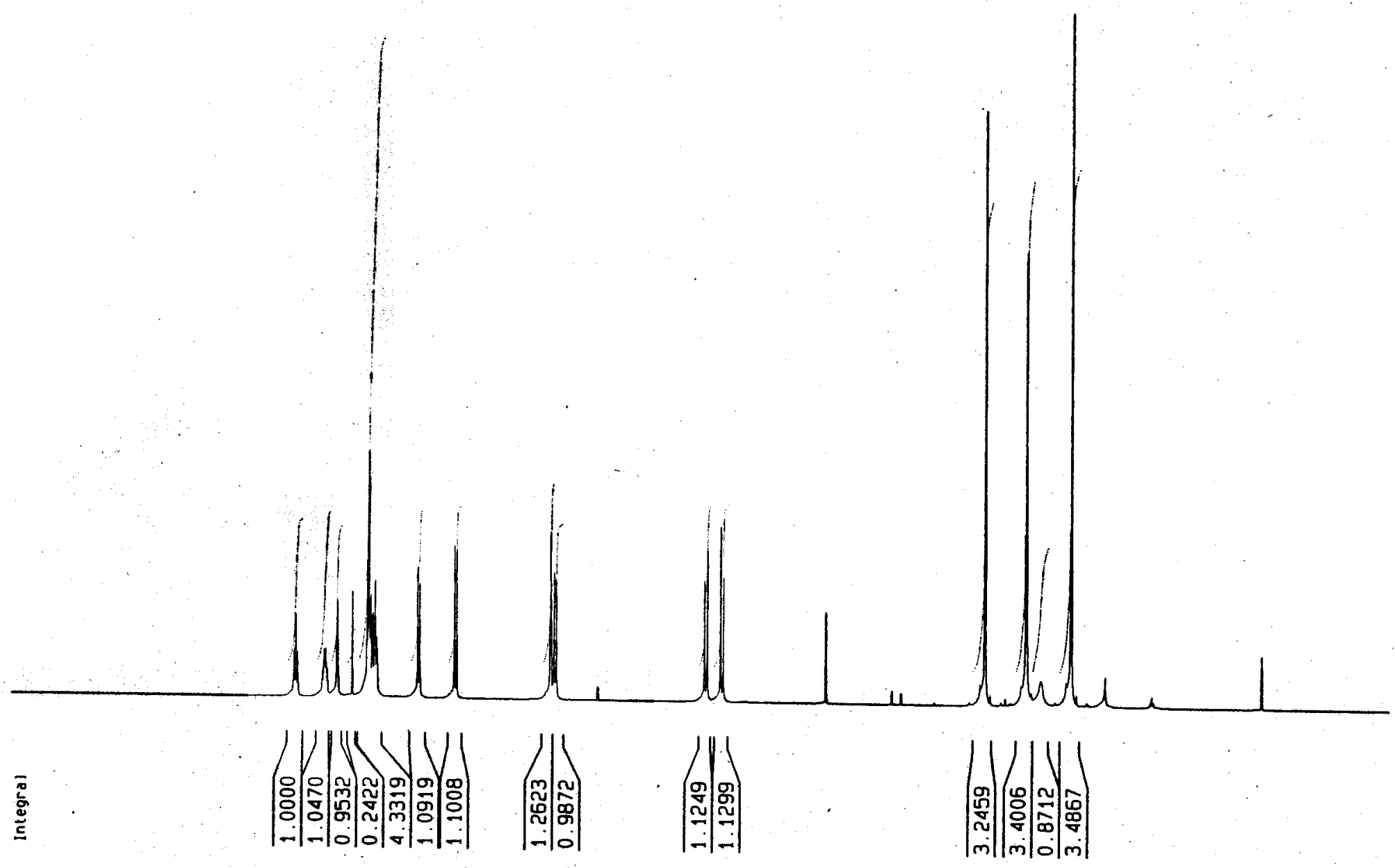

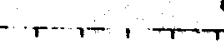

chs-b59-5 PROCNO

F2 - Acquisition Parameters

Date_ 20040923

Time $\quad 6.17$

spect

PROBHD $5 \mathrm{~mm}$ DUL $13 \mathrm{C}-1$

PULPROG

TD

SOLVENT

NS
OS
SWH

SWH

FID

AQ

RG

DW

DE
TE
D1

MCREST

29
65536

$\operatorname{CDC} 13$

MCURK

NUC 1

$P_{1}$

PL1

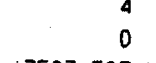

$7507.507 \mathrm{~Hz}$

$0.114555 \mathrm{~Hz}$

4. 3648143 sec 90.5

66.600 usec

6.00 usec

$0.00000000 \mathrm{sec}$

$0.01500000 \mathrm{sec}$

SFO1 $500.1332508 \mathrm{MHZ}$

F2 - Processing parameters

SI 32768

SF $\quad 500.1300096 \mathrm{MHz}$

WDW

SSB

LB

PC

EM
0
$0.30 \mathrm{~Hz}$
0
1.00

10 NMP plot parameters

CX $20.00 \mathrm{~cm}$

$\begin{array}{ll}\mathrm{cY} & 20.00 \mathrm{~cm} \\ \mathrm{Fy} & 10.00 \mathrm{~cm}\end{array}$

F1P $\quad 10.000 \mathrm{ppm}$

F1 $\quad 5001.30 \mathrm{~Hz}$

F2P - -1.031 DOD

$2 \quad-515.87 \mathrm{~Hz}$

PPMCM $\quad 0.55157 \mathrm{pom} / \mathrm{cm}$

HZCM $275.85828 \mathrm{~Hz} / \mathrm{cm}$ 


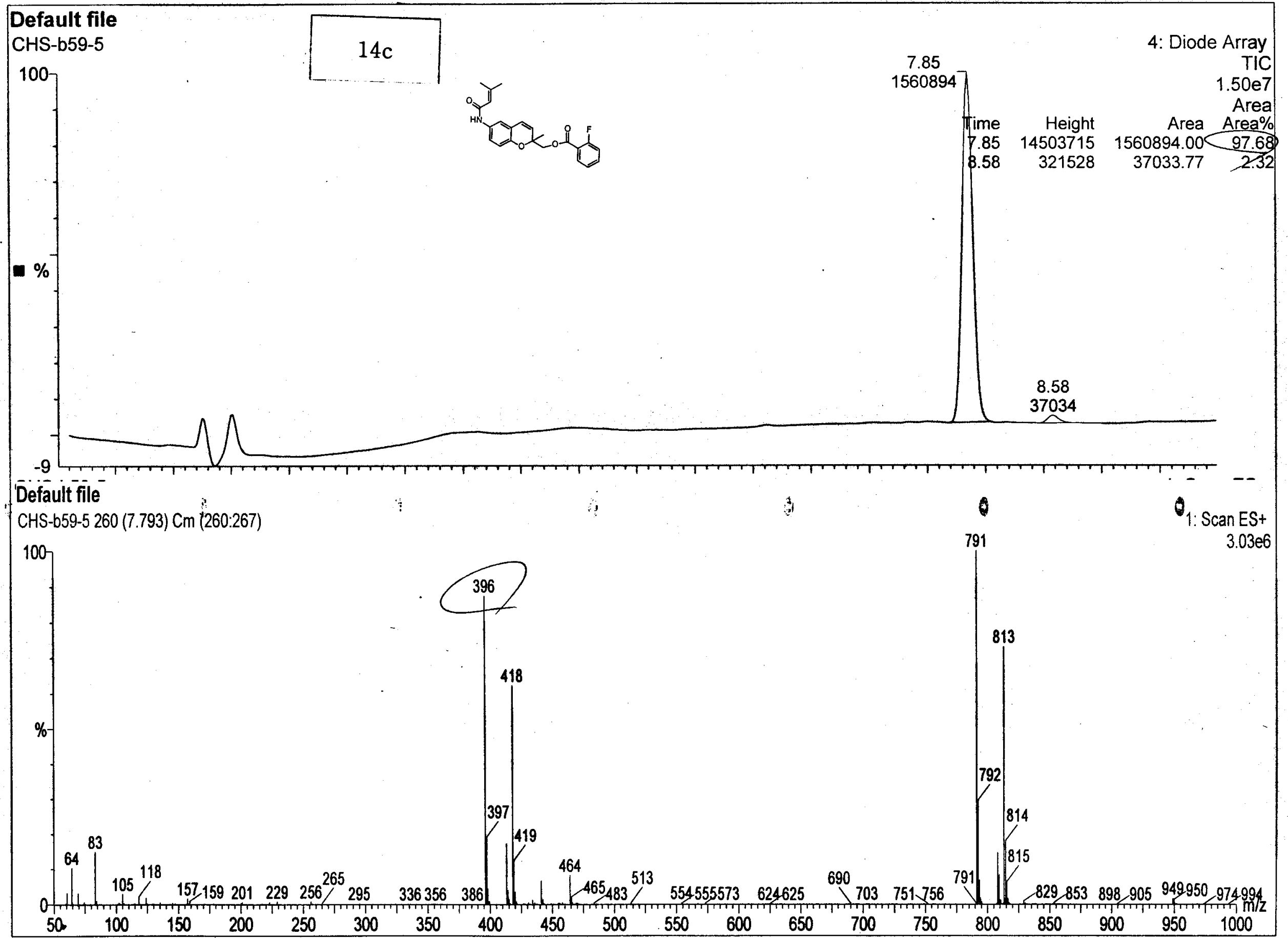




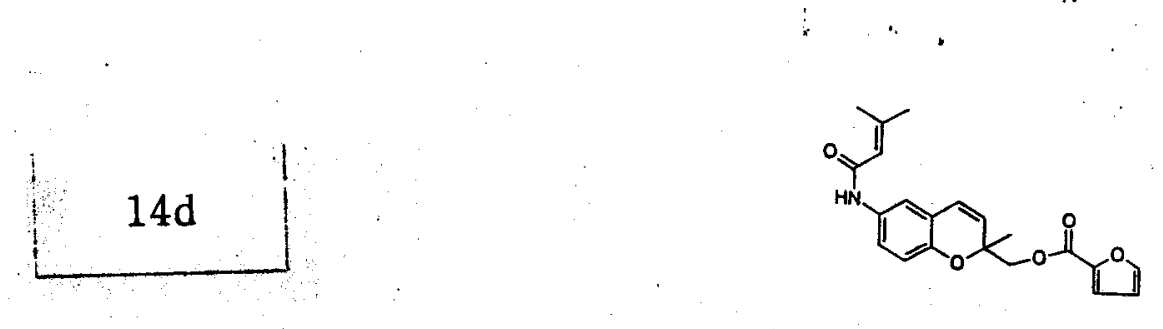

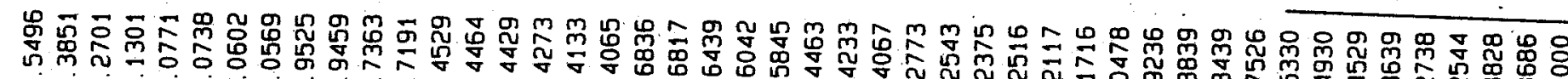

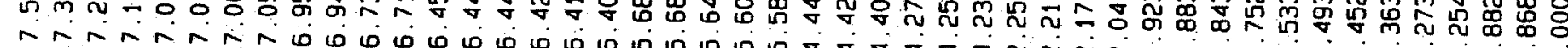

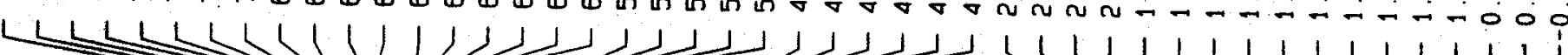
กา1
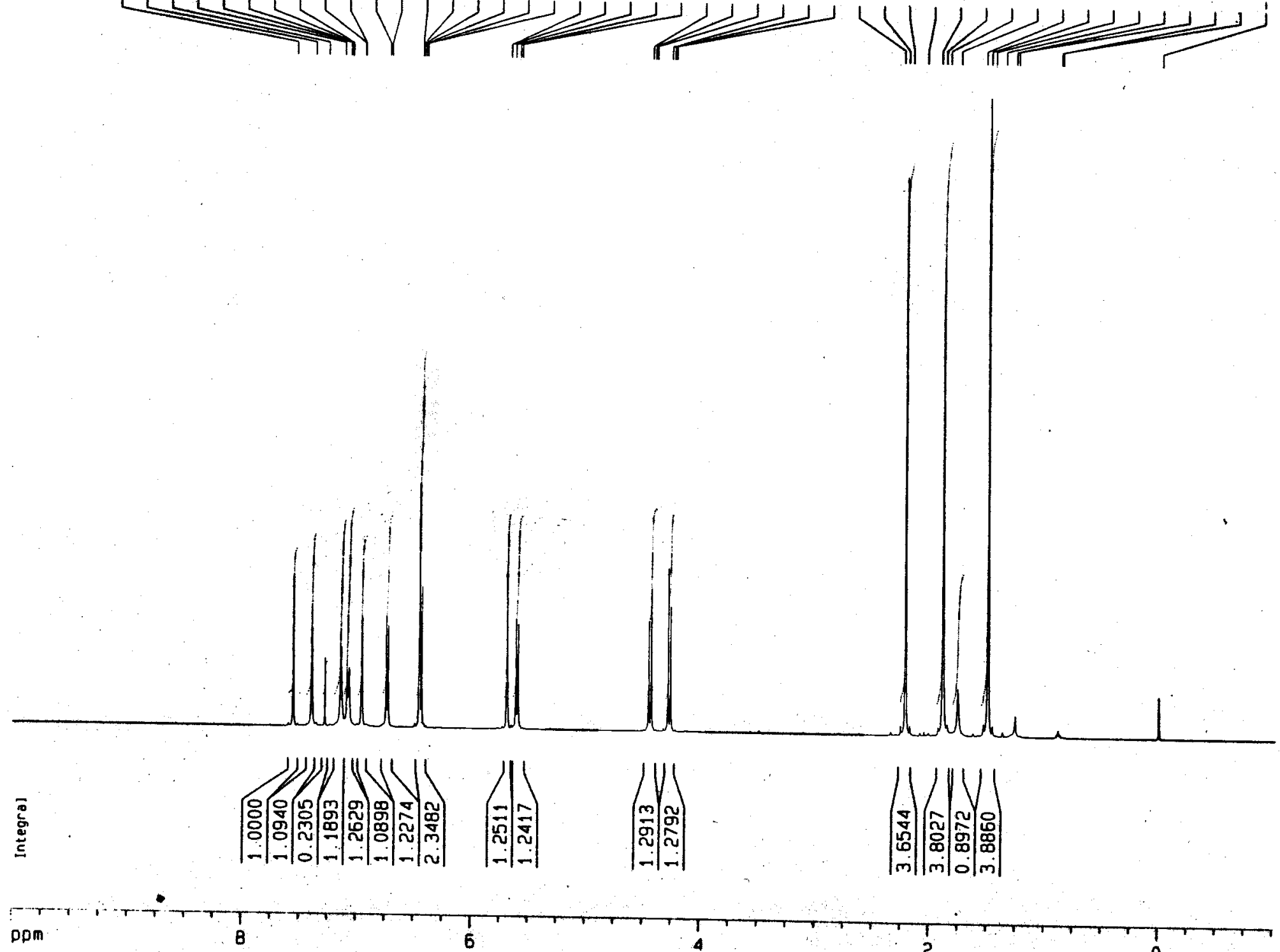

Current Data Parameters NAME chs-b59-7 PROCNO

F2 - Acquisition Parameters

Date

Time 20040923 6.27

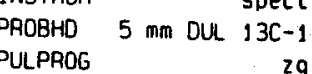
PULPROG TO

SOLVENT

NS

FIORES

$A Q$

RG

DW

DE

Di

MCRES

MCWRK

65536

$\operatorname{coc} 13$ 4

$7507.507 \mathrm{~Hz}$

$0.114555 \mathrm{~Hz}$

4. $3648143 \mathrm{sec}$

71.8

66.500 usec

6.00 use

$0.0 \mathrm{~K}$

$1.00000000 \mathrm{sec}$

$0.00000000 \mathrm{sec}$

$==z==s=$ CHANNEL f $1==x \pi z=0$

NUC

P1

10.00 use

SF01 500.1332508 OB

2 - Processing parameters

SI 32768

SF $500.1300084 \mathrm{MHz}$

WOW EM

$E M$
0
$0.30 \mathrm{~Hz}$
0

LB

$P C$

1.00

10 NMR plot parameters

CX $\quad 20.00 \mathrm{~cm}$

CY $\quad 10.00 \mathrm{~cm}$

F1P $\quad 10.000 \mathrm{pDm}$

$\mathrm{FI}^{2} \quad 5001.30 \mathrm{~Hz}$

F2P $\quad-1.031 \mathrm{Dpm}$

F2 $\quad-515.87 \mathrm{~Hz}$

$\begin{array}{lr}\text { PPMCM } & 0.55157 \mathrm{pPm} / \mathrm{Cm} \\ \mathrm{HZCM} & 275.85828 \mathrm{~Hz} / \mathrm{cm}\end{array}$ 


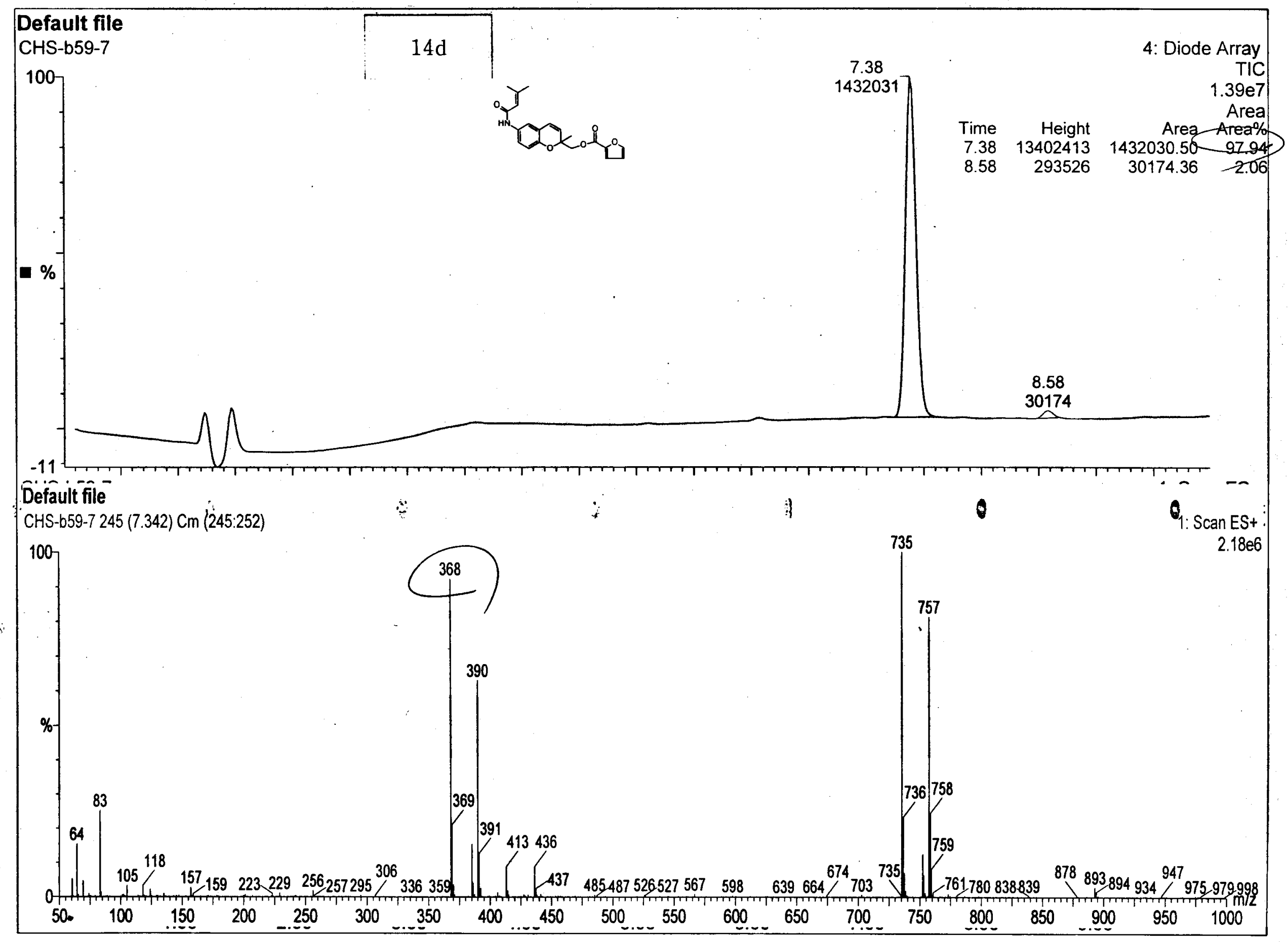




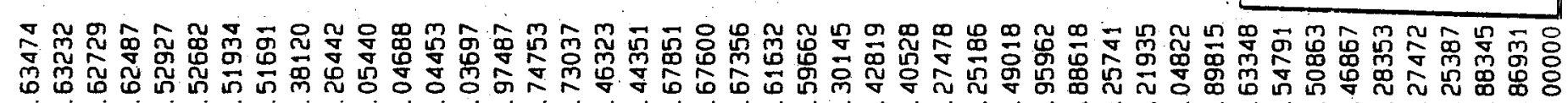
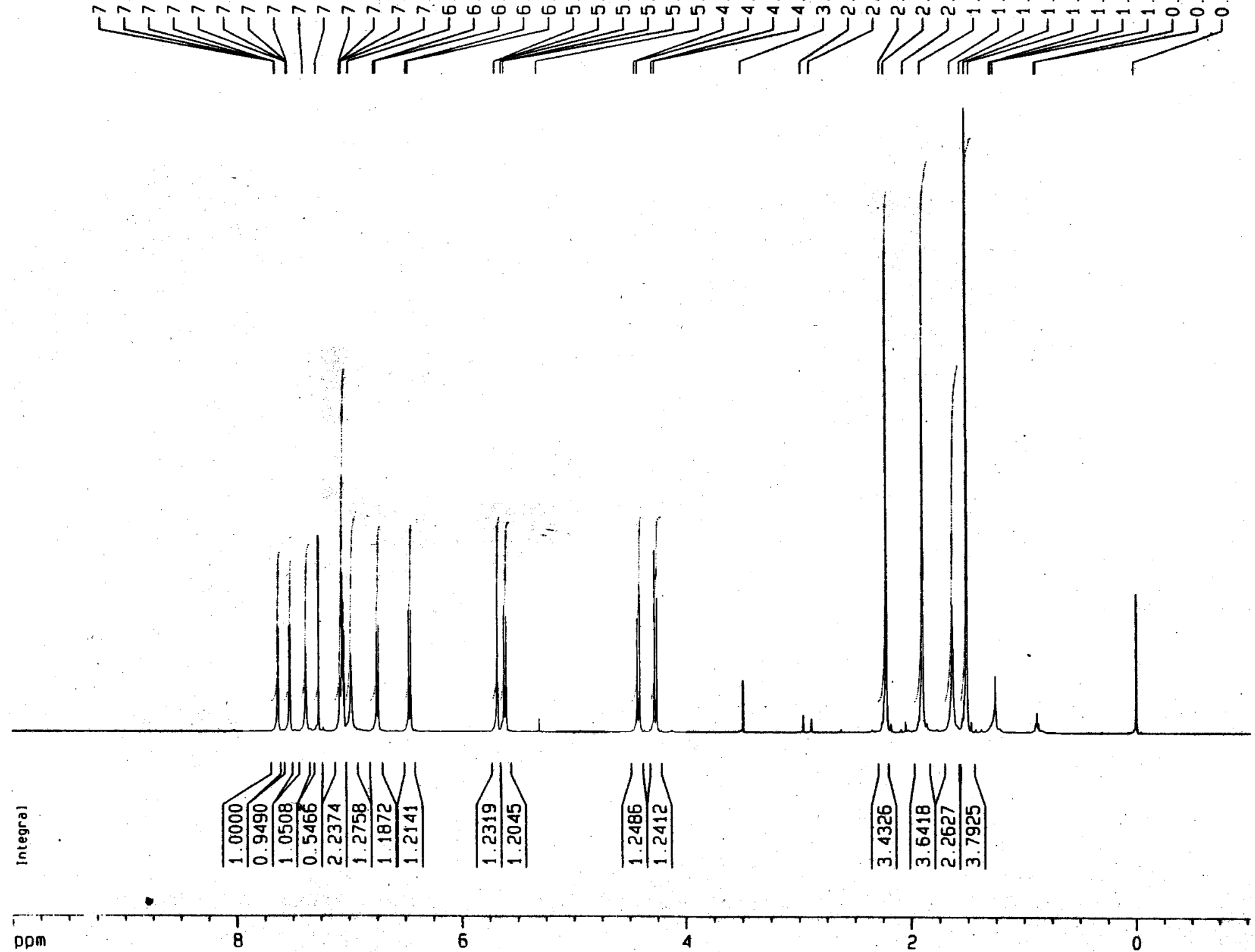

Current Data Parameters chs-b59-9

EXPNO

PROCNO

F2 - Acquisition Parameters

Date

20040923

Time $\quad 6.37$

INSTRUM Spect

PROBHD 5 mIM DUL $13 \mathrm{C}-1$

PULPROG

TD

SOLVEN

NS

DS

FIDRES

$\Delta 0$

AO

RW

DE

TE

01

MCAEST

29
65536

$\mathrm{COC}] 3$

0
0
0.114507

$0.114555 \mathrm{~Hz}$

4.3648143 sec

143.7 66.600 usec 66.600 usec
6.00 usec

$0.0 \mathrm{~K}$
$1.00000000 \mathrm{~K}$

$1.00000000 \mathrm{sec}$

0.00000000 sec

$\quad 0.01500000 \mathrm{sec}$

$== \pm= \pm=$ CHANNEL ff $== \pm== \pm$ NUC1

P1 $10^{14}$

SF01 $\quad 500.1332508 \mathrm{MHz}$

F2 - Processing parameters

SI - Processing Darameters

SF $\quad 500.1300110$ MH-1

WOW

LB $\quad 0.30 \mathrm{~Hz}$

$\begin{array}{lr}\text { GB } & 0 \\ \text { PC } & 1.00\end{array}$

10 NMR plot parameters

CX $20.00 \mathrm{~cm}$

Cr $\quad 10.00 \mathrm{~cm}$

F1P $\quad 10.000 \mathrm{pDm}$

F1 $5001.30 \mathrm{~Hz}$

F2P -1.031 DDm

F2 $\quad-515.87 \mathrm{HZ}$

$\begin{array}{lr}\text { PPMCM } & 0.55157 \mathrm{pDm} / \mathrm{Cm} \\ \mathrm{HZCM} & 275.85828 \mathrm{~Hz} / \mathrm{Cm}\end{array}$ 


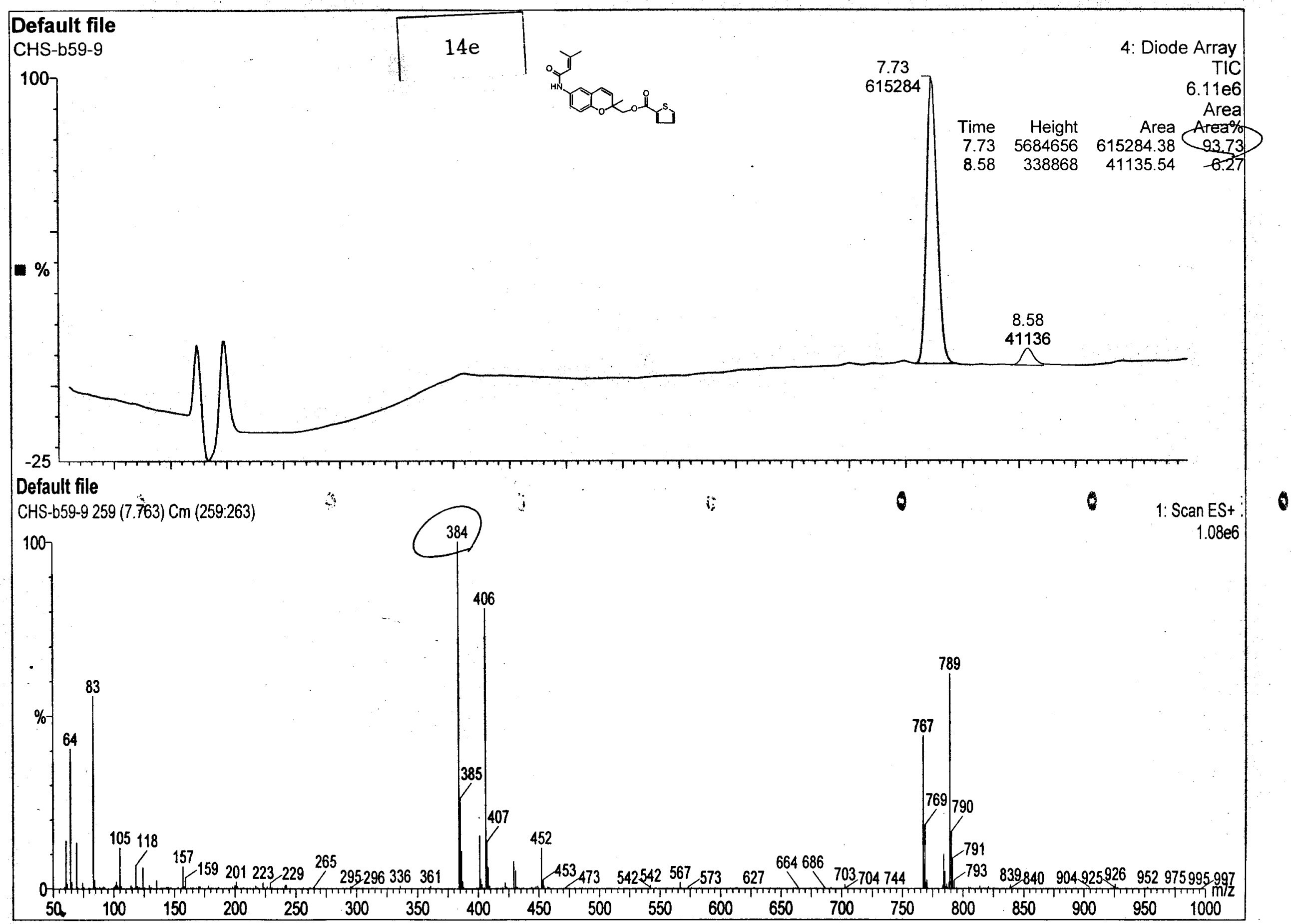




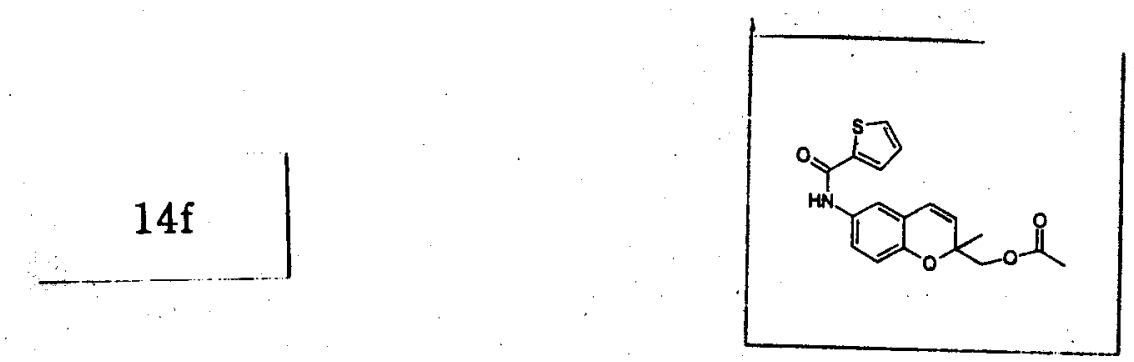

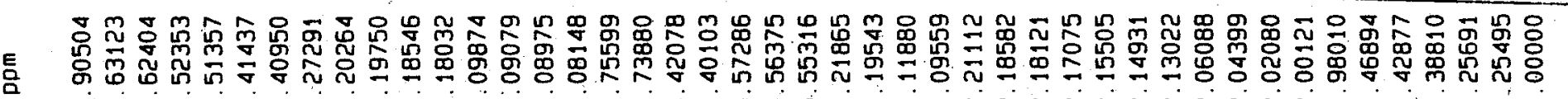

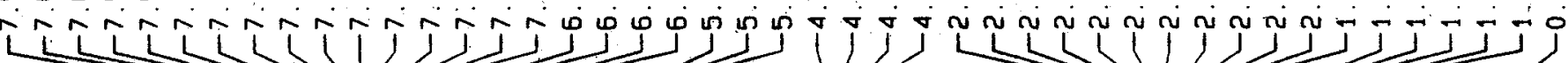

$\ldots 1$
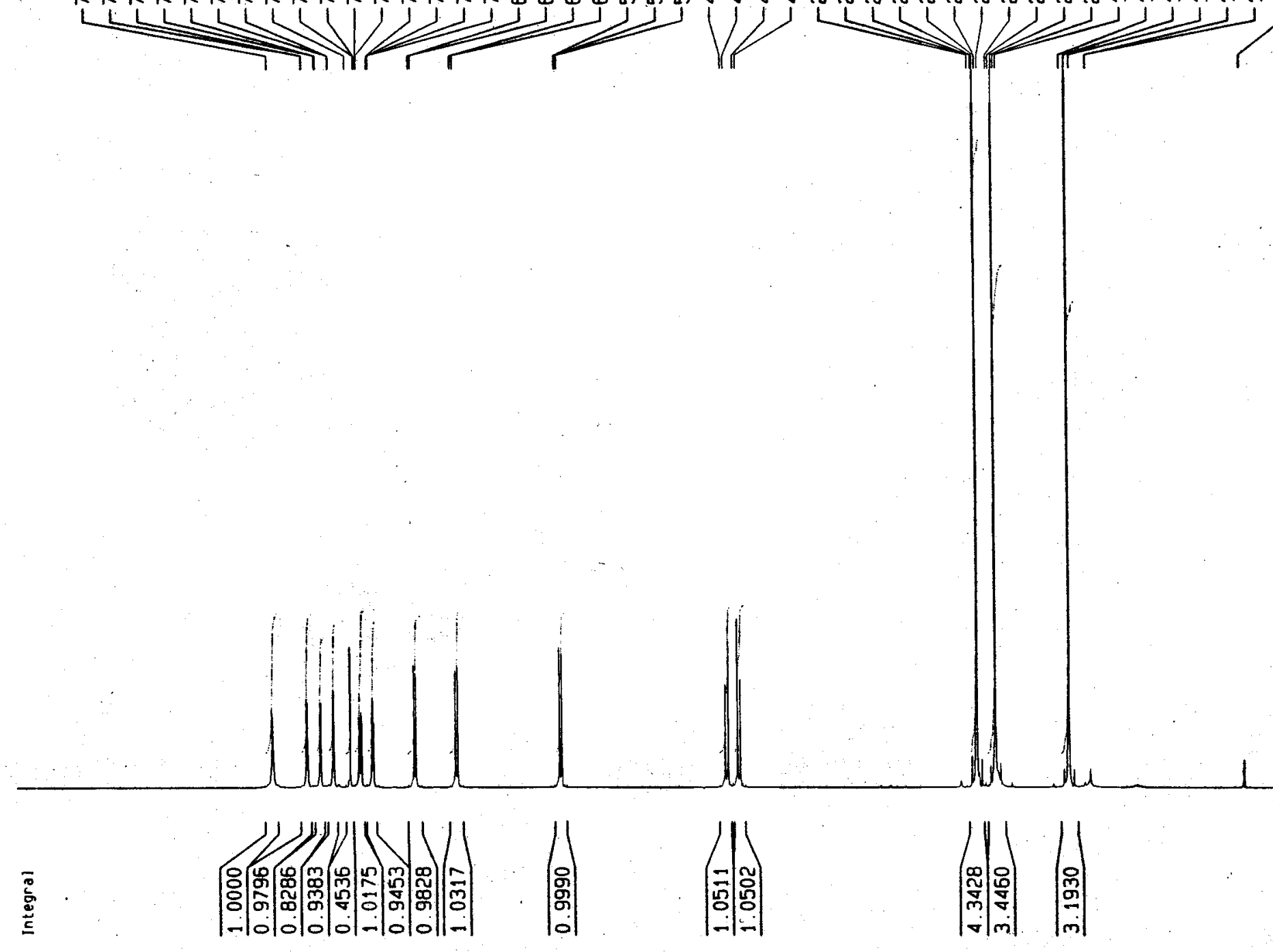

\begin{tabular}{l} 
Current Data Parameters \\
NAME \\
EXPNO \\
PROCNO \\
PRO-b71-1 \\
\hline
\end{tabular}

F2 - ACquisition Parameters 20041013

Time

17.35

INSTRUM Spect

PROBHD $5 \mathrm{~mm}$ DUL $13 \mathrm{C}-1$

PULPAOG

PULPAO
ID
SOLVEN

NS

DS

SWH
FIDRES

FIDRES
AQ
RG
DH

DW

DE

TE

MCRES

65536

$\operatorname{COC} 13$

0

$0.114555 \mathrm{~Hz}$

$0.114555 \mathrm{~Hz}$

4. $3648143 \mathrm{sec}$

66.600 usec

6.00 usec

$0.0 \mathrm{~K}$

$1.00000000 \mathrm{sec}$

$0.00000000 \mathrm{sec}$

MCWAK $\quad 0.01500000 \mathrm{sec}$

$==z=z=$ CHANNEL $f 1=x=m=x=$

$\begin{array}{ll}\text { NUC1 } & 1 \mathrm{H} \\ \mathrm{P}_{1} & 10.00 \mathrm{usec}\end{array}$

$\begin{array}{ll}P_{1} & 10.00 \text { use } \\ P_{L 1} & -2.00 \mathrm{~dB}\end{array}$

SFO1 $500.1332508 \mathrm{MHz}$
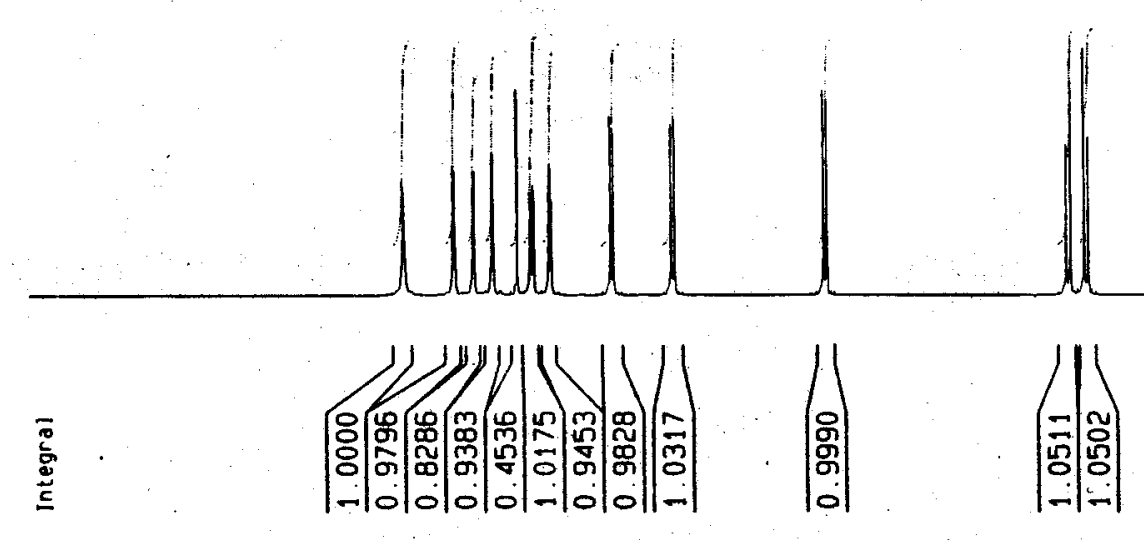

F2 - Processing parameters

S1 32768

SF $\quad 500.1300056 \mathrm{MHZ}$

WOW $\quad$ EM

LB $\quad 0.30 \mathrm{~Hz}$

PC $\quad 0$

10 NMA plot parameters

CX $20.00 \mathrm{~cm}$

$\begin{array}{ll}\text { CX } & 20.00 \mathrm{~cm} \\ \mathrm{CY} & 20.00 \mathrm{~cm}\end{array}$

$\begin{array}{lr}\text { CY } & 20.00 \mathrm{~cm} \\ \text { F1P } & 10.000 \mathrm{ppm}\end{array}$

F1 $\quad 5001.30 \mathrm{~Hz}$

$\begin{array}{ll}F 2 P & -1.030 \mathrm{DPm} \\ \mathrm{F2} & -515.20 \mathrm{~Hz}\end{array}$

PPMCM $\quad 0.55151 \mathrm{ppm} / \mathrm{Cm}$

HZCM $275.82523 \mathrm{~Hz} / \mathrm{cm}$ 


\section{$14 \mathrm{f}$}

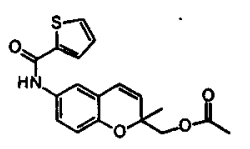

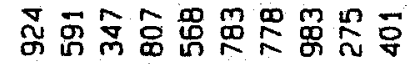

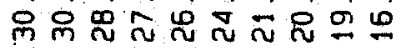

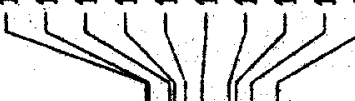

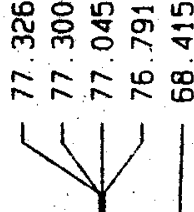

要品

กำ

1
Uurrent Data Parameters

NAME

DROCNO

$=2-$ Acquisstion

Tate. Acqussition Parameters

$\begin{array}{lr}\text { Time } & 20050714 \\ & 16.18\end{array}$

INSTRUM spect

an $13 \mathrm{C}-1$

Tit

SOLVNT COC 13
CSET

$\begin{array}{rr}\text { VS } & 1024 \\ \text { JS } & 2\end{array}$

5 SWH $\quad 35211.270 \mathrm{~Hz}$

$0.9306754 \mathrm{sec}$

$26 \quad 101.6$

$3 \mathrm{~W}$
$\mathrm{JE}$

6.00 usec

$2.000000005 \mathrm{se}$

.03000000 sec

. 89999998 ser

HCHFK $\quad 0.01500000 \mathrm{sec}$

$= \pm=x=z=$ CHANNEL $f 1=z=z==$

$\begin{array}{ll}\text { WC1 } & 13 C \\ { }_{21} & 7.50 \text { use }\end{array}$

ग) $-100 \mathrm{~dB}$

SFOI $\quad 125.7728799 \mathrm{MHZ}$

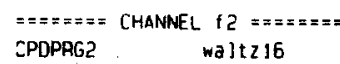

CPDPRG2 wajtz 16
vUC2

गCPO2 80.00 usec

$\begin{array}{ll}\text { HL2 } & -1.40 \mathrm{OB} \\ \text { HL12 } & 16.66 \mathrm{OB}\end{array}$

x.13 $25.00 \mathrm{OB}$

$5 F 02 \quad 500.1320005 \mathrm{MHz}$

F2 - Processing parameters
S1 32768

$\begin{array}{lr}\text { S1 } & 32768 \\ \text { SF } & 125.7577890 \mathrm{MHZ}\end{array}$

NOW $\quad$ EM

$\begin{array}{ll}2 B & 1.00 \mathrm{~Hz}\end{array}$

1.40

10 Nur plot parameters

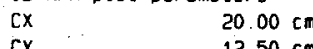

Fip $\quad 12.50 \mathrm{~cm}$

F1 25151.56 Hz

$\begin{array}{ll}\text { F2P } & 25151.56 \mathrm{Kz} \\ \text { F } & -19.996 \mathrm{DDm}\end{array}$

F2 $\quad-2514.69 \mathrm{~Hz}$

$\begin{array}{lr}\text { PPMCM } & 10.99982 \mathrm{ppm} / \mathrm{cm} \\ \mathrm{HZCM} & 1383.31262 \mathrm{~Hz} / \mathrm{cm}\end{array}$ 


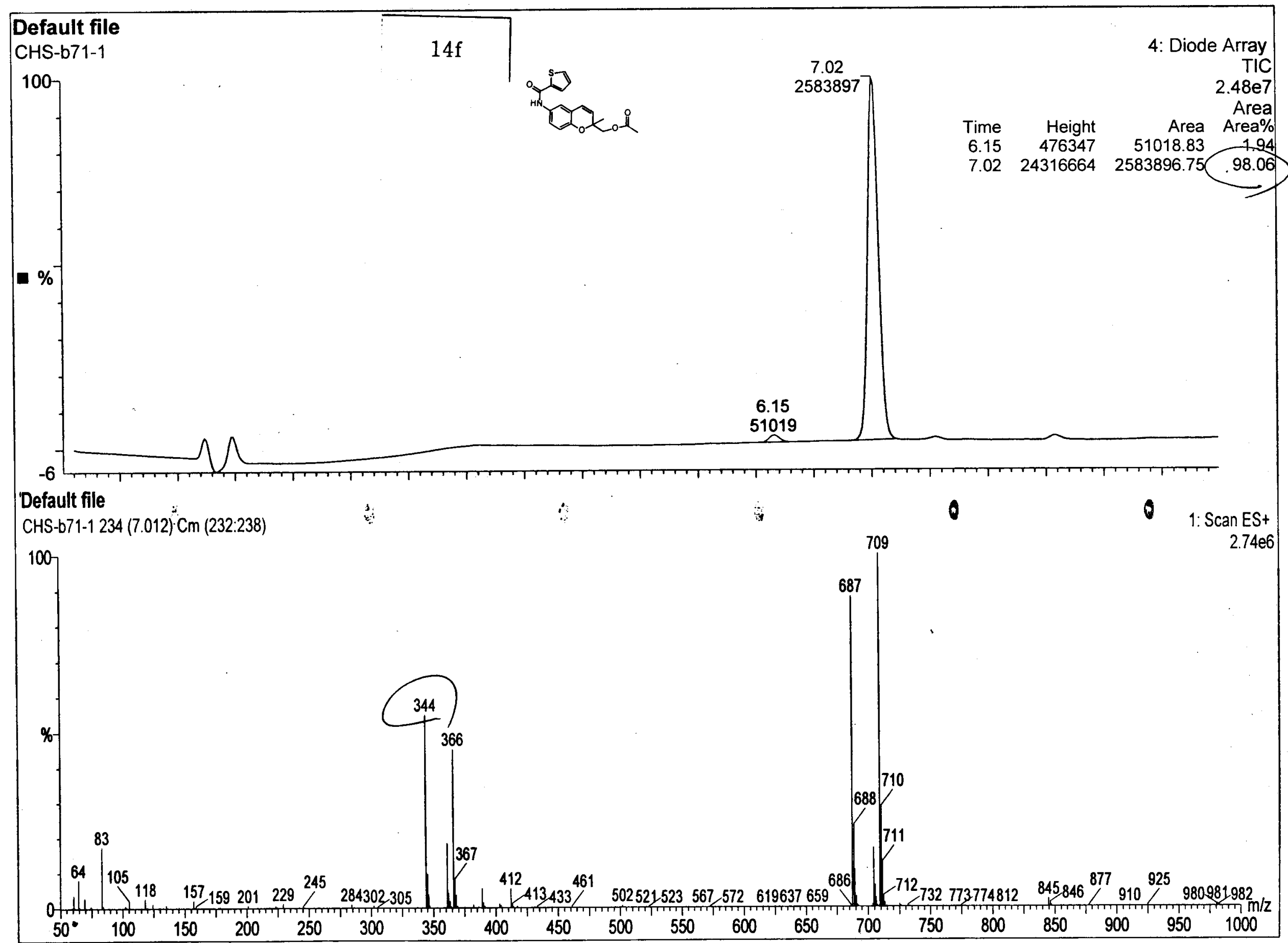




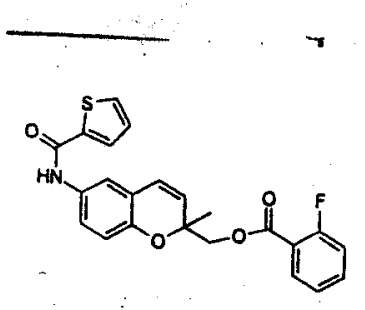

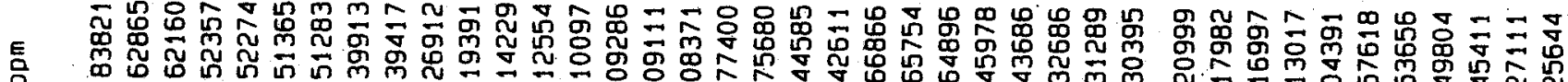

$14 \mathrm{~g}$

íni

号.

NAME

Current Data Parameters

NAME

PAOCNO
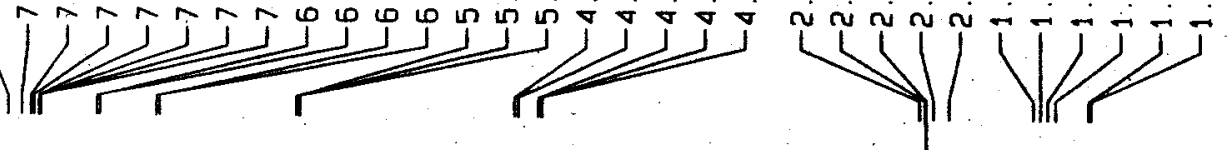

F2 - Acquisition Parameters

Oate_ 20041013

Time $\quad 17.54$

INSTRUM Spect

PROBHD $5 \mathrm{~mm}$ DUL $13 \mathrm{C}-1$

PULPROG 20

\begin{tabular}{lr} 
PULPROG & 29 \\
ID & 65536 \\
\hline
\end{tabular}

SOLVENT COC13

NS

DS

SWH $\quad 7507.507 \mathrm{~Hz}$

FIDRES $\quad 0.114555 \mathrm{~Hz}$

AO 4. 3648143 $\mathrm{sec}$

RG 57

OW

DE

IE

66.600 usec

6.00 usec

$0.0 \mathrm{~K}$

D1 $\quad 1.00000000 \mathrm{sec}$

MCREST $\quad 0.00000000 \mathrm{sec}$

MCWRK

$0.01500000 \mathrm{sec}$

$=z==s==$ CHANNEL $f 1==s===$
NUC1

$\begin{array}{ll}\text { NUC1 } & 1 H \\ P_{1} & 10.00 \text { use }\end{array}$

$\begin{array}{ll}P_{1} & 10.00 \text { use } \\ D_{L 1} & -2.00 \mathrm{~dB}\end{array}$

SFO1 $500.1332508 \mathrm{MHZ}$

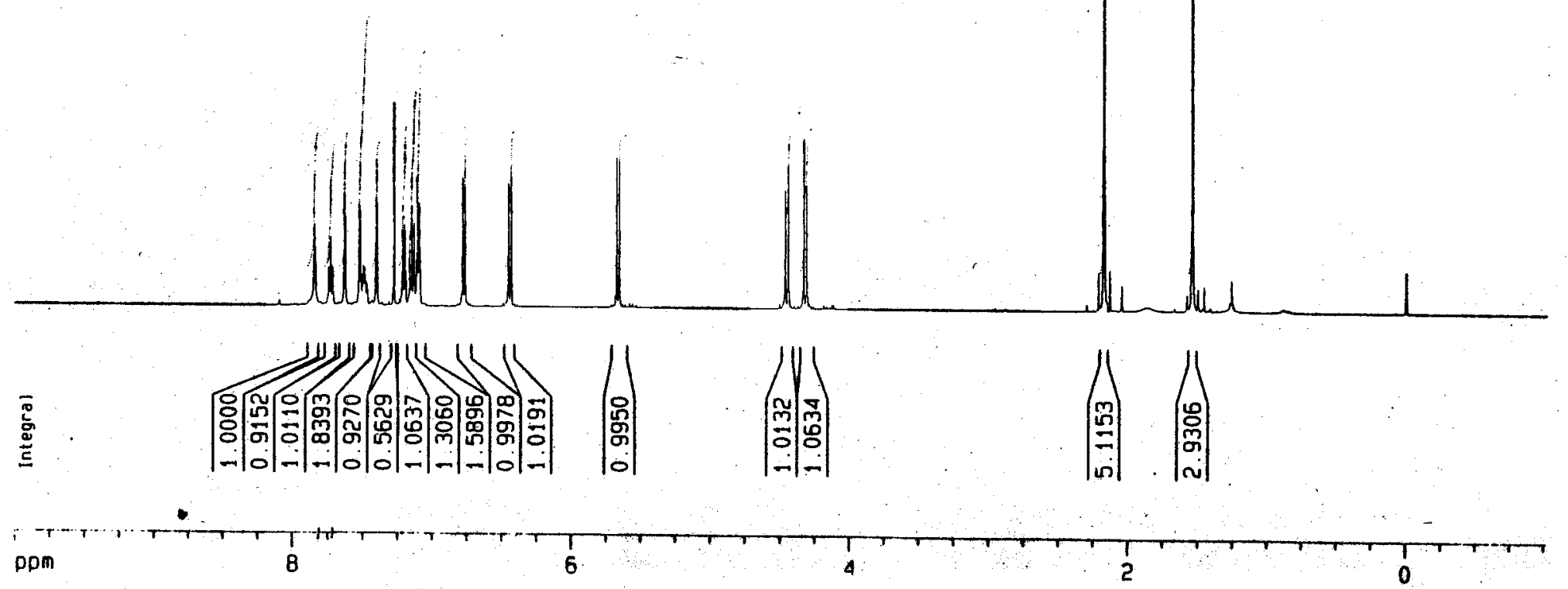

E2 - Processing parameters

SI 32768

SF $\quad 500.1300086 \mathrm{MHZ}$

WOW EM

SSB $\quad \begin{gathered}E M \\ \text { WOW }\end{gathered}$

$\begin{array}{cc}\text { LB } & 0.30 \mathrm{H} \\ \text { GB } & 0\end{array}$

PC

10 NMR plot parameters

CX $\quad 20.00 \mathrm{~cm}$

$\mathrm{Cr} \quad 25.00 \mathrm{~cm}$

FIP $\quad 10.000 \mathrm{ppm}$

F1 $\quad 5001.30 \mathrm{~Hz}$

$\begin{array}{ll}F 2 P & -1.030 \mathrm{pDm} \\ F 2 & -515.20 \mathrm{~Hz}\end{array}$

PPMCM : $0.55151 \mathrm{ppm} / \mathrm{Cm}$

HZCM $275.82523 \mathrm{~Hz} / \mathrm{cm}$ 


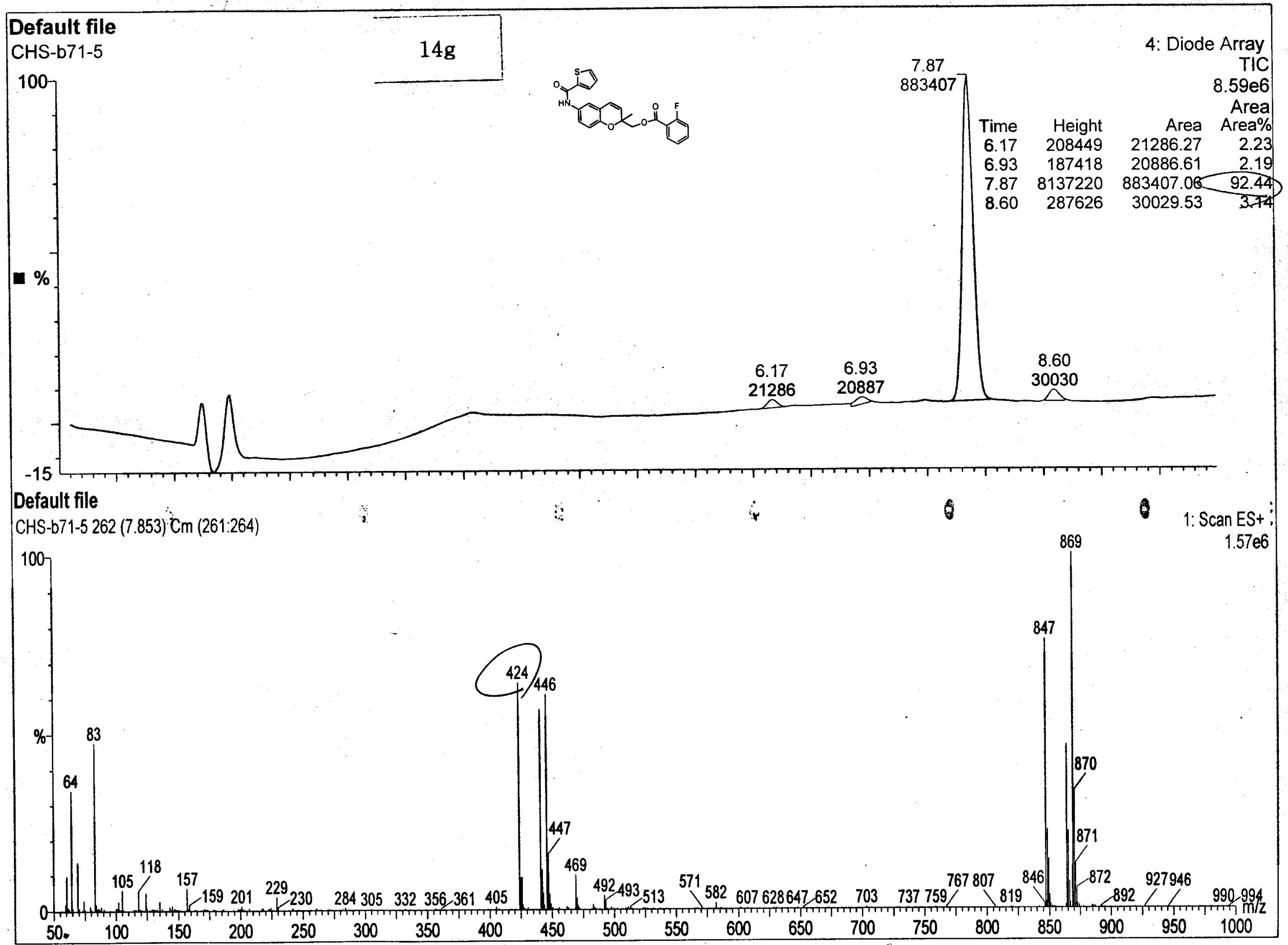




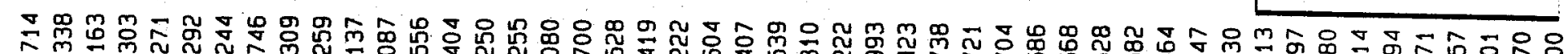

言. LLLli hl
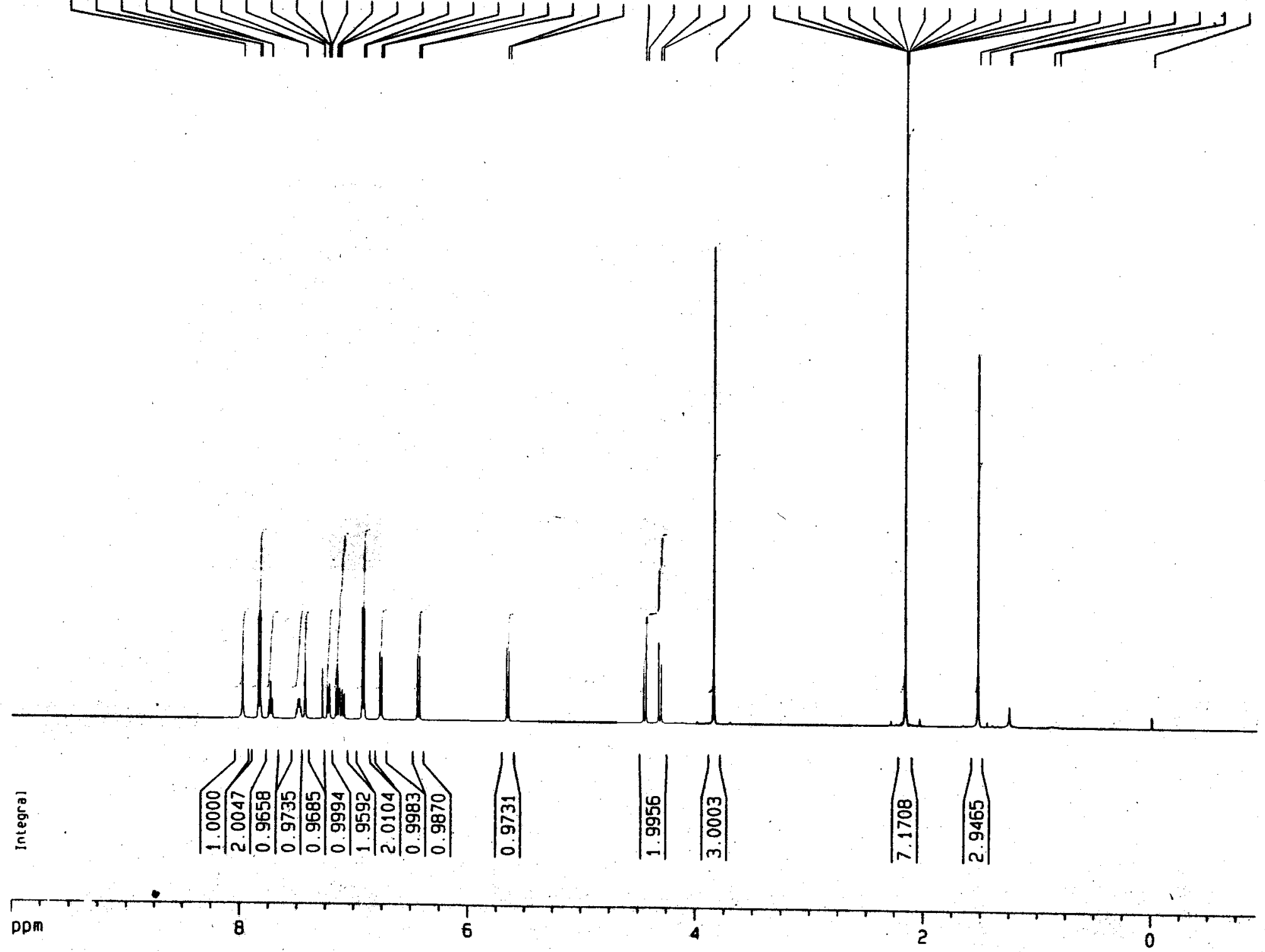

Current Data Parameters

F2 - Acquisition Parameters
Date_ 20041027

Date

2.17

5Dect

$-1$

TO
SOLVENT
NS

NS

DS

FIDRES $\quad 0775.068 \mathrm{~Hz}$

$\begin{array}{ll}\text { FIDRES } & 0.413517 \mathrm{~Hz} \\ A Q & 1.2092630 \mathrm{se}\end{array}$

RG $\quad \begin{array}{r}1.2092630 \\ 71.8\end{array}$

73.800 usec

$\begin{array}{cc}\text { DE } & 6.00 \mathrm{usec} \\ \text { TE } & 0.0 \mathrm{~K}\end{array}$

D1 $2.00000000 \mathrm{sec}$

MCREST $\quad 0.00000000 \mathrm{sec}$

MCWRK $\quad 0.01500000 \mathrm{sec}$

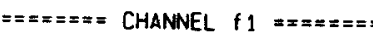

$\begin{array}{ll}\text { NUC1 } & 1 \mathrm{H} \\ P_{1} & 10.00 \text { use }\end{array}$

$\begin{array}{ll}P_{1} & 10.00 \text { use } \\ \text { PL1 } & -2.00 \mathrm{~dB}\end{array}$

SF01 $500.1329272 \mathrm{MH2}$

F2 - Processing parameters

SI 32768

SF $\quad 500.1300066 \mathrm{MHZ}$

WOW EM

SSB

68

$E M$
0

$0.30 \mathrm{~Hz}$

1.00

ID NMR plot parameters

CX Drol paraneters

$\mathrm{CY} \quad 20.00 \mathrm{~cm}$

FIP $10.000 \mathrm{CD}$

F1 $\quad 5001.30 \mathrm{~Hz}$

$F_{2 P}-0.942 \mathrm{ppm}$

F2 $\quad-470.95 \mathrm{~Hz}$

P.PMCM $0.54708 \mathrm{DDN} / \mathrm{Cm}$

HZCM $273.61273 \mathrm{~Hz} / \mathrm{Cm}$ 


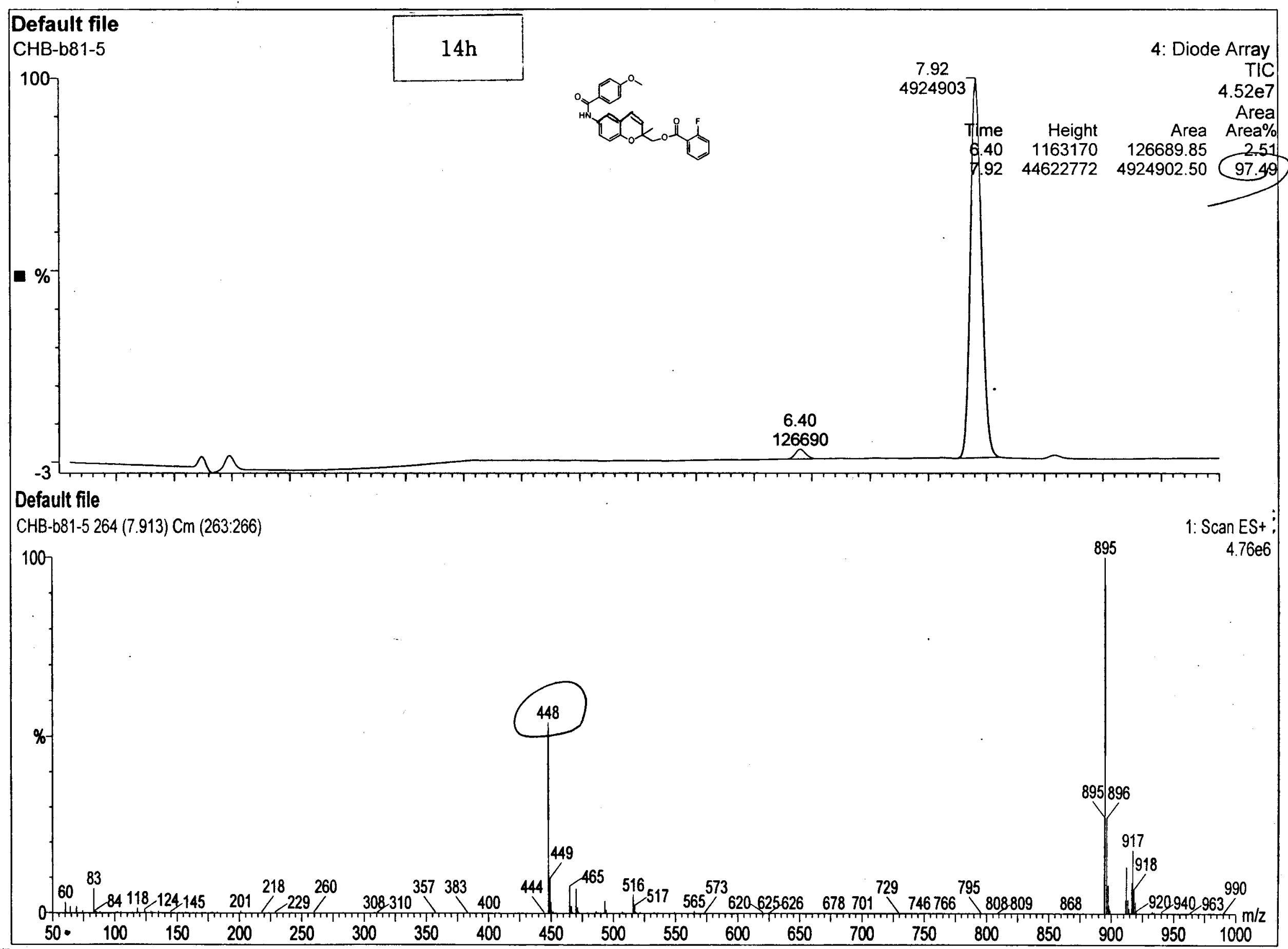




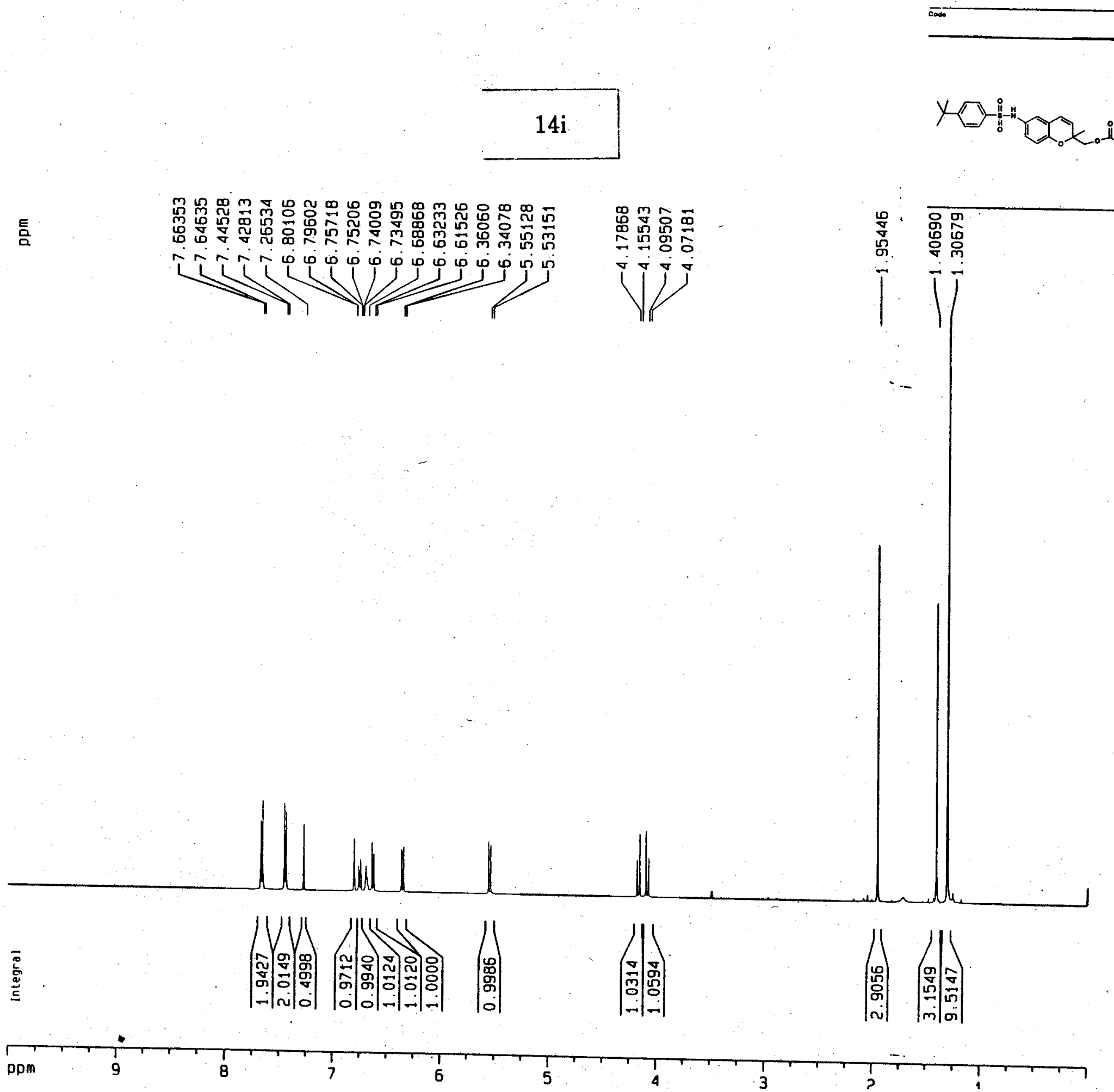

\section{S138}




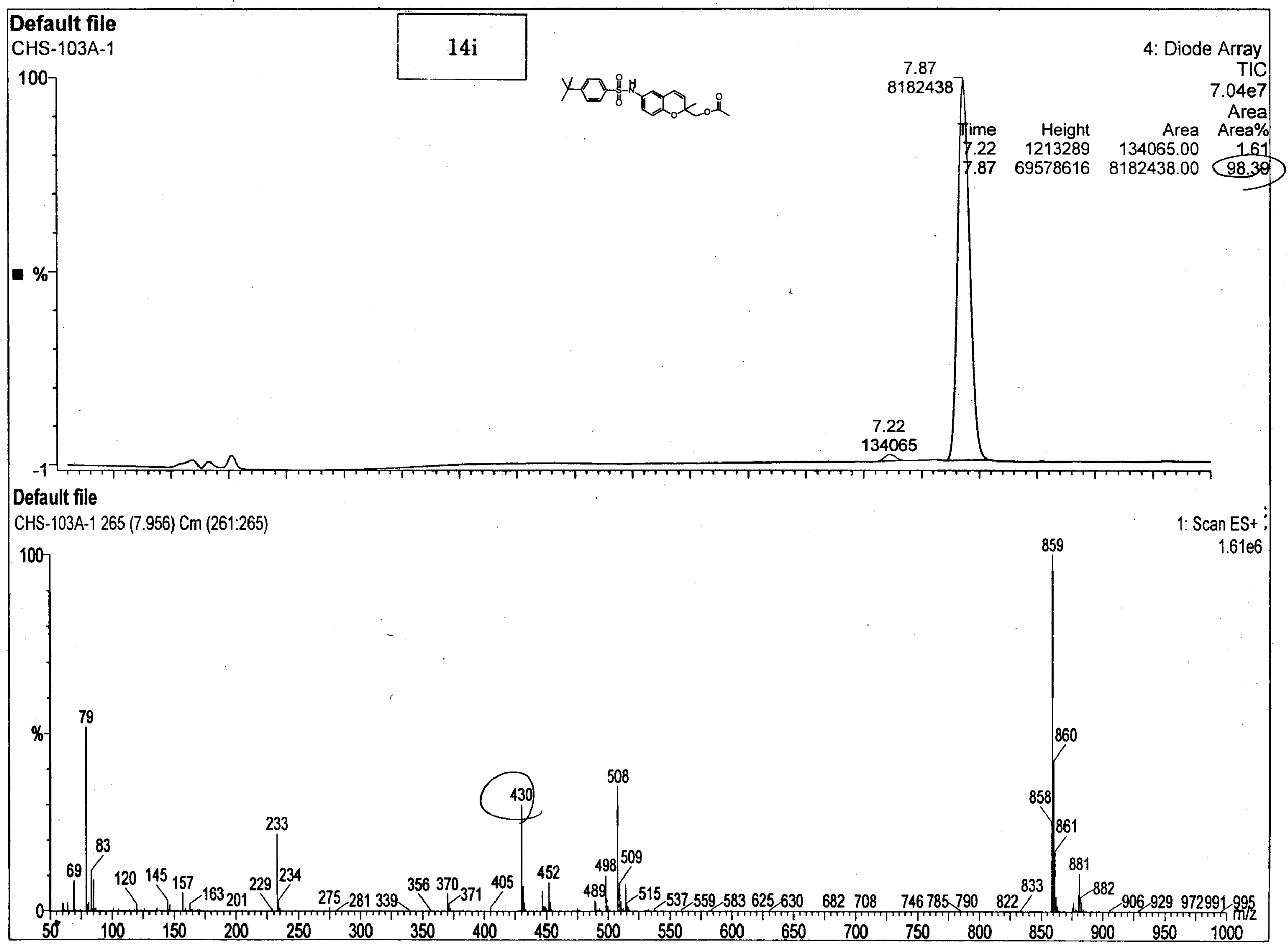



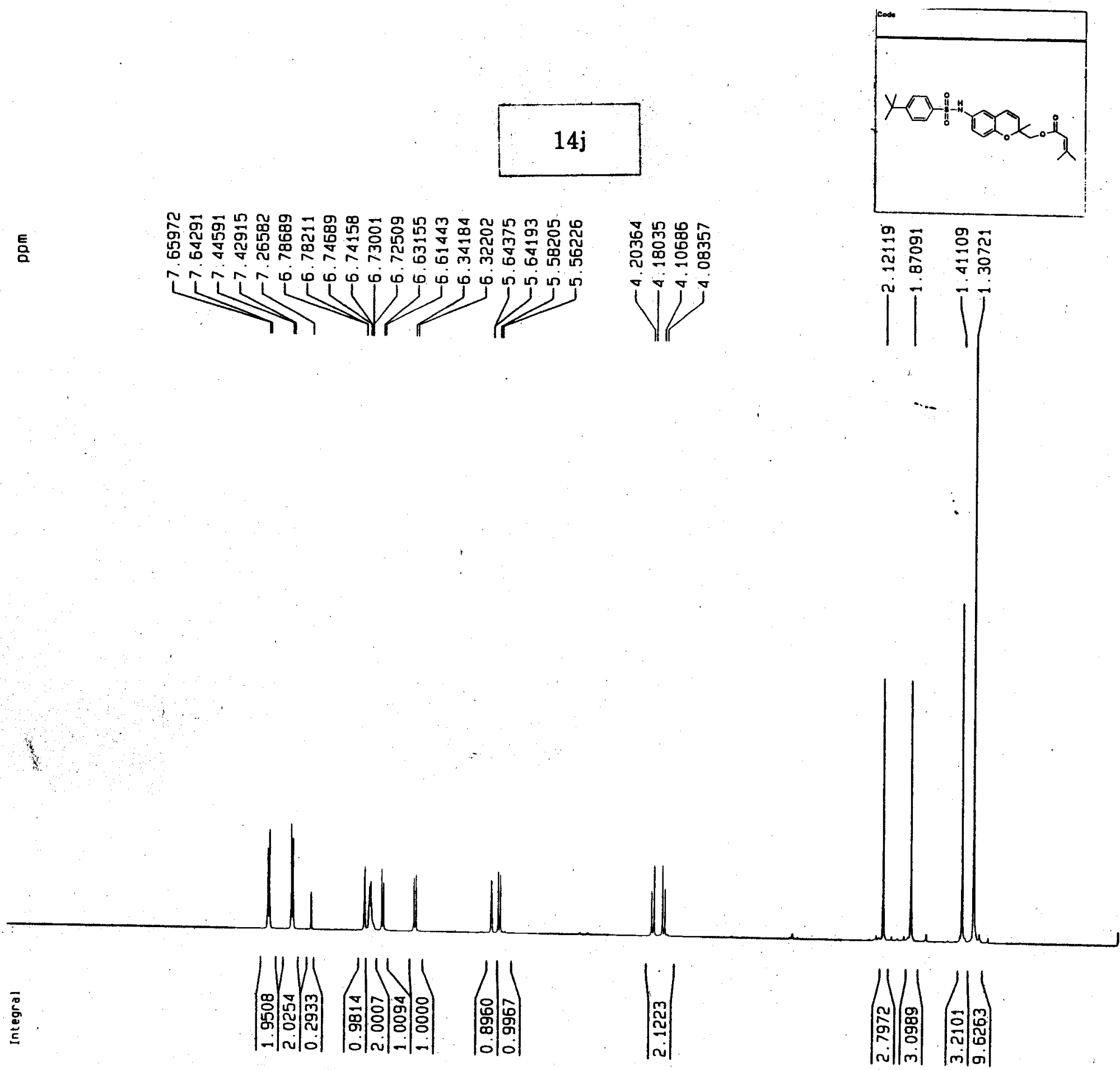


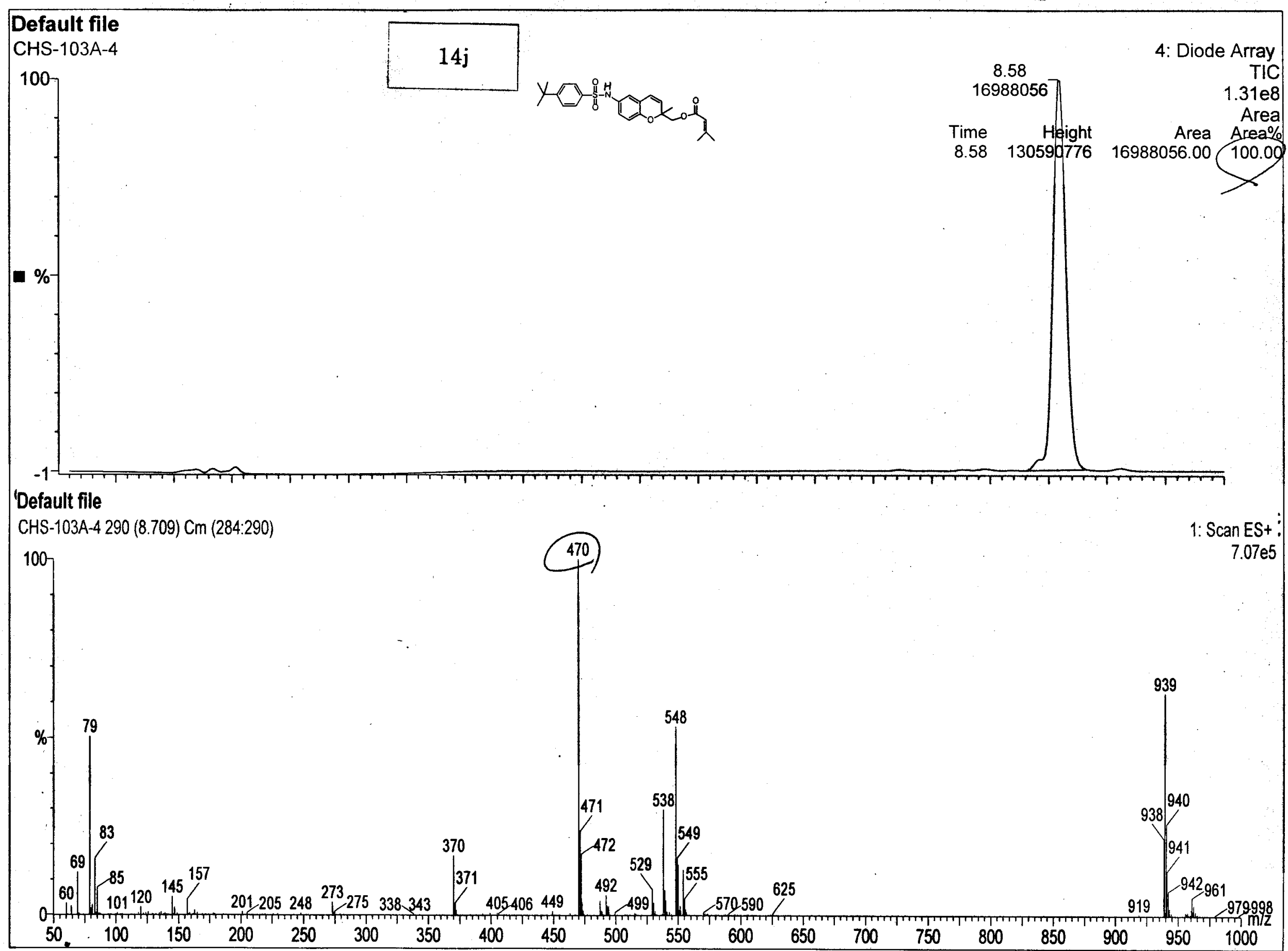




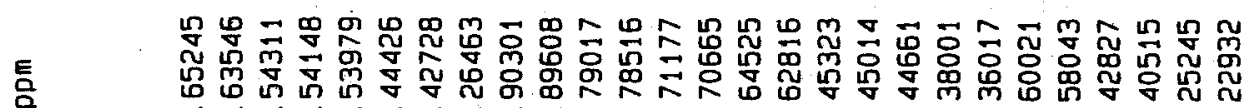

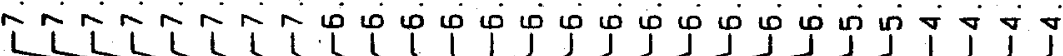
(1)

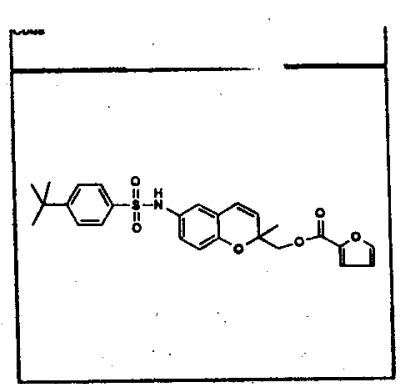

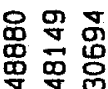
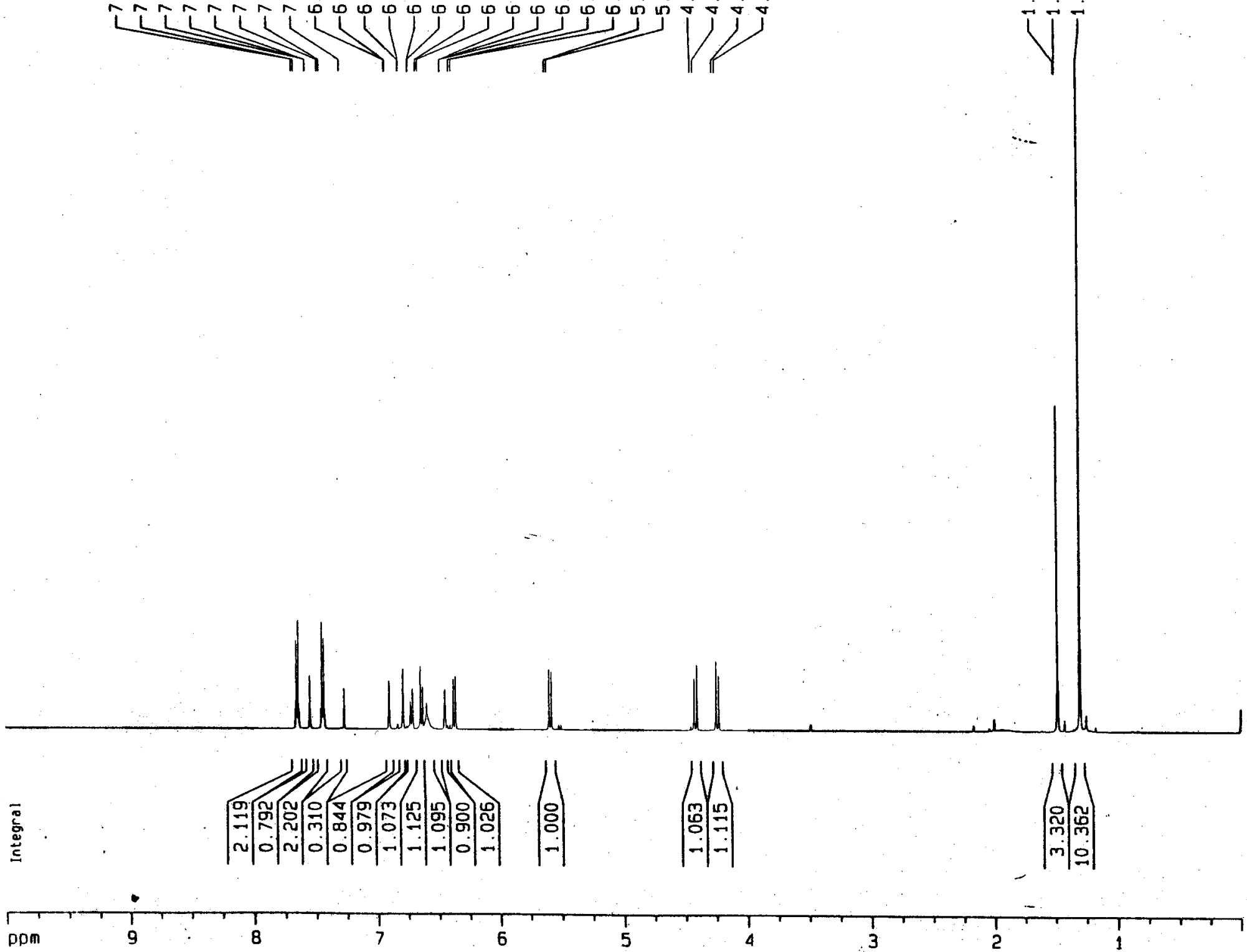

Current Data Parameters

NAME

EXPNO

F2 - Acquisition Parameters

Date 20041115

Time 23.18

INSTRUM spect

PAOBHD $5 \mathrm{~mm}$ DUL $13 \mathrm{C}-1$

PULPROG

TO

5OL VENT

NS

DS .

SWH $\quad 0$

SHH $\quad 7507.507 \mathrm{~Hz}$

FIORES $\quad 0.114555 \mathrm{~Hz}$

AQ 4. $3648143 \mathrm{sec}$

AW $\quad 66.600$ usec

DE $\quad 6.00$ use

IE $0.0 \mathrm{~K}$

D1 $\quad 1.00000000 \mathrm{sec}$

MCREST $\quad 0.00000000 \mathrm{sec}$

MCWAK $\quad 0.01500000 \mathrm{sec}$

$== \pm=== \pm$ CHANNEL

NUC1

P! $\quad 10.00$ usec

14

$-2.00 \mathrm{~dB}$

Fe - Processing

5I Processing parameters

SI 32768

SF $\quad 500.1300116 \mathrm{MHz}$

WOW EM

SSB

LB

$\mathrm{PC}$

$\mathrm{EM}$
0.
$0.30 . \mathrm{Hz}$
0.0

0.30.

10 NMP plot parameters

$\begin{array}{ll}\mathrm{CX} & 20.00 \mathrm{~cm} \\ \mathrm{CY} & 20.00 \mathrm{~cm}\end{array}$

$\begin{array}{ll}\text { FIP } & 20.00 \mathrm{~cm} \\ \text { FI } & 10.000 \mathrm{pDm}\end{array}$

$F_{1} \quad 5001.30 \mathrm{~Hz}$

FP $\quad 0.000 \mathrm{pDM}$

$\begin{array}{ll}\mathrm{F} 2 & 0.00 \mathrm{~Hz}\end{array}$

$\begin{array}{lr}\text { PPMCM } & 0.50000 \mathrm{pDm} / \mathrm{Cm} \\ \text { HZCM } & 250.05500 \mathrm{~Hz} / \mathrm{Cm}\end{array}$ 


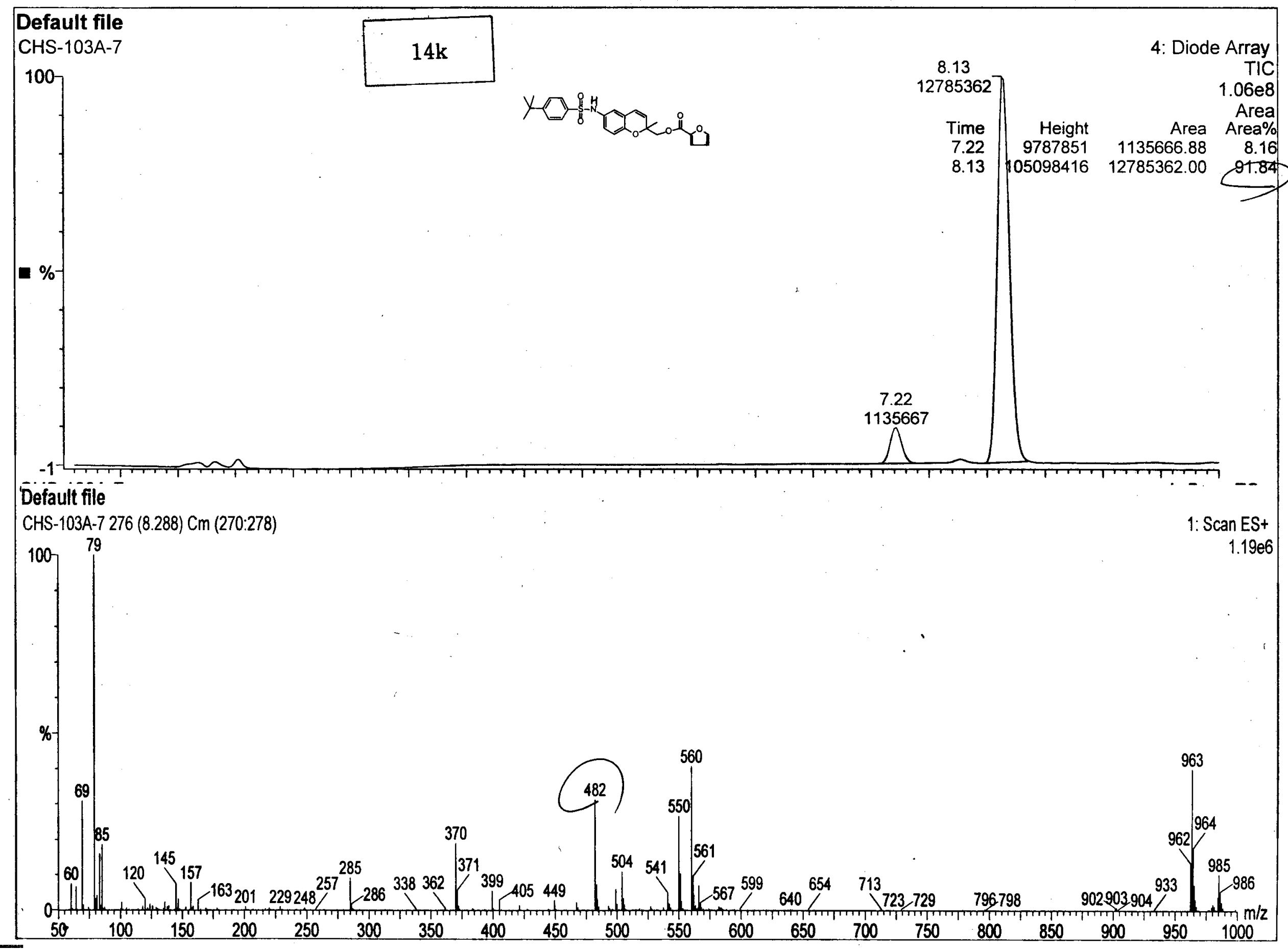




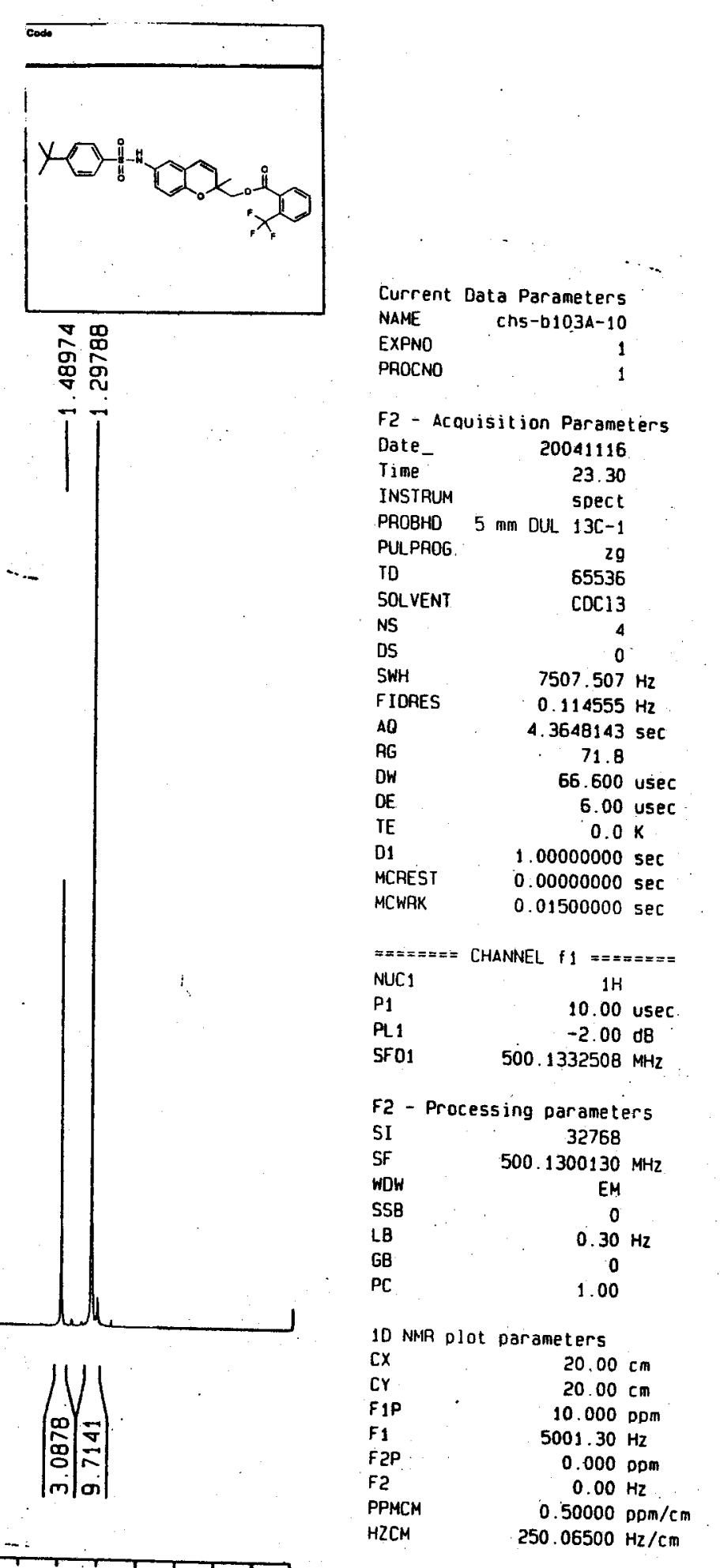

\section{S144}

ฐ

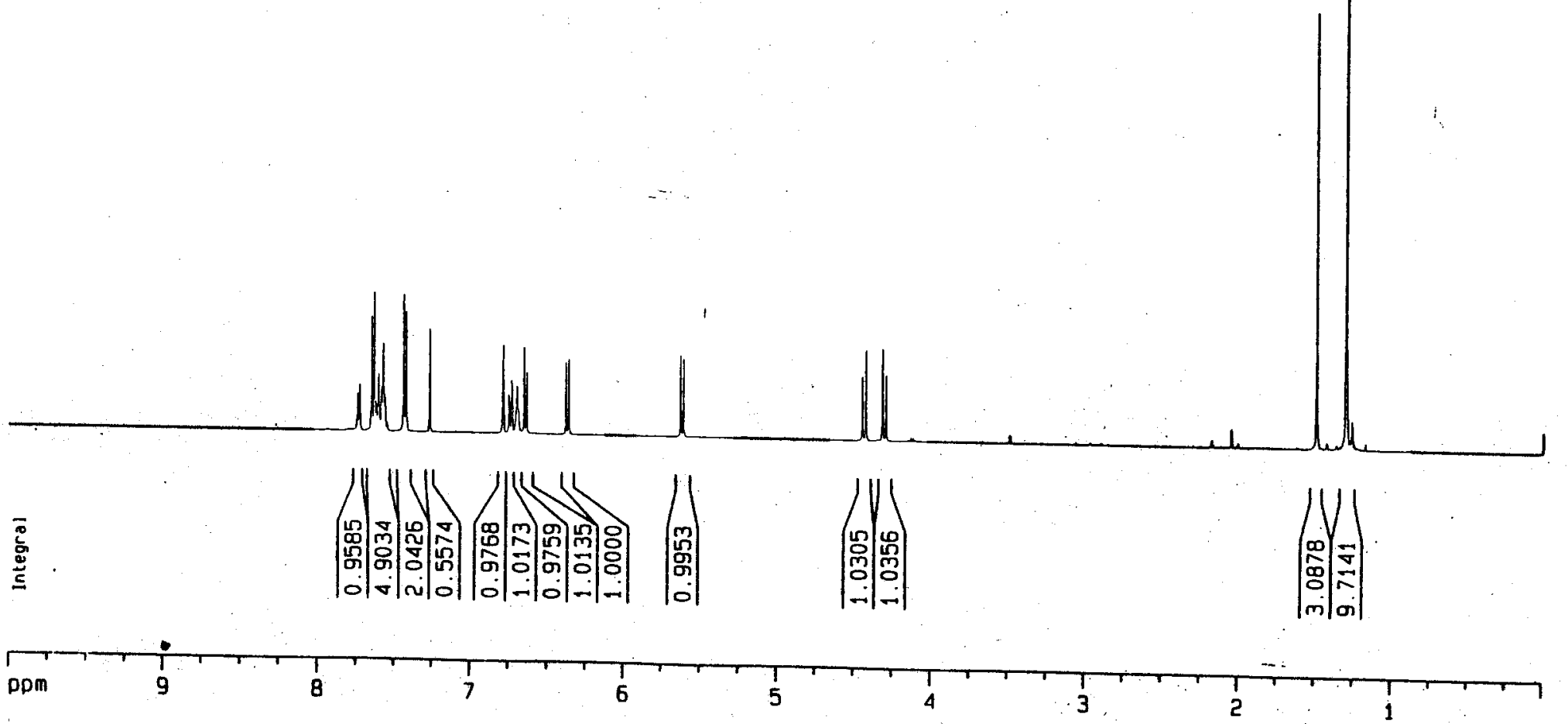




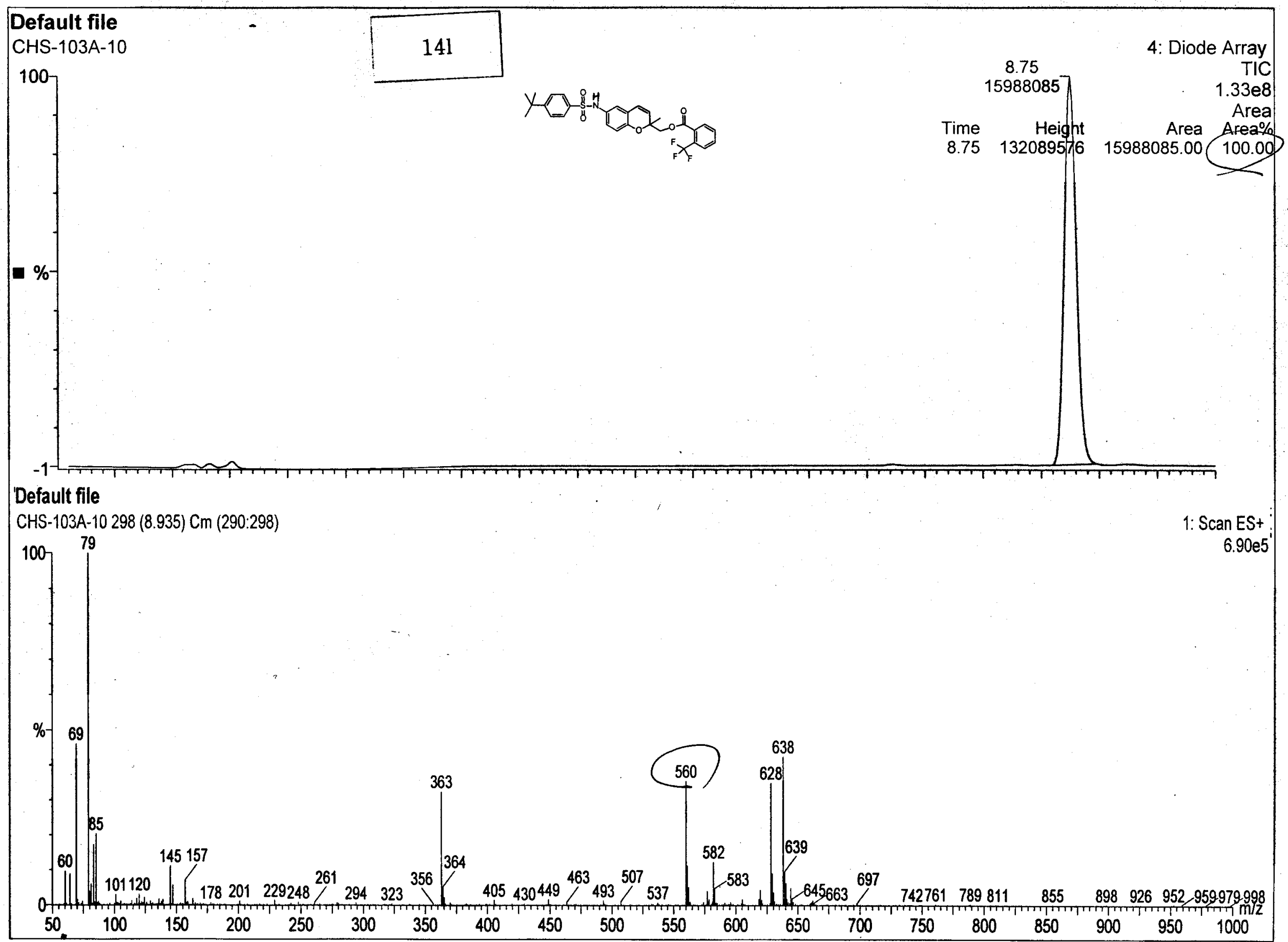




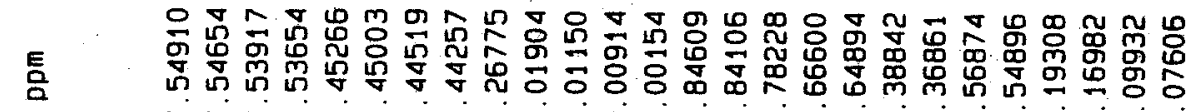
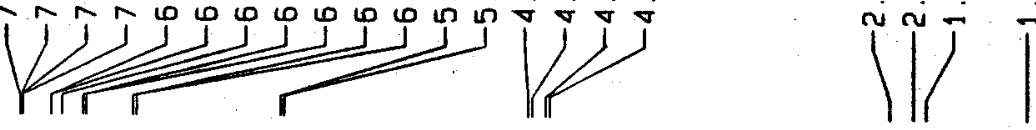

Current Data Parameters

PROCNO

chs-b107A-

F2 - Acquisition Parameters

Date_ 20041123

$\begin{array}{lr}\text { Time- } & 22.19\end{array}$

INSTRUM spect

PROBHD $5 \mathrm{~mm}$ DUL $13 \mathrm{C}-1$

PULPROG

$\begin{array}{lr}\text { PULPROG .. } & 29 \\ \text { ID } & 65535\end{array}$

SOLVENT COCI3

NS

$\begin{array}{lr}\text { DS } & 0 \\ \text { SWH } & 7507.507\end{array}$

FIDRES $\quad 0.114555 \mathrm{~Hz}$

AO 4. 3648143 $\mathrm{sec}$

RG 64

OW 66.600 usec

DE 6.00 use

MCPEST $\quad 1.0000000 \mathrm{sec}$

MCREST $0.00000000 \mathrm{sec}$

$0.01500000 \mathrm{sec}$

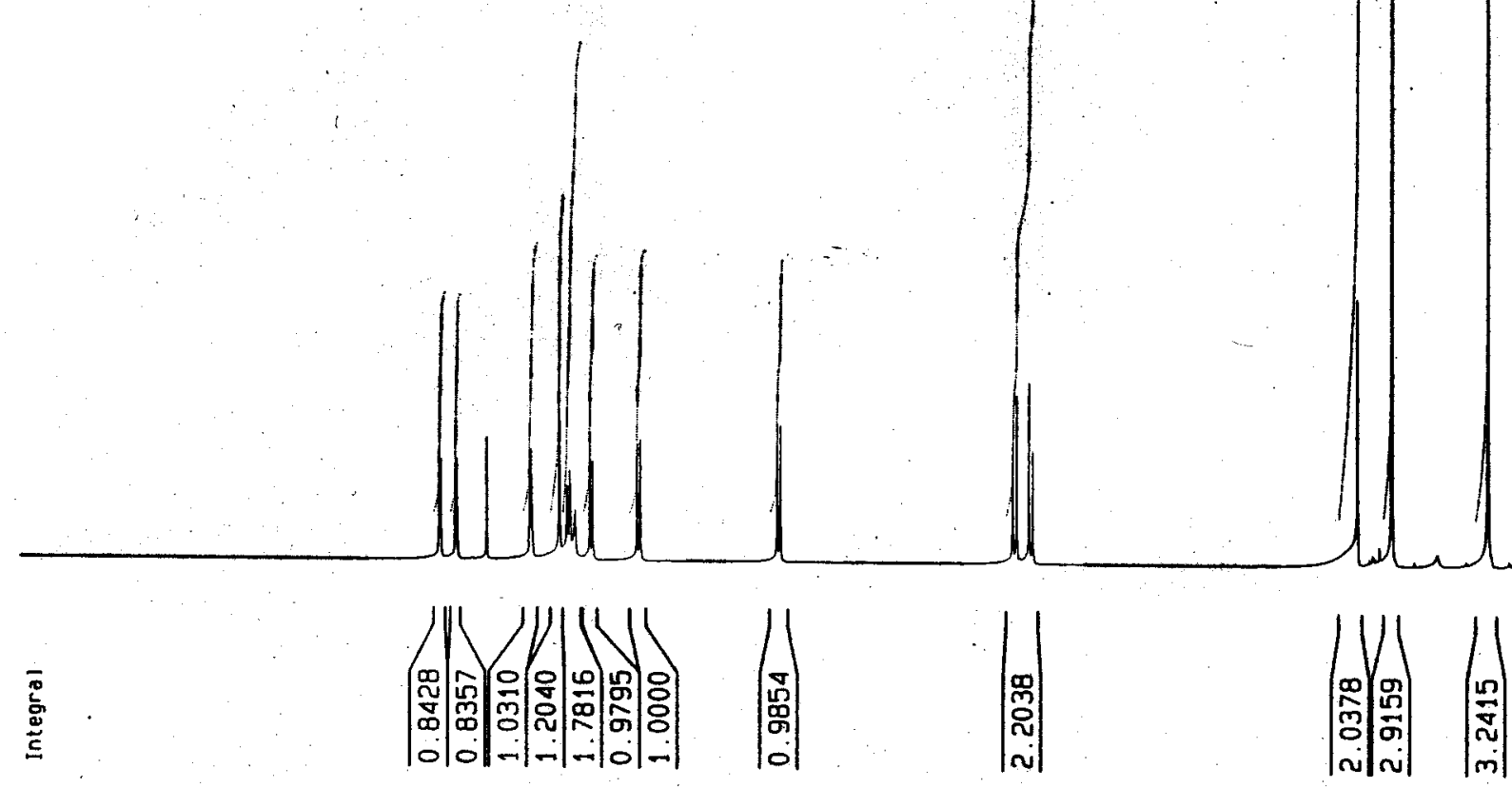

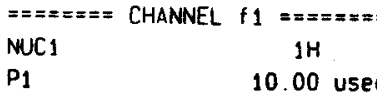

$P_{1} \quad 10.00$ usec

$\begin{array}{lr}P L 1 & -2.00 \mathrm{~dB} \\ \mathrm{SF} 01 & 500.1332508 \mathrm{BHz}\end{array}$

F2 - Processing parameters

SI 32768

SF $\quad 500.1300099 \mathrm{MHZ}$

WOW $\quad 500.1300099$

SSB 0

LB $\quad 0.30 \mathrm{M}$

$\begin{array}{ll}\mathrm{GB} & \\ \mathrm{PC} & \end{array}$

10 NMR plot parameters

CX $\quad 20.00 \mathrm{~cm}$

CY $\quad 10.00 \mathrm{~cm}$

$\begin{array}{lr}F_{1 P} & 10.000 \mathrm{pom} \\ F_{1} & 5001.30 \mathrm{~Hz}\end{array}$

F2P $-1.030 \mathrm{POm}$

F2 $\quad-515.16 \mathrm{~Hz}$

PPMCM $\quad 0.55150 \mathrm{Dpm} / \mathrm{Cm}$

$\mathrm{HZCM} \quad 275.82297 \mathrm{~Hz} / \mathrm{Cm}$ 


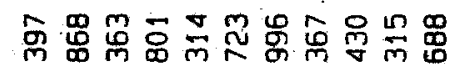

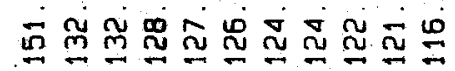

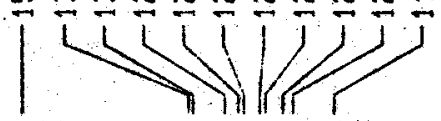

$14 \mathrm{~m}$

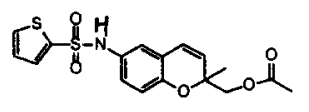

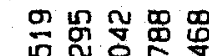

却定果

41

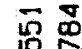

กㅇ

11
Urrent Data Parameters

VAME

JROCNO

Chs-b107A-1

-2 - Acquasition Parameters

jate 20050712

INSTRUM 11.39 JRGBHO 5 min Our $\begin{array}{r}\text { spect } \\ 13 C-1\end{array}$ गपLPR06 2909

$\begin{array}{ll}\text { TE } & 65536 \\ \text { SOLVENT } & \operatorname{COC} 13\end{array}$

SOLVENT
JS

$35211270^{2}$

$35211.270 \mathrm{~Hz}$
$=$ IDPES
$0.537281 \mathrm{~Hz}$

$40 \quad 0.9306754 \mathrm{sec}$

论 $\quad \begin{gathered}0.5306754 \\ 101.6\end{gathered}$

14.200 usec
$\mathrm{JE}$
6.00 usec

TE. $\quad 297.1 \mathrm{k}$

a. $00000000 \mathrm{sec}$

Ji1 $\quad 0.03000000 \mathrm{sec}$

JELTA $\quad 1.89999998 \mathrm{sec}$

$0.00000000 \mathrm{sec}$

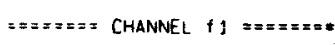

$\begin{array}{ll}\text { vued } & 130 \\ 21 & 7.50\end{array}$

$x_{1}+10008$

5F01 $125.7728799 \mathrm{MHZ}$

$==s= \pm==$ CHANNEL $t 2== \pm===$

CPOPRG2 waltz16

$\begin{array}{ll}\text { WUC2 } & \text { JH } \\ 7 C^{2} & 80.00 \text { us }\end{array}$

$\begin{array}{ll}x_{C P D 2} & 80.00 \text { use } \\ x_{2} 2 & -1.40 \mathrm{OB}\end{array}$

${ }^{2} 12 \quad 16.66 \mathrm{~dB}$

I $13 \quad 25.00 \mathrm{~dB}$

SF02 500.1320005 MH-

$=2$ - processing parameters

$\begin{array}{ll}\text { SI } & 32768 \\ \text { SF } & , 25.7577890\end{array}$

WDW $\$ 25.7577890 \mathrm{MH}$

SSB

GB

PC

10 min ploc orang

CX DJot Darameters

$\begin{array}{lr}\mathrm{Cr} & 9.00 \mathrm{~cm}\end{array}$

Fip $\quad 200.000 \mathrm{ppm}$

$\begin{array}{ll}F 1 & 25151.56 \mathrm{~Hz} \\ =2 \mathrm{P} & -20.000 \mathrm{pom}\end{array}$

$\begin{array}{ll}25 & -20.000 \mathrm{pO} \\ F 2 & -2515.16 \mathrm{~Hz}\end{array}$

PPMCM $\quad 11.00000 \mathrm{ppm} / \mathrm{Cm}$ 


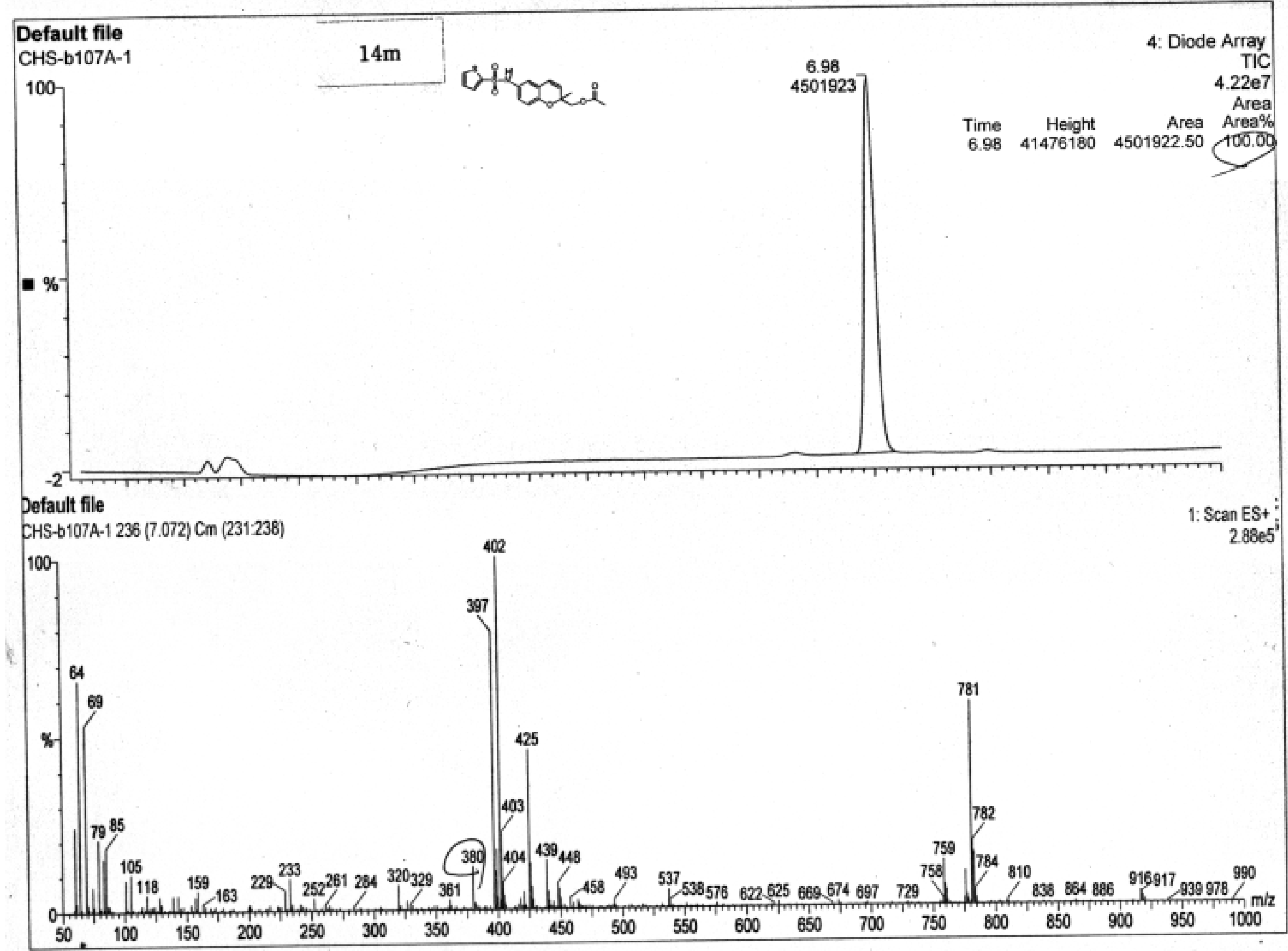


$14 \mathrm{n}$

言

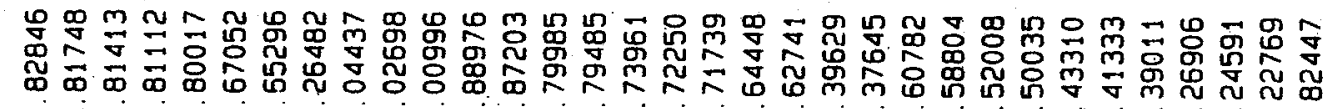

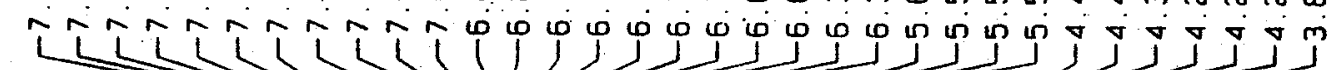

$\rightarrow 11$

.

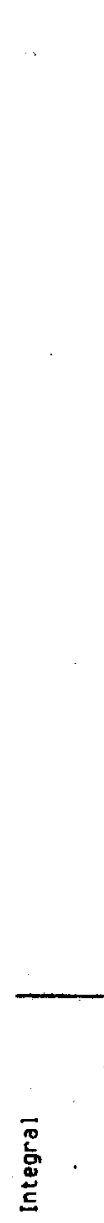

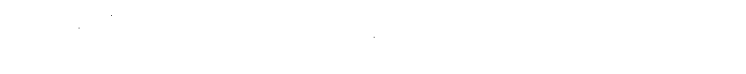

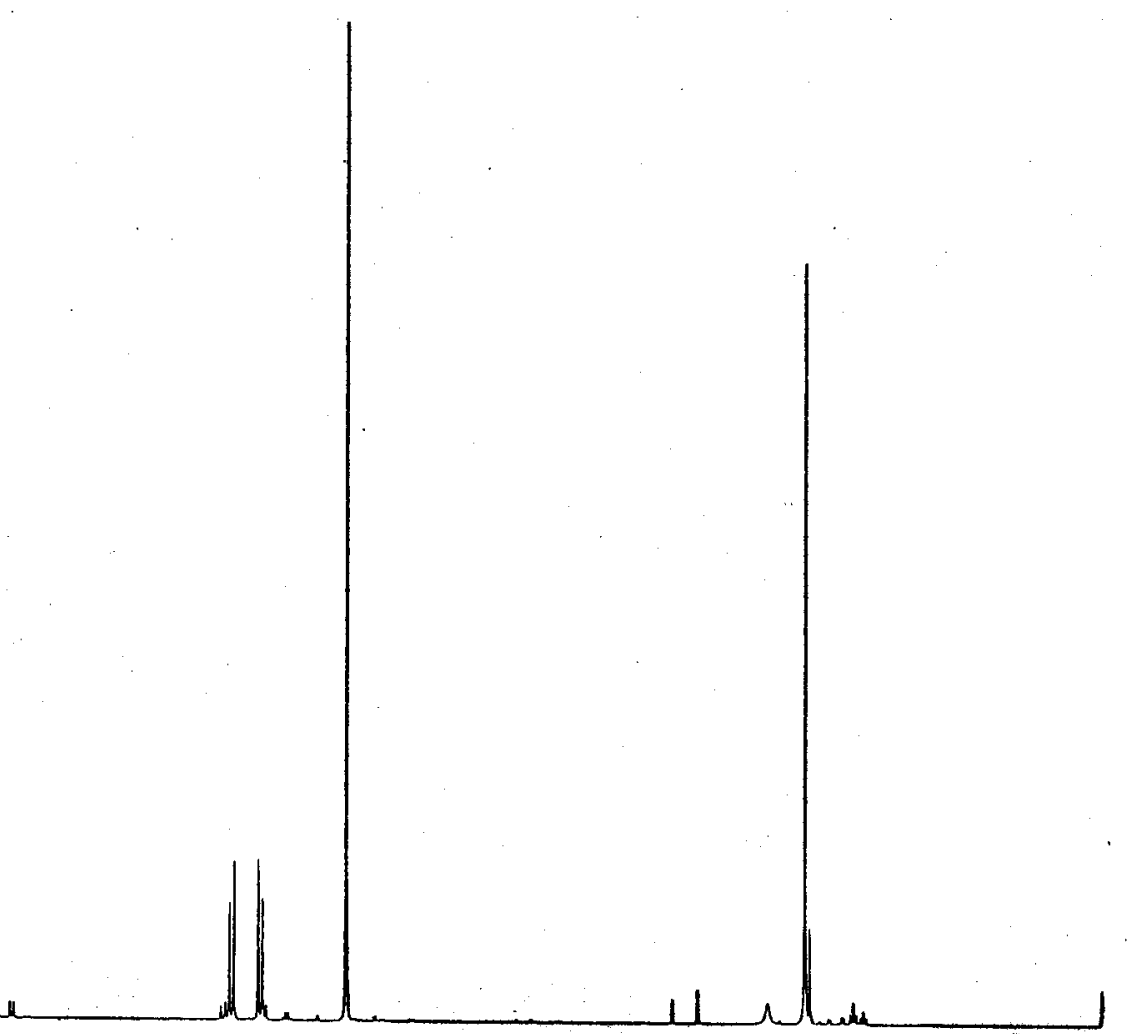

离

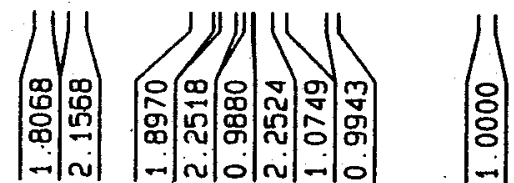

|
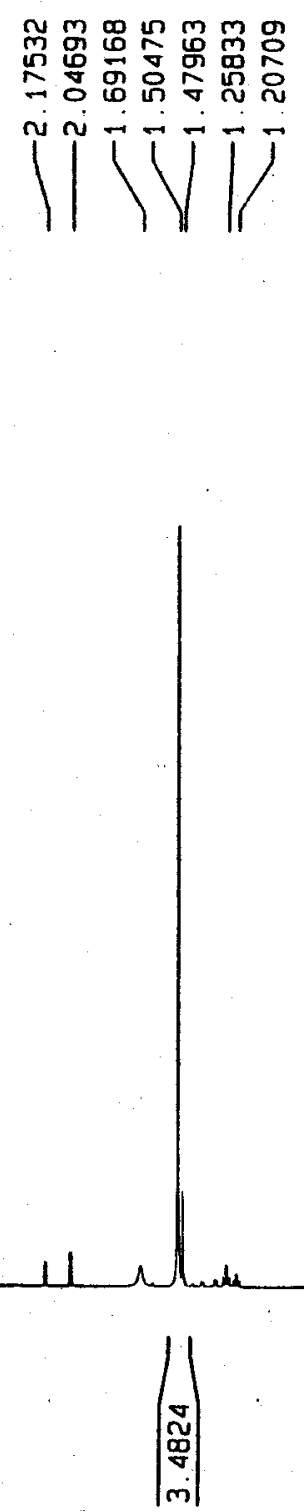

인
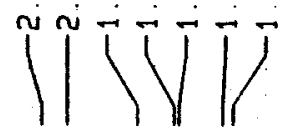


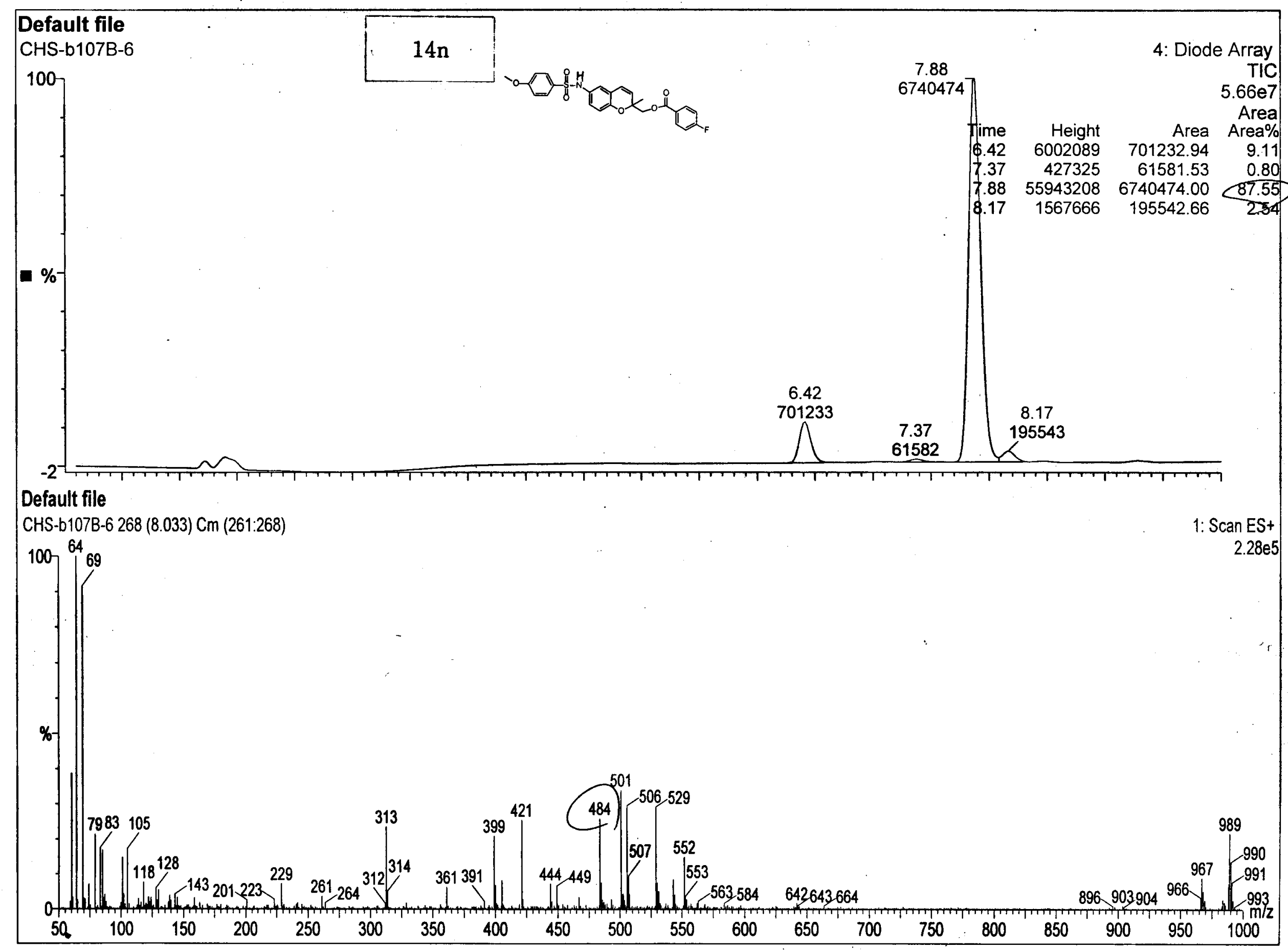




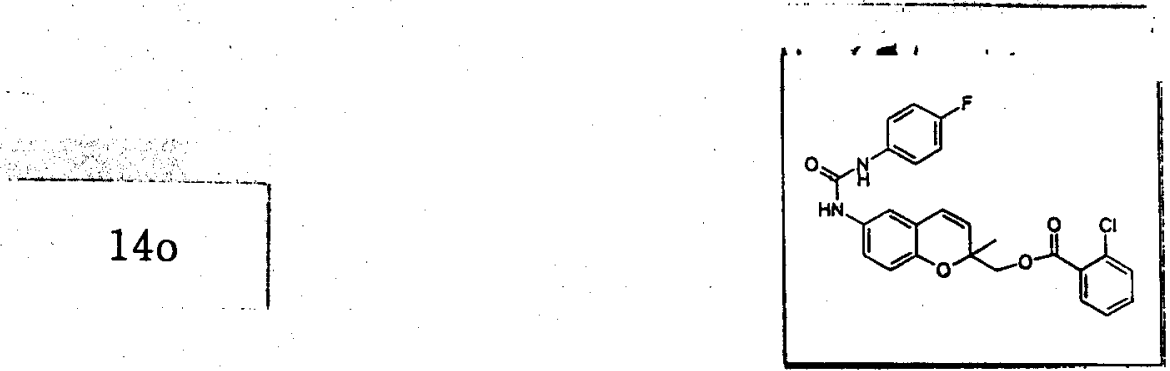

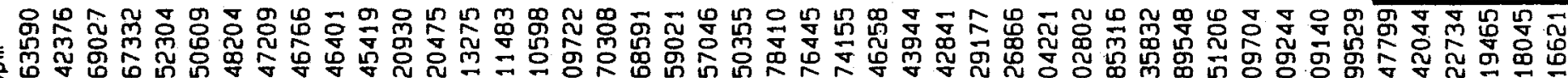

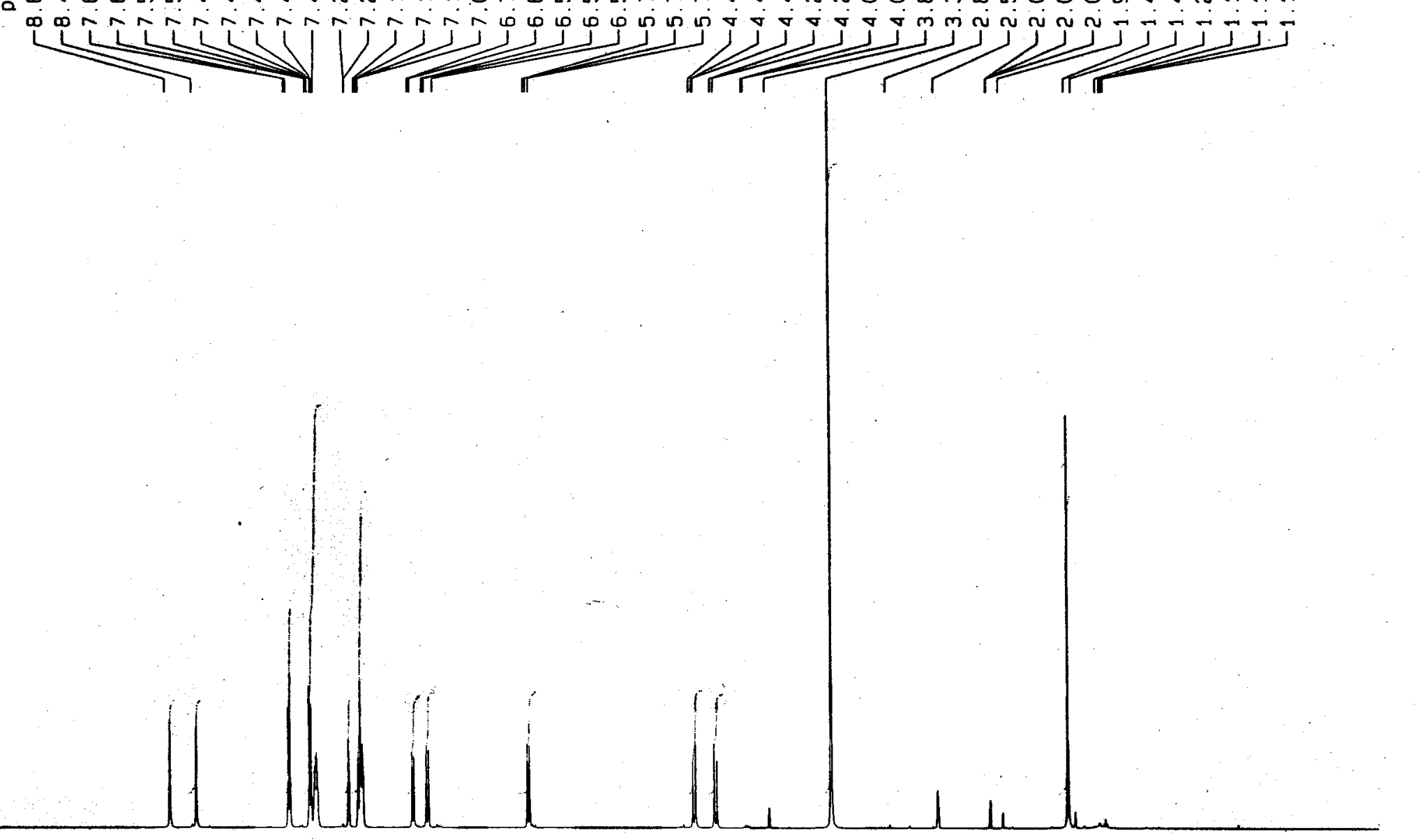

EXPNO

PAOCNO

F2 - Acquisstion Parameters

Date_ 20041102

Time 16.41

INSTRUM

PROBHD $5 \mathrm{~mm}$ DUL $13 C-1$

PULPROG

TD

SOLVENT

NS

SWH $\quad 7507.507 \mathrm{~Hz}$

O.114555 Hz

AQ 4.3648143 sec

$\begin{array}{ll}\text { RG } & 71.8 \\ \text { DW } & 66.600 \text { usec }\end{array}$

DE $\quad 6.00$ usec

TE $0.0 \mathrm{~K}$

$01 \quad 1.00000000 \mathrm{sec}$

MCREST $\quad-0.00000000 \mathrm{sec}$

MCWRK $\quad 0.01500000 \mathrm{sec}$

$==s=z==$ CHANNEL fi $x=z====$

NUC1 $1 \mathrm{H}$

$P_{1} \quad 10.00$ usec

PL1 $\quad-2.00 \mathrm{~dB}$

SF01 $\quad 500.1332508 \mathrm{MHz}$
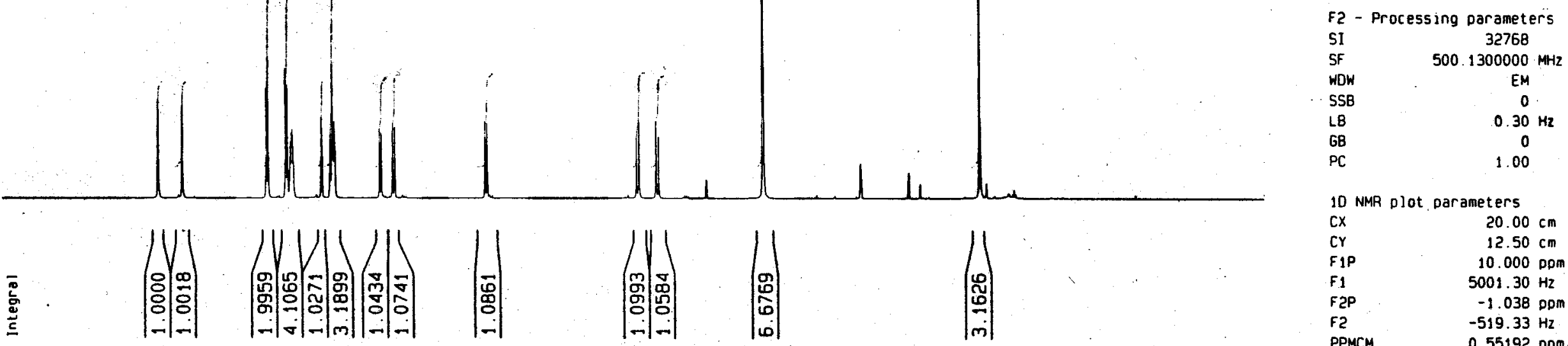

10 NMR plot parameters

CX $\quad 20.00 \mathrm{~cm}$

CY $\quad 12.50 \mathrm{~cm}$

F1P $\quad 10.000 \mathrm{pDm}$

$F_{1} \quad 5001.30 \mathrm{~Hz}$

$\begin{array}{ll}F 2 P & -1.038 \mathrm{Dpm} \\ \mathrm{F2} & -519.33 \mathrm{~Hz}\end{array}$

PPMCM $\quad 0.55192 \mathrm{ppm} / \mathrm{Cm}$

$\mathrm{HZCM} \quad 276.03152 \mathrm{~Hz} / \mathrm{cm}$

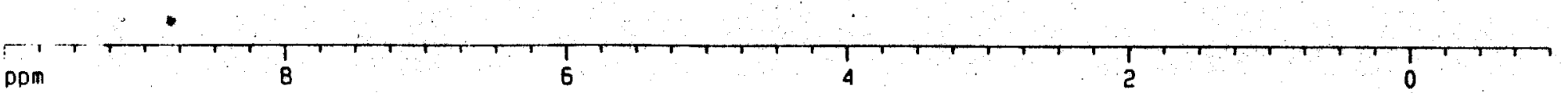




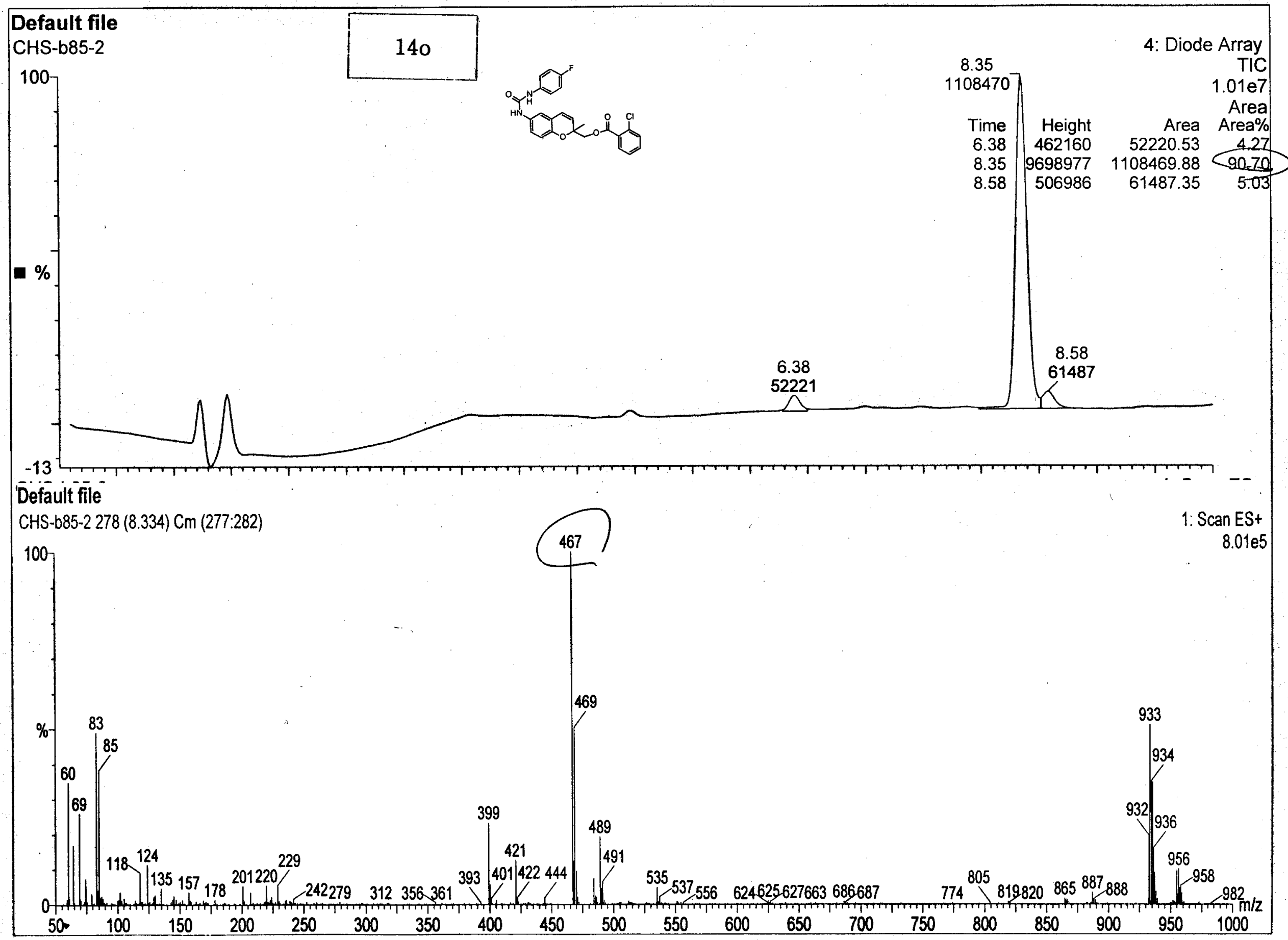




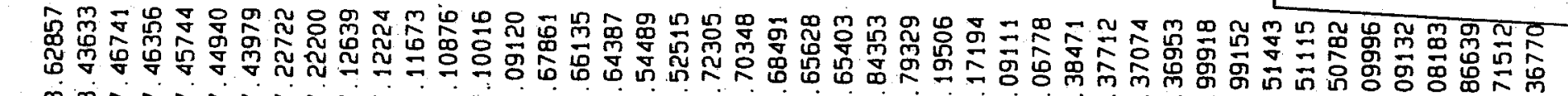

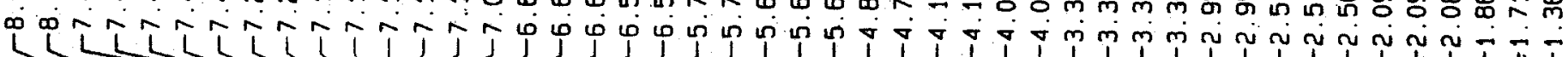
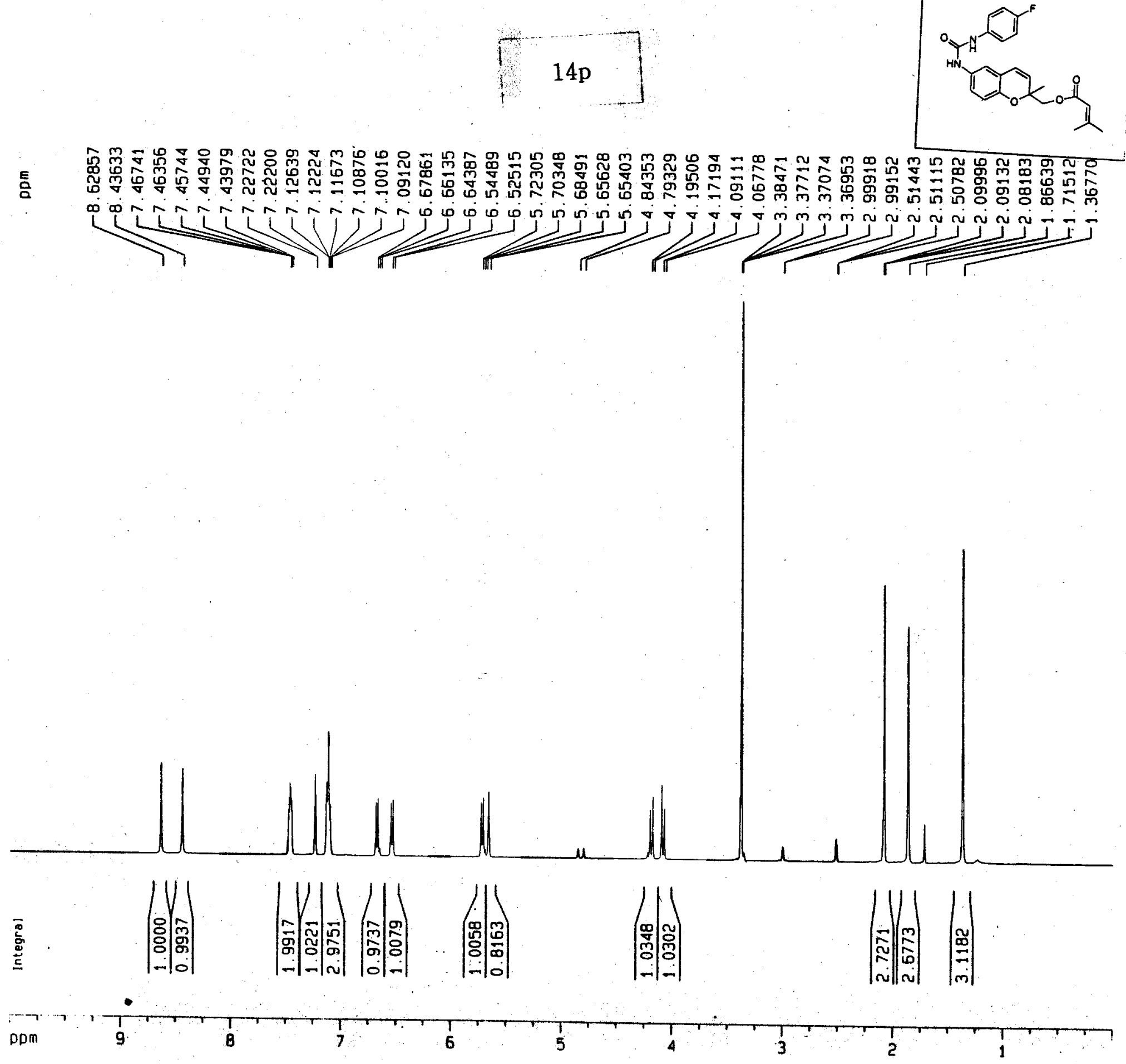

Current Data Parameters

F2 - Acquisition Parameters

Date_ 20041102

ime

17.17

Spect

ID

SOLVENT

SOL

OS

OS

FIDAES

$A O$

RG

DW

DE

IE

D1

29
65536

MCWRK

$7507.507 \mathrm{~Hz}$

$0.114555 \mathrm{~Hz}$

4. $3648143 \mathrm{sec}$ 57

66.600 usec

6.00 usec

$\quad 0.0000000 \mathrm{sec}$

$=x==x=$ CHANNEL if $x=x=z=x=$

$\begin{array}{ll}\text { NUCA } & 1 \mathrm{H} \\ \text { P1 } & 10.00 \text { usec }\end{array}$

PL1 $\quad-2.00 \mathrm{~dB}$

SF $01 \quad 500.1332508 \mathrm{MHZ}$

F.2 - Processing parameters

32768

SF $\quad 500.1300000 \mathrm{MHZ}$

WDW EM

SSB $\quad 0$

$\begin{array}{rr}\text { LB } & 0.30 \\ G B & 0\end{array}$

PC $\quad 1.00$

10 NMR plot parameters

CX $20.00 \mathrm{~cm}$

$\begin{array}{ll}\mathrm{Cr} & 10.00 \mathrm{~cm}\end{array}$

$\begin{array}{lr}F_{1 P} & 10.000 \mathrm{ppm} \\ F_{1} & 5001.30 \mathrm{~Hz}\end{array}$

F2P $\quad 0.000 \mathrm{DPm}$

F2

PPMCM

$0.00 \mathrm{~Hz}$

$\mathrm{HZCM}$

$0.50000 \mathrm{Dpm} / \mathrm{Cm}$ 


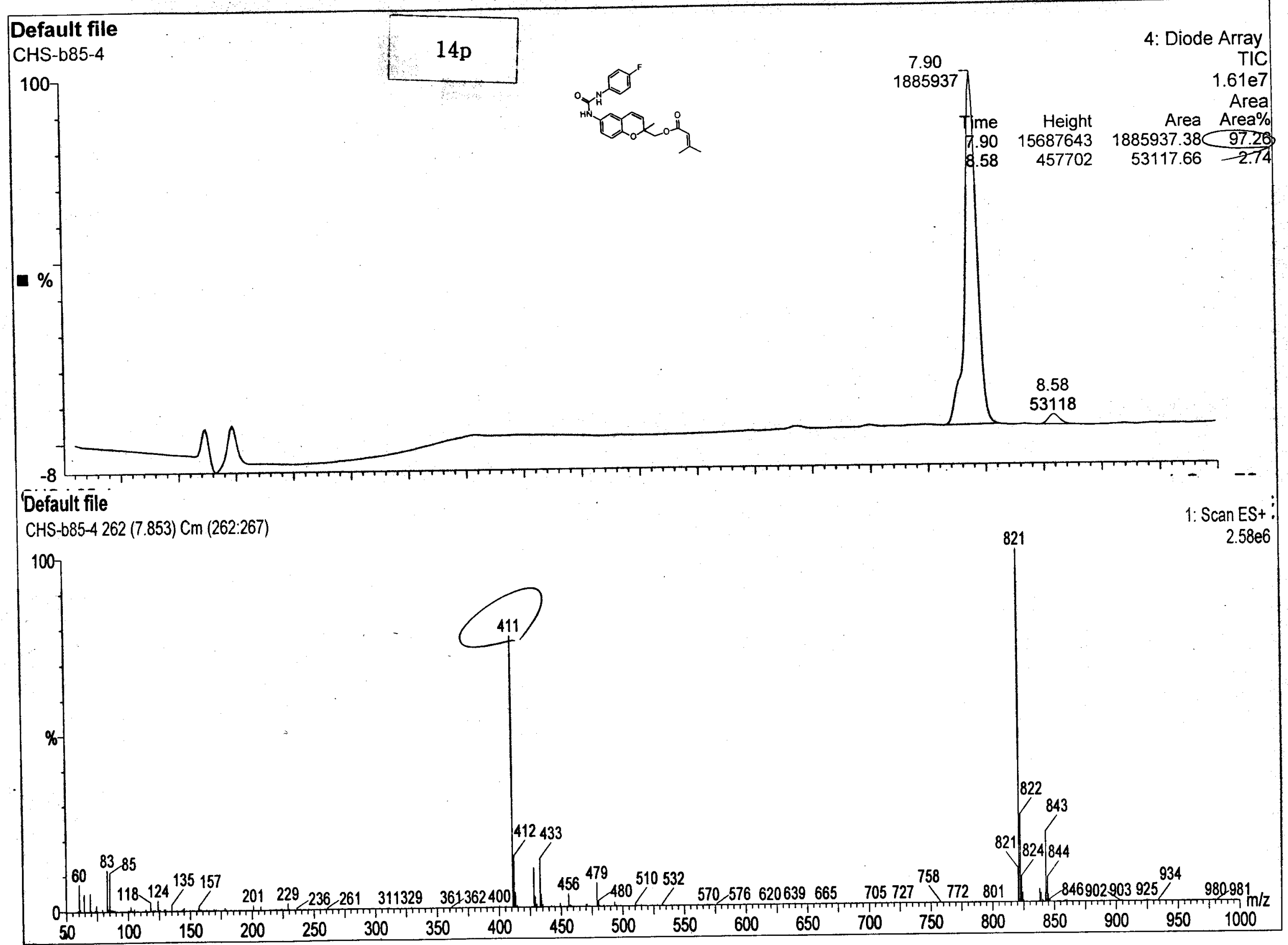




\section{$14 q$}

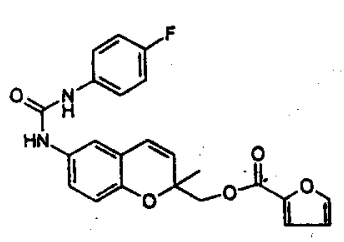

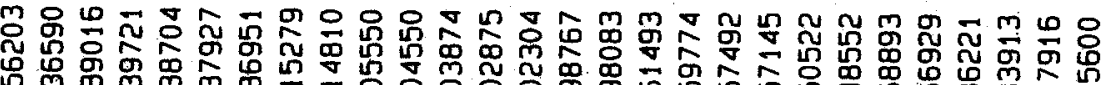

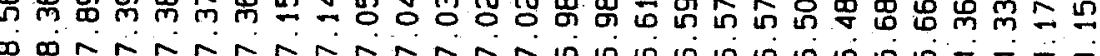

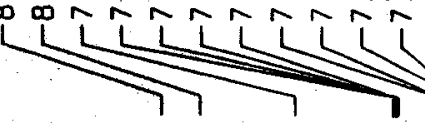
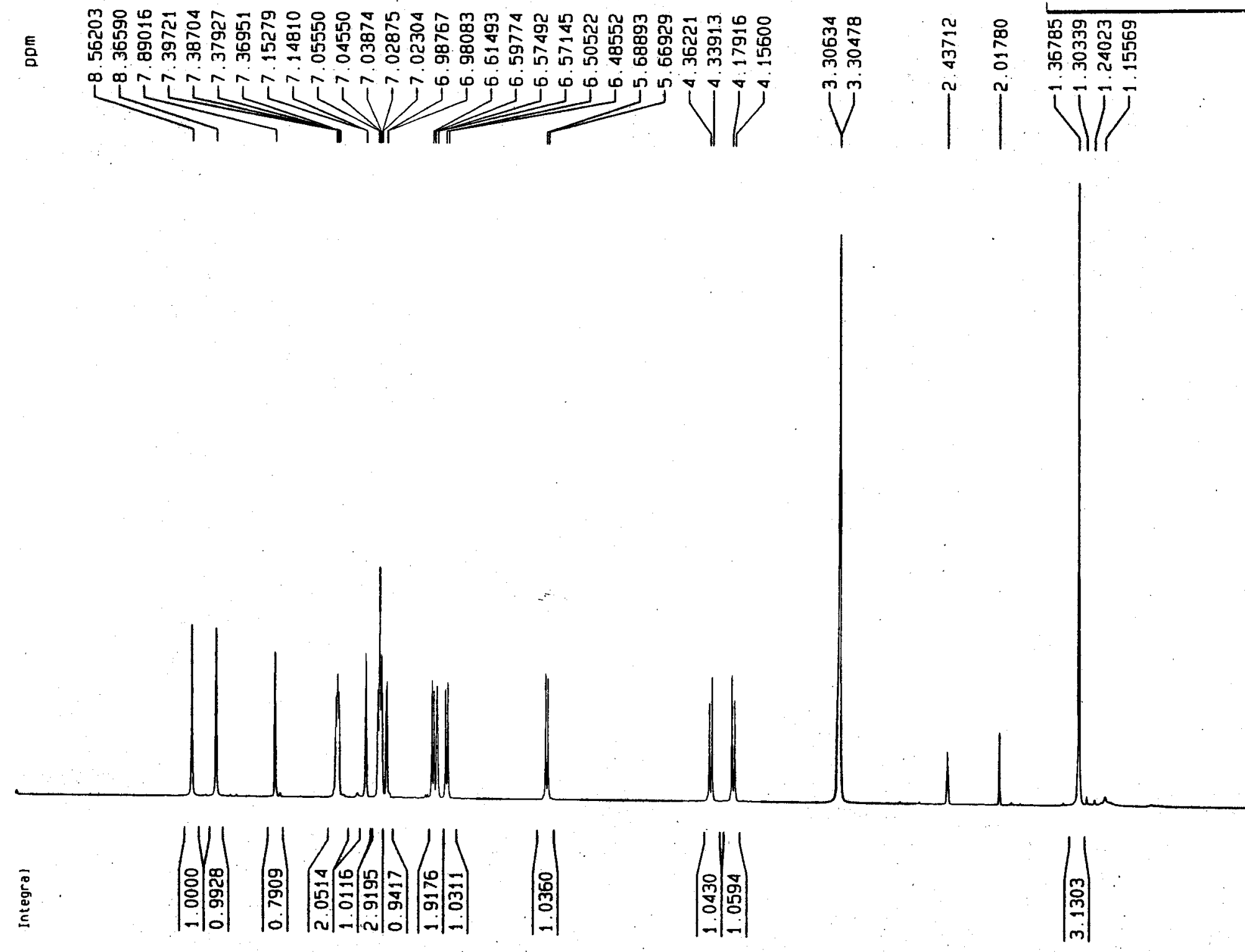


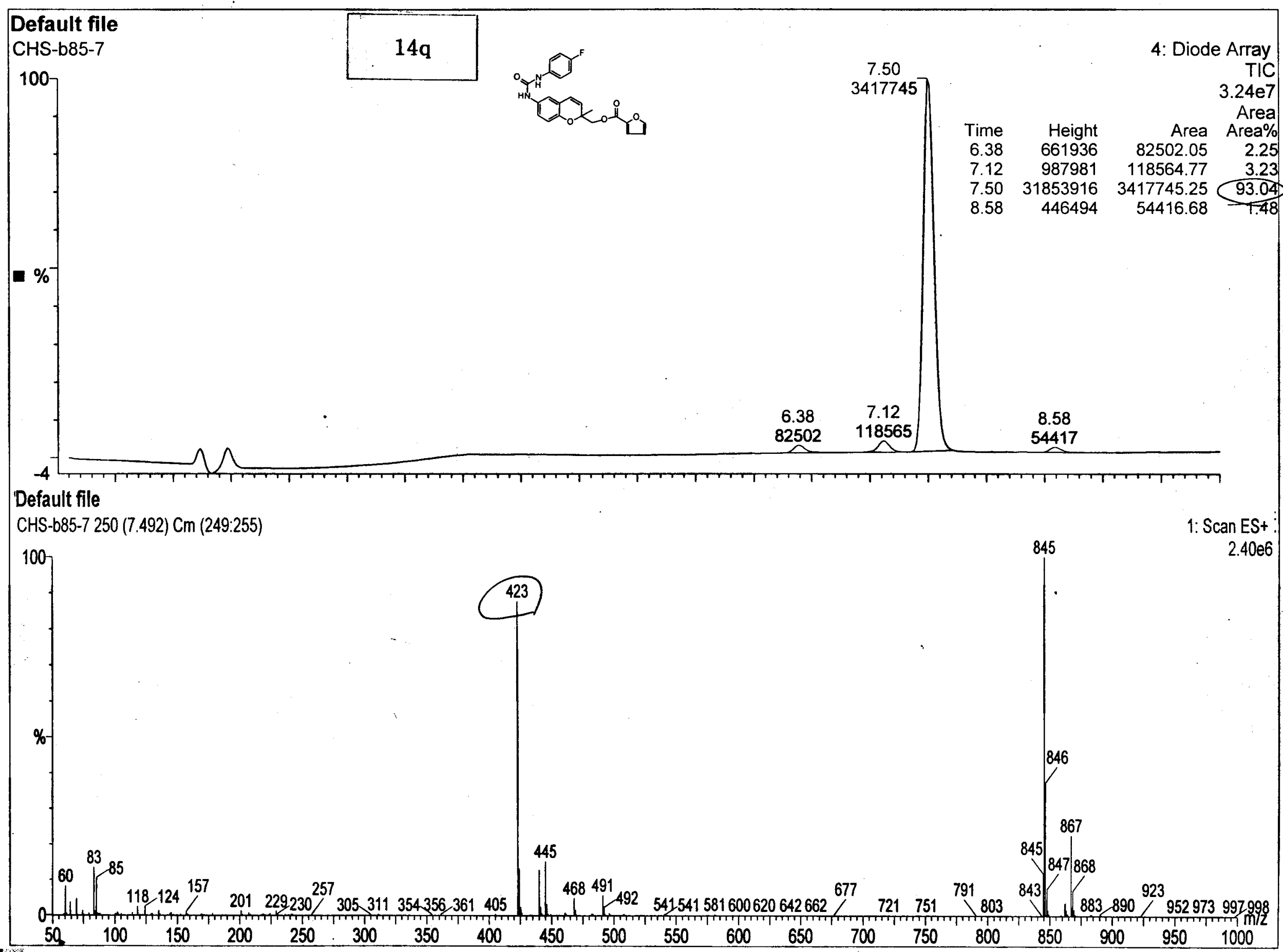




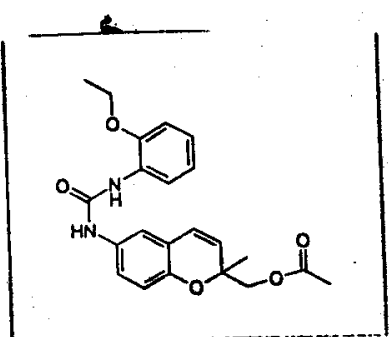

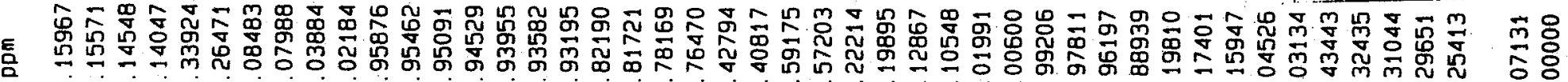

$14 \mathrm{r}$

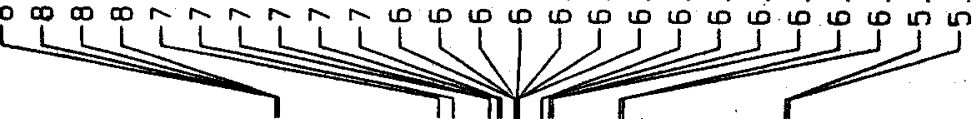

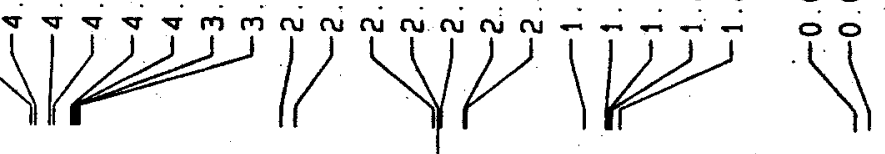

10

NAME chs-b87-1

EXPNO

PROCNO

F2 - Acquisjtion Parameters

Date_ 20041102

Time 20.55

INSIRUM 20.55

PROBHD $5 \mathrm{~mm}$ DUL $13 \mathrm{C}-1$

PROBFD

PULPROG

TD

SOLVENT

DS
SWH
FIDRES

FIDRES

$A Q$
$R G$

RW
DW
DE
TE

TE

D1

MCAEST

29
65536
$\operatorname{CDC} 13$

$\operatorname{CoCl} 3$
4
4

MCWR

$7507.507 \mathrm{~Hz}$

$0.114555 \mathrm{~Hz}$

4. 3648143 SeC

101.6

66.600 usec

6.00 usec

1.00000000

1.0000000 sec

$0.00000000 \mathrm{sec}$

$==x=z=x$ CHANNEL $f 1==s===s$

NUC1

$P_{1}$

PL1 10.00 usec

SF01 $\quad \begin{array}{r}-2.00 \mathrm{~dB} \\ 500.1332508 \mathrm{MHZ}\end{array}$

F2 - Processing Darameters

SI 32768

SF $\quad 500.1300115 \mathrm{MHz}$

SF
WOW
S5B

SSB
LB

LB
68
$P C$

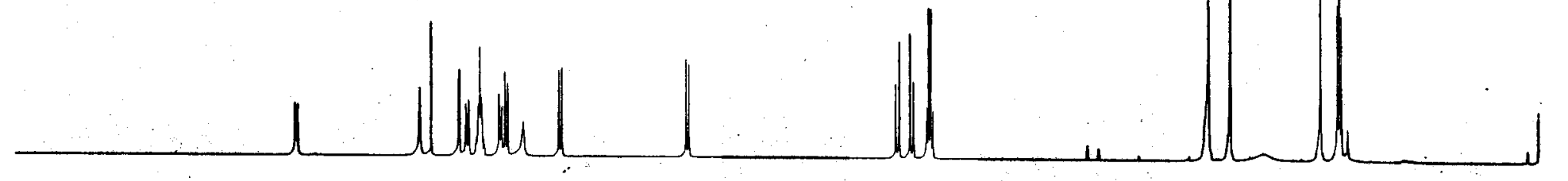

EM

0
$0.30 \mathrm{~Hz}$
0
1.00

1.00

10 NMR pjot parameters

$\begin{array}{ll}\mathrm{CX} & 20.00 \mathrm{~cm} \\ \mathrm{CY} & 15.00 \mathrm{~cm}\end{array}$

$\begin{array}{lr}\text { CY } & 15.00 \mathrm{~cm} \\ \text { F1P } & 10.000 \mathrm{com}\end{array}$

$\mathrm{FI}^{2} \quad 5001.30 \mathrm{~Hz}$

F.P $\quad 0.000 \mathrm{ppm}$

$\mathrm{F2} \quad 0.00 \mathrm{~Hz}$

PPMCM $\quad 0.50000 \mathrm{pom} / \mathrm{cm}$

HZCM

$250.50000 \mathrm{Dpm} / \mathrm{cm}$

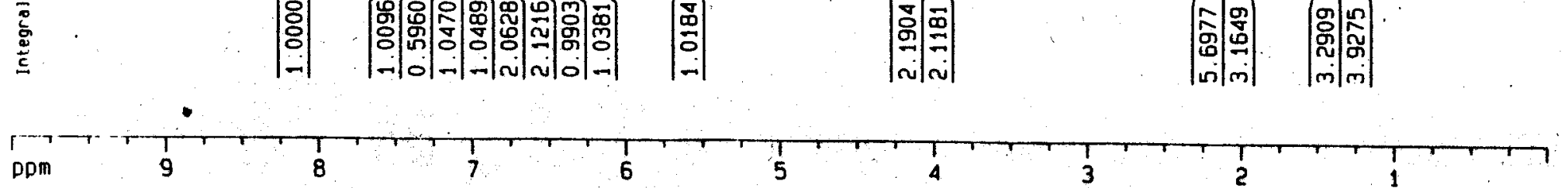




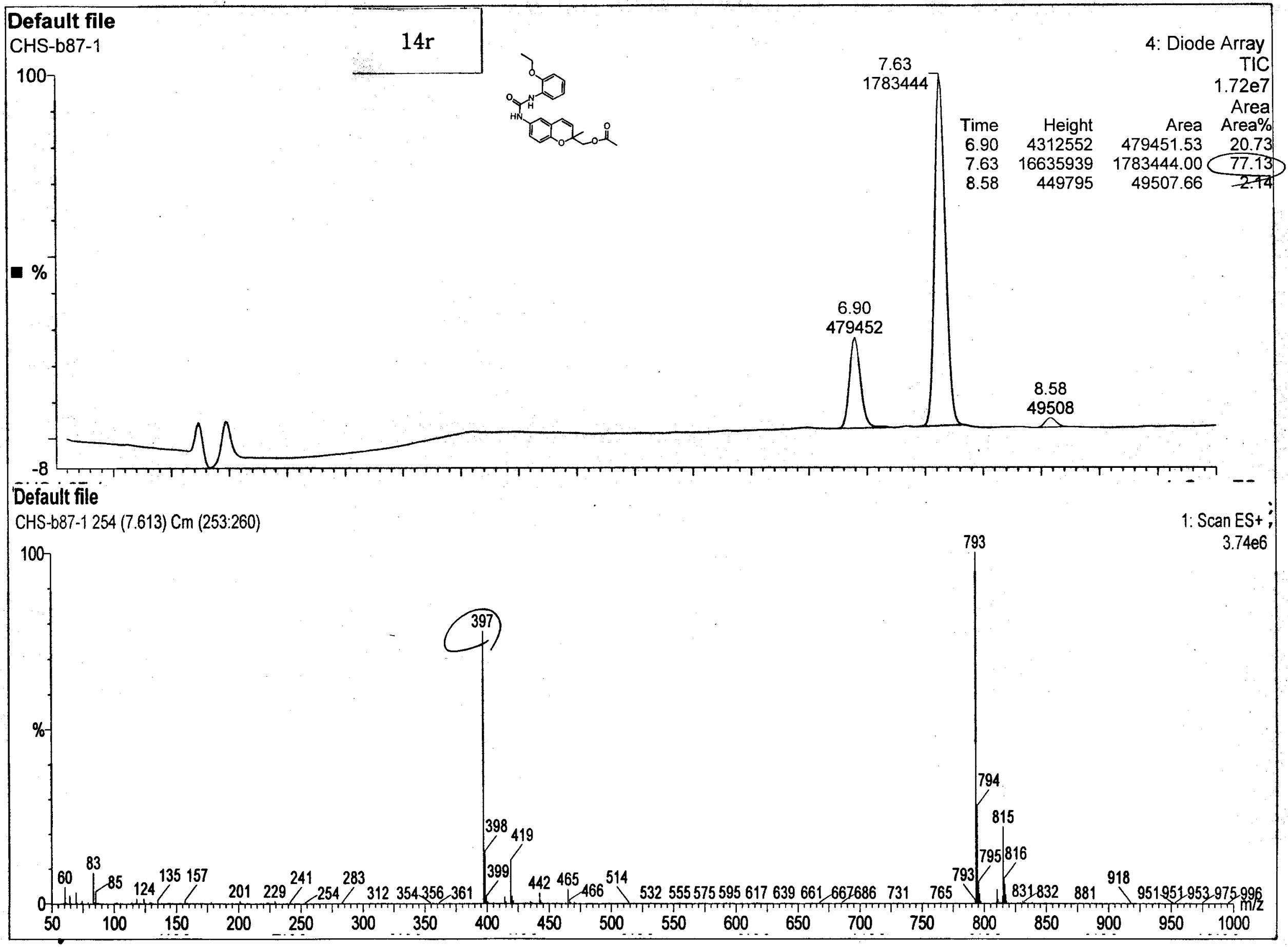




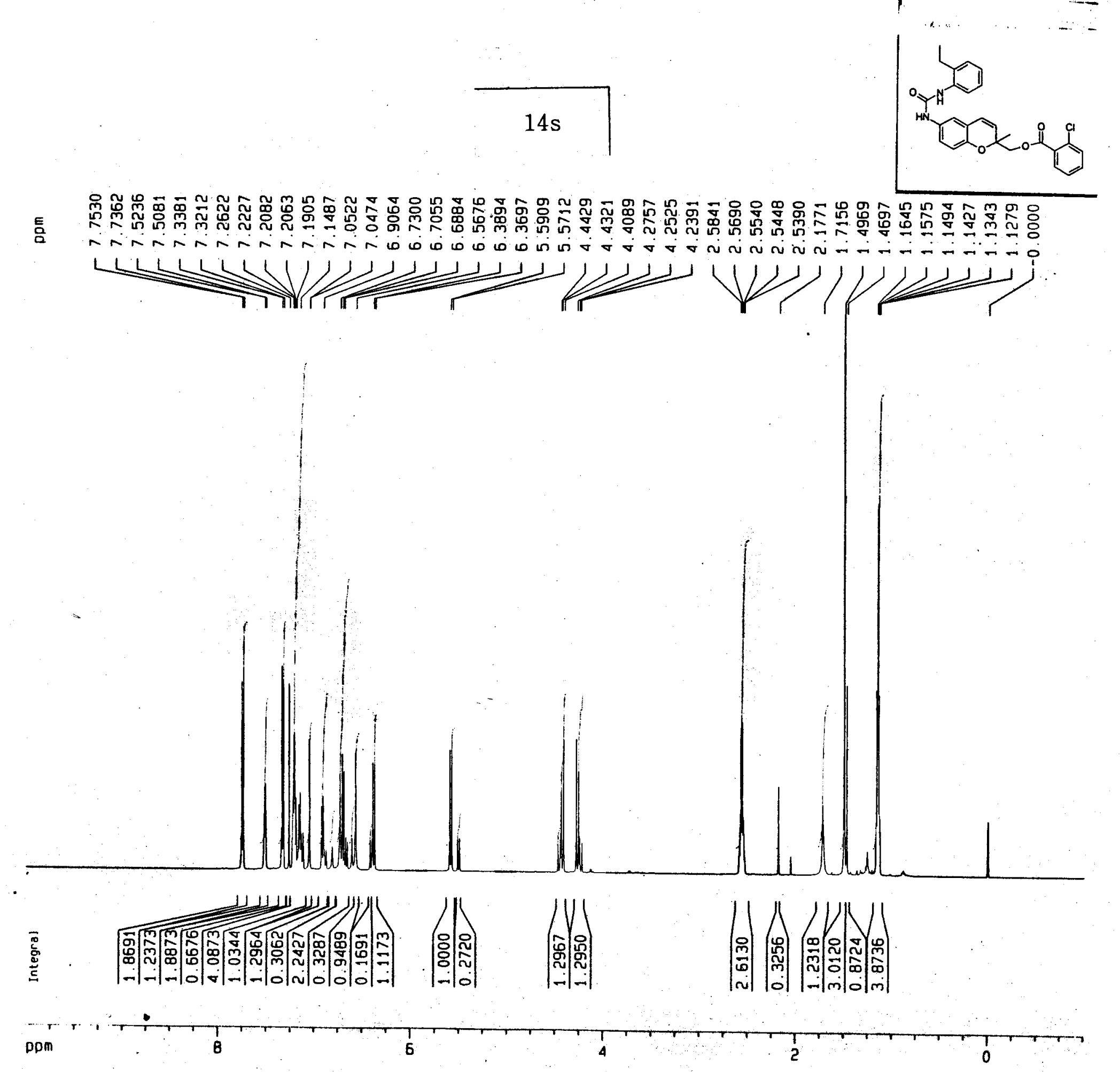

Current Data Parameters

NAME chs-b94a-2

EXPNO

PROCNO

F2 - Acquisitjon Parameters
Date_ 20041109

$\begin{array}{lr}\text { Time } & 23.33 \\ & \end{array}$

INSTRUM spect

PROBHO $5 \mathrm{~mm}$ DUL $13 \mathrm{C}-1$.

PULPROG

$13 C-1$
29
65536

TD

SOLVEN

NS

OS

SWH

$A Q$

AG

DW

OE

IE

DI

MCAEST

$=x==z==$ CHANNEL $i 1 \quad z=z=z=0$

$\begin{array}{ll}\text { NUC1 } & 1 \mathrm{H} \\ \text { P1 } & 10.00 \text { usec }\end{array}$

PL1 $\quad-2.00 \mathrm{~dB}$

SF01 $500.133250 \mathrm{~B}$ MHZ

F2 - Processing parameters

SI 32768

SF $\quad 500.1300126 \mathrm{MHZ}$

WOW EM

SSB

68

$E M$
0
$0.30 \mathrm{~Hz}$

$0.30 \mathrm{~Hz}$
$\mathrm{O}$

1.00

10 NMR plot parameters

$\mathrm{CX} \quad 20.00 \mathrm{~cm}$

$\begin{array}{ll}\mathrm{Cr} & 1200 \mathrm{~cm}\end{array}$

$10.000 \mathrm{Dpm}$

F1 $\quad 5001.30 \mathrm{~Hz}$

F2P $-1.006 \mathrm{ppm}$

$-502: 91 \mathrm{~Hz}$

H2CM $\quad 0.55028 \mathrm{pDm} / \mathrm{cm}$ 


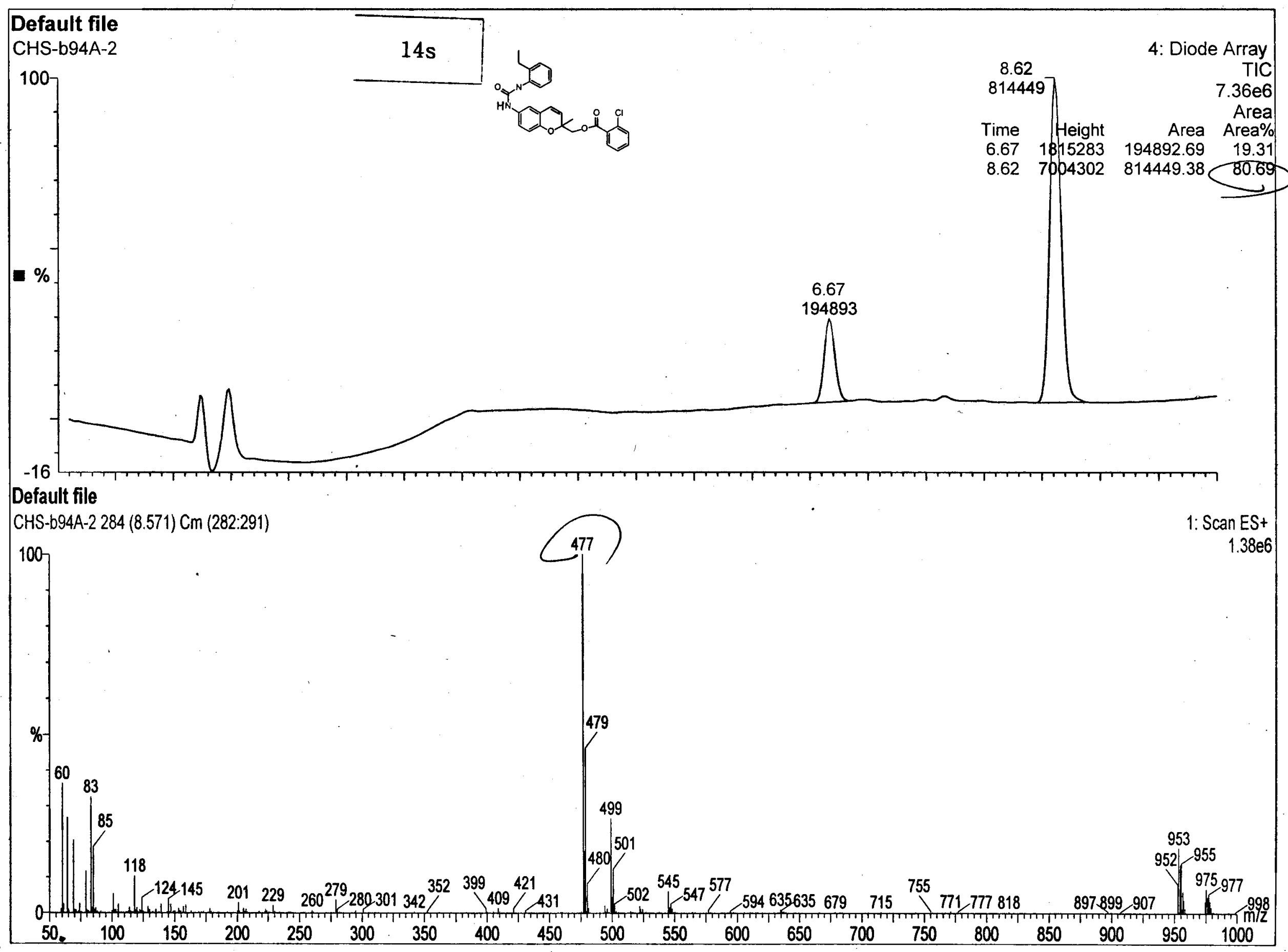




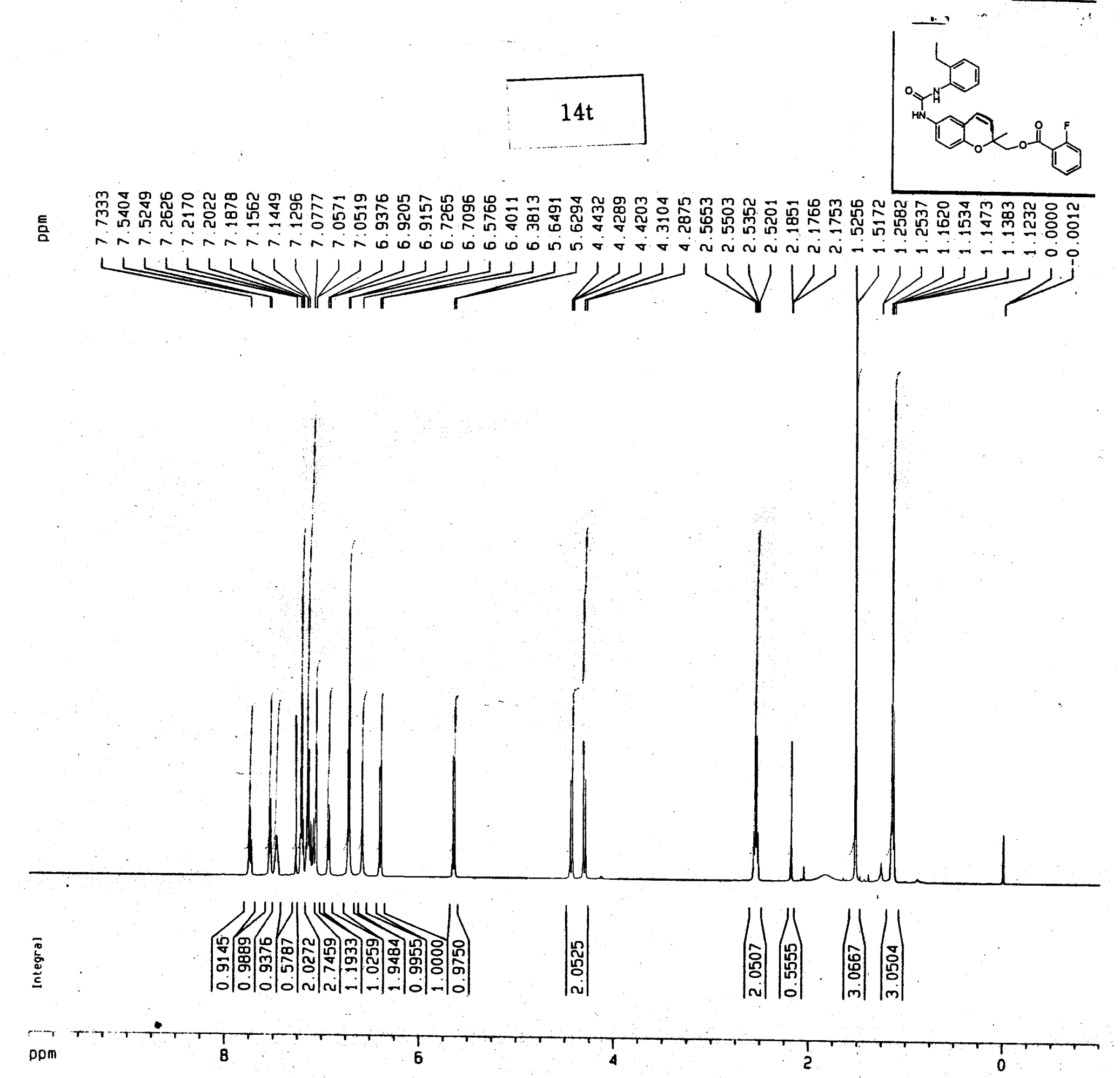

Current Data Parameters

NAME

PROCNO

F2 - ACquisition Parameters

Date_ 20041109

Time $\quad 23.58$

INSTAUM spect

PROBHD $5 \mathrm{~mm}$ DUL $13 \mathrm{C}-1$

PULPROG

TD $\quad 65536$

SOLVENT COC13

NS

DS

SWH $\quad 7507 \quad 507$ HZ

FIDRES $\quad 0.114555 \mathrm{~Hz}$

$\triangle 0 \quad 4.3648143 \mathrm{sec}$

RW 114

$\begin{array}{lr}\text { DH } & 66.600 \text { usec } \\ \text { DE } & 6.00 \text { usec }\end{array}$

TE $0.0 \mathrm{~K}$

$\quad 1.00000000 \mathrm{sec}$

MCREST $\quad 0.00000000 \mathrm{sec}$

MCWRK $\quad 0.01500000 \mathrm{sec}$

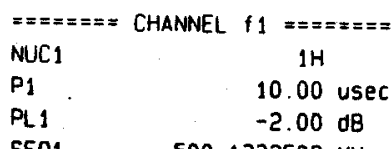

SF01 $500-2.00 \mathrm{~dB}$

F2 - Processing parameters

SI 32768

SF $\quad 500.1300130 \mathrm{MHZ}$

WDW EM

SSB

LB

PC

$E M$
0
$0.30 \mathrm{~Hz}$
0
1.00

10 MMA plot parameters

CX

Cr. $\quad 12.00 \mathrm{~cm}$

FIP $\quad 10.000 \mathrm{ppm}$

F1 $\quad 5001.30 \mathrm{~Hz}$

F2P $\quad-1.000 \mathrm{DDm}$

F2 $\quad-500.13 \mathrm{Mz}$

PPMCM $\quad 0.55000 \mathrm{pom} / \mathrm{cm}$

$\mathrm{HZCM} \quad 275.07150 \mathrm{~Hz} / \mathrm{cm}$ 


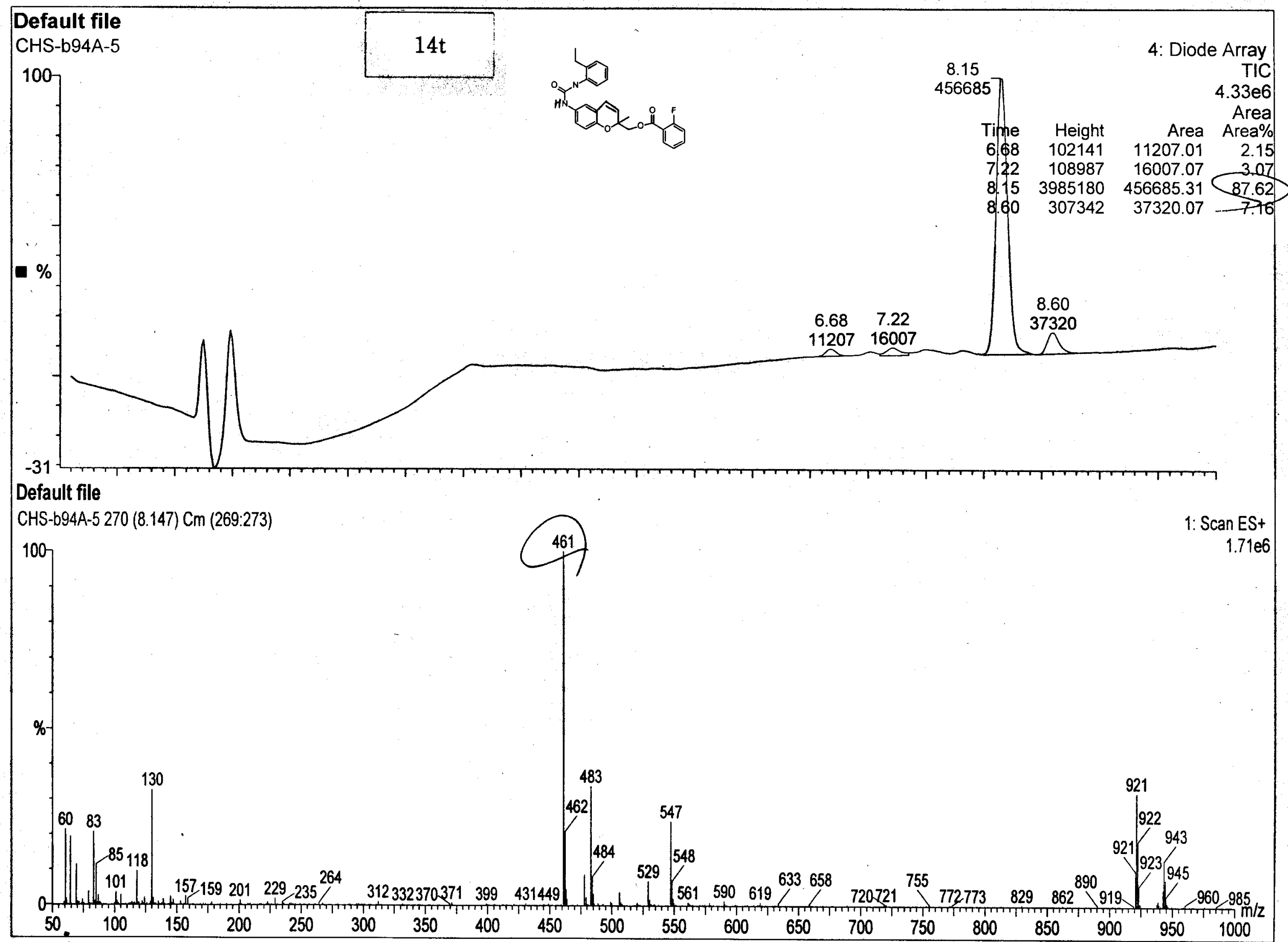



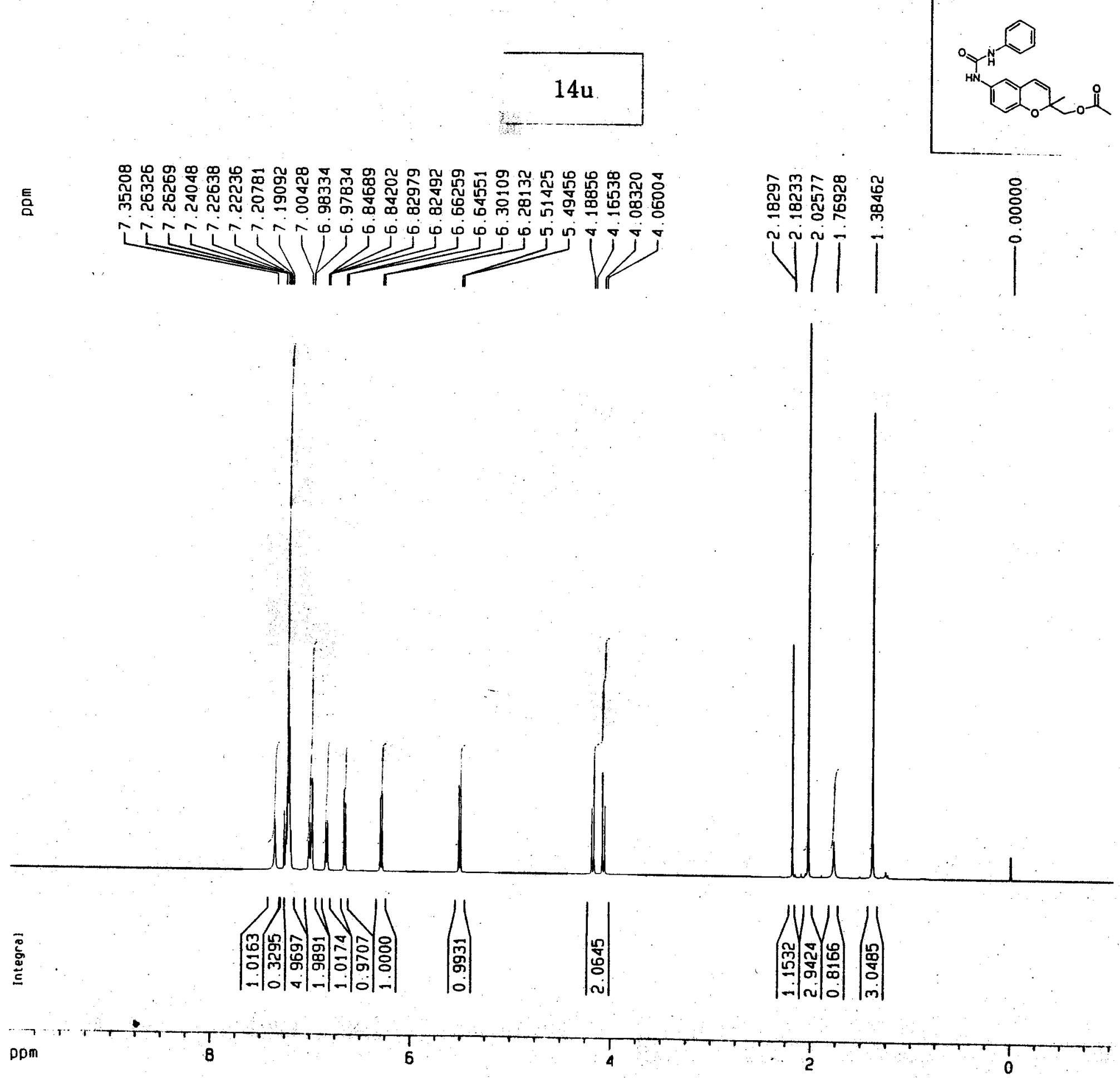


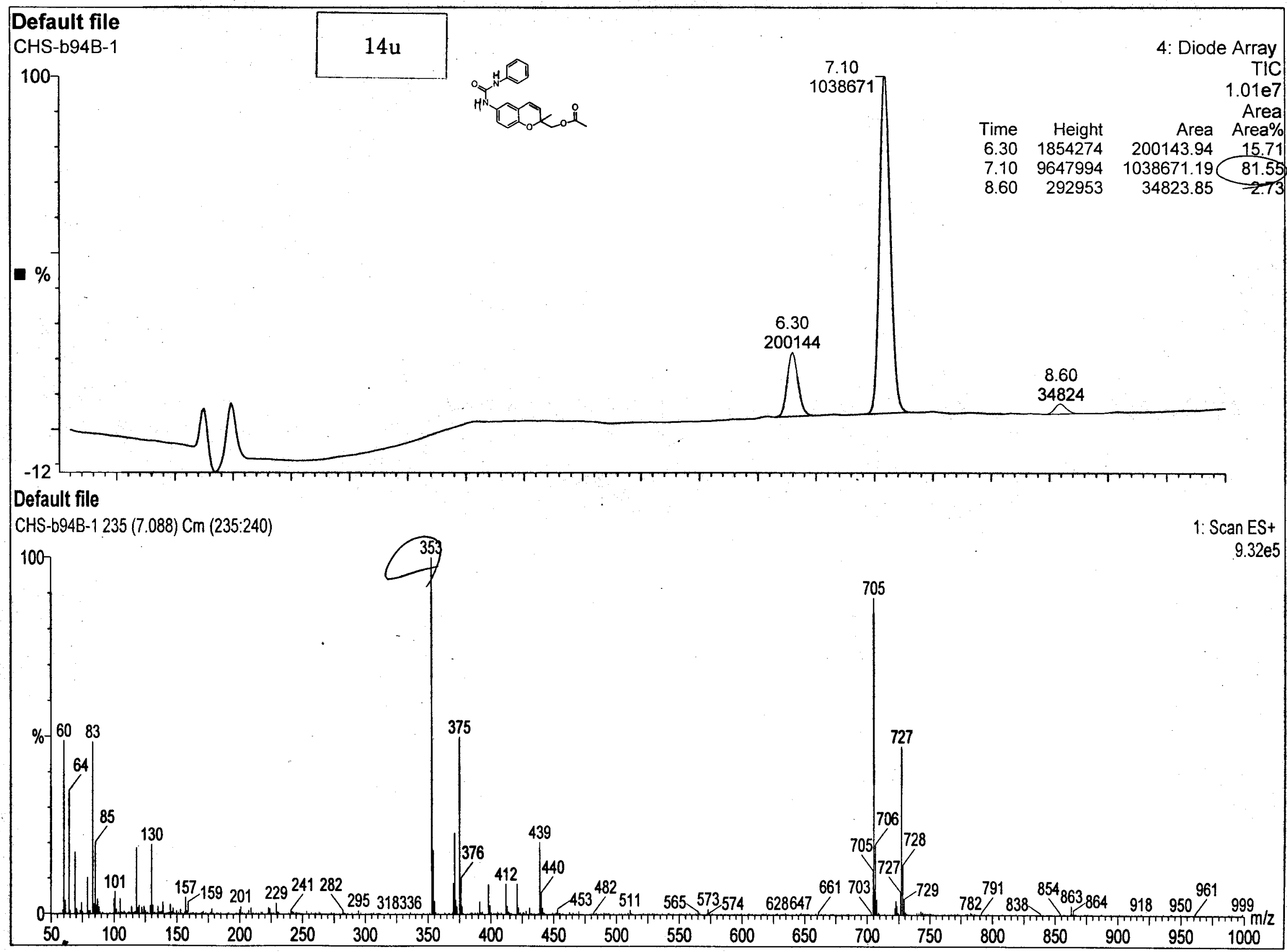

